\title{
Laser amplification in excited dielectrics
}

\section{Dissertation}

zur Erlangung des akademischen Grades eines Doktors der Naturwissenschaften (Dr. rer. nat.) im Fachbereich Mathematik und Naturwissenschaften der

\section{Universität Kassel}

\author{
vorgelegt von: \\ THOMAS TORSTEN WINKLER \\ aus Berlin
}

Kassel, Januar 2018 
Dissertation eingereicht am 21. November 2017 im Fachbereich 10 Mathematik und Naturwissenschaften der Universität Kassel.

1. Gutachter und Prüfer: Prof. Dr. Thomas Baumert

2. Gutachter und Prüfer: Prof. Dr. Peter Balling

Prüfer:

Prof. Dr. Thomas Giesen

Prüfer:

Prof. Dr. Martin Garcia

Tag der Disputation: 10. Januar 2018 
Progress is made by trial and failure; the failures are generally a hundred times more numerous than the successes; yet they are usually left unchronicled.

Sir William Ramsay, 1852 to 1916. 



\section{Danksagung / Anerkendelser}

Hätte mir jemand während meiner Oberstufenzeit in der Schule gesagt, dass ich mal in Physik promoviere, hätte ich wahrscheinlich nur mit dem Kopf geschüttelt. Denn zu der Zeit konnte ich mir dies nun wirklich nicht vorstellen. Doch dies änderte sich zum ersten Mal so richtig, als ich mit meiner Bachelorarbeit in der Arbeitsgruppe von Prof. Baumert begonnen habe. Hier kam ich mit dem Thema der Laser-angeregten Dielektrika in Kontakt, das mich dann insgesamt über fast 7 Jahre an der Uni Kassel während der Bachelor- und Masterarbeit, sowie der Promotion beschäftigt und zu vielen schlaflosen Nächten geführt hat. Denn ich hatte etwas gefunden, das meine Neugier derart geweckt hat, dass ich die Zeit, die ich mit Nachdenken, Experimentieren oder Simulieren verbracht habe, nicht nur Pflicht war, sondern es sehr gerne tat und mir viel Spaß machte. Dass ich das tun konnte, was mich erfüllt und ich auch noch dafür bezahlt wurde, für dieses Privileg bin ich sehr dankbar.

Lieber Herr Baumert, durch Sie habe ich nicht nur meine Anreden in Emails zu "Lieber Herr/Liebe Frau..." geändert, sondern durch Sie auch den bestmöglichen Start in die Zeit nach der Promotion erhalten. Sie haben es mir ermöglicht meine Abschlussarbeiten und meine Promotion in ihrer Arbeitsgruppe anzufertigen, aber haben mich auch in all meinen anderen Bestrebungen stets unterstützt. Seien es Tagungsteilnahmen, durch die ich viele wichtige Erfahrungen machen und Kontakte knüpfen konnte; Stipendien, Preisausschreibungen oder aber auch meinen Wunsch für eine Zeit nach Aarhus zu gehen und dort Ergebnisse zu diskutieren. Sie hatten stets ein offenes Ohr und viele Lösungsvorschläge parat. Auch wenn mich das Projekt zweitweise fast zum Verzweifeln gebracht hat, so hat es bei mir am Ende zu großer Eigenständigkeit und Selbstvertrauen in die eigenen Fähigkeiten geführt. Dies wird mir auf meinem weiteren Wege mit großer Sicherheit helfen. Vielen Dank für Ihre stete Unterstützung!

Dear Peter, I thank you for our great cooperation, your support and help throughout the LADIE project. Without you, I probably would have been very desperate while thinking and spinning my head about the simulations and calculations of the process. In addition, you always asked the right questions, even if it meant that I have to re-think and to start the simulations all over again. At the end, you truly helped to develop the proposed process of LADIE which can now be reproduced by the simulations, and I still cannot really believe up to this day. I hope that one of the many proposals will be granted, so that we can continue our common work in Aarhus! Thank you very much Peter!

Ich danke Prof. Thomas Giesen und Prof. Martin Garcia für Ihre Bereitschaft sich als Prüfer für meine Arbeit bereitzuerklären.

Das Experiment, an dem Cristian Sarpe mit mir während meiner Bachelorarbeit gearbeitet hat, war ein essentieller Teil, auf den ich in den folgenden Jahren in der Masterarbeit und Promotion aufbauen konnte. Auch während dieser Zeiten stand Cristian stets mit Rat und Tat zur 
Seite und hat viele wichtige Komponenten gebaut, die zu der Entdeckung der Lichtverstärkung in angeregten Dielektrika geführt hat! Ich habe sehr, sehr viel von dir gelernt. Vielen, vielen Dank Cristian!

A very special thank goes to Lasse Haahr-Lillevang, who was a visiting PhD Student for three months in the beginning of 2015, shortly after I started my PhD. Honestly, at first I didn't really knew what I should think about you joining me for my experiments. You were also modeling and measuring optical properties of excited dielectrics, so it sounded like a big competition. However, it turned out to be the best thing that could happen to me because we make a very effective and great team, that complements each other perfectly! We often discussed about the little things for hours but, we just had the right scepticism that pushed us to do our best and made us look for many details that might just would have get lost otherwise. Often, we realized much later, that we actually agreed in the first place. Within a few months, we managed to set up the spectral interference experiments on solids that would otherwise probably have taken a much longer time doing it on my own. In the same way it was nice to work with you in Aarhus, when I was visiting in 2016, where we discussed our experimental results, which did not really want to fit to what was expected. But in the end, we found the answers we were looking for! I am very grateful to have worked with you, but there is one thing I regret and this is that we did not took more opportunities for an $\varnothing 1$ efter arbejde while you were in Kassel! Thank you also for cross reading my thesis. I will see you in Aarhus!

Dear Sean, I thank you very much for taking your time to cross read the thesis prior to publication!

Ein besonderes Dankeschön geht an Nadine Götte, Bastian Zielinski und Nikolai Jelzow, meine (zeitweisen) MitstreiterInnen im Materialbearbeitungs-Team. Nadine, zusammen mit dir zu versuchen die tiefen Ablationsstrukturen in Quarzglas zu verstehen, war eine tolle Erfahrung. Zwar lief unsere Kommunikation zunächst nicht immer ohne Missverständnisse ab, aber wir fanden unseren Weg und schafften es das Geheimnis zu lösen und so auch meine Simulationen stets zu verbessern. Bastian, dir haben ich und Lasse viel zu verdanken, denn du hattest immer ein offenes Ohr für uns, wenn wir mal wieder Probleme mit der LabView-Programmierung hatten und hast uns geholfen diese zu lösen. Nie hast du auch nur einen Moment gezögert, wenn wir deine Hilfe brauchten! Die langen Diskussionen mit euch Beiden, Nadine und Bastian, waren oft sehr fruchtvoll und spaßig. Umso schöner war es, dass wir alle auch viel über Themen sprachen, die rein gar nichts mit der Arbeit zu tun hatten. Ich danke euch für die schöne Freundschaft und hoffe, dass sie erhalten bleibt, selbst wenn uns ein paar Kilometer trennen!

Liebe Petra, liebe Ute, ich glaube es bedürfe sehr vieler Seiten, würde man versuchen aufzuschreiben, wie wertvoll und wichtig ihr für die Arbeitsgruppe und vor allem uns Doktoranden seid. Ihr kümmert euch um die vielen Dinge, die vielen Aufgaben, die gemacht und erledigt werden, oft ohne dass man etwas davon mitbekommt. Damit macht ihr uns das Leben und unsere Arbeit so viel einfacher, obwohl dies im Alltag manchmal leider zu wenig Wertschätzung erfährt. Eure Türen stehen uns stets offen und so habt ihr immer ein offenes Ohr für uns, egal ob wir mit freudigen Nachrichten oder mit unseren Sorgen zu euch kommen, ihr seid immer für uns da. Und so hätte ich in meinen Jahren in der Arbeitsgruppe mit Sicherheit viel weniger Freude gehabt und so manche Frustphase weniger gut und schnell überwunden. Vielen lieben Dank euch Beiden!

Lieber Philip, dich als mein Büro-Nachbarn zu haben war und ist super. Ich danke dir für all unsere vielen langen und oft auch sehr lustigen Gespräche über die angenehmen Dinge des Lebens, wie 
gutes Essen und Trinken. Ich freue mich schon aufs nächste Grillen zum Mittag! Ich hoffe du findest deinen Weg, der dir viel Freude und Zufriedenheit bringt!

Lieber Arne, dir Danke ich für die vielen gemeinsamen wissenschaftlichen Diskussionen und Ratschläge, genauso wie dem Rest der Arbeitsgruppe für eine tolle, freundschaftliche, Atmosphäre die zu vielen lustigen und spannen Diskussionen geführt hat. Für euch habe ich sehr gerne hin und wieder gekocht oder Eis gemacht und würde es jeder Zeit wieder tun. Eigentlich könnte man denken, dass hier die Danksagung zu Ende ist. Aber, es gehört für mich mehr dazu als ein tolles Promotionsthema, eine tolle Arbeitsatmosphäre um so viel Spaß und auch Erfolg zu haben. Denn es hat sich gezeigt, dass in einer Promotion und auch schon während der Masterarbeit die Phasen der Hochfreude und der Verzweiflung oft sehr nah beieinanderliegen und dann ist es gut, wenn man liebevolle Menschen um sich hat, die einen verstehen und unterstützen. Hier danke ich besonders dir Jutta, da du dir sehr, sehr oft meine überschwänglich erzählten Ideen anhören musstest, wenn ich mal wieder mitten am Abend oder am Wochenende Einfälle zum LADIE Prozess hatte, selbst wenn es völlig aus dem Zusammenhang gerissen war. Genauso warst du auch immer in den Zeiten für mich da, wenn es mal wieder eine Phase der Verzweiflung gab und hast mir geholfen sie zu überwinden. Vielen Dank!

Auch all den anderen Menschen die mich über die Jahre begleitet und so Kassel für mich zu einer zweiten Heimat gemacht haben, vielen Dank.

$\mathrm{Zu}$ allerletzt möchte ich den Menschen danken, die den allergrößten Anteil an dem erfolgreichen Abschließen meiner Promotion haben und vor allem daran, dass es überhaupt so weit gekommen ist: Meinen Eltern, Marion und Torsten, sowie Oma Erika. In all den Jahren seit dem Beginn meines Studiums habt ihr mich immer unterstützt. Habt nie meine Entscheidungen angezweifelt, sei es nun in Kassel zu studieren, das Maschinenbaustudium abzubrechen und mit Physik anzufangen, noch nach dem Master zu promovieren anstatt "richtig" Arbeiten zu gehen. Ihr habt immer an mich, meine Entscheidungen und meinen Weg geglaubt und mich auch finanziell stark unterstützt. So habt ihr geholfen, einen meiner großen Träume während meines Master-Studiums zu verwirklichen, und zwar für ein Semester in den USA zu studieren. Ohne euch und die Werte, die ihr mir auf den Weg mitgegeben habt, wäre es nie dazu gekommen, dass ich promoviere. Vielen Dank für Alles, was ihr für mich getan habt! 



\section{List of publications}

Parts of this work were published in the following publications, listed in the reversed order of appearance:

- Thomas Winkler, Thomas Baumert, Arne Senftleben, Laser-Verstärkung in angeregten Dielektrika. Physik in unserer Zeit, Editor: Thomas Bührke and Roland Wengenmayr, Online ISSN 1521-3943, Volume 49, Issue 1, January 2018, Pages 9-10

- Thomas Winkler, Lasse Haahr-Lillevang, Cristian Sarpe, Bastian Zielinski, Nadine Götte, Arne Senftleben, Peter Balling and Thomas Baumert, Laser amplification in excited dielectrics. Nature Physics 14, $74-79$ (2018)

- Sebastien Courvoisier, Nadine Götte, Bastian Zielinski, Thomas Winkler, Cristian Sarpe, Arne Senftleben, Luigi Bonacina, Jean-Pierre Wolf, Thomas Baumert, Temporal Airy Pulses Control Cell Poration. APL Photonics, 2016, 1, 046102 (7pp)

- Thomas Winkler, Cristian Sarpe, Lasse Haahr-Lillevang, Nikolai Jelzow, Nadine Götte, Bastian Zielinski, Peter Balling, Arne Senftleben and Thomas Baumert, Probing spatial properties of electronic excitation in water after interaction with temporally shaped femtosecond laser pulses: experiments and simulations. Applied Surface Science, 2016, 374, 235-242

- Nadine Götte, Thomas Winkler, Tamara Meinl, Thomas Kusserow, Bastian Zielinski, Cristian Sarpe, Arne Senftleben, Hartmut Hillmer, Thomas Baumert, Temporal Airy pulses for controlled high aspect ratio nanomachining of dielectrics. Optica, 2016, 3(4), 389-395

- Nadine Götte, Thomas Kusserow, Thomas Winkler, Cristian Sarpe, Lars Englert, Dirk Otto, Tamara Meinl, Yousuf Khan, Bastian Zielinski, Arne Senftleben, Matthias Wollenhaupt, Hartmut Hillmer, Thomas Baumert, Temporally shaped femtosecond laser pulses for creation of functional sub-100 $\mathrm{nm}$ structures in dielectrics. In: Optically Induced Nanostructures, Editors: K. König, A. Ostendorf, De Gruyter, 2015, Chapter 3, 47-72 



\section{Contents}

\begin{tabular}{ll}
\hline Acknowledgments & 1
\end{tabular}

\begin{tabular}{ll}
\hline List of publications & 5
\end{tabular}

\begin{tabular}{lr}
\hline Table of contents & $\mathbf{9}$
\end{tabular}

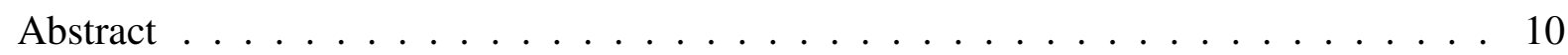

Kurzzusammenfassung . . . . . . . . . . . . . . . . . . 10

\begin{tabular}{ll}
\hline Introduction & 13
\end{tabular}

\begin{tabular}{lll}
\hline l. Basics & 15
\end{tabular}

$\begin{array}{ll}\text { 1. Description of Gaussian laser pulses } & 17\end{array}$

1.1. Spatial and temporal properties . . . . . . . . . . . . . . . . . . . . . . . . . . . . 17

1.2. Temporally shaped laser pulses . . . . . . . . . . . . . . . . . . . . . . . . . . 19

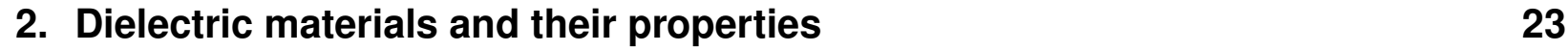

3. Excitation mechanisms 25

3.1. Strong-field excitation . . . . . . . . . . . . . . . . 25

3.1.1. Ponderomotive energy and band gap increase . . . . . . . . . . . 26

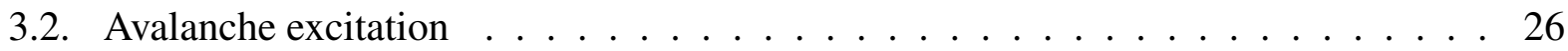

3.3. Intraband-thermalization . . . . . . . . . . . . . . . 27

3.4. Relaxation processes . . . . . . . . . . . . . . . 28

4. Optical properties of laser-excited dielectrics 31

4.1. Drude model $\ldots \ldots \ldots \ldots \ldots \ldots \ldots \ldots$

4.2. The Clausius-Mosotti correction . . . . . . . . . . . . . . . . . . . . . . . . . . . . . . . . 34

4.3. Lorentz-Drude Model . . . . . . . . . . . . . . . . . . . . . . . . . . . . . 34

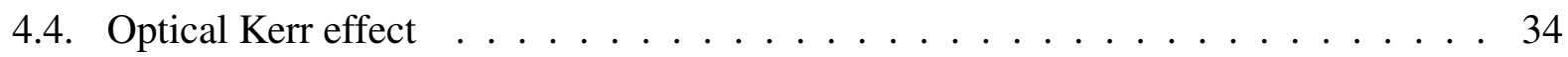

4.5. Propagation effectcs . . . . . . . . . . . . . . . . . . 35

$\begin{array}{ll}\text { 5. Modeling laser excitation } & 37\end{array}$

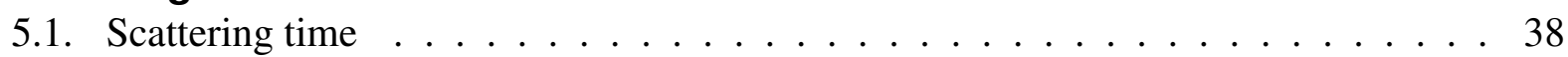

5.2. Multiple-Rate-Equation model . . . . . . . . . . . . . . . . . . . . 41

5.3. Modelling laser pulse propagation . . . . . . . . . . . . . . . . 41

5.3.1. Exemplary calculations . . . . . . . . . . . . . . . . . 44 
6. Modeling intraband thermalization in dielectrics 49

6.1 . Population inversion in excited dielectrics $\ldots \ldots \ldots \ldots 2$

7. Experimental techniques and the extraction of physical quantities 55

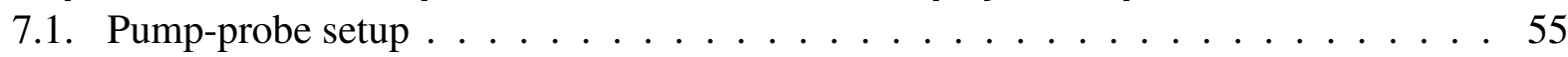

7.2. Common-path spectral interferometry setup . . . . . . . . . . . . . . . 57

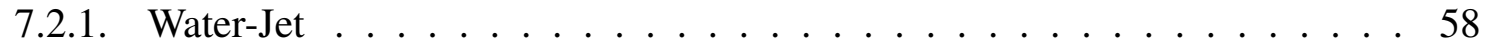

7.3. Extraction of physical quantities $\ldots \ldots \ldots \ldots \ldots$

7.3.1. Transmission . . . . . . . . . . . . . . . . 61

Transmission in pump-probe $\ldots \ldots \ldots \ldots \ldots$

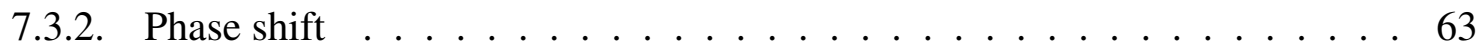

II. Laser amplification in excited dielectrics 65

8. Introduction and outline 67

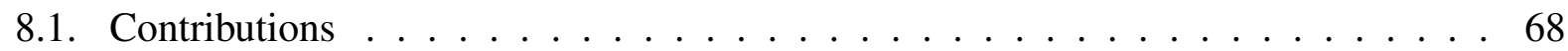

8.1.1. Experimental contributions . . . . . . . . . . . . . . 68

8.1.2. Theoretical contributions . . . . . . . . . . . . . . . . . . . . . 69

9. The experiment and experimental results 71

9.1. Temporal Dynamics of laser excited sapphire and fused silica . . . . . . . . . 73

9.1.1. Global and local dynamics in sapphire . . . . . . . . . . . . . . . . 73

Long delay scan in sapphire $\ldots \ldots \ldots \ldots$. . . . . . . . . . . . 75

9.2. Pump pulse fluences dependence in sapphire . . . . . . . . . . . . . . . . 76

9.3. Spectral properties of excitation in sapphire $\ldots \ldots \ldots \ldots \ldots$

9.4. Probe pulse fluence dependence in sapphire $\ldots \ldots \ldots \ldots$

9.4.1. Global behavior . . . . . . . . . . . . . . . . . . . . 80

9.4.2. Local behavior . . . . . . . . . . . . . . . . . . . 80

9.5. Comparison of global and local dynamics between sapphire and fused silica . . . . 81

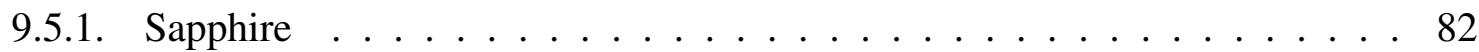

9.5.2. Fused silica . . . . . . . . . . . . . . . . . . . 82

9.5.3. Pump pulse fluence dependence in fused silica $\ldots \ldots \ldots$. . . . . 84

10.Proposed amplification mechanism of LADIE and simulations 85

10.1. Simulations of LADIE and comparison to the experiment $\ldots \ldots \ldots$. . . . . 88

10.2. Optimizing the amplification by using temporally shaped pump pulses $\ldots \ldots$. . . 90

$\begin{array}{ll}\text { 11.Short summary and outlook } & 93\end{array}$

III. Spatial properties of laser excitation in water 97

$\begin{array}{ll}\text { 12.Introduction and outline } & 99\end{array}$

$\begin{array}{ll}\text { 13. Experimental results } & 101\end{array}$

13.1. Bandwidth limited laser pulses . . . . . . . . . . . . . . . . . . . . 101

13.2. Temporal Airy pulses . . . . . . . . . . . . . . . . . . . . . . . . 104 
13.3. Fluence dependence of the phase shift: Center vs Radial dependency . . . . . . . . 104

14. Comparison of measurement to simulations 107

14.1. Radial dependent phase shift . . . . . . . . . . . . . . . 107

\begin{tabular}{ll}
\hline 15. Short summary & 113
\end{tabular}

\begin{tabular}{lr}
\hline IV. Summary & 114
\end{tabular}

\begin{tabular}{ll}
\hline V. Appendix & 117
\end{tabular}

\begin{tabular}{ll}
\hline 16. Additional data to part II & 118
\end{tabular}

16.1. Sapphire . . . . . . . . . . . . . . . . . . . 118

16.1.1. CCD Images of the delay scan . . . . . . . . . . . . . . . . . . . . . . . . . . . . . . . . . .

16.1.2. Delay scan using a low pump pulse fluence . . . . . . . . . . . . . . . 118

16.1.3. Delay scan at a moderate pump pulse fluence . . . . . . . . . . . . . . 122

16.1.4. Pump pulse fluence dependence at different probe pulse fluences . . . . . . 122

16.1.5. Influence of pump pulse polarization . . . . . . . . . . . . . . . . 124

16.1.6. Spectral-interference measurement under amplification conditions . . . . . 124

16.2. Fused silica $\ldots \ldots \ldots \ldots \ldots \ldots$

16.2.1. Delay scan under LADIE conditions . . . . . . . . . . . . . . . . . . . . . . . . . . . . . . .

16.2.2. Delay scans at different pump pulse fluences . . . . . . . . . . . . . 127

Phase shift . . . . . . . . . . . . . . . . . . 127

Transmission . . . . . . . . . . . . . . . . . . . . 129

16.2.3. Intensity scan . . . . . . . . . . . . . . . . . . . . . . . . . . . . . . . . . . . . . . . .

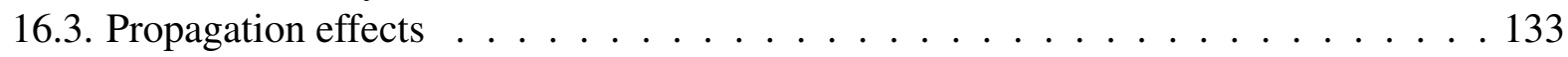

16.3.1. Influence of beam profiles . . . . . . . . . . . . . . 135

17. Location of used measurement and evaluation software and experimental data $\quad 137$

17.1. Overview of measurements for part II . . . . . . . . . . . . . . . 137

17.2. Overview of measurements for part III . . . . . . . . . . . . . . . . . 139

\begin{tabular}{ll}
\hline 18. Overview of the most important symbols & 140
\end{tabular}

\begin{tabular}{ll}
\hline Symbols and quantities & 140
\end{tabular}

19. Quantities for the calculation of laser excitation of sapphire 142

20. Quantities for the calculation of laser excitation of water $\quad 144$

\begin{tabular}{ll}
\hline Bibliography & 146
\end{tabular} 


\section{Abstract}

Today, laser processing of dielectric materials with ultrashort, femtosecond laser pulses finds a great variety of applications. While ablation, cutting or welding of different types of glasses are widespread and popular examples, nanostructuring of transparent crystals or optoporating cells is part of more recent scientific studies. Although the material systems seem to be very different, their interaction with visible and infrared wavelengths is very similar: the light is simply transmitted. This changes drastically when they are exposed to ultrashort and highly intense laser pulses. Different interaction mechanisms lead to the appearance of a transient metal-like state. Thus, pump-probe experiments are the method of choice to study the optical excitation of dielectric materials. In strong contrast to the metallic characteristics of excited dielectrics, this study provides the first observation of the amplification of a probe laser in optically excited sapphire and fused silica glasses. Not only is the observed amplification of coherent nature, it is also dependent on the incident probe pulse intensity and thus of a nonlinear order, a curiosity in itself. From the experimentally obtained energetic and temporal dependencies, a consistent three-step model was developed and is supported by simulations. Besides, the influence of temporal pulse shaping on spatial properties of the laser excitation in water is demonstrated experimentally and via simulations. It is shown that temporally asymmetric shaped laser pulses lead to a spatial distribution of laser excitation that is reduced in area but highly extended in depth. The results are put into the context of post-mortem experiments.

\section{Kurzzusammenfassung}

Die Lasermaterialbearbeitung dielektrischer Materialien mittels ultrakurzer Femtosekundenlaserpulse findet in der heutigen Zeit vielfältige Anwendungen. Während das Abtragen, Trennen oder Fügen diverser Gläser sehr naheliegende und weit verbreitete Anwendungen sind, ist die Nanostrukturierung von Kristallen oder die Optoporation von Zellen Teil aktueller Studien. Doch egal wie unterschiedlich die Materialsysteme erscheinen, ihre Wechselwirkung mit sichtbarem und infrarotem Licht ist identisch, es tritt ohne Absorption hindurch. Dies ändert sich jedoch abrupt, wenn ultrakurze Laserpulse im Femtosekunden-Bereich verwendet werden: Durch die sehr hohen Intensitäten, die diese kurzen Laserpulse haben, können verschiedene Wechselwirkungsmechanismen einen Übergang vom transparenten in einen transienten metallischen Zustand bewirken, was eine starke Absorption und Reflexion zur Folge hat. Dementsprechend haben sich Anrege-Abfrage Experimente als eine sehr gute Methode bewährt, um die optische Anregung dielektrischer Materialien zu untersuchen. In teils starkem Kontrast zu bisher beobachteten Effekten stehen die in dieser Arbeit präsentierten Untersuchungen an kristallinem Saphir und Quarzglas: Zum ersten Mal konnte gezeigt werden, dass unter bestimmten experimentellen Bedingungen ein zeitlich verzögerter Abfragepulse in einem optisch stark angeregten Dielektrikum verstärkt wird. Dabei ist diese Verstärkung nicht nur, ähnlich wie in einem klassischen Laser, kohärent, sondern hängt auch von der eingehenden Intensität des verstärkten Laserpulses ab. Die Verstärkung ist nichtlinear und stellt somit eine selten beobachtete Kuriosität dar. Unterstützt werden die Messungen, die die zeitlichen und energetischen Abhängigkeiten aufzeigen, durch einen Erklärungsansatz mittels eines einen mehrstufigen Prozess und dessen Simulation. Zudem wird der Einfluss zeitlich asymmetrisch geformter Femtosekunden Laserpulse auf die räumliche Verteilung der Anregung in Wasser mittels Experiment und Simulation untersucht. Es zeigt sich, dass zeitlich asymmetrisch 
geformte Laserpulse zu einer räumlichen Anregungsverteilung führen, die im Vergleich zu bandbreitebegrenzten Laserpulsen eine um die Hälfte reduzierten Fläche hat, dafür aber umso größere Tiefe erreicht. Diese Ergebnisse werden im Kontext aktueller post-mortem Experimente diskutiert. 



\section{Introduction}

$\mathrm{F}^{\mathrm{a}}$

OCUSING a near infra-red femtosecond laser pulse on or inside transparent materials such as glasses or tissue leads to a well-defined deposition of energy by promoting a large density of carriers to the conduction band by strong-field excitation processes. As this energy stays spatially confined on ultrashort time scales, these laser pulses have become the instrument of choice for dielectric structuring on a micrometre or even nanometre scale [1] as well as for applications in nanosurgery [2]. Depending on the incident intensity, a large range of material modifications can be realized: While optical Kerr effect, self [3]- and cross-phase [4] modulation or field-induced currents [5] are examples of reversible processes, higher intensities can cause permanent refractiveindex changes [6,7] ablation of the material or even the creation of new material phases [8]. It is the transient conduction-band-electron density that plays the fundamental role in determining the optical properties and time-resolved pump-probe experiments are an excellent tool for studying its dynamics, demonstrated in a manifold of experiments. Nearly all of them show that absorption and reflection strongly increase after excitation due to the build-up of a transient electron plasma. This gives the material transient metallic properties that eventually prevent transmission through the dielectric material. The above-mentioned processes and properties are discussed and described in the first part (I) of the thesis, together with the experimental setup and how the data was evaluated. When physical mechanisms are introduced and discussed, they are often accompanied by calculations to visualize certain effects and dynamics. All calculations and visualizations are done for sapphire, a material with many known material parameters, given in table 19.1 in the appendix.

The most important goal of this thesis was to extend the existing experimental spectral interference setup on liquid [9] to solid samples, allowing an in-situ characterization of the strength of excitation in solid dielectrics like fused silica and sapphire, as the understanding of the interaction with ultrashort and temporally shaped femtosecond laser pulses can be much easier resolved than compared to post-mortem experiments. The setup of the experiment was realized with the help of Lasse Haahr-Lillevang who was a visiting PhD student from the University of Aarhus for three months in early 2015 (see description in chapter 7). The first experiments on sapphire and fused silica demonstrated immediately the high temporal and spatial resolution in the investigation of optical properties of laser-excited dielectrics in a (reference-) pump-probe experiment. But, something did not seem quite right: We observed that the $400 \mathrm{~nm}$ probe pulse is coherently amplified inside the sapphire sample on a scale of a few micrometers for certain excitation conditions induced by the $800 \mathrm{~nm}$ pump pulse. To confirm these observation, the experiment was changed into a pure-pump probe configuration and was supported by simulations in which Lasse Haahr-Lillevang was again involved. They strongly support the proposed two-photon stimulated emission process and reproduce all main experimental observations. Thus, this thesis demonstrates the first straightforward experimental proof of coherent laser amplification in excited dielectrics (LADIE). The results were published in Nature Physics [10] and are described in part III]

The typical characteristic behavior of excited dielectrics, increasing absorption and change in refractive index, is often used to quantify the level of excitation and allows to investigate a manifold 
of dependencies like the temporal dynamics, strength of excitation or even the impact of the temporal pulse shape. In this context, we found in our previous study [11] indications that the utilization of temporally asymmetrically shaped femtosecond laser pulses leads to higher excitation inside water. In extension, this study provides the first insight into the spatial properties of laser excitation of water using ultrashort, bandwidth-limited and third order dispersion shaped laser pulses. Not only is the pump-strength dependence measured spatially-resolved (see chapter 7), they are accompanied by simulations that allow understanding how such temporal pulse shapes indeed lead to an excitation reaching much deeper into the sample, while at the same time the area of excitation is reduced by nearly a factor of two. These results were published in Applied Surface Science [12] and are discussed in part III.

Similar results that link spatial properties of laser excitation to the temporal pulse shape were obtained in a recent study of ablating fused silica, performed by Nadine Götte [13, 14]: It was found that by utilizing the same pulse shapes as mentioned above, ablation structures with huge aspect ratios (e.g. $250 \mathrm{~nm}$ width to $10 \mu \mathrm{m}$ in depth) were created. This was the first demonstration of creating such structures with a single pulse, by pure temporal pulse shaping, without utilizing spatial beam shaping techniques [15] or filamentaion processes. Although much harder focusing conditions were used in comparison to the water experiments, the spatial characteristics of the ablation were much more overwhelming. To understand the differences between the observations on the two different dielectric materials, we adapted the simulation to fused silica. The main difference between water and fused silica in context of this thesis is, besides the state-of-matter at room temperature, the ultra fast trapping of electrons in exitonic states within $150 \mathrm{fs}$. These states are not linearly absorbing the near IR-wavelengths of our pump pulses, however as they are located within the band gap, they are more easily excited than the electrons from the valence band. By considering not only multi-photon excitation, but also, for the first time, avalanche excitation from these states we were able to simulate the ablation depth for a great variety of pulse shapes and focusing conditions in an overwhelming agreement with the experimental results. While the experiments were performed by Nadine Götte, I adapted my simulation model, originally written for water, and performed the calculations. The results were published in [13] and are discussed in great detail in the PhD-thesis of Nadine Götte [14]. 


\section{Part I. \\ Basics}





\section{Description of Gaussian laser pulses}

\subsection{Spatial and temporal properties}

To describe ultrashort laser pulses, we use the linearly polarized electric field $E$ at a fixed position in space as a function of time $t$. The real valued time-dependent electric field $E(t)$ consists of an envelope function $A(t)$ and the oscillating wave described by:

$$
E(t)=A(t) \cos \left[\Phi_{0}+\omega_{1} t\right]
$$

where $\omega_{1}$ is the angular frequency of the laser pulse. For our laser pulses with a central wavelength $\lambda_{l}$ of $800 \mathrm{~nm}$, we obtain via

$$
\omega_{1}=c \frac{2 \pi}{\lambda_{l}},
$$

an angular frequency of $\omega_{1}=2.34 \mathrm{rad} / \mathrm{fs}$ using the speed of light in vacuum $c$. This is equal to a period of $2.67 \mathrm{fs}$. $\Phi_{0}$ describes the phase between the envelope and the oscillating field.

The time dependent, oscillating laser intensity $I(t)_{o s c}$ is defined by [16]:

$$
I(t)_{o s c}=\varepsilon_{0} c n_{0}|E(t)|^{2}=\varepsilon_{0} c n_{0} A(t)^{2} \cos ^{2}\left[\Phi_{0}+\omega_{1} t\right],
$$

where $\varepsilon_{0}$ is the vacuum permittivity and $n_{0}$ the refractive index of the material. As the envelope $A(t)$ is only slowly varying with respect to the fast oscillating part, usually the cycle-averaged description $]^{1}$ is used [16]:

$$
I(t)=\frac{1}{2} \varepsilon_{0} c n_{0} A(t)^{2}=I_{0} \exp \left(-4 \ln 2\left(\frac{t}{\Delta t}\right)^{2}\right),
$$

where $I_{0}$ is the peak-intensity of the laser pulse and the last term the Gaussian intensity distribution in time with a FWHM pulse duration $\Delta t$. The pulse duration can be calculated from the measured spectrum of the laser pulse via the bandwidth-time product (see chapter 1) or measured as will be discussed later.

The peak intensity $I_{0}$ can be calculated from the experimentally determined pulse duration, pulse energy $E_{P}$ and $1 / e^{2}$ beam waist $w_{0}$ via [16]:

$$
I_{0}=\sqrt{\frac{4 \ln 2}{\pi}} \frac{2 E_{P}}{\pi w_{0}^{2} \Delta t} .
$$

It can be seen by comparing the cycle-averaged and the oscillating intensity, that the latter has a two times higher peak intensity. This could potentially have an impact, as many of the processes

\footnotetext{
${ }^{1}$ Cycle-average means that the oscillating intensity is integrated over one period.
} 
introduced and discussed in this work are depending on the strength of the electric field or laser intensity. All the descriptions for the different field driven effects introduced in this work were derived for the cycle-averaged laser pulses and are even valid down to the single-cycle regime, as discussed in Brabec and Krausz [17]. It will be noted when we use the description of the oscillating electric field.

The spatial intensity distribution of our Gaussian laser pulse is described by [16]:

$$
I(r)=I_{0} \exp -\left(2 \frac{r^{2}}{w_{0}^{2}}\right),
$$

where $I_{0}$ is the, already introduced, peak-intensity at the center $(r=0)$ of the pulse and $w_{0}$ is the spot size, defined as the radius at which the intensity is decreased to $1 / e^{2}$ of the peak value $I_{0}$.

Although it is usually not possible to directly measure the intensity of an ultrashort laser pulses, the temporally integrated transmission, the local fluence given by:

$$
F(r)=\int_{-\infty}^{\infty} I(t, r) \mathrm{d} t
$$

is a value that can be measured, e.g. by a calibrated CCD ("CCD": Charged coupled device) camera.

If the fluence is integrated over space:

$$
E_{P}=\int_{0}^{\infty} F(r) \mathrm{d} r
$$

we obtain the pulse energy, $E_{P}$, of the laser pulse, representing the spatially and temporally integrated intensity $I(t, r)$.

Using the above mentioned relations, the peak fluence $F_{\text {Peak }}$, the cycle-averaged peak intensity and pulse energy can be put into the following relation:

$$
F_{\text {Peak }}=\frac{1}{2} I_{0} \Delta t \sqrt{\frac{\pi}{\ln 2}}=\frac{2 E_{P}}{\pi w_{0}^{2}} .
$$

The energy of a laser pulse can be measured for example by a very fast photodiode or a slow photodiode/thermal powermeter measuring the average power of the laser. By taking into account the repetition rate of the laser $f$, the energy of a single pulse can be simply calculated from the average power $P$ by:

$$
E_{P}=\frac{P}{f}
$$

This is only valid if all pulses have the same pulse energy. Usually the pulse-to-pulse energy fluctuations are in the range of a few percent making it a valid measurement technique for the pulse energy. 


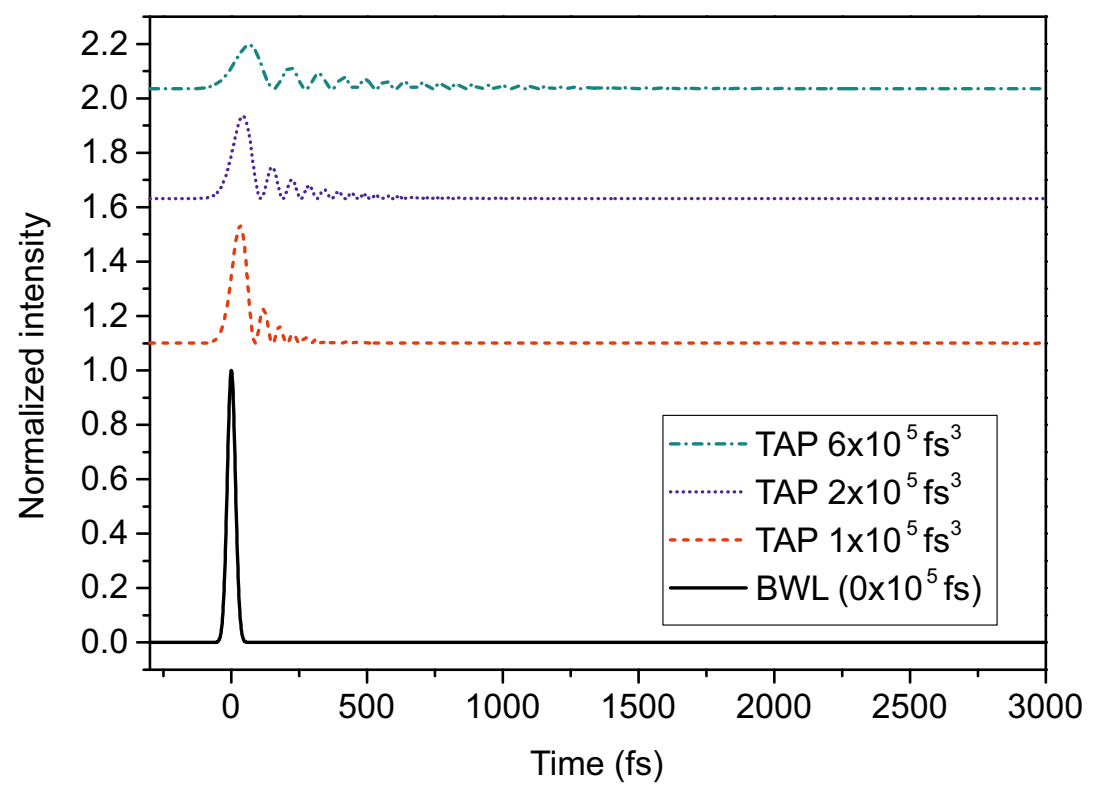

Figure 1.1.: Time dependent intensity profile (cycle-averaged) of a bandwidth limited (black line) and positively cubic phase shaped laser pulses with a $\phi_{3}$ of 100000,300000 and $600000 \mathrm{fs}^{3}$. The pulse shapes are of identical fluence and are shifted on the y-axis for a better visibility.

\subsection{Temporally shaped laser pulses}

In the presented experiments, we utilized various types of temporal pulse shapes. While a simple stretching of the laser pulse can be realized by the introduction of glass into the beam path, a free manipulation on a time scale of only tens of femtoseconds requires a different approach.

Ultrashort laser pulses consist of a very broad spectrum of tens of nanometers and therefore we make use of a spectral phase pulse shaper [18]. In such a device, the ultrashort laser pulse is split into its spectral components by a grating or a prism. Then, in a liquid crystal display (modulator) it is possible to delay each group of spectral components with respect to the others by introducing a spectral phase, $\phi(\omega)$. Temporal changes of the electric field are then defined by phase coefficients [19]:

$$
\phi_{i}(\omega)=\left.\frac{\partial \phi(\omega)}{\partial \omega^{i}}\right|_{\omega_{1}}
$$

where $\omega_{l}$ is the central frequency of the laser pulse. The phase coefficients are defined by a Taylor series of the spectral phase [18-[20]:

$\phi(\omega)=\sum_{0}^{\infty} \frac{\phi_{i}\left(\omega_{1}\right)}{i !}\left(\omega_{1}-\omega\right)^{2}=\phi_{0}\left(\omega_{1}\right)+\frac{1}{1 !} \phi_{1}\left(\omega_{1}\right)\left(\omega_{1}-\omega\right)+\frac{1}{2 !} \phi_{2}\left(\omega_{1}\right)\left(\omega_{1}-\omega\right)^{2}+\frac{1}{3 !} \phi_{3}\left(\omega_{1}\right)\left(\omega_{1}-\omega\right)^{3}+\ldots$

Afterwards, when the spatially separated frequency components of the pulse are recombined by a second grating or prism, the shifted spectral components will have a direct impact on the temporal shape of the pulse. Please note that only higher orders $(\geq 2)$ have an impact onto the temporal intensity distribution of the laser pulse [18, 19]. 


\section{Bandwidth-limited laser pulses (BWL)}

In case that the laser pulse has a constant $\phi_{i}=0$ for all $i$ or is described by a linear function, it is a bandwidth-limited- (BWL) or Fourier-transform limited laser pulse having a Gaussian intensity distribution (1.5). The FWHM pulse duration, $\Delta t$, is defined by the width of the frequency spectrum, $\Delta \nu$, via the Time-Bandwidth product for a Gaussian distribution in time and frequency [19, 20]:

$$
\Delta t \cdot \Delta \nu \approx 0.44
$$

For a frequency width of $1.467 \times 10^{13} \mathrm{~s}^{-1}$, we obtain a pulse duration of $30 \mathrm{fs}$.

\section{Group delayed dispersion shaped laser pulses (GDD)}

If a parabolic spectral phase function $\phi_{2} \neq 0$ is applied, we obtain a classical chirp of the laser pulse, characterized by a linear increase or decrease of the frequency as a function of time [19, 20]. A positive $\phi_{2}$ is called up-chirp, whereas for negative values it is a down-chirp. The case of an up-chirp is well known as the dispersion introduced by a piece of glass in the beam path. As the frequencies are shifted as a function of time, the pulse elongates and its FWHM pulse duration increases as a function of the chirp parameter [20]:

$$
\Delta t_{\mathrm{GDD}}=\sqrt{\Delta t^{2}+16 \cdot(\ln 2)^{2}\left(\frac{\phi_{2}}{\Delta t}\right)^{2}} .
$$

As the pulse duration increases, the intensity of the pulse will drastically decrease, as the pulse energy is conserved. In this study we only introduced group delay dispersion to compress the pulse in the interaction area, compensating all optical elements the pulse passed on its way.

\section{Third order dispersion shaped laser pulses (TOD)}

While the GDD introduced a symmetrical broadening of the laser pulse, a purely cubic phase function $\phi(\omega)=\frac{\phi_{3}}{3 !}\left(\omega_{1}-\omega\right)^{3}$ leads to a temporally asymmetric pules shape described by [20]:

$$
I(t)_{\mathrm{Mod}}=I_{0}\left[f\left(\frac{\Delta t}{\phi_{0}}\right) \exp \left(\frac{1}{2} \ln (2)\left(\frac{\left(\frac{2}{3} \mathbf{t}-t\right)}{t_{12}}\right)\right) \operatorname{AiryAi}\left[\frac{\mathbf{t}-t}{\delta \mathrm{t}}\right]\right]^{2},
$$

with:

$$
\begin{gathered}
f=\frac{\sqrt{\pi}}{2^{\frac{1}{6}} \sqrt{\ln [2]}}, \\
\phi_{0}=\phi_{3}^{\frac{1}{3}}, \\
\mathbf{t}=\left(\frac{\delta \mathrm{t}^{4}}{32 \phi_{3} \ln (2)^{2}}\right), \\
\delta \mathrm{t}=\left(\frac{\operatorname{Sign}\left[\phi_{3}\right]}{2^{\frac{1}{3}}}\right) \phi_{0},
\end{gathered}
$$


and $\operatorname{Sign}(x)$ as the Signum function. Furthermore $t_{12}$ is given by:

$$
t_{12}=\frac{2 \phi_{3} \ln [2]^{2}}{\delta \mathrm{t}^{2}}
$$

Examples for the temporal shapes of third order dispersion shaped pulses using a $\phi_{3}$ of 100000 , 200000 and $600000 \mathrm{fs}^{3}$ are shown in figure 1.1. For comparison, a BWL laser pulse with a FWHM of $\Delta t=30 \mathrm{fs}$ is shown. Please note that the BWL and the TOD shaped laser pulses have equal pulse energy, leading to a reduced maximum intensity of the strongest peaks of the TOD pulse train by about $1 / 6$ for a $\phi_{3}$ of $600000 \mathrm{fs}^{3}$. In addition, the peaks with the highest intensity are shifted to later times. With an increasing TAP parameter, the TOD shaped pulses consist of an increasing number of sub pulses and the temporal distance of the pulse train maxima increases. As TOD shaped laser pulses resembles the temporal equivalent of Airy pulses due to their similar shape, they are named "Temporal Airy pulses" (TAP) throughout this thesis. 



\section{Dielectric materials and their properties}

The linear interaction of light with material depends not only on the wavelength of the light but also on the properties of the material. Typically for the interaction of light in the visible or infrared regime with metals is a high reflectivity at the surface and a high absorption over the first tens of nanometers of the material. In dielectric materials, on the other hand, those wavelengths are readily transmitted, besides the small reflection at the interfaces.

The different behavior can be explained by the number of electrons in the conduction band. Since in metals, valence- and conduction band are very close together or are even overlapping, the population of electrons in the $\mathrm{CB}$ is very high [21]. In dielectrics, on the other hand, the conduction band is empty as thermal excitation is not possible due to the very large band gap that typically exceeds several electron volts $\left(E_{\mathrm{G}}>4 \mathrm{eV}\right)$. In comparison, the thermal energy of a free electron with three degrees of freedom at room temperature (21 degrees Celsius) is only $0.0375 \mathrm{eV}$. Examples for dielectric materials reach from liquid water to amorphous and crystalline glasses like fused silica or sapphire.

The transparent behavior of dielectric materials rapidly changes when those are interacting with highly intense laser pulses. Although the energy of a single $800 \mathrm{~nm}$ photon, carrying an energy of $1.55 \mathrm{eV}$, is much smaller than a typical band gap of $9 \mathrm{eV}$ for a dielectric, at intensities in the Tera- or Petawatt per $\mathrm{cm}^{2}$ regime, nonlinear interactions become probable. While transient nonlinear effects like the optical Kerr effect (section 4.4) are only present as long as a highly intense laser pulse is incident, strong-field excitation of electrons from the valence to the conduction band (section 3.1) can have a more sustainable impact onto the material itself as we will discuss later.

\section{Sapphire}

Crystalline sapphire is the dielectric investigated in this work, that comes closest to a model-like system. With its large band gap of more than $9 \mathrm{eV}$ and the availability of ultra-pure (UV-grade) samples that have an ultra low density of impurities, it is perfect for investigating the excitation dynamics on a long time scale of several picoseconds [22]. The absence of impurities has two major advantages: First, impurities can lead to the presence of electrons in conduction band and second, they might introduce additional recombination effects that strongly reduce the lifetime of the electrons in the CB [23-25].

\section{Fused silica}

In addition to crystalline sapphire, we used amorphous fused silica $\left(\mathrm{SiO}_{2}\right)$ as an additional target sample. It is also a model material of a variety of dielectric materials in which exciton trapping is a 
dominant process. Trapping of excitons describes the creation of semi-bound states of a conduction band electron at a silicon site and a corresponding valence-band hole at a neighboring oxygen site in addition to a lattice relaxation [25] usually located within the original band gap. The most pronounced excitonic state is located at $5.2 \mathrm{eV}[25]$ above the valence band edge.

\section{Liquid water}

While sapphire and fused silica are perfect examples for dielectrics and often used targets for material processing, water is usually more exotic. Nevertheless, as it is the most essential molecule of life on earth, the dynamics of its excitation with ultrashort laser pulses has to be considered when tissue or cells are processed or laser ablation of solid materials in a liquid environment is performed, a few examples are given in [26-30].

Classically, a simplified model of an amorphous semiconductor was adapted to describe the electronic structure of water when discussing its excitation by short laser pulses, using a fixed band gap of $6.5 \mathrm{eV}$. However, this picture has changed over the last decades [31]. Elles et al. summarized the important mechanisms [32], highlighting both, ionization and dissociation processes in the excitation of liquid water. The range of excitation energies to promote electrons from the valence to the conduction band ranges from $8.3 \mathrm{eV}$ to $12.4 \mathrm{eV}$. One specialty of water is the presence of so-called solvated electrons. These represent a special structure of eight water molecules surrounding a water molecule with a solavted electron. Such a structure has a reduced band gap of $6.5 \mathrm{eV}$, however the number of solvated electrons, and thus the number of carriers that can be promoted via this channel is strongly limited [31].

To identify a single value of the band gap of water, that is the most probable in our experiments, we have to take a look on our spectral interference measurements (part [III). In these we use $800 \mathrm{~nm}$ laser pulses to create free electrons in water. From the number of created conduction band electrons and the resulting changes in the optical properties (see section 4.1) created via multi-photon excitation (described in the next section), a power law ${ }^{1}$ dependence on the incident pump fluence of the order $n$ is expected:

$$
n=\frac{E_{\mathrm{G}}+E_{\mathrm{Ponderomotive}}}{E_{\mathrm{Ph}}} .
$$

$E_{\mathrm{G}}$ is the band gap-, $E_{\text {Ponderomotive }}$ the ponderomotive-, and $E_{\mathrm{Ph}}$ the photon energy. The ponderomotive energy shift, that can be interpret as an pump intensity dependent increase in the band gap energy that can lead to higher order multi-photon excitation processes is introduced and discussed in section 3.1.1. Thus, it must be ensured that the photonicity is obtained from a measurement in a pump intensity range in which the increase in band gap does not lead to an increase in photonicity (i.e. above a ponderomotive energy of $1.55 \mathrm{eV}$ ). In figure 13.2 the phase shift, which is a measure of the conduction-band-electron density $\left.\right|^{2}$ in dependency on the pump pulse fluence is shown. The slopes for different temporal pulse shapes indicates a power law of $n=6$ up to a phase shift of $1 \mathrm{rad}$. Therefore, it fits to a band gap value of $8.3 \mathrm{eV}$. Spectral-absorption measurements done by Thomsen et al. [33] also reveal a strong absorption rise close to $150 \mathrm{~nm}$, equal to an energy of $8.3 \mathrm{eV}$. Furthermore, we modeled the excitation by using $8.3 \mathrm{eV}$ and obtained a very good agreement between simulations and experiment, shown and discussed in part III.

\footnotetext{
${ }^{1}$ At the lowest possible intensity above the excitation threshold.

${ }^{2}$ Valid assumption until a phase shift of $1 \mathrm{rad}$ (See part III)
} 


\section{Excitation mechanisms}

We mentioned, that the high intensity of an incident laser pulse can lead to an excitation, i.e. the promotion of electrons from the valence band to the conduction band. In general, these mechanisms can be divided into two categories: strong-field (SFI) and impact-/avalanche ionization (AI). Please note that we adapt the terminology of atomic-physics and therefore equally use the terms excitation/ionization and free/conduction-band electrons throughout this thesis.

\subsection{Strong-field excitation}

Starting at the lowest incident laser intensity with a photon energy smaller than the band gap, multi-photon excitation (MPI) is the first process 11 that is able to promote an electron from the valence- to the empty conduction band by the simultaneous absorption of several photons as shown in figure 3.1 . The number of photons required depends on the band gap- $E_{\mathrm{G}}$, the energy of the photon $E_{\mathrm{Ph}}$ and the ponderomotive energy as introduced in equation 2.1. Multi-photon processes of higher order require higher laser intensity compared to lower order multi-photon processes since the excitation probability decreases drastically with increasing number of involved photons [34, 35]. With increasing laser intensity, another strong-field process becomes probable, tunnel ionization (TI). The strong electric field of the laser pulse "bends" the potential of the atom such that the possibility that an electron is located outside is non-zero, i.e. the electron can tunnel through the coulomb barrier, as sketched in figure $3.1 \mathrm{~b}$.

A theoretical model for strong-field ionization, developed by Keldysh [34], provides a parameter ${ }^{2}$ that distinguishes between MPI and TI. The Keldysh parameter, $\gamma$, is given by [34]:

$$
\gamma=\frac{\omega_{1}}{e} \sqrt{\frac{c \varepsilon_{0} m E_{\mathrm{G}} n_{0}}{I}}
$$

where $c$ is the speed of light in vacuum and $\varepsilon_{0}$ the vacuum permittivity. $m$ is the electron mass, $I$ the incident, cycle-averaged laser intensity, $\omega_{1}$ the frequency and $n_{0}$ the linear refractive index of the irradiated dielectric material. In figure 3.2 the calculated Keldysh parameter $\gamma$ (blue line) is plotted as a function of the peak intensity $I$ for sapphire. It can be seen that $\gamma$ decreases with increasing pump pulse intensity. It is assumed that above a Keldysh parameter of $\gamma=1.5$, MPI is the dominant process whereas for values below $\gamma=1$ (horizontal black, dashed lines) MPI can be approximated by tunnel ionization [34,36].

The energy required for the excitation, does actually consists of two contributions: The energy of the band gap and the ponderomotive energy.

\footnotetext{
${ }^{1}$ Please note that at photon energies much lower (much higher frequencies) than used in our experiments, tunnel ionization becomes very probably even at low intensities.

${ }^{2}$ called Keldysh or adiabatic parameter
} 


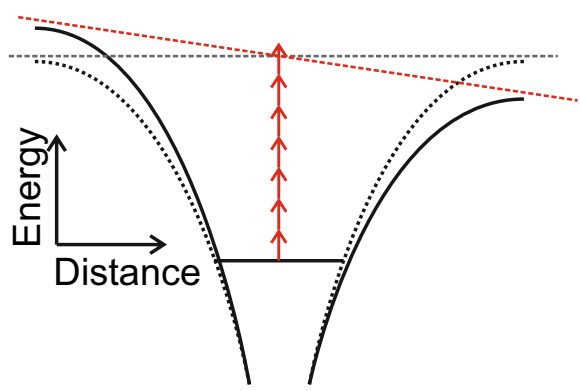

b

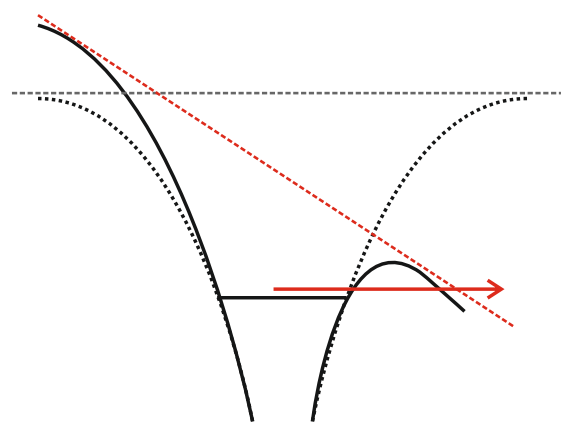

Figure 3.1.: Schematics of the strong-field ionization processes for a single atom. The black dotted lines show the coulomb potential when no external electric field is present, whereas the solid line shows the perturbed potential by an external laser field. The grey dashed line indicates the ionization potential. In a, the multi-photon ionization process is shown, where several photons are absorbed at once. $\mathbf{b}$ shows the tunnel ionization process, in which the Coulomb barrier is reduced by the electric field of the laser pulse allowing the electron to tunnel through the barrier. The ionization potential when a light field is present is indicated by the red dashed lines.

\subsubsection{Ponderomotive energy and band gap increase}

The ponderomotive energy shift is caused by the oscillations of the electrons in the conduction band that are driven by the oscillating electric field of the incident laser pulse and is given by [37]:

$$
E_{\text {Ponderomotive }}=\frac{e^{2} I}{2 m_{c} n_{0} \varepsilon_{0} \omega_{1}^{2}},
$$

where $m_{c}$ is the mass of the conduction band electron. The additional energy to the CB electrons act as an effective increase of the band gap energy [35,37]:

$$
\tilde{E}_{\mathrm{G}}=E_{\mathrm{G}}+E_{\text {Ponderomotive }}
$$

In figure 3.2 the effective band gap $\tilde{E}_{\mathrm{G}}$ (red, dashed line) is shown for typical peak intensities used in our experiments. As it can be seen, even at these moderate intensities, the effective band gap significantly supersedes the unperturbed one, leading to higher order MPI processes [35].

\subsection{Avalanche excitation}

Once an electron was promoted from the valence- (VB) to the conduction band (CB), it absorbs single photons from the light field of the exciting laser pulse via inverse-Bremsstrahlung. If a $\mathrm{CB}$ electron has gained sufficient energy, it can impact excite a second electron from the VB into the $\mathrm{CB}[35,38,39]$. The required, critical, energy $E_{\text {Crit }}$ is, taking into account the energy and momentum conservation, given according to Ridley [40] by:

$$
E_{\text {Crit }}=\frac{1+2 \mu}{1+\mu} \tilde{E}_{G}
$$




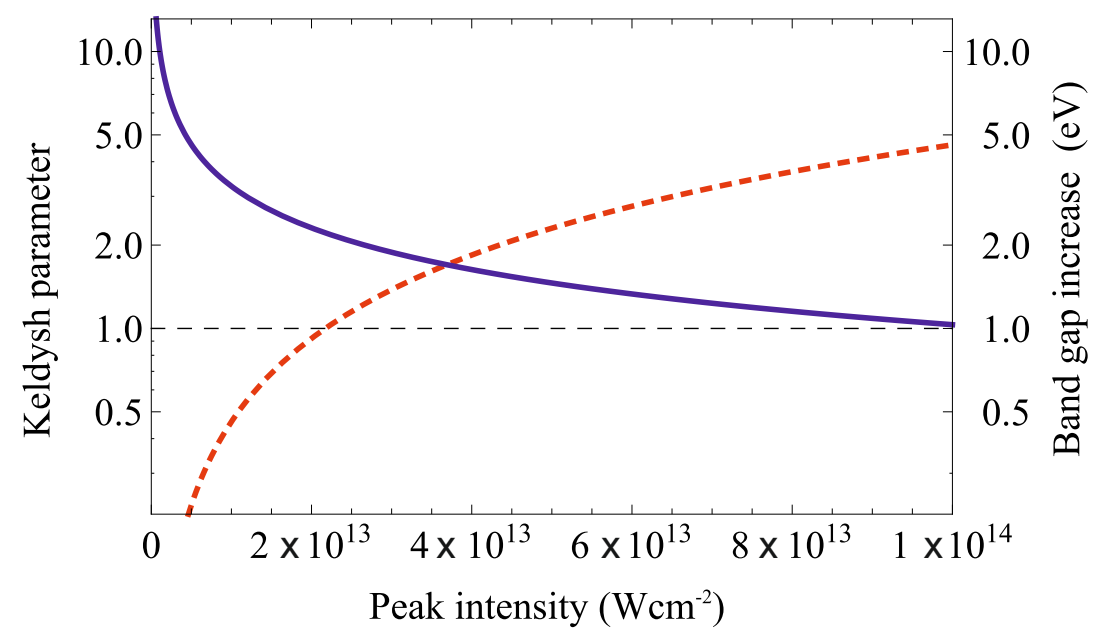

Figure 3.2.: Logarithmic representation of the calculated Keldysh parameter (blue line) $\gamma$ and band gap increase (red, dashed line) in dependence on the peak intensity for an $800 \mathrm{~nm}$ laser pulse in sapphire $\left(n_{0}=1.74, E_{\mathrm{G}}=9.9 \mathrm{eV}, m=0.36 m_{e}\right)$. The horizontal black, dashed line represents a value of 1 for the Keldysh parameter, indicating the transition to the tunnel excitation regime.

where $\mu=\frac{m_{C}}{m_{V}}$ is the ratio of the electron masses $\left(m_{\mathrm{C}}\right.$ and $\left.m_{\mathrm{V}}\right)$ within the conduction and the valence band, respectively. If both masses are equal, the critical energy is $E_{\text {Crit }}=1.5 \tilde{E}_{\mathrm{G}}$.

At the end of the avalanche excitation process both electrons will be located close to the conduction band bottom and the process can start all over again, if the exciting laser pulse is still incident. The multiplicity of this excitation process might cause an cascade of excitations, giving the origin for the name "avalanche excitation".

In comparison to MPI, which is instantaneous, the avalanche process is delayed [28, 41, 42]. The reason is the finite acceleration time of the conduction-band electrons. When a laser pulse is incident, the electron is accelerated, gaining energy via inverse Bremsstrahlung [35]. Since the electron is inside a highly dense material, it will scatter. This can either be carrier-carrier or carrier-phonon scattering [35]. Therefore, the limiting factor is the time in which the electron is accelerated, under the assumption that between each scattering event one photon is absorbed [28]. These scattering times are in general in the order of femtoseconds [11, 28, 39, 43, 44]. To gain enough energy for impact excitation, the electron will therefore scatter several times.

\subsection{Intraband-thermalization}

Up to this point, we have discussed the two pathways for an electron to be promoted from the VB to the $\mathrm{CB}$. Although the bottom of the $\mathrm{CB}$ is the lowest possible and first energetic state an electron will reach by the lowest order MPI, not all electrons are located in it:

Due to the free carrier absorption, adding the energy of a single photon from the pump pulse, electrons are distributed across the conduction band as sketched in figure $3.33^{3}$. The peak closest to the lower CB edge corresponds to the MPI, whereas peaks at higher energies correspond to $n+1$

\footnotetext{
${ }^{3}$ The distribution of the holes in the VB is very similar.
} 

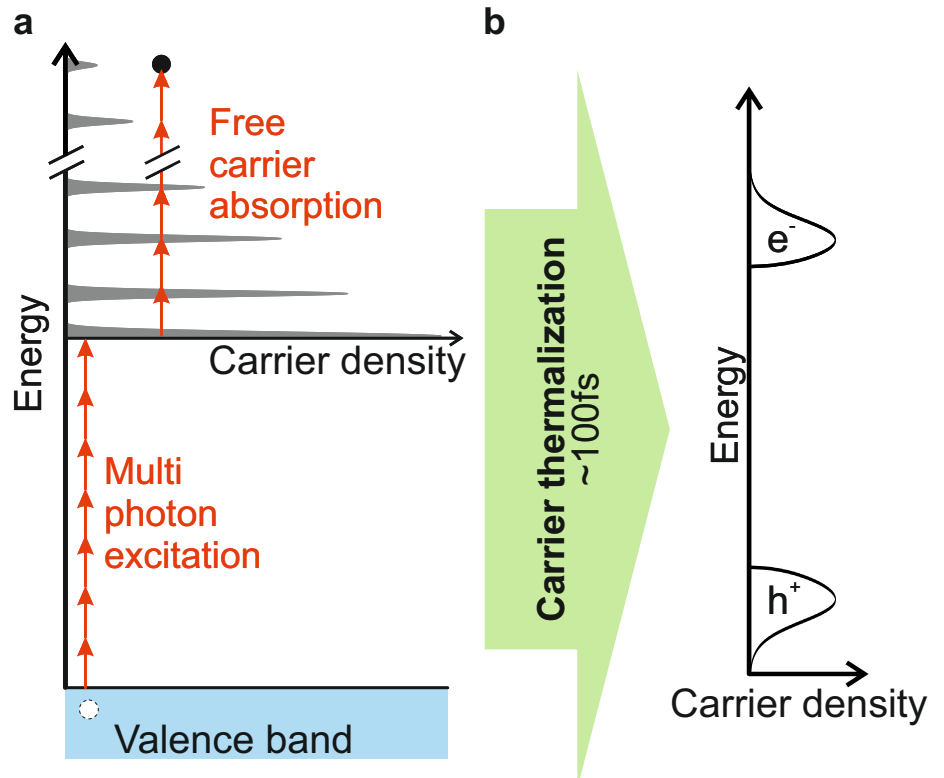

Figure 3.3.: a, The excitation and heating of electrons leads to a discrete-like distribution of electrons in the conduction band. b. Intraband-thermalization causes the initially discrete distribution into one that can be described by a quasi-Fermi-distribution.

absorption's of photons provided by the pump pulse. The time at which the electron distribution has such a shape is either during the excitation or shortly after, for a very short pump pulse, as scattering quickly spreads the distribution of electrons, followed by intraband-thermalization. "Intraband" indicates that electrons in the valence band and the holes in the conduction band are equilibrating among themselves first [45, 46].

For a material in thermodynamic equilibrium, the energetic distribution of electrons and holes is usually described by a Fermi- or Boltzmann distribution with a single temperature and chemical potential (the band gap energy) [21]. However, this is very different in case of a laser-excited dielectric. Shorty after its excitation it is in a highly non-equilibrium state as written above.

The resulting distribution that follows the intraband-thermalization process can be described by a quasi-Fermi distribution, having separate temperatures and chemical-potentials for holes and electrons [45, 46]. Such a distribution of electrons and holes is sketched in figure $3.3 \mathrm{~b}$. The way in which such distributions can be calculated is described in chapter 6 .

\subsection{Relaxation processes}

After electrons have been ejected into the conduction band, their lifetime is limited: Depending on the recombination and relaxation process, the electrons will transfer their energy to the lattice and finally relax back into the VB on a time scale of hundreds of picoseconds. While the bandto-band recombination is a transition from the $\mathrm{CB}$ into the $\mathrm{VB}$, associated with either an transfer of the energy to the lattice or a radiative recombination visible as fluorescence, the self trapping of electrons into self -trapped exciton states (STEs) is a special effect occurring in a certain class of dielectrics which includes fused silica (see chapter 2). While only a fraction of the energy is transferred to the lattice (providing energy for the hole to get into the higher excitonic state), the 


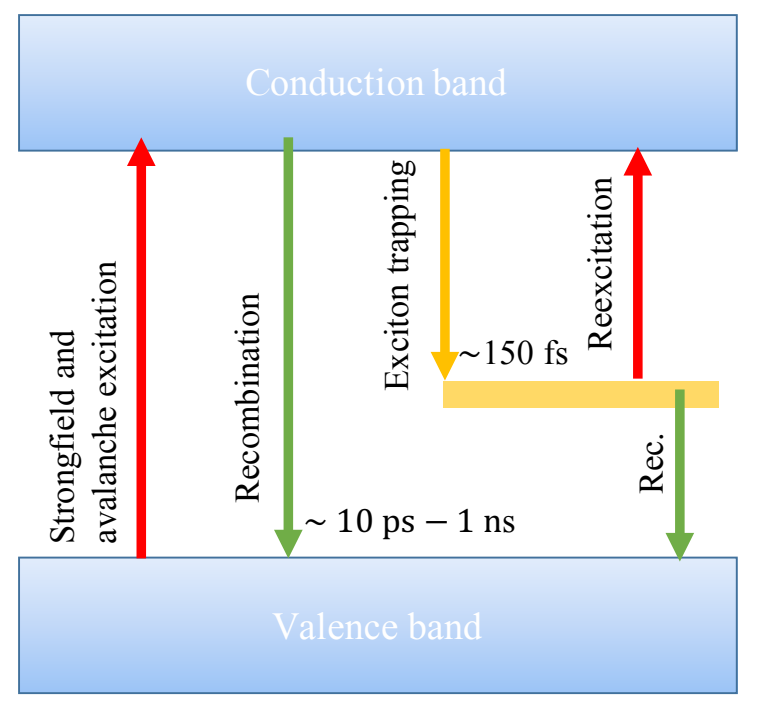

Figure 3.4.: Sketch of excitation and recombination processes in band gap materials with indications of typical time scales.

trapped exciton is located within the band gap and can, on a time-scale of nanoseconds [47] relax into the VB or be transferred into so-called color states which are permanent imperfections within the lattice of the material [24]. These might lead to the absorption of certain light frequencies and let the material appear to have a certain color in the human visibility spectrum, giving the origin for the name of the defect. The processes described above are sketched in figure 3.4, indicating typical timescales (if applicable).

A further recombination process that can occur is Auger-recombination: Similarly to the bandto-band recombination, a $\mathrm{CB}$ electron recombines with a hole from the $\mathrm{VB}$, however in this case, the energy is transferred to another CB electron, putting it into a high-energy state [48, 49]. This might allow a re-excitation of another electron via impact excitation, making Auger-recombination and avalanche excitation competing processes. We do not account for Auger recombination in the simulations involved in this work. 



\section{Optical properties of laser-excited dielectrics}

The excitation of a dielectric material, i.e. the injection of carriers into the conduction band, drastically changes the optical properties. In this chapter we briefly describe how the complex dielectric function depends on the number of carriers and how light is attenuated when propagating through such a material:

\subsection{Drude model}

The plane wave solution for an electromagnetic wave propagating through a dispersive medium in $z$-direction is given by a solution of Maxwells equations [37, 50]:

$$
E(t, z)=E_{0} \exp \left(i\left(\frac{\tilde{n} \omega_{1} z}{c}\right)-\omega_{1} t\right) \hat{e}_{y}
$$

where $\tilde{n}$ is the complex refractive index, $c$ the speed of light in vacuum, $\omega_{1}$ the frequency of the laser pulse, $E_{0}$ the peak amplitude of the oscillating electric field at $z=0$ and $\hat{e}_{y}$ the polarization vector.

The complex refractive index $\tilde{n}$, is given by the dielectric function (under the assumption $n_{0}=$ $\sqrt{\varepsilon \mu} \approx \sqrt{\varepsilon}$, where $\mu$ is the permeability of the material) $\varepsilon[37]$ :

$$
\tilde{n}^{2}=\varepsilon=\varepsilon_{\mathrm{r}}-i \frac{\sigma}{\varepsilon_{0} \omega_{1}}
$$

with the relative permittivity $\varepsilon_{\mathrm{r}}$, the vacuum permittivity $\varepsilon_{0}$ and the conductivity $\sigma$ of the material. Please note, that this equation is only valid under the assumption that the relative permittivity of the material is constant and does not depend on the conduction band electron density. That this is not always the case is discussed later in this section.

For an unexcited dielectric material, in which no electrons are present in the conduction band $N=0(\sigma=0)$ the dielectric function is given by the relative permittivity $\varepsilon_{\mathrm{r}}$. In case of an excited dielectric, the quasi-free electrons lead to a non-zero conductivity of the material.

The contribution to the changes of the permittivity can e.g. be described by the Drude model. The equation of motion of the electrons driven by the external electric field of the laser pulse $E(t)=E_{0} \exp \left(-i \omega_{1} t\right)$ with the laser frequency $\omega_{\mathrm{l}}$ is given by [37, 50]:

$$
m_{\mathrm{c}} \frac{\partial^{2} x}{\partial t^{2}}+\frac{m_{\mathrm{c}}}{\tau} \frac{\partial x}{\partial t}=-e E(t)
$$




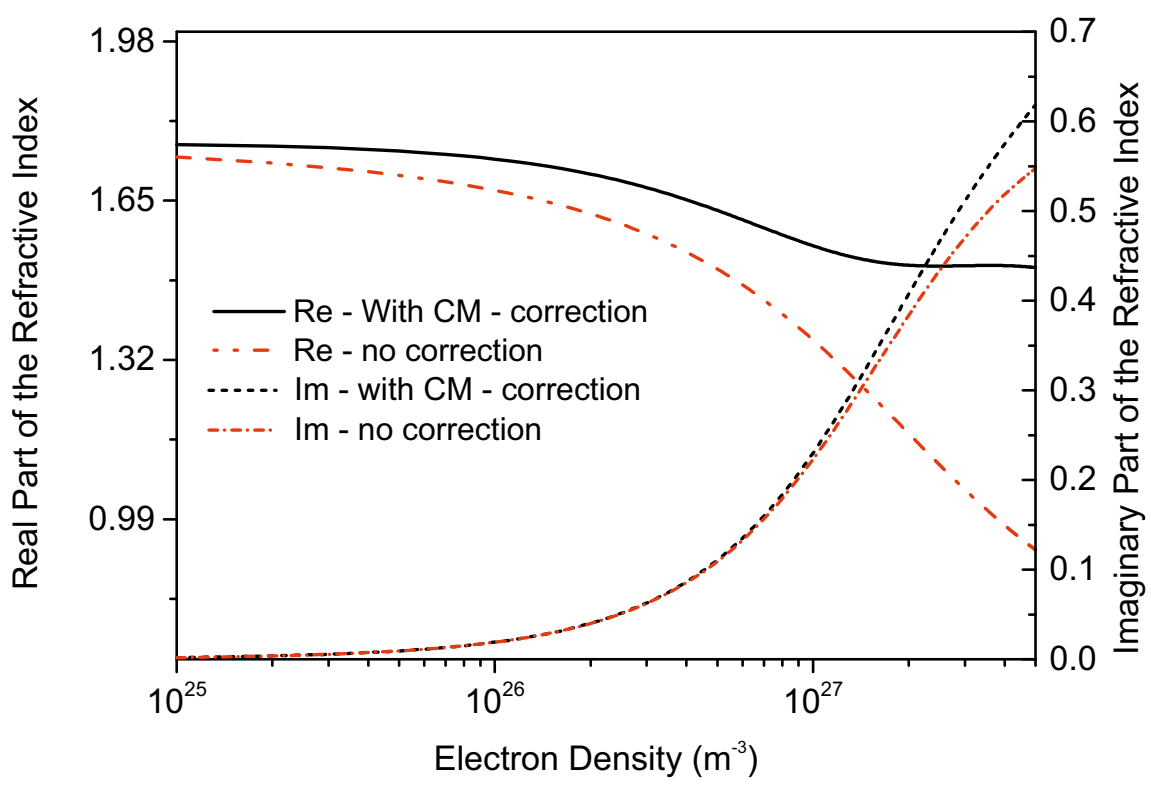

Figure 4.1.: Calculated real and imaginary part of the complex refractive index in dependence on the CB carrier density, with and without the Clausius-Mossotti (CM) correction for sapphire with a scattering time coefficient of $\tau^{*}=1 \mathrm{fs}$. (Other values used for the calculation can be found in table 19.1.

with the conduction band electron mass $m_{\mathrm{c}}$, the electron scattering time $\tau$ and the charge of the electron $e$.

By Fourier transforming the equation of motion and solving it, we obtain:

$$
m\left(-i \omega_{1}\right)^{2} x\left(\omega_{1}\right)+m_{C} \tau^{-1}\left(-i \omega_{1}\right) x\left(\omega_{1}\right)=-e E\left(\omega_{1}\right),
$$

which can be simplified to:

$$
\left(-m \omega_{1}^{2}-i \omega_{1} m_{C} \tau^{-1}\right) x\left(\omega_{1}\right)=-e E\left(\omega_{1}\right) .
$$

This finally gives the solution for the displacement:

$$
x\left(\omega_{1}\right)=-\frac{e}{m_{C}} \frac{E\left(\omega_{1}\right)}{-i \omega_{1} \tau^{-1}} .
$$

We now calculate the dipole moment of the charge displacement $(x)$ via the definition $\mu\left(\omega_{1}\right)=$ $-q x\left(\omega_{1}\right)$, where $q$ is the charge of the particle, i.e. the elementary charge $e$.

This provides:

$$
\mu\left(\omega_{1}\right)=\frac{e^{2}}{m_{C}} \frac{E\left(\omega_{1}\right)}{-\omega_{1}^{2}-i \omega_{1} \tau^{-1}} .
$$

Now we use the volume averaged dipole moment to obtain the polarization $P\left(\omega_{1}\right)$ of the material [37]:

$$
P\left(\omega_{1}\right)=\frac{1}{V} \Sigma \mu_{i}\left(\omega_{1} a\right)=N\left\langle\mu\left(\omega_{1}\right)\right\rangle=\varepsilon_{0} \chi\left(\omega_{1}\right) E\left(\omega_{1}\right),
$$

where $\mathrm{N}$ is the number of electrons in the dielectric and $\chi\left(\omega_{1}\right)$ is the susceptibility given as:

$$
\chi\left(\omega_{1}\right)=\frac{N e^{2}}{\epsilon_{0} m_{C}}\left(-\omega_{1}^{2}-i \omega_{1} \tau^{-1}\right)^{-1} .
$$


The first part:

$$
\omega_{\mathrm{pl}}=\sqrt{\frac{N e^{2}}{\varepsilon_{0} m_{c}}},
$$

is called the plasma frequency.

Thus, the complex dielectric function $\varepsilon\left(\omega_{\mathrm{pl}}\right)$ of the excited dielectric material is finally given by [37, 50]:

$$
\varepsilon\left(\omega_{\mathrm{l}}\right)=\varepsilon_{\mathrm{b}}-\frac{\omega_{\mathrm{pl}}^{2}}{\omega_{1}^{2}+i \frac{\omega_{1}}{\tau}}
$$

$\varepsilon_{\mathrm{b}}$ is the contribution by the valence band electrons and usually set to $\varepsilon_{\mathrm{b}}=n_{0}^{2}$, where $n_{0}$ is the realvalued, linear refractive index of the material. The conductivity of the excited material, introduced above, can be directly obtained from the imaginary part of the complex dielectric function.

\subsection{The Clausius-Mosotti correction}

The approximation of a constant contribution to the dielectric function by the valence band electrons, $\varepsilon_{\mathrm{b}}$ is only valid up to a certain density of excited electrons. Due to their promotion, the permitivity generated by the remaining valence electrons decrease [51]. A correction is given by [51,52]:

$$
\varepsilon_{\mathrm{b}}=1+\frac{3 N_{\mathrm{V}} \beta_{\mathrm{CM}}}{3-N_{\mathrm{V}} \beta_{\mathrm{CM}}}
$$

where $N_{\mathrm{V}}=N_{\text {Max }}-N$ is the number of valence band electrons, $N_{\text {Max }}$ the total number of available electrons ${ }^{1}$ and $\beta_{\mathrm{CM}}$, which can be obtained from the simple condition that in the case of no excitation $N=0$, the equation simply has to be solved by $\varepsilon_{\mathrm{b}}=n_{0}^{2}[51]$ :

$$
\beta_{\mathrm{CM}}=\frac{3}{N_{\mathrm{Max}}} \frac{n_{0}-1}{n_{0}+2}
$$

The changes, accompanied by the CM correction of the refractive index are important in the high excitation regime, as can be seen in figure 4.1. Here, real and imaginary part of the complex refractive index in dependence on the conduction-band-electron density are shown, with and without the $\mathrm{CM}$ correction. At high electron densities, the real part strongly decreases in the uncorrected case. For the corrected value, the behavior is different. Close to the critical electron density $\left(N_{\mathrm{C}}(800 \mathrm{~nm}) \approx 10^{27} 1 / \mathrm{m}^{3}\right)$ the decrease flattens. The critical density is defined as the value at which the plasma frequency is equal to the optical frequency of the laser pulse and thus defined as:

$$
N_{\mathrm{C}}=\frac{\omega_{1} m_{\mathrm{C}} \varepsilon_{0}}{e^{2}}
$$

The imaginary part, which is solely responsible for absorption (see eq. 5.11), is less influenced by the reduction of the valence band electrons. Only above the critical density $\left(N>10^{27} \frac{1}{\mathrm{~m}^{3}}\right)$ the uncorrected value underestimates the imaginary part that takes into account the correction.

\footnotetext{
${ }^{1}$ This number is equal to the density of valence band electrons of the unexcited material. Values for sapphire and water can be found in the tables 19.1 and 20.1
} 


\subsection{Lorentz-Drude Model}

In materials that express exitonic states, such as fused silica (chapter2), the Drude model (equation 4.11) has to be extended to the Lorentz-Drude model. As excitonic states are located within the band gap, they are characterized by a specific resonance energy $\hbar \omega_{1}$ and a corresponding line width $\hbar \Gamma_{\text {exc }}$. The dielectric function that takes into account the contribution by CB electrons and electrons located in the excitonic states is given by [21, 22, 37, 44, 50]:

$$
\varepsilon\left(\omega_{\mathrm{l}}\right)=\varepsilon_{\mathrm{b}}-\frac{\omega_{\mathrm{pl}}^{2}}{\omega_{1}^{2}+i \frac{\omega_{1}}{\tau}}+f_{\mathrm{exc}} \frac{\omega_{\mathrm{exc}}^{2}}{\omega_{1}^{2}-\omega_{1}^{2}+i \omega_{1} \Gamma_{\mathrm{exc}}},
$$

where $\omega_{\text {exc }}^{2}$ is similarly defined to the plasma frequency as:

$$
\omega_{\mathrm{exc}}^{2}=\sqrt{\frac{e^{2} N_{\mathrm{exc}}}{m_{\mathrm{exc}} \varepsilon_{0}}}
$$

with the number of excitons $N_{\text {exc }}$ and their mass $m_{\text {exc }}$.

\subsection{Optical Kerr effect}

In addition to the change of the refractive index by the CB electrons and STEs, the transient optical Kerr effect (OKE) plays an important role, especially if the material is probed when the pump pulse is still incident. In isotropic (inversion symmetric) materials, where a varying electric field is incident, the electric susceptibility is given by (up to the third order) [3]:

$$
\chi \approx \chi^{(1)}+\frac{3}{4} \chi^{(3)} E(t)^{2}
$$

A detailed derivation of the susceptibility and discussion can be found in the PhD thesis of Tilmann Kalas available on the group server. In most cases, in which the nonlinear susceptibility is smaller than $n_{0}^{2}=1+\chi^{(1)}$ the refractive index, can be expressed in terms of a Taylor series by:

$$
\tilde{n} \cong n_{0}+n_{2} I(t)
$$

with the linear- $n_{0}$ and nonlinear refractive index $n_{2}$. The complex reflective index including the transient $\mathrm{OKE}$ and the contribution by free carriers is given by:

$$
\tilde{n}=\sqrt{\varepsilon_{b}-\frac{\omega_{\mathrm{pl}}^{2}}{\omega_{1}^{2}+i \frac{\omega_{1}}{\tau}}}+n_{2} I(t)
$$

As this nonlinear effect is only present as long as the driving pulse is incident it can be used to characterize one of the pulses as shown in the appendix in section 16.1.2. 


\section{a}

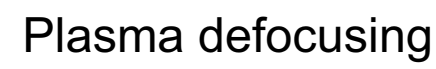

b Balance of defocusing $\mathrm{c}$ and self-focusing

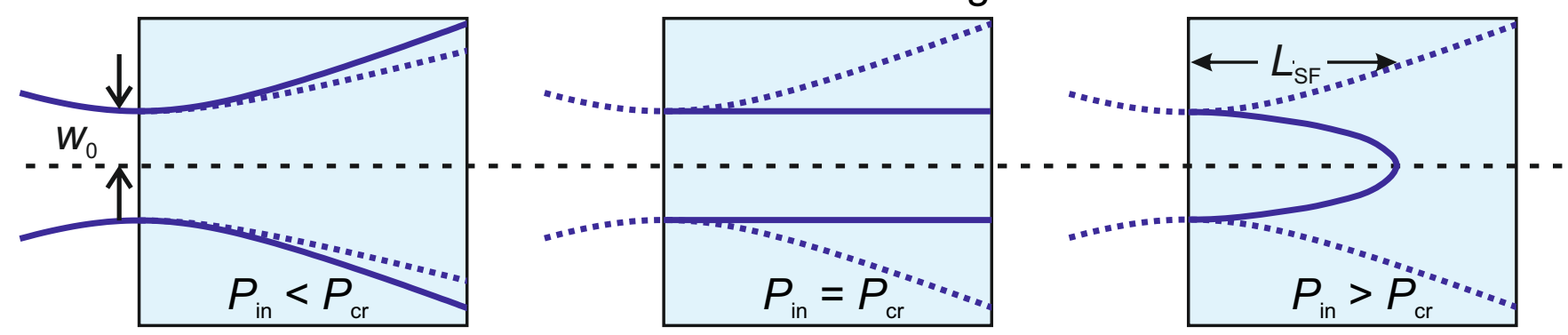

Figure 4.2.: a, Sketch of plasma-defocusing in case that the incident power is less than the critical value, but strong enough to create a free electron plasma. b, Sketch of the counterbalancing case. $\mathbf{c}$, Sketch of beam collapse in the case of filamentation.

\subsection{Propagation effectcs}

Due to the intensity dependence of the OKE, a typical laser pulse with a Gaussian radial intensity distribution can be affected by self-focusing: The intensity profile will cause a positive refractive index change being highest in the center. This resembles, for $n_{2}>0$, a focusing lens. Depending on the incident power and the nonlinear refractive index, the laser beam might decrease in radius (see figure 4.2p) and eventually collapses (see figure 4.2p) and lead to a filament. The critical power $P_{\text {cr }}$ required for self-focusing to overcome the natural diffraction is given for a Gaussian beam by [53]:

$$
P_{\text {cr }}=\frac{3.77 \lambda^{2}}{8 \pi n_{0} n_{2}}
$$

where $\lambda$ is the wavelength of the laser pulse. While the change of the refractive index by the OKE is dependent on the incident intensity, the usage of a critical power seems to be inconvenient. However, the "virtual" lens argument justifies the usage of a critical power:

Lets assume an incident laser pulse has sufficiently high intensity for self focusing. When the power is kept constant but the beam radius is doubled, the intensity drops by one quarter and therefore the induced changes to the refractive index by the OKE are only of one quarter. However, due to the much larger area, the lens is larger and compensates the reduced changes to the refractive index changes [54].

The typical length $L_{\mathrm{SF}}$ over which an intense laser beam collapses due to self focusing is given by [53]:

$$
L_{\mathrm{SF}}=\frac{2 n_{0} w_{0}}{\lambda_{0} \sqrt{\frac{P_{\mathrm{in}}}{P_{\mathrm{cr}}}-1}},
$$

where $P_{\text {in }}$ is the incident power of the laser pulse and $w_{0}$ the beam waist. When the beam collapses, the intensity of the laser pulse inside the material drastically increases due to the decreasing beam radius. This will lead to an excitation of electrons by strong-field processes as described in section 3.1. As we have seen above, $\mathrm{CB}$ electrons will actually decrease the dielectric function and therefore the refractive index (see eq. 4.11), acting as a defocusing lens. This effect is called plasma-defocusing [53] and can happen as soon as CB electrons are created and thus also even if a power below the one required self focusing is applied, sketched in figure $4.2 \mathrm{a}$. After the collapsed 
pulse is defocused by the excitation of carriers, it might have still sufficient intensity to lead to a subsequent collapse. This periodic process and the resulting propagation of the laser pulse is called filamentation [53]. 


\section{Modeling laser excitation}

A basic way to model the laser excitation is the use of a single rate equation model to calculate the time dependent conduction-band-electron density induced by ultrashort laser pulses. It was initially developed by Stuart et al., based on a Fokker-Planck-Equation, including strong-field excitation mechanisms [55,56]. In general, all single-rate-equation (SRE) models used to calculate the time dependent conduction-band-electron density $N(t)$ are described by a differential equation in the form of:

$$
\frac{\partial N(t)}{\partial t}=N_{\mathrm{SFI}}+\eta_{\mathrm{AI}}
$$

The first part is the contribution by the strong-field ionization (SFI) and the second part the contribution by avalanche ionization (AI), respectively. As mentioned in the introduction, there exist a variety of expressions for the different ionization mechanisms. In part III, we use [12, 28, 39, 42, 54, 57, 58]:

$$
\frac{\partial N(t)}{\partial t}=\sigma_{n} I(t)^{n}+\alpha_{\mathrm{AI}} I(t) N(t)
$$

where $N(t)$ is the time dependent conduction-band-electron density, $\sigma$ a multi-photon coefficient, which order $n$ depends on the integer number of photons (equation 2.1) to overcome the band gap energy $E_{\mathrm{G}} \cdot \alpha_{\mathrm{AI}}$ is the avalanche excitation coefficient.

Usually the contribution by strong-field excitation is calculated by the Keldysh formalism [34. 59]. The full Keldysh expression provides an intensity depending transition from multi-photon to tunnel ionization. It also includes the increasing photoniticity due to the ponderomotive shift. The expression can be reduced to either pure multi-photon or tunnel ionization [34].

Even if the full Keldysh formalism is often used to calculate the excitation by strong-field ionization, we use a more simple approach, in which a constant multi-photon coefficient is fitted to match experimental results, as done in a manifold of other studies as well [11, 12, 28, 60-62]. It is important to note that this simplification does not account for higher order multi-photon excitations caused by ponderomotive energy shift, explained in section 3.1.1. In figure 3.2, the band gap increase in sapphire due to the ponderomotive energy shift is shown for a typical peak intensity range used in our experiments. As it can be seen, the band gap increase is even at low intensities already on the order of the photon energy $(1.5 \mathrm{eV})$.

The contribution by avalanche ionization has been assumed to have a constant factor $\alpha_{\mathrm{AI}}$ of proportionality (as used in 5.2). Kennedy on the other hand used an expression based on the classical plasma absorption rate $W_{\mathrm{PL}}$ given by $[22,42,52,63]$ :

$$
W_{\mathrm{PL}}=\frac{I}{\hbar \omega_{1}} \frac{2 \kappa \omega}{c} \frac{1}{N}
$$

where $\kappa$ is the imaginary part of the refractive index, given by:

$$
\tilde{n}=k+i \kappa,
$$


with the real part of the refractive index $k$. In comparison to a constant excitation rate, the plasma absorption rate by Kennedy not only takes into account the laser pulse frequency, it also considers the absorption coefficient of the material that is consistently changing during and after the laser excitation.

Although the amount of conduction band electrons created via avalanche excitation might be different when using a constant avalanche coefficient compared to the approach by Kennedy, we justify its use due to the simplicity and reduced computational effort. Furthermore we obtain $\alpha_{\mathrm{AI}}$ in our investigations as a fitting coefficient by comparing simulations with experimental results.

As discussed in the previous section, the inverse-Bremsstrahlung absorption is a process that suffers from scattering after each absorption of a photon and therefore the electron takes a certain time to reach the critical energy [28]. A way to implement this retardation is to use the so-called multiple-rate-equation model, developed by Rethfeld et al. [64] and extended by Christensen et al. [65]. Here the conduction band is discretized into a certain amount of conduction band states, each separated by the photon energy. The transition probability between each level is calculated by the above mentioned plasma absorption/electron heating rate (eq. 5.3). Thus the energetic distribution of the electrons in the conduction band is given by a quasi Fermi distribution and a natural retardation is implemented [22, 52]. This approach is very accurate, but on the other hand it requires a high computational effort in order to calculate the transitions between a high number of states. This is further discussed in section 5.2 .

In our simulations we implement the natural retardation of the avalanche excitation by a method introduced by Vogel and coworkers [28]. By making use of a retarded time $t_{\text {Ret }}$ for the electron density in equation 5.2, it will be taken into account that the electrons, that contribute to the impact excitation process at the present time, have been excited at an earlier time. The retarded time $t_{\text {ret }}$ is given by [28]:

$$
t_{\mathrm{ret}}=t-t^{*}
$$

where $t^{*}$ is the time the electron requires to gain sufficient energy:

$$
t^{*}=n_{\mathrm{c}} \tau
$$

where $n_{\mathrm{c}}=\left\langle\frac{E_{\text {Crit }}}{E_{\mathrm{Ph}}}\right\rangle$ is the number of photons required to access the critical energy and $\tau$ the scattering time, which is mostly assumed to consists of the carrier-carrier and carrier-phonon scattering time [35, 52], respectively. The latter one is obtained experimentally whereas $\tau^{*}$ is used as a fitting parameter, as discussed in the next section.

Finally, we end up with a differential equation for the time dependent electron density in the form [11,28]:

$$
\frac{\partial N(t)}{\partial t}=\frac{N_{\max }-N}{N_{\max }}\left(\sigma_{n} I(t)^{n}+\alpha_{\mathrm{AI}} I(t) N\left(t_{\mathrm{ret}}\right)\right) .
$$

$\frac{N_{\max }-N}{N_{\max }}$ is a scaling factor that includes the starvation of the excitation processes in case all valence band electrons $\left(N_{\max }\right)$ were excited.

\subsection{Scattering time}

As seen in the last sections, the scattering time has an large impact on the optical properties of the excited dielectric, but also to the excitation process. Often [11, 12, 38, 39, 66-69], the scattering 


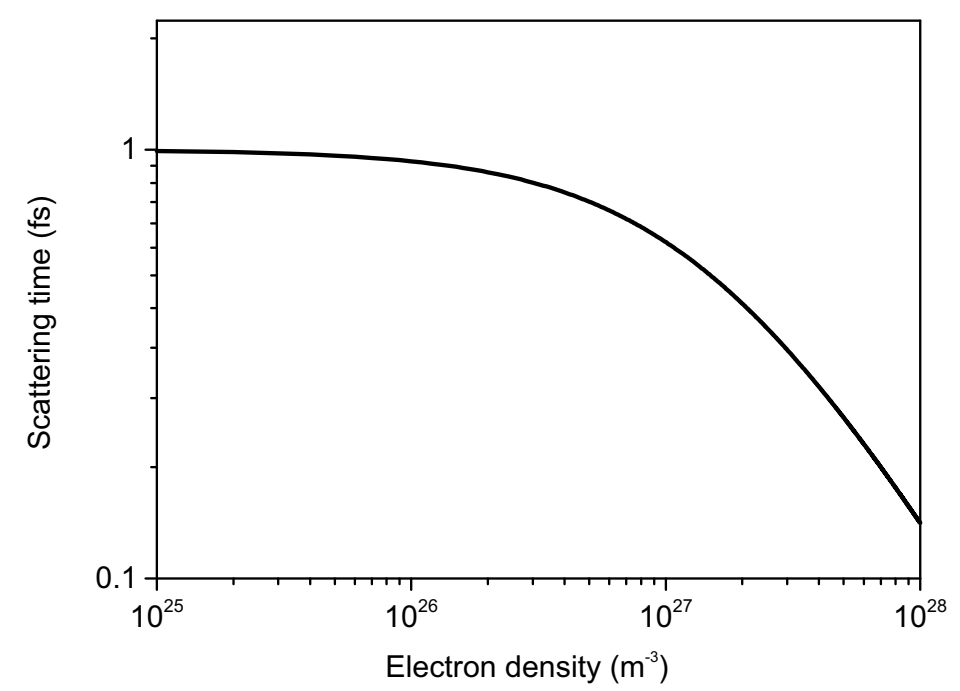

Figure 5.1.: Electron density dependent scattering time for a scattering time coefficient $\tau^{*}=1 \mathrm{fs}$ and a carrier-phonon scattering time $1 / \gamma_{c-p}=1 \mathrm{fs}$.

time is assumed to be constant, which is only true for low excitation cases [35, 44, 52, 70]. In this regime, the carrier-phonon scattering is the dominant process. Increasing the strength of excitation and therefore the density of $\mathrm{CB}$ electrons and their kinetic energy, the velocity of the CB electrons and thus the probability of scattering rises. The scattering time $\tau_{\mathrm{s}}$ can be expressed as a sum of scattering rates $\gamma$ :

$$
\frac{1}{\tau_{\mathrm{s}}}=\gamma_{\mathrm{s}}=\gamma_{c-p}+\gamma_{c-c}
$$

which consists of a constant term for the carrier-phonon $\gamma_{c-p}$ and an energy dependent carriercarrier scattering rate $\gamma_{c-c}$. While the carrier-phonon scattering time is a value that can be obtained experimentally, as shown in [11,12], the carrier-carrier scattering rate is used as a fit parameter by us. However, it is possible to approximate it by [52]:

$$
\frac{1}{\tau_{c-c}}=\gamma_{c-c}=4 \pi \sqrt{2} r^{2} v N
$$

where $v$ is the root-mean-square velocity of the electrons and $r$ the electron radius. This radius is either the Debye screening length for moderate to high electron densities or the de-Broglie wavelength for low electron densities. The detailed description of the used model can be found in [52].

For reasons of simplicity, we use an expression introduced by Siegel et al. [71], in which the electron scattering time is related to the critical electron density $N_{\mathrm{C}}$ and the conduction-bandelectron density $N[71]$ :

$$
\tau_{c-c}=\frac{1}{\gamma_{c-c}}=\tau^{*} \frac{N_{c}}{N},
$$

where $\tau^{*}$ is a fitting parameter, referred in this work to "scattering time coefficient". It defines the scattering time for an electron density equal to the critical density. The scattering time dependence on the electron density can be seen in figure 5.1. Here, at a very low excitation around $10^{25}$ to $10^{26}$ $\mathrm{m}^{-3}$ the scattering time is equal to the carrier-phonon scattering time whereas higher values of the electron density leads to a drastic decrease in scattering time, theoretically down to the sub-fs regime. 


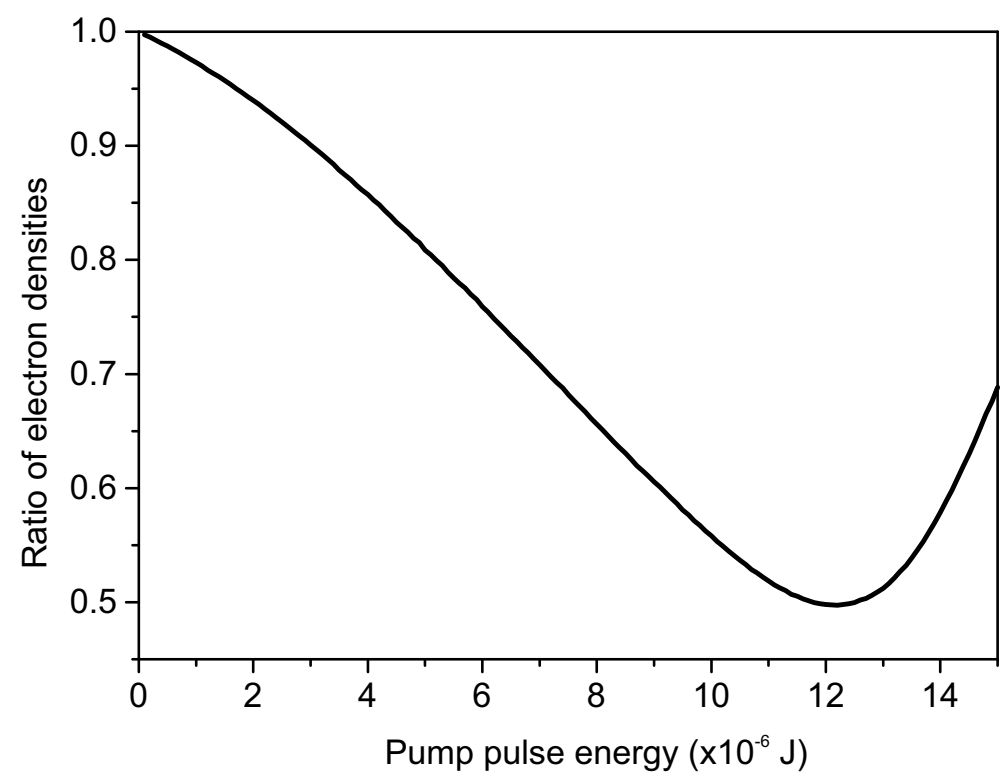

Figure 5.2.: Calculated ratio of the $\mathrm{CB}$ electron density in water, created by a positive TAP, with a high and low retardation time, as explained in the text. Values for this calculation of water can be found in table 20.1 .

Unfortunately, a variable scattering time will lead to a changed retardation of the avalanche ionization process (eq. 5.7) in our model described above. The change occurs as long as the electron density changes, causing a problem in the computation. Modern, software-integrated, nonlinear differential equation solver, which uses a Runge-Kutta-Method, like Mathematica or Matlab are not able to solve delayed differential equations with changing delay. Therefore, the electron density dependent scattering time $\tau_{\mathrm{s}}=1 / \gamma_{\mathrm{s}}$ will be used to model the optical properties, whereas we use a fixed retardation time for the electron excitation. The consequences affects only pump pulses with a longer pulse duration, since short and ultrashort laser pulses $(<50 \mathrm{fs})$ mainly addresses multi-photon ionization [11, 60, 62, 72]. Laser pulses with longer pulse duration, creating a CB density higher than the critical value will experience the decreasing retarded time (equation 4.14). Therefore, the contribution by the avalanche ionization process at high excitation intensities might be underestimated.

To estimate the effect of the decreasing retardation time at high electron densities, we calculated the intensity depending electron density using a positive TAP laser pulse, described in section 1.2 , with the retardation time given by the carrier-photon scattering time and the scattering time at the critical electron density. The calculation is based on the parameter summarized in table 20.1. The quotient of the final conduction band electron densities using the high and low retardation time in dependence on the pump pulse energy is shown in figure 5.2. It can be seen that a small retardation time will lead to a higher electron density compared to a high value (ratio $<1$ ). Since the electron density evolves while the pulse is incident, the low scattering time will be reached after a certain time and is not present when the pulse arrives. Therefore, the presented case is assumed to be the worst case scenario.

In table 5.1 the introduced time expressions are summarized. 


\begin{tabular}{|l|l|l|}
\hline Temporal quantity & Symbol & Typical time scales \\
\hline Time & $t$ & - \\
Retarded time & $t_{\mathrm{R}}=t-n_{\mathrm{c}} \tau_{\mathrm{s}}$ & - \\
Scattering time & $\tau_{\mathrm{s}}=1 / \gamma_{\mathrm{s}}=1 /\left(\tau_{e-e}+\tau_{e-p}\right)$ & $\left(10^{-14}-10^{-16}\right) \mathrm{s}$ \\
Scattering time coefficient & $\tau^{*}$ & $\left(10^{-15}-10^{-16}\right) \mathrm{s}$ \\
Carrier-carrier collision time & $\tau_{c-c}=1 / \gamma_{e-e}=\tau^{*} \frac{N_{\mathrm{C}}}{N}$ & - \\
Carrier-phonon collision time & $\tau_{c-p}=1 / \gamma_{e-p}$ & $\left(10^{-14}-10^{-15}\right) \mathrm{s}$ \\
\hline
\end{tabular}

Table 5.1.: Overview of introduced time expressions, where $n_{\mathrm{c}}$ it the number of photons needed to reach the critical energy $E_{\text {Crit }}, N$ is the conduction-band-electron density and $N_{\mathrm{C}}$ the critical electron density.

\subsection{Multiple-Rate-Equation model}

While the simulations performed to obtain the results presented in the third part (III) of this thesis, are based on the simple rate equation approach described above, we required an extended multiple rate equation model to calculate the excitation of sapphire for the understanding of laser amplification in dielectrics, presented in part III.

In the (extended-) multiple rate equation model [44,70], originally introduced by Rethfeld [64], the conduction band is discretised into many energy levels, which are described by their kinetic energy and the density of electrons that is located in each "level" (see figure 3.3). The model not only calculates the strong-field excitation of electrons from the valence- to the conduction band via the Keldysh description, but also the inverse-Bremsstrahlung absorption within the conduction band. Thus, the electrons promoted by strong-field excitation into e.g. the lowest level in the $\mathrm{CB}$, will be promoted to higher levels by the absorption of single photons from the pump pulse, providing kinetic energy to the electron (i.e. the energy of the photons absorbed). Therefore, the model also predicts how many electrons will reach a sufficiently high level in the conduction band to impact ionize further electrons 3.2. Overall, this model does not only give a more adequate calculation of a total carrier density, it also provides the total kinetic energy of the electrons in the conduction band as discussed in chapter 6. The detailed description of the modeling, i.e. a set of coupled differential equations and the modeling parameter is given in [44,70].

\subsection{Modelling laser pulse propagation}

In chapter 4, we briefly described how the excitation of electrons into the conduction band will lead to a complex valued dielectric function, i.e. an absorbent material. The absorption is usually quantified by the absorption coefficient $\alpha$, given by:

$$
\frac{2 \kappa \omega_{1}}{c}=\alpha
$$

being the inverse penetration depth $\frac{1}{\alpha}$ of the material at a certain light frequency $\omega_{1}$ [37]. Please note that this rough approximation is only valid if the penetration depth is much shorter than the spatial extend of the laser pulse. For the unexcited dielectric material, the absorption coefficient is zero. With an increasing electron density the material is transformed into a transient metallic state causing absorption of the pulse. 

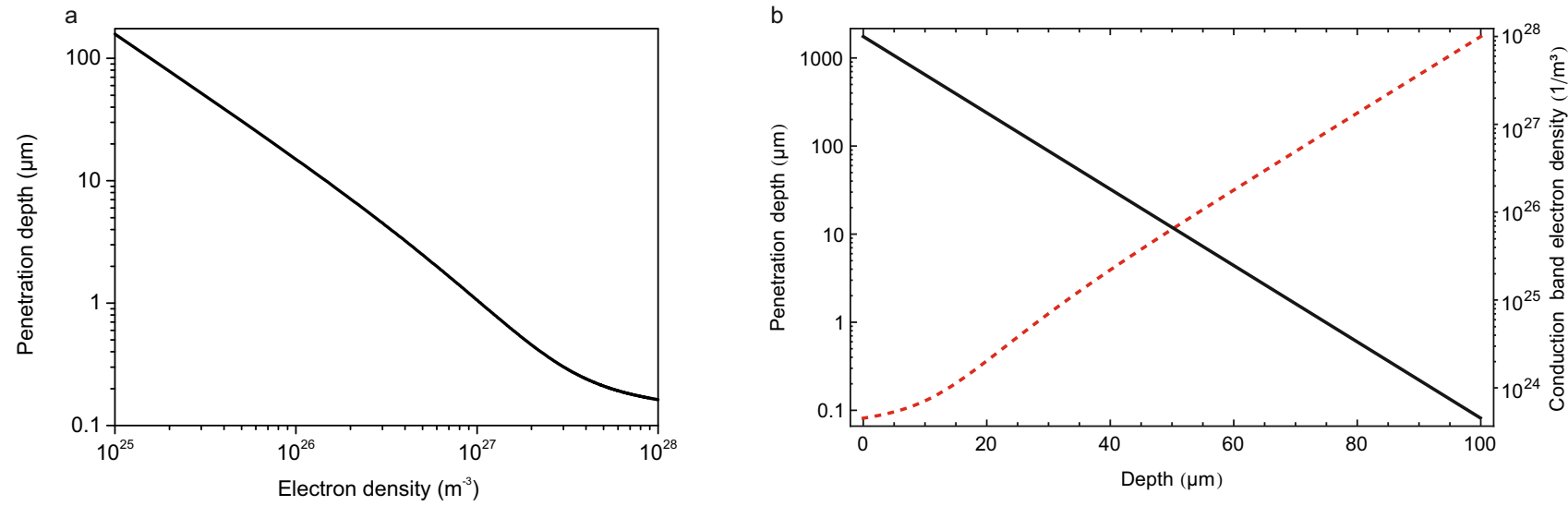

Figure 5.3.: a, Calculated penetration depth of an $800 \mathrm{~nm}$ pump pulse in dependence on the electron density for a scattering time coefficient $\tau^{*}$ and a carrier-phonon scattering time $\tau_{c-p}$ of $1 \mathrm{fs}$. b, Linear-logarithmic representation of the conduction-band-electron density (black line) and the resulting, calculated (using same parameters as in a), penetration depth (red, dashed line) of an $800 \mathrm{~nm}$ pump pulse as a function of depth.

In figure 5.3 a, the penetration depth is shown as a function of the $\mathrm{CB}$ electron density. The depth strongly decreases with increasing electron density. A penetration depth is actually only applicable if the electron density would be constant over the whole thickness of a sample, like in a metal. As the electron density drastically changes with respect to the depth, we waive to use it. However, it is possible to average the penetration depth, shown in 5.3 , to obtain an "effective penetration depth". In the example shown in figure $5.3 \mathrm{~b}$, we obtain a value of $175 \mu \mathrm{m}$ for the plotted depth-dependent conduction-band-electron density.

In addition to the described metallic-like absorption, the change in the refractive index will lead to a changed reflection coefficient $R$ which can be calculated by the Fresnel equation at a normal incidence of the laser pulse [37,71]:

$$
R=\left|\frac{n_{1}-\tilde{n}}{n_{1}+\tilde{n}}\right|^{2}=\left|\frac{k_{1}-k_{2}}{k_{1}+k_{2}}\right|^{2},
$$

with the linear refractive index $n_{0}$ of the surrounding material (in our case air $n_{1}=n_{\text {Air }}=1$ ), the complex refractive index of the target material $\tilde{n}$ or in terms of the wave vector $(k)$ :

$$
\begin{gathered}
k_{1}^{2}=\frac{\omega_{1}^{2}}{c^{2}} n_{\text {Air }}^{2}, \\
k_{2}^{2}=\frac{\omega_{1}^{2}}{c^{2}}(\tilde{n})^{2}=\frac{\omega_{1}^{2}}{c^{2}}\left(n_{0}^{2}-\frac{N e^{2}}{\omega_{1}^{2} m_{\mathrm{c}} \varepsilon_{0}\left(1+\frac{i}{\omega_{1} \tau}\right)}\right),
\end{gathered}
$$

where $1-R=T$ defines the transmission of the incident laser pulse through the surface of the sample:

$$
I(t, z=0)=(1-R) I_{0} \exp \left(-4 \ln 2\left(\frac{t-t_{0}}{\Delta t}\right)^{2}\right) .
$$

It is important to note that only a discontinuous change of the refractive index will cause a reflection and therefore it only affects the boundary of the material. As the electron density is continuously 


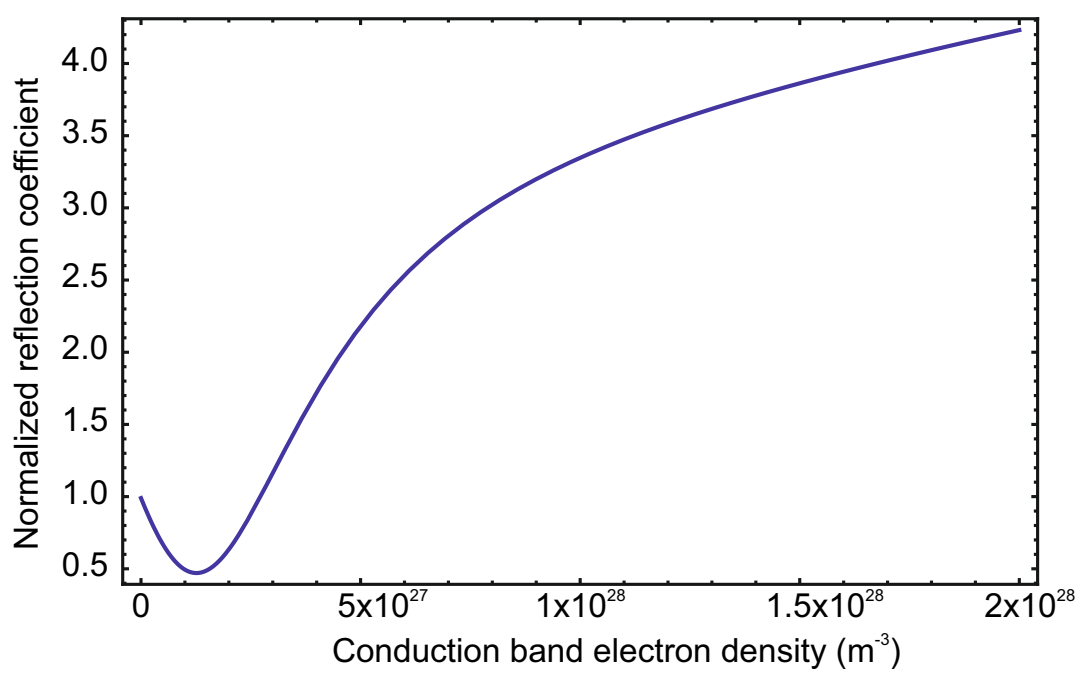

Figure 5.4.: Calculated conduction-band-electron density dependent reflection coefficient of an $800 \mathrm{~nm}$ pulse at the surface of a sapphire sample with a scattering time coefficient of $\tau^{*}=1 \mathrm{fs}$ and a carrier-phonon scattering time of $1 / \gamma_{c-p}=1 \mathrm{fs}$. Please note that we normalized the reflection coefficient to the value in case of no excitation $(R=0.0345)$.

distributed along the propagation direction of the laser pulse, no additional reflection take place within the sample.

In general the reflection coefficient of a dielectric is constant, like for example at an air/water ${ }^{1}$ interface for a wavelength of $800 \mathrm{~nm}$ it is given by equation 5.12 to be $R=0.0345$. In figure 5.4 the normalized, calculated reflection coefficient in dependence on the density of CB electrons is shown $(\tilde{n}(N))$. It can be seen, that with increasing density the reflection coefficient decreases down to a minimum followed by an increases to values higher than the initial one [71].

Beside the reflection of the laser pulse at the front surface, the excitation of carriers will lead to absorption by two mechanisms: the multi-photon excitation itself and free carrier absorption (inverse-Bremsstrahlung) by the created conduction band electrons. As an example for the first process imagine the excitation of a single electron by six-photon multi-photon process. Thus, these six photons must be missing from the pump pulse after the excitation.

The resulting intensity dependence with respect to the propagation depth is given by [37, 52]:

$$
\frac{\partial I(t, z)}{\partial z}=-\alpha I(t, z)-N_{\mathrm{SFI}} \frac{N_{\mathrm{Max}}-N}{N_{\mathrm{Max}}} \frac{\tilde{E}_{\mathrm{G}}}{I(t, z)}
$$

where $\alpha$ is the absorption coefficient of the material being dependent on the excitation conditions as discussed above. $N_{\mathrm{SFI}}$ is the strong-field excitation rate, introduced in equation 5.10 and $\tilde{E}_{\mathrm{G}}$ is the corrected band gap, accounting for the band gap and the ponderomotive energy shift. The term $1 / I(t, z)$ is used to obtain the absorption per unit length.

If we neglect, for a moment, the absorption by excitation we obtain a differential equation:

$$
\frac{\partial I(t, z)}{\partial z}=(1-R(t)) \alpha I(t, z)
$$

\footnotetext{
${ }^{1}$ The properties of water are summarized in table 20.1 .

${ }^{2}$ Could be approximated by $\sigma_{n} I^{n}$ as in our simplified rate equation model.
} 
where the solution is given, assuming a constant $\alpha$, by the well known Lambert-Beer-Law $(I(t, z)=$ $I(t) \times \exp (-\alpha z))$. Unfortunately, the absorption coefficient is not constant in an excited dielectric $\alpha \rightarrow \alpha(z)$, but a function of different parameters like the conduction band electron density or the scattering time. However, by dividing the material into very "thin" discrete slices of one nanometer thickness, the approximation of a constant absorption coefficient is valid and the free carrier absorption in each of these slides can be calculated via the Lambert-Beer-Law.

We now come back to the absorption due to the creation of carriers via multi-photon (and avalanche) excitation. Instead of using the formalism described in equation 5.16 we make use of a much more simple approch: the minimum-energy-per-area $\epsilon_{\text {Min }}$ or minimum fluence $F_{\text {Min }}$. This value describes the energy per area that was required to excite a certain amount of conduction band electrons at a respective band gap energy and is given by [73]:

$$
F_{\text {Min }}=\epsilon_{\min }=\Delta E_{\mathrm{G}} \int_{0}^{\mathrm{L}} N(z) d z .
$$

Each electron created in a layer of the material required an energy of $E_{\mathrm{G}}$ and thus this fraction of the pump pulse fluence must be missing in the subsequent layer of the material, neglecting additional energy due to the ponderomotive energy shift. We realize this by simply subtracting $F_{\text {Min }}$ from the pulse fluence leading to a lower intensity of the laser pulse. Please note that by doing so, the nonlinearity of the absorption process (the part by MPI) is not taken into account and thus this approach can only be considered as a rough approximation, which will become especially important when dealing with highly intense and ultrashort bandwidth limited laser pulses. However it is negligible when used for low excitation cases or when shaped pulses are used, like in part III.

Please note that we do not consider the transverse aspects of the spatial Gaussian beam propagation because in our experiments, we always use beam diameters such that the Rayleigh length is orders of magnitude larger than the sample thicknesses, ensuring a constant beam diameter over the complete length of the sample. Also, we do not consider any nonlinear propagation effects [74] which were introduced in section 4.5 .

\subsubsection{Exemplary calculations}

An example of this calculation is demonstrated in figure 5.6 and 5.7. It shows the time dependent electron density $N(t)$ in sapphire and peak intensity distribution $I(t)$ for a pulse of $50 \mathrm{fs}$ FWHM right below the surface $(L=0)$ and in the depth of $1 \mu \mathrm{m}$. In the case of a low intensity pump pulse (figure 5.6) the electron density is homogeneously distributed along the sample, as seen in the upper left inset and the intensity is nearly equal to the one right below the surface. In contrast to this, the highly intense pulse, which is two times higher than in low excitation case, it causes a highly non-homogeneous distribution along the propagation axis (upper left inset of fig 5.7). Even if the peak intensity is only two times higher, the resulting plasma density increases by five orders of magnitude. Furthermore the electron density decreases by about one order of magnitude over the first micrometer. Additionally, the pump pulse is strongly absorbed, especially after the peak intensity has passed, which is caused by absorption due to the free carrier absorption by the CB electrons. 


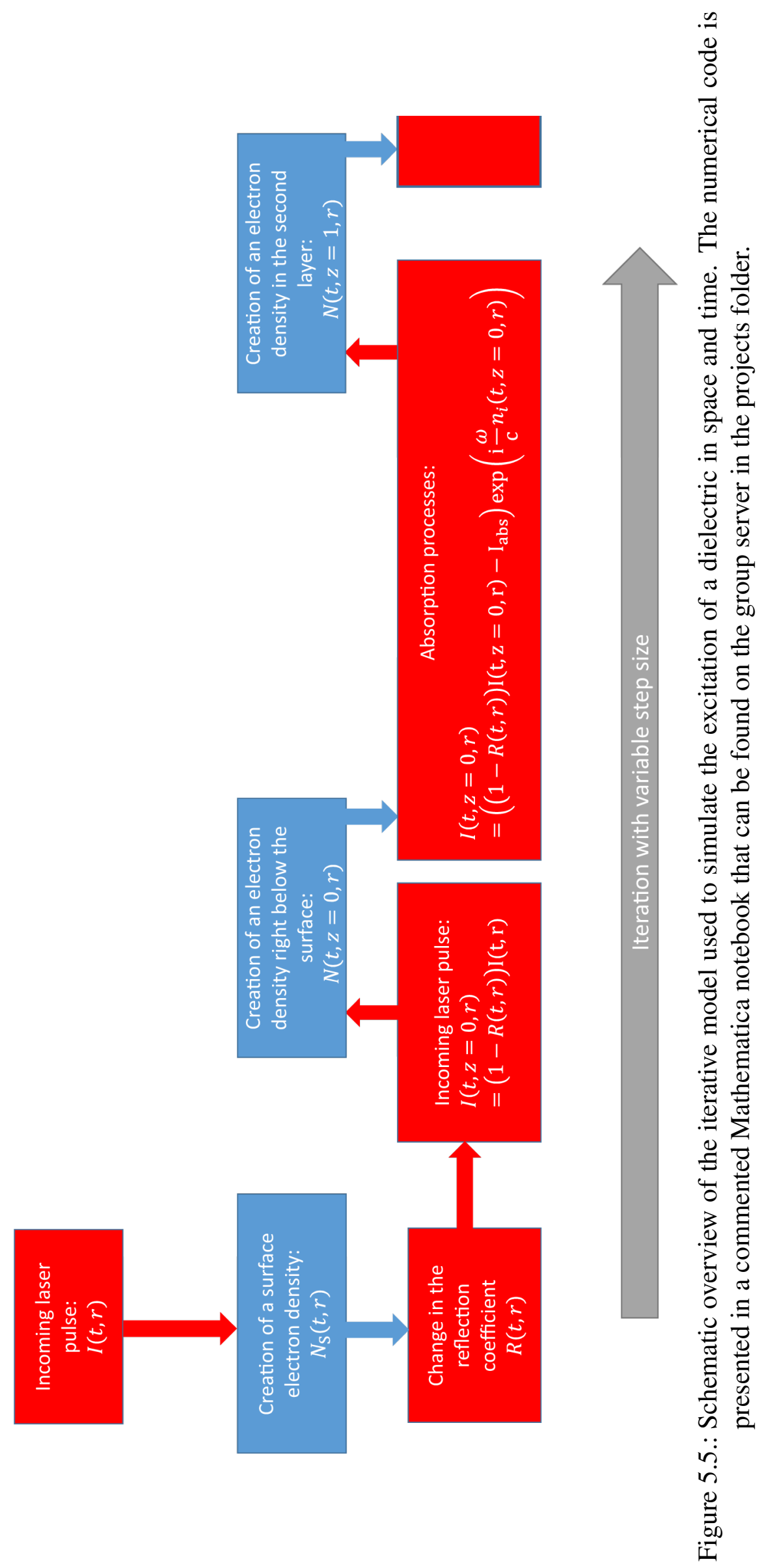




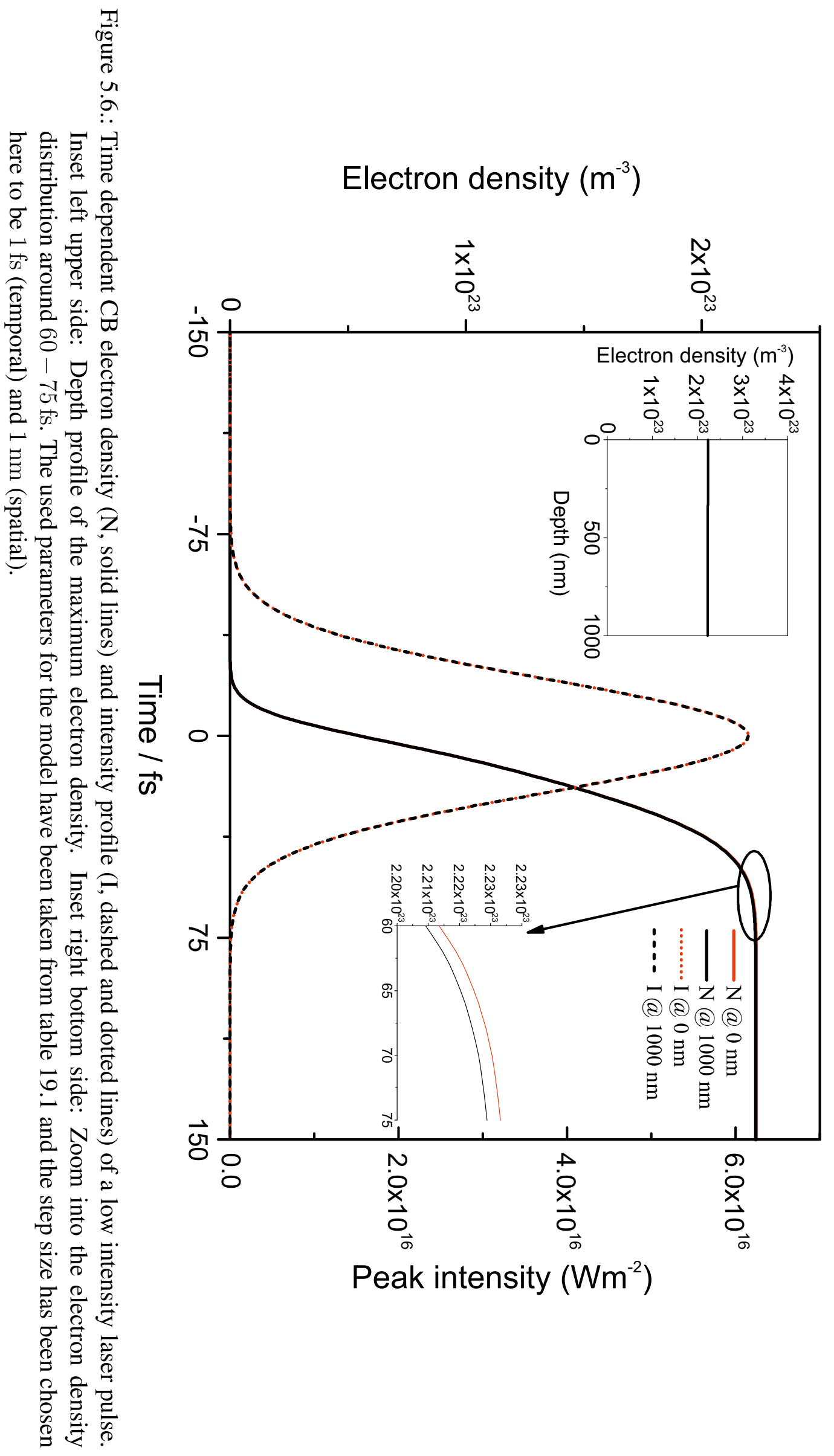




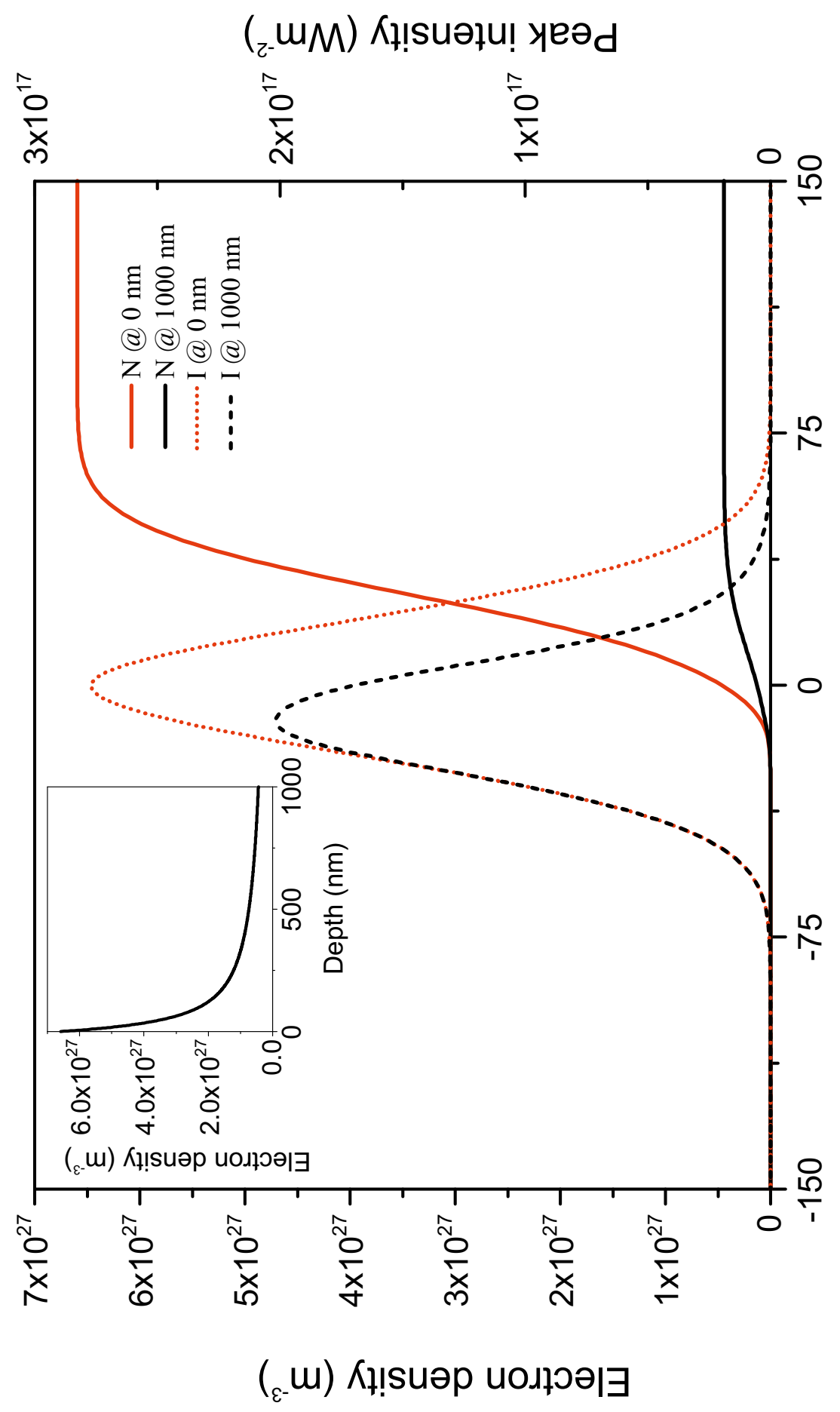

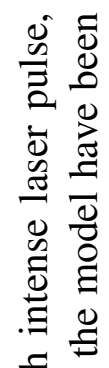

.00

范这

苍

ఫृ

ఫิ

寻

สิ

ปี

䒕 Ð

$\Xi . \Xi \pm$

进高望

Ф)

() $: 2$

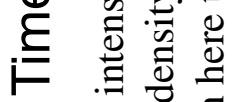

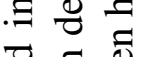

흐 을

के $\frac{0}{0}$

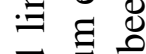

긍 丞

z

를 월

प्रेण

च

总

过

흉

के

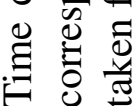

$\because$

in

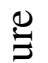

항 
To calculate the radial distribution of the electron density by using a Gaussian pump beam profile we simply use as an input for the simulation:

$$
I(t, r, z)=I(t)\left(\frac{w_{0}}{w_{0} \sqrt{\left(\frac{z}{z 0}\right)^{2}+1}}\right)^{2} \exp \left(-\frac{2 r^{2}}{\left(w_{0} \sqrt{\left(\frac{z}{z_{0}}\right)^{2}+1}\right)^{2}}\right)
$$

with $w_{0}$ the $1 / e^{2}$ beam waist in the focus, the Rayleigh range $z_{0}=\frac{\pi w_{0}^{2}}{\lambda}$, the radius $r$ and wavelength of the incident laser pulse $\lambda$. This part can be implemented as a first order approximation for low beam waists, such that the Rayleigh range is much smaller than the sample thickness. Please note that it is not a replacement for an accurate ray tracing model. 


\section{Modelling intraband thermalization after excitation and conditions of population inversion}

In section 3.3 we discussed that the initially discrete electron and hole distributions quickly thermalizes. Depending on the material properties, the intraband-thermalization can be finished within a few hundred femtoseconds or even faster [45, 46], eventually producing Fermi distributed electrons and holes. These are characterized by their specific temperature $T_{e / h}$ and chemical potential $\mu_{e / h}$. Both energy-dependent distributions are then given by [45, 46, 75]

$$
N_{e}(E)=\rho_{e}(E) f\left(E, \mu_{e}, T_{e}\right)
$$

for the conduction band electrons and by

$$
N_{h}(E)=\rho_{h}(E) f\left(E, \mu_{h}, T_{h}\right)
$$

for the holes in the valence band, using the three-dimensional density of states of an electron gas $\rho$ and the Fermi distribution function $f$. The equations are given by [21]:

$$
\begin{aligned}
\rho_{e}(E) & =\frac{8 \pi \sqrt{2}}{h^{3}} \times m_{e}^{\frac{3}{2}} \times \sqrt{E-E_{\mathrm{CB}}}, \\
\rho_{h}(E) & =\frac{8 \pi \sqrt{2}}{h^{3}} \times m_{h}^{\frac{3}{2}} \times \sqrt{E_{\mathrm{VB}}-E}, \\
f\left(E, \mu_{e}, T_{e}\right) & =\frac{1}{\exp \left(\frac{E-\mu_{e}}{k_{\mathrm{B}} T_{e}}\right)+1}, \\
f\left(E, \mu_{h}, T_{h}\right) & =\frac{1}{\exp \left(\frac{\mu_{h}-E}{k_{\mathrm{B}} T_{h}}\right)+1},
\end{aligned}
$$

where $E_{\mathrm{CB}}$ is the energy of the lower conduction band edge, $E_{\mathrm{VB}}$ the energy of the upper VB edge (usually set to $0 \mathrm{eV}$ ). $m_{e}$ and $m_{h}$ are the masses of the electron in the $\mathrm{CB}$ and the hole in the VB. $k_{\mathrm{B}}$ is the Boltzmann and $h$ the Planck constant.

The temperatures and chemical potentials for electrons and holes are determined by a two-dimensional root-finding problem [45, 46]. On the short time scale of several picoseconds, when no interband thermalization takes place, the total number of carriers and total kinetic energy is conserved.

Therefore, the kinetic energy and total number of carriers modelled by the pump-pulse excitation (see section 5.2) must be equivalent to the values described by the quasi Fermi distributions after the intraband-thermalization:

$$
\begin{aligned}
N_{e, M R E}=\int_{E_{\mathrm{CB}}}^{\infty} N_{e}(E) \mathrm{d} E & =\int_{E_{\mathrm{CB}}}^{\infty} \rho_{e}(E) f\left(E, \mu_{e}, T_{e}\right) \mathrm{d} E \\
E_{e, M R E}=\int_{E_{\mathrm{CB}}}^{\infty} E \cdot N_{e}(E) \mathrm{d} E & =\int_{E_{\mathrm{CB}}}^{\infty} E \rho_{e}(E) f\left(E, \mu_{e}, T_{e}\right) \mathrm{d} E .
\end{aligned}
$$


MRE indicates the total number of electrons and energy provided by the simulation based on the multiple rate equation model. Please note that there is an unique set of $\left(\mu_{e}, T_{e}\right)$ that solves both equations simultaneously [45, 46].

For the holes in the valence band the solution is given in a similar fashion by:

$$
\begin{gathered}
N_{h, M R E}=\int_{-\infty}^{E_{\mathrm{VB}}} N_{h}(E) \mathrm{d} E=\int_{-\infty}^{\mathrm{VB}} \rho_{h}(E) f\left(E, \mu_{h}, T_{h}\right) \mathrm{d} E, \\
E_{h, M R E}=\int_{-\infty}^{E_{\mathrm{VB}}} E N_{h}(E) \mathrm{d} E=\int_{-\infty}^{\mathrm{VB}} E \rho_{h}(E) f\left(E, \mu_{h}, T_{h}\right) \mathrm{d} E .
\end{gathered}
$$

A challenge that we encountered is that, in the current state, the extended MRE model [22, 44, 70] only accounts for the excitation of electrons and therefore we are unable to account for the energy deposited into the valence band and the exact number of holes created; a more elaborate model, i.e. a multiple-rate equation model for holes, would be required to fully solve this problem.

By making use of two approximations it is possible to obtain the required solutions:

- First, we assume that $N_{h, M R E}=N_{e, M R E}$, as each electron promoted into the CB via multiphoton excitation leave one hole in the valence band.

- Second, we assume equal masses for the electrons and holes, which allows us to estimate an equal temperature for electrons and holes [21] $\left(T_{e}=T_{h}\right)$ and thus to obtain $\mu_{h}$ from equation 6.9 .

As simple as it might sound to find the unique solution for the temperature and chemical potential for the electrons the more difficult it is when trying to find it in a suitable time. From the extended MRE model we obtain for different initial pump pulse fluences (30 different values) a time and depth (70 values) resolved map of conduction-band-electron density and total kinetic energy, shown in figure 6.1 a for the electron density. As we are, in this study, only interested in pumpfluence dependent dynamics that follow the excitation by the pump pulse, we took the results at a delay time step at which all excitation processes are finished.

Now we start with the first pump pulse fluence and for each simulated layer of the $131 \mu \mathrm{m}$ thick sample ${ }^{1}$, the above mentioned equations are solved. As it is for a program like Mathematica very time consuming to find the unique solution, we handled it differently:

We calculated the solutions of 6.7 and 6.8 for a selected conduction band edge and a large variety of chemical potentials and temperatures. These "lookup-tables" were then searched to find the couples of $T_{e}$ and $\mu_{e}$ at which $N_{e}$ and $E_{e}$ are closest to the values provided by the MRE at the given incident fluences and depth position. Depending on how "closest value" is defined (usually solutions within a few percent are accepted), several couples of $T_{e}$ and $\mu_{e}$ might be found. In a second step we select the couple(s) $\left(T_{e}, \mu_{e}\right)$ that solved $N_{e}$ and $E_{e}$ simultaneously. Depending on whether there are more than 1 solution, the "acceptance" margin can be reduced until a single solution is left. Finally the values for the holes by using $T_{e}=T_{h}$ and $N_{e}=N_{h}$ are obtained (as described above).

This process has to be repeated for each pump fluence setting and each of its 70 depth steps. One could think that it is maybe sufficient to link the results $T_{e}, \mu_{e}, T_{h}, \mu_{h}$ simply to a certain incident

\footnotetext{
${ }^{1}$ The sample was divided in 70 layers with different thickness, whereas the layer thickness increases with depth, taking into account the depth-dependent dynamics.
} 


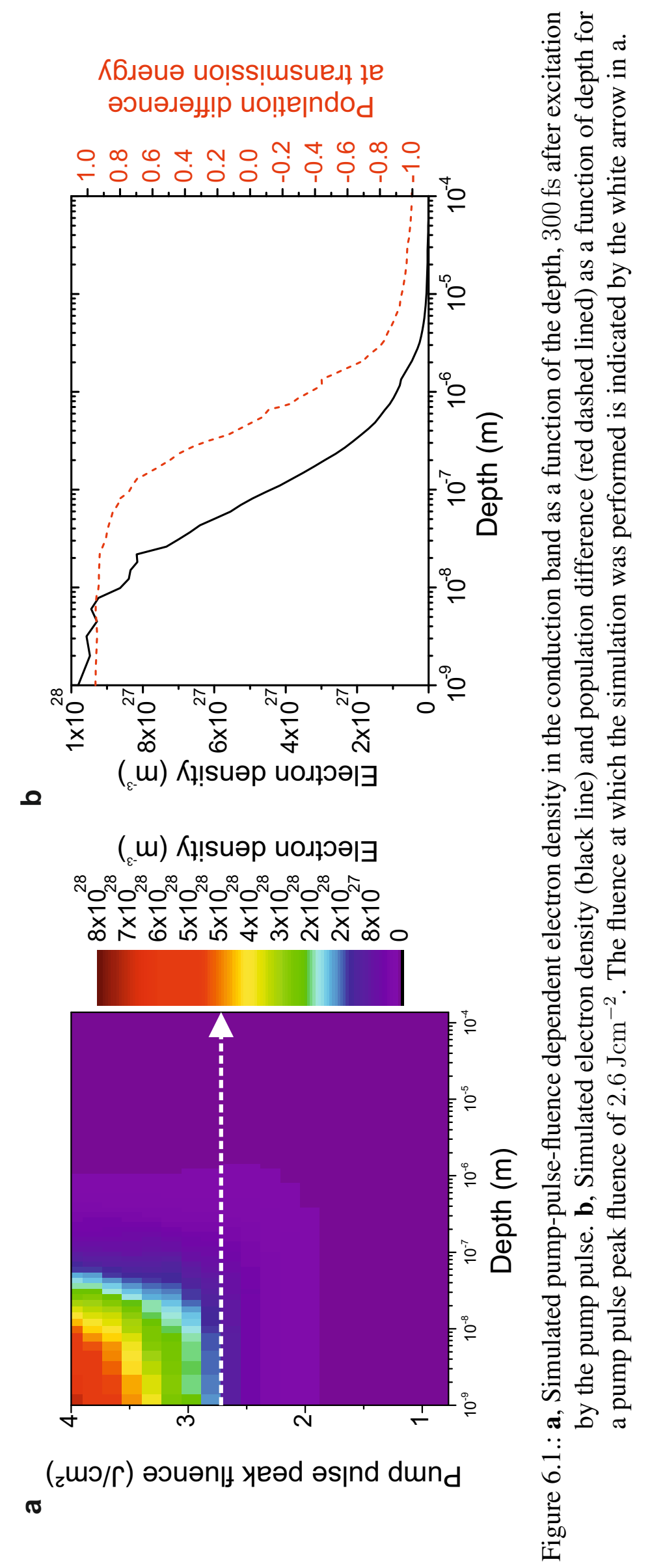


a

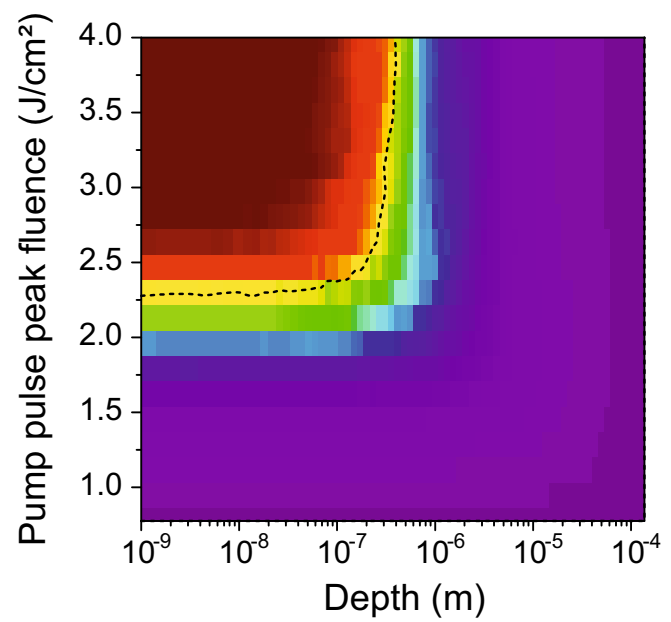

b

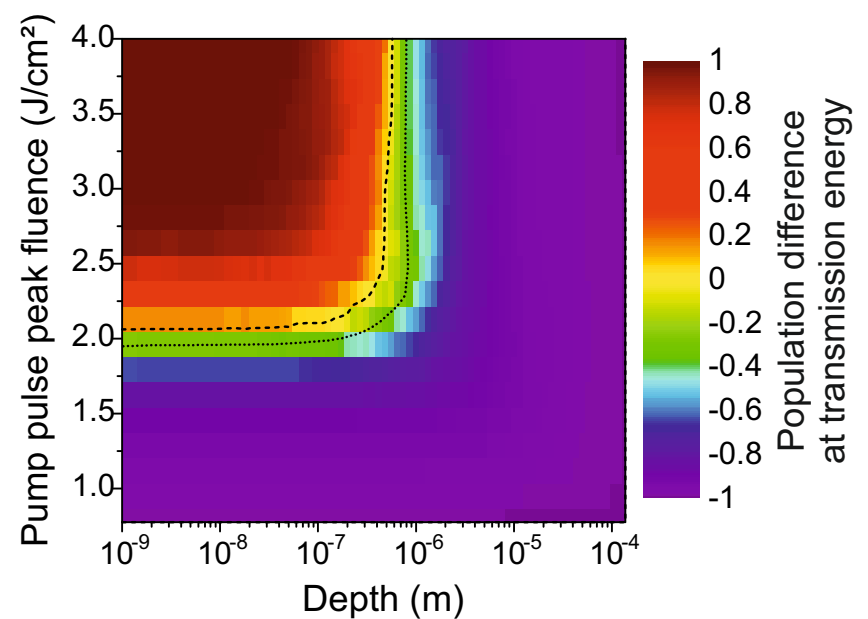

Figure 6.2.: a, Results of the thermalization calculations for an estimated band gap of $9 \mathrm{eV} . \mathrm{b}$, Results of the thermalization calculations for an estimated band-gap of $6 \mathrm{eV}$. The dotted line indicates the region where the carrier-density-dependent band gap shrinkage allows two-photon transitions (see 10). The dashed lines in a and b indicate where the difference function is 0 , i.e. the border between nonlinear absorption and emission.

pump-pulse fluence or conduction-band-electron density. But, depending on how far the pumppulse propagated into the material and was absorbed, its temporal shape might be quite different. Thus, even if the incident fluence or carrier density might occur several times at different depths for different pump fluences, the kinetic energy might deviate. Therefore, it is required to solve the above mentioned problem for each set pump fluence and depth (or delay time, if wanted).

\subsection{Population inversion in excited dielectrics}

From the obtained temperatures and chemical potentials, it is possible to calculate the energydependent distribution of electrons and holes. The reason why we need these distributions is discussed in part II] where the coherent amplification of an ultrashort $400 \mathrm{~nm}$ pulse in an excited sapphire sample is observed and suggested to be based on stimulated multi-photon emission.

In a "classical" laser medium, the requirement for stimulated emission is population inversion between the lower and upper laser transition level. This means that more electrons are located in the upper than in the lower state (for an electronic transition). Usually realized in a three- or fourlevel system, it is a more or less easy task to bring the active medium in the population inversion state [16].

A dielectric material like sapphire, with its valence and conduction band, would in general be considered as a two level system, making population inversion (i.e. more electrons in the $\mathrm{CB}$ than in the VB) an impossible task, as the required fluences would break the scale of the achievable. Even at the ablation threshold of many materials it is assumed that only a few percent of electrons were promoted into the CB [44,70]. As an example, in sapphire this value is assumed to be five percent [70]. However, as we have seen above, valence and conduction band are not two discrete 
levels that are separated by the band gap energy and therefore the electrons and holes are distributed over a broad range of energies.

To account for the energetic distribution of electrons and holes, we want to introduce the term "energetically localized population inversion", that describes the population inversion between the bottom of the conduction and top of the valence band, at the transition energy (wavelength range) of a desired absorption/stimulated emission process.

We calculate the difference of electron densities in the conduction and valence band at the transition energy $\left(E_{\mathrm{T}}\right)$, which is simply given by the difference of the Fermi distributions $(\Delta f)[76]$ :

$$
\Delta f=f_{e, \mathrm{CB}}\left(E_{T}\right)-f_{e, \mathrm{VB}}\left(E_{T}-E_{G}\right),
$$

where $E_{G}$ is the assumed band gap energy and $f_{e, \mathrm{CB}}\left(E_{T}-E_{G}\right)$ the Fermi distribution of electrons in the valence band that can be obtained from the calculated hole distribution (equation 6.6) by:

$$
f_{e, \mathrm{VB}}\left(E_{T}-E_{G}\right)=\left(1-\left(f_{h, \mathrm{VB}}\left(E_{T}-E_{G}\right)\right) .\right.
$$

If the band gap is smaller than the transition energy of the nonlinear absorption or emission process, we average over all possible transitions. If for example, a band gap energy of $9 \mathrm{eV}$ is assumed, it would imply a three-photon process for our probe pulse with a central wavelength of $402 \mathrm{~nm}$ $\left(3 \hbar \omega_{402 \mathrm{~nm}}=9.25 \mathrm{eV}\right)$. Therefore, a possible transition can happen from 0 to $250 \mathrm{meV}$ from the band gap edge. Similarly, when a band gap of $6 \mathrm{eV}$ is assumed, the transition window for a twophoton process is about $170 \mathrm{meV}$. If, for a more appropriate result, the whole spectrum of the probe pulse would be accounted for, the possible transition window would increase accordingly.

In figure 6.2 $\mathrm{a}$ and $\mathrm{b}$ results of the difference function (equation 6.11) described above are shown as a function of applied pump pulse fluence and depth. In figure 6.2 a a fixed band-gap energy of $9 \mathrm{eV}$ and in figure $6.2 \mathrm{p}, 6 \mathrm{eV}$ is used.

In both cases, we observe that the difference function at the surface rapidly increases from -1 (nonlinear absorption) to 1 (population inversion) with increasing pump pulse fluence. The dashed lines in 6.2 a and $\mathrm{b}$ represents when $\Delta f=0$, indicating the transition from nonlinear stimulated emission $(\Delta f>0)$ to nonlinear absorption $(\Delta f<0)$. With increasing depth, the population difference quickly decreases. Overall, we observe that for the higher band gap of $9 \mathrm{eV}$, the required fluences to obtain population inversion are much higher compared to the $6 \mathrm{eV}$ case and it also decreases over a shallower depth. This is attributed to the fact that less energy and a lower CB electron density is required in order to obtain population inversion.

Please note that the excitation modeling (see section 5.2) was performed for a fixed band gap of $9.9 \mathrm{eV}$. In section 10 we will discuss the amplification mechanism in detail, however, we expect that carrier-dependent band gap shrinkage to play a crucial role. For such a shrinkage, neither quantitative nor qualitative descriptions are currently available. This prohibits a dynamic calculation of the pump-pulse-fluence and depth-dependent band-gap shrinkage, thermalization and the population difference. Therefore, we opted to calculate the thermalization process for two fixed band-gap energies.

As an example, in figure 6.1 a the outcome of the extended MRE model and b, this section, is summarized. 6.1 a shows the conduction-band-electron density $300 \mathrm{fs}$ after excitation in dependence on depth and pump fluence. 6.1p shows the depth-dependent electron density (black line) and population difference (red dashed line) for a fixed pump fluence of $2.6 \mathrm{Jcm}^{-2}$. 



\section{Experimental techniques and the extraction of physical quantities}

In this chapter, the experimental setup for the pump-probe as well as the reference-pump-probe setup are described. The measurement and evaluation programs, as well as the corresponding documentation can be found on the group server in the projects folder.

\subsection{Pump-probe setup}

In figure 7.1 a the pump-probe setup is shown. Laser pulses of 30-35 fs FWHM duration at a central wavelength of 785-800 $\mathrm{nm}$ are provided by an amplified Ti-sapphire laser system (Femtolasers Femtopower Pro). The fundamental beam is split into a pump and probe arm by a 50 percent beamsplitter (Femtolasers 50 percent beamsplitter).

The probe beam that passes a high precision motorized delay stage (PI Micos) is frequency doubled in an $200 \mu \mathrm{m}$ thick $\beta$-BBO crystal (Eksma-Optics) and pre-compressed in a prism compressor (Quarzglass). We made use of a nonlinear photodiode ${ }^{1}$ (Silicon carbide, Roithner-Laser) to get a nonlinear signal being proportional to the incident intensity $S \propto I^{2}$. Prior to every experiment the pulse was compressed in this way. Afterwards the energy of each single probe-pulse can be measured by a $3 \mathrm{eV}$ photodiode (GaAsP, Hamamatsu G1117), using a reflex from a beamsplitter or OD filter glass.

The pump beam passes a motorized half-waveplate (B.Halle Nachfl. Gmbh, Achromat 600$1200 \mathrm{~nm}$ ) and is let into our liquid-crystal-modulator-based home-built pulse shaper [18,77], which is used for temporal pulse shaping and dispersion compensation of the pump pulse (see chapter 1). Here we made use of the same nonlinear photodiode we used to compress the SHG pulse. However, for the $800 \mathrm{~nm}$ pulse, the signal is proportional to $I^{3}$.

By using a motorized lambda-half plate (B.Halle Nachfl. Gmbh, Achromat 600-1200 nm) and a thin film polarizer (Codix Color Pro Vis. CW02) inside the pulse shaper we adjust the pump-pulse energy, measured by a photodiode-PD1 (Silicon photodiode) in front of a dichroic recombination mirror (DM).

The pump and probe beams are recombined by a dichroic mirror and focused by a long-workingdistance microscope objective (Mitutoyo M Plan APO $2 \times$ ) onto the surface of a thin dielectric sample. The sample is moved from shot to shot by a motorized high precision $x-y$-stage (PIMicos). After the sample, the probe pulse is imaged by a $40 \times$ magnifying system onto the entrance slit of an imaging spectrometer (Princeton Instruments 320 spectrometer with a cooled Roper CCD array). The imaging system consists of another long-working-distance microscope objective

\footnotetext{
${ }^{1} 2$ photons were required to overcome the bandgap at $400 \mathrm{~nm}$.
} 


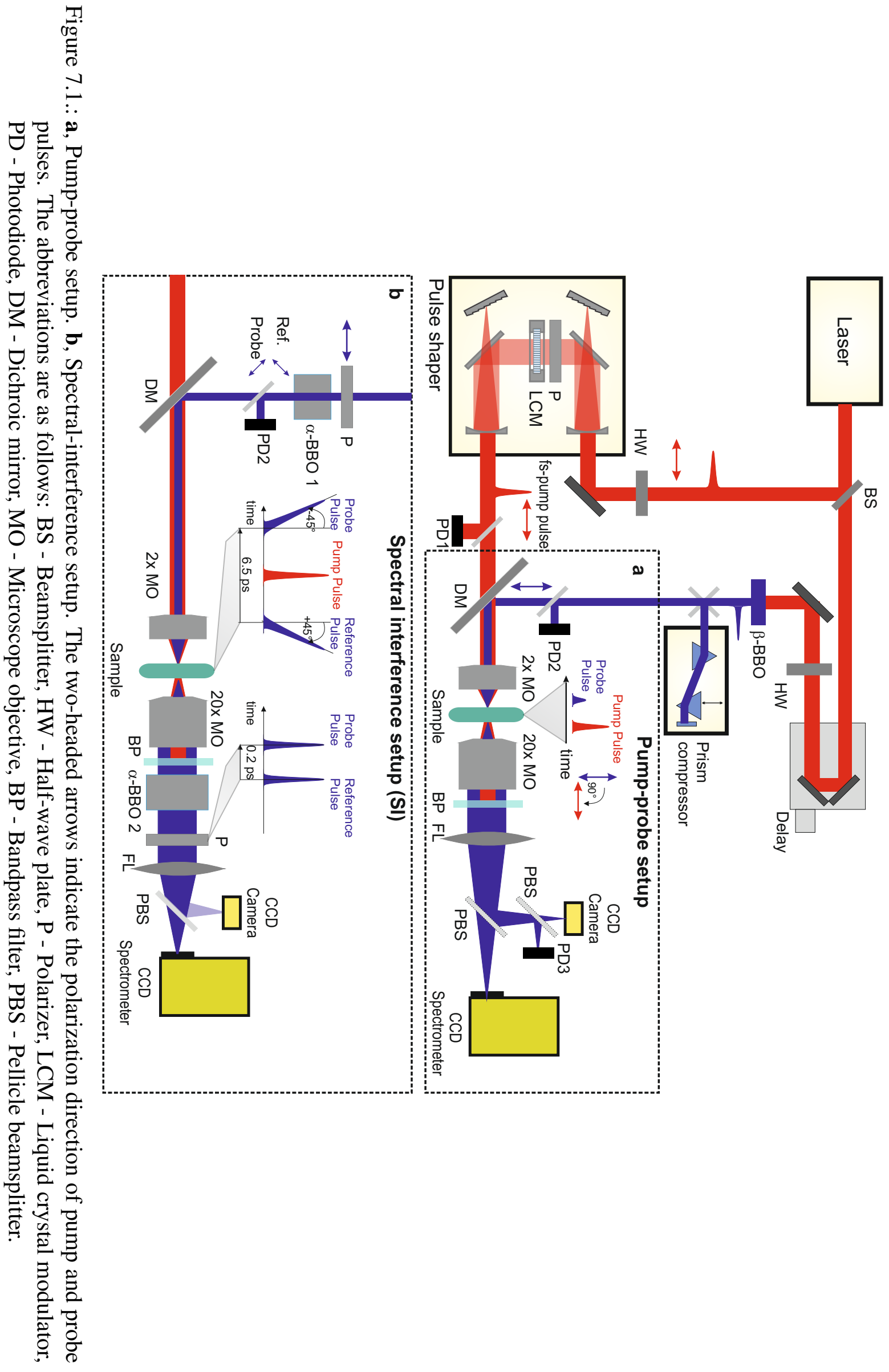


(Mitutoyo M Plan APO 20×) and a $400 \mathrm{~mm}$ focusing lens (Quarz). To block the fundamental pump pulse and possibly any generated white light, we use two $400 \mathrm{~nm} \pm 25 \mathrm{~nm}$ OD4 $2^{2}$ bandpass filters ${ }^{3}$ (BP in 7.1). The spectrometer has 1024 pixels for spectral and 128 pixels for spatial recording, selected by the entrance slit with an adjustable slit size. We used a grating of $1800 \mathrm{~mm}^{-1}$ leading to a dispersion on the CCD camera of $0.033 \mathrm{~nm} / \mathrm{px}$. Therefore, it is possible to measure the spectral properties as a function of the radial position of the spatial beam profile.

The magnification factor $(40 \times)$ of the imaging system was verified by imaging a transmission grating (10 lines per $\mathrm{mm}$ ), that was etched onto a glass slide. For details and evaluation of the data see [78].

After the imaging system, a fraction of the probe beam is sent towards a monochromatic CCD camera (Lumenera Lu135M), as well as a $3 \mathrm{eV}$ photodiode (PD3 in 7.1a) (GaAsP, Hamamatsu G1117). We used pellicle beamsplitters (Thorlabs BP208 uncoated) to avoid ghost-images on the CCD camera. Ghost images are replica of an image caused by internal reflection inside a thick beamsplitter.

The energies of the incident pump and probe pulses are measured in single shot at the indicated positions (PD1 and PD2 in 7.1). We performed measurements in which the photodiodes are calibrated against a commercial calibrated photodiode (Ophir Nova II Powermeter) in the interaction area. Afterwards we performed a calibration scan without a pump pulse for photodiode PD3.

Both beam profiles are characterized on each measurement day in the focal region by imaging the beams onto a beam profiler (Data Ray WinCam) at the position of the entrance slit of the spectrometer.

The temporal profile of the pulses is obtained, prior to each measurement, via different techniques: The pump pulse duration is usually measured with a FROG/Grenoulli device [79]. The probe pulse duration is then measured by the optical Kerr effect as described in Sarpe et al. [11] and shown in the appendix in section 16.1 .2 .

\subsection{Common-path spectral interferometry setup}

This version of the experimental setup (figure $7.1 \mathrm{~b}$ ) is slightly different compared to a typical pump-probe experiment. While the pump-pulse path is equivalent to the one described above, the probe pulse path strongly differs:

After the pre-compression within the prism compressor, the horizontally-polarized $400 \mathrm{~nm}$ pulse is split into a pair of pulses by a $12.5 \mathrm{~mm}$ thick $\alpha$-BBO crystal, having an orientation that is rotated $45 \mathrm{deg}$ with respect to the incident probe pulse, as indicated in figure 7.2. Due to the birefringence of the crystal, the incident pulse is split into two separate pulses, propagating in the ordinary (o) and extraordinary (e) axis of the crystal. As the refractive index of each axis is different $\left(n_{\mathrm{o}}=1.6963\right.$ and $n_{\mathrm{e}}=1.5493$ ), the e- and o- pulse propagate with different velocities, leading to a temporal distance of $6.5 \mathrm{ps}$ when exiting the crystal. The polarization of the probe pulses is shifted by $90 \mathrm{deg}$ with respect to each pulse and $\pm 45 \mathrm{deg}$ with respect to the pump pulse, as sketched in figure 7.2 .

\footnotetext{
${ }^{2}$ This means a 99.9 percent absorption for all other wavelengths.

${ }^{3}$ Edmund Optics bandpass interference filter(400 $\left.\mathrm{nm} \pm 25 \mathrm{~nm}\right)$.
} 


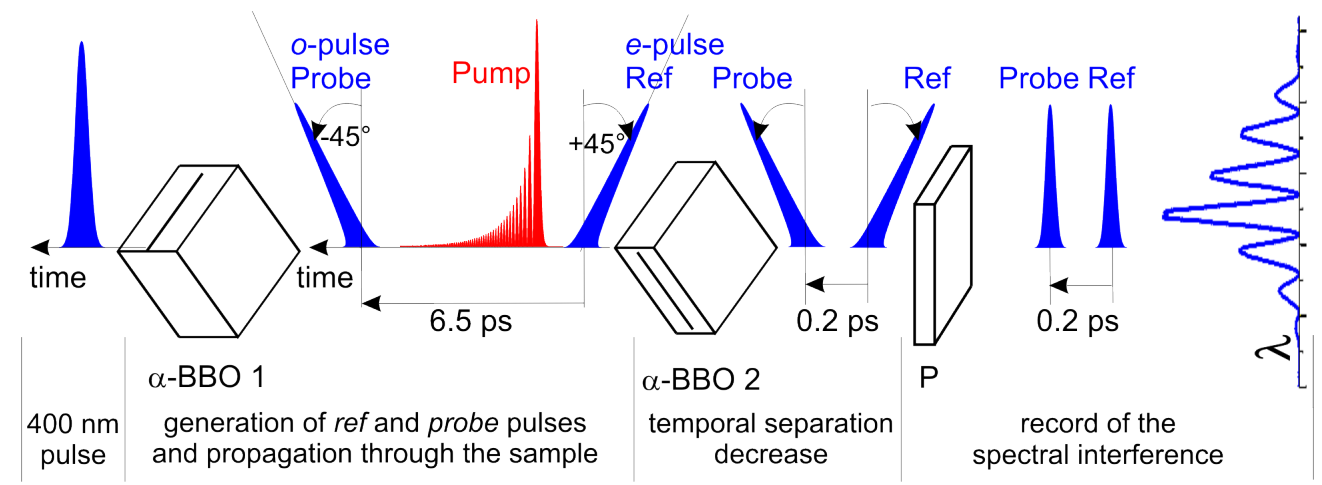

Figure 7.2.: Schematic representation of the probe pulse path. The frequency doubled $400 \mathrm{~nm}$ is, in an $\alpha$-BBO (1) birefringent crystal, split into two orthogonally polarized pulses with a temporal separation of $6.5 \mathrm{ps}$. The two pulses propagates through the sample together with the pump pulse to probe the changed optical properties. A second $\alpha$-BBO (2) crystal is used to reduce the temporal delay before the polarisation is equalized by a polarizer $(\mathrm{P})$. Picture taken from [11].

The pulse that propagated through the crystal on the extraordinary-axis, exiting the crystal first, is called reference pulse, while the other one is named probe pulse.

Then reference and probe pulse are recombined with the pump pulse and focused onto the sample and imaged by the imaging system. Here, after the pump pulse was filtered out by the bandpass filter, we made use of a second $\alpha$-BBO crystal to reduce the temporal distance of reference and probe pulse. This is necessary, as our spectrometer cannot resolve the interference of two pulses that have such a large temporal distance. The second BBO crystal is rotated such that the reference pulse is now propagating on the slow axis whereas the probe pulse propagated through the fast axis, effectively reducing the temporal delay to $200 \mathrm{fs}$, due to a crystal-thickness of $12.2 \mathrm{~mm}$. As the polarization of the two pulses is still $\pm 45 \mathrm{deg}$, we use a polarizer, that is set to a vertical polarization, as shown in figure 7.2 .

Then the two pulses enter the imaging spectrometer and interfere with each other.

Please note than in contrast to the pump-probe setup, the pulse energy of the probe pulse is not measured during the experiment. However, the total probe energy, consisting of probe- and reference pulse, can be measured prior to conducting the measurement.

\subsubsection{Water-Jet}

In part III we describe the investigation of laser excitation in water. We used a water jet, which is created by letting water flow though the thin opening of a polished stainless steel nozzle. The thickness of the water jet has been measured by recording the spectral interference pattern of the reflected pump beam from the front and back surface of the water jet [78] to be approx. $96 \mu \mathrm{m}$. To ensure a fresh sample of water for every pump pulse (approx. every second in single shot operations), a flowing speed of $3 \mathrm{~cm} / \mathrm{s}$ is selected. For our measurements we used bi- distilled water, which can be assumed to be free of any impurities [28]. 

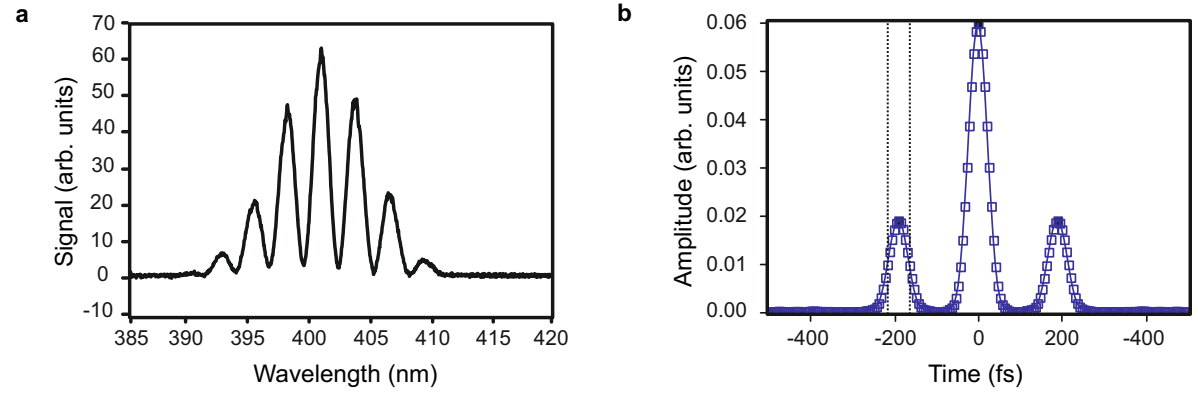

Figure 7.3.: a, Recorded spectral interference signal (no excitation). b, Fourier transformation of the recorded interference signal. The dashed horizontal lines represent the set time window in which the peak for the probe pulse is selected.

\subsection{Extraction of physical quantities}

The reference pulse propagates through the unexcited sample and its electric field is given by (eq. 4.1):

$$
E(z, t)=E_{0}(t) \exp \left(i\left(n_{0} \frac{\omega_{1} z}{c}-\omega_{1} t\right)\right)
$$

where $E_{0}(t)$ is the Gaussian envelope of the electric field that is propagating along the $z$-axis in the time $t . \omega_{1}$ the frequency of the pulse, $n_{0}$ the linear refractive index and $c$ the speed of light in vacuum.

On the other hand, the probe pulse will propagate through the sample after it has been excited by the pump pulse and thus its electric field is quite different and can be written -under the assumption of a constant excitation along the sample thickness- as:

$$
E(z, t)=E_{0}(t) \exp \left(-\frac{\kappa \omega_{1} z}{c}\right) \exp \left(i \frac{k \omega_{1} z}{c}\right) \exp \left(-i \omega_{1} t\right)
$$

Here the electric field is attenuated by the imaginary part $\kappa$ of the complex refractive index $\tilde{n}=k+$ $i \kappa$ (see equation 4.11). It can be seen that in the case that the material is not excited homogeneously along the propagation direction $z, \tilde{n}$ and thus $k$ and $\kappa$ will change as a function of depth making the above written equation for the electric field no longer valid.

However, under the assumption of a slowly varying field in space and a weak gradient in the conduction band density, we can make use of the WKB-solution for an wave propagating in an inhomogeneous plasma [80] providing an expression for the accumulated phase shift of the probe pulse:

$$
\Delta \Phi=\frac{\omega_{1}}{c} \int_{0}^{L} k(z) \mathrm{d} z
$$

where $L$ is the thickness of the sample. Please note that here $k$, the real part of the refractive index is a function of depth $z$. An excitation of the material will therefore lead to a negative phase shift, due to an decreasing real part of the dielectric function (see equation 4.11) and therefore a higher speed of light inside the material.

In the same manner, we can make use of the WKB approximation to obtain an expression for the change in transmission through the sample. We define the transmission $T$ as the ratio of the 
intensity of the probe pulse with the reference pulse. We recall the definition of the cycle-averaged laser intensity:

$$
I(z, t)=\frac{1}{2} \epsilon_{0} c|E(t, z)|^{2},
$$

where $I_{0}(t)$ is the temporal Gaussian envelope of the laser intensity. When neglecting reflection, the transmission through the excited sample is given by:

$$
T=\exp (O D)=\exp \left(-\frac{2 \omega_{1}}{c} \int_{0}^{L} \kappa(z) \mathrm{d} z\right),
$$

with the optical density $O D$.

With respect to the spectral interference measurements, the electric fields of reference and probe pulse can be written as:

$$
E_{\text {Reference }}=E_{0}(t) \exp \left(-i \omega_{1} t\right)
$$

and

$$
E_{\text {Probe }}=\sqrt{T} E_{0}(t) \exp \left(-i \omega_{1}(t-\Delta \tau)-i \Delta \Phi\right)
$$

where $\Delta \tau$ is the temporal delay between reference and probe pulse when entering the spectrometer. This time depends on the experimental conditions, in our case the pulses have a temporal distance of $200 \mathrm{fs}$ upon entrance into the spectrometer.

The CCD camera in the spectrometer then records, spatially resolved, the spectral interference which resembles a Fourier transformation $(F(\ldots))$ of the pulses:

$$
S(\omega)=F\left(\left(E_{\text {Probe }}+E_{\text {Reference }}\right)^{2}\right)=S_{0}(\omega)[1+T+2 \sqrt{T} \cos (\omega \Delta \tau-\Delta \Phi)],
$$

with the spectrum $S_{0}(\omega)$ and the transmission of the probe pulse $T$. The interference pattern consists of a Gaussian shaped spectrum that is centered at the central frequency of the probe pulse and a fringe pattern with a period of $\frac{2 \pi}{\Delta \tau}$ in case of no excitation. It can now be seen, that the introduced phase shift, caused by a changed refractive index, moves the interference pattern. An example of $S(\omega)$ is given in figure $7.3 \mathrm{~b}$. Please note that the wavelength axis is given for convenience.

To extract transmission and phase shift, a further Fourier transform is required:

$$
\mathrm{F}(S(\omega))=(1+T) G(t)+\sqrt{T} G(t+\Delta \tau) \exp (-i \Delta \Phi)+\sqrt{T} G(t-\Delta \tau) \exp (-i \Delta \Phi),
$$

with $G(t)$ given by:

$$
G(t)=\exp \left(-4 \ln (2)\left(\frac{t}{\Delta t}\right)^{2}\right)
$$

and

$$
G(t \pm \Delta \tau)=\exp \left(-4 \ln (2)\left(\frac{t \pm \Delta \tau}{\Delta t}\right)^{2}\right),
$$

where $\Delta t$ is the FWHM pulse duration of the probe/reference pulse. Thus the Fourier transform of the interference pattern delivers an autocorrelation function with an amplitude of $1+T$ centred around $t=0$ fs and two satellite peaks with a reduced amplitude of $\sqrt{T}$ shifted by $\pm \Delta \tau$. This can be seen in figure $7.3 \mathrm{~b}$, which shows the Fourier transformation of the interference pattern in figure 7.3 . 


\subsubsection{Transmission}

To obtain the transmission $T$ from the three peaks shown in figure $7.3 \mathrm{~b}$, we make use of the amplitude-ratio $x$ of side-peak to the main peak, introduced in equation 7.9 .

$$
x(T)=\frac{\sqrt{T}}{1+\sqrt{T}^{2}},
$$

which gives

$$
T(x)=\frac{1-2 x^{2} \pm \sqrt{1+4 x^{2}}}{2 x^{2}} .
$$

Please note that in the experiments the transmission $T(x)$ is obtained from the measured ratio $x$.

Both solutions for $T(x)$ are shown in figure $7.4 \mathrm{a}$ and $\mathrm{b}$. We can see that in case of an equally strong probe and reference pulse $(T=1)$, the ratio is given by $x=0.5$. However, typically the pulses are never of identical intensity and thus the initial ratio $x$ is less than 0.5 . To still obtain a transmission coefficient that starts at $T=1$, we normalize the measured value in case of excitation to the one in case of no excitation. The careful reader might have noticed that it is not possible to distinguish from $x<0.5$ if the reference or probe pulse has less intensity.

However, we can make use of the knowledge about the processes involved in our experiments. If the target material, like sapphire, is excited by the pump pulse, the transmission has to decrease due to the creation of conduction band electrons (see equation 4.11) and therefore we should observe it in the experiment. If the transmission is increasing with increasing pump power, we can expect that initially the reference pulse was weaker than the probe pulse.

As a side note, from plot $7.4 \mathrm{a}$ and $\mathrm{b}$ it can be seen that when starting with an initially lower probe intensity, the slope is much steeper, therefore changes in transmission cause a more drastic change in the ratio of the peaks, making it more sensitive.

Typically is is not expected that a transmission of $T>1$ will be detected. However, an amplification of the probe pulse is measured in the experiments presented in part II. Thus, we intentionally start with a probe pulse that has much lower intensity than the reference pulse, allowing us to measure an increase in transmission. If the amplification is so strong that the (initially weaker) probe pulse becomes more intense than the reference pulse, it will look like the transmission decreases, due to the decreasing ratio $x$. Here the positive solution of 7.12 has to be used. Thus, the interpretation of transmission measurements by spectral-interference has to be done very carefully. In the appendix, a measurement in which a transmission above one was obtained is shown.

However, it is not usually possible to observe a transmission above 1 in our SI experiments. The reason is that the required probe pulse intensity (see part III) is relatively high and the possible maximum probe pulse fluence is limited due to the creation of a reference/probe pulse pair and several reflections at the crystals used in the experiment. A way to optimize the observed maximum amplification in SI experiments would be to get more probe pulse energy or to optimize the temporal compression of the probe pulse to obtain a shorter pulse duration, as the amplification effect is suggested to be based on two-photon stimulated emission (see part III) and thus proportional to the square of the probe-pulse-intensity. 
a

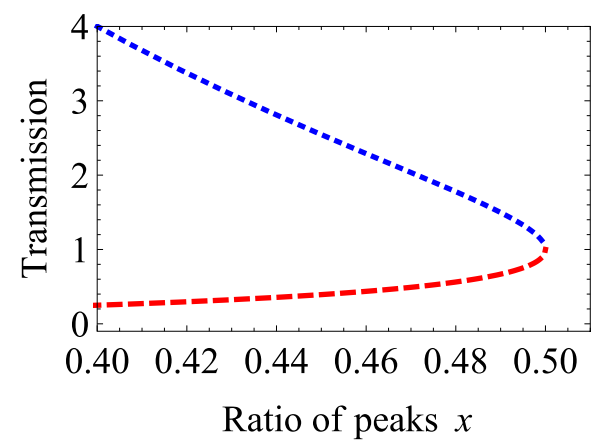

$\mathrm{b}$

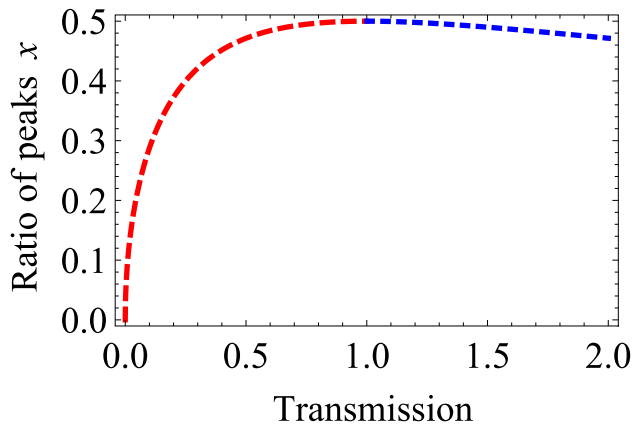

Figure 7.4.: a, Calculated ratio $x$ of side to main peaks from the transmission $T$ and $\mathbf{b}$, vice verse. The red dashed line in a and $b$ represents the negative solution of 7.13 , whereas the blue dotted line is the positive solution.

\section{Transmission in pump-probe}

In the pump-probe experiments, the transmission is measured in three different ways:

- Globally via the calibrated $3 \mathrm{eV}$ photodiode (temporally and spatially integrated)

- Spectrally resolved by the imaging spectrometer (temporally integrated, locally measured)

- Locally by the CCD camera (temporally integrated)

All data is recorded in single shot and no averaging takes place. For each laser shot, the above named quantities as well as the pump pulse energy are measured.

The evaluation of the photodiode measurements is quite simple: The recorded signal is calculated into an energy via a calibration function obtained in a separate calibration. In this measurement, the photodiode that measures the incident probe pulse energy (PD2 in figure 7.1) is calibrated against a commercially available powermeter placed behind the focusing objective. Then, the photodiode that records the transmitted probe pulse energy (PD3) is calibrated against PD2.

The transmitted spectra are usually not transferred into a "Transmission". They are shown as the pure or normalized signals as a function of wavelength.

The evaluation of the CCD images is a little bit more complex: In the first step a background image is taken where no laser light is incident. This image is subtracted from all other recorded images to take into account the background signal of the camera. The second step is the recording of a reference image when only the probe pulse is incident to the sample. To incorporate shot-to-show energy fluctuations of the probe pulse $\mathrm{A}^{4}$ the reference image is multiplied by a correction factor given as the ratio of the probe pulse energy incident (PD2 in figure 7.1) when taking the reference picture (probe only) and the measurement (probe and pump pulse are incident). It is important to apply the correction factor to the reference image and not to the ones from the measurements. The reason is that in the case of nonlinear effects (nonlinear in probe intensity), an application of

\footnotetext{
${ }^{4}$ These fluctuations can be up to 25 percent in the worst case, as the pulse to pulse fluctuations of the amplifier are in the range of 5 percent. As the second harmonic generation is proportional to the intensity squared of the amplifier, we end up with the 25 percent.
} 


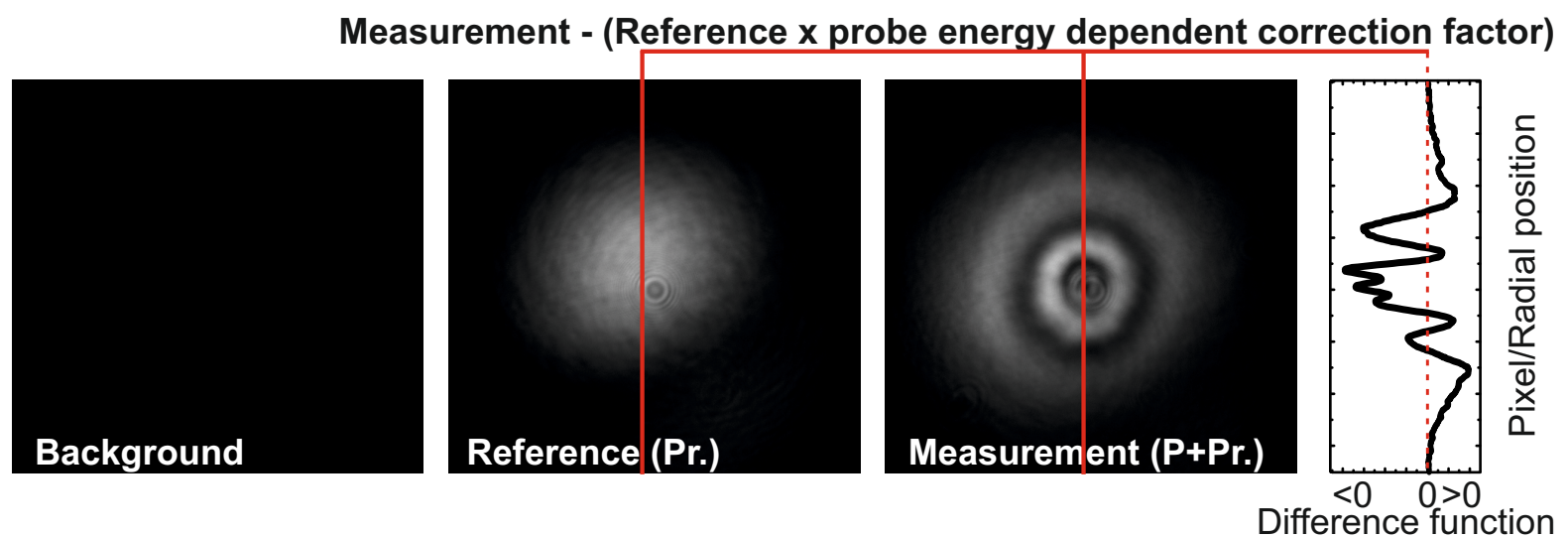

Figure 7.5.: Sketch of obtaining the difference map from the CCD images as described in the text. Pr. is the abbreviation for probe pulse and $\mathbf{P}$. for pump pulse.

a linear correction factor would drastically change the "real" results. To finally obtain a difference map, we simply subtracted the corrected reference image (only probe + correction) from the measurement images (pump + probe).

For a suitable representation, we selected a cut through the probe beam on the CCD camera to obtain a radially-dependent difference signal. This "cut" was chosen to be a window of several pixels in width. All values within this window were averaged to make up for a shot-to-shot movement of the probe beam. This evaluation process is sketched in figure 7.5 .

The local transmission extracted from the CCD measurements is the quotient of a measured image to the corrected reference image at selected positions.

\subsubsection{Phase shift}

In equation 7.9, it can be seen that only the satellite peaks contain the information about the phase shift $\Delta \Phi$. When the side peak is so clearly separated as in our experiment (figure 7.4), the phase shift can be observed directly as a shift of the peak on the time axis. However, a more reliable extraction is by separating a satellite pulse and Fourier transforming it $(\tilde{S}(\omega))$. This will isolate the interference term and the phase as a function of frequency is obtained by taking the complex logarithm of the Fourier transform [81,82]:

$$
\tilde{S}(\omega)=F(\tilde{S(t)})=E_{\text {Probe }} E_{\text {Reference }} \exp \left(i\left(\Phi_{0}-\Phi(\omega)-\omega \tau\right),\right.
$$

where $\Phi_{0}$ is the phase of the reference and probe pulse, $\Phi(\omega)$ the spectral phase and $\omega \tau$ is the phase caused by the temporal delay of probe and reference pulse. The spectral phase is obtained by:

$$
\Phi(\omega)=\Phi_{0}-\omega \tau-\arg \tilde{S}(\omega) .
$$

This measurement will be related to a reference measurement to extract only the shift of the phase introduced by the pump pulse.

$$
\Delta \Phi=\Phi(\omega)_{\text {Measurement }}-\Phi(\omega)_{\text {Reference }}
$$

Thus we obtain the phase shift for each wavelength of the spectrum with a width of around $8 \mathrm{~nm}$. If we assume that the refractive index is constant over this range, we can expect a phase shift tilt 
across the spectrum of only one percent (see equation 7.3). This little effect is ignored and in the final measurements, we average the phase shift of all wavelengths, decreasing noise in the obtained phase shift. If only a single wavelength would be selected, at certain values of the phase shift the amplitude would nearly go to zero increasing the noise or making the phase shift undetectable. 


\section{Part II.}

\section{Laser amplification in excited dielectrics and dynamics of laser-excited dielectrics}





\title{
8. Introduction and outline
}

\begin{abstract}
A S discussed in the introduction, high-band-gap dielectrics are usually transparent for the used probe wavelength of $400 \mathrm{~nm}$. This changes rapidly when these materials are exposed to our ultrashort and highly intense $800 \mathrm{~nm}$ pump pulses. The different interaction mechanisms, introduced in chapter 4, lead to the appearance of an entire catalog of transient nonlinear optical phenomena, like the optical Kerr effect or the metallization of the initially transparent material. Usually, in our experiments, we utilize the changes in the optical properties, i.e. transmission and phase shift to characterize the strength of excitation introduced by the pump pulse due to its interaction with the material, as done in part III. This is an approved straightforward technique used in many experiments. An important rule of thumb that runs right across all publications covering the excitation of transparent materials is that, the harder you pump, the more metal-like the dielectric material becomes [35]. Meaning more and more light is reflected and absorbed due to the increasing amount of electrons in the conduction band (see section 4.1 ).
\end{abstract}

Performing time-resolved spectral interference experiments on sapphire, we expected to observe results that are similar those obtained in other studies [22, 35, 57], which follow this classical rule of thumb.

Our expectations were met only to a certain extent: After the pump pulse passed the sample (section 9.1), the phase shift 7.3 , being a reliable quantity for the indication of the excitation strength, showed a steady increase up to the ablation threshold of the material. The transmission on the other hand behaved quite unexpectedly (section 9.2). Instead of a continuous decrease, we observed an increased for a certain range of pump pulse intensities, eventually becoming larger than 1 , indicating a possible coherent amplification of the probe pulse. These results were reproducible and we made similar observations in measuring the excitation of water in part III

To exclude that this unusual observation was a result of the spectral interference technique (section 7.2), we set up a more simple pump-probe experiment (section 7.1). By directly measuring the transmission of the $400 \mathrm{~nm}$ probe pulse globally via a photodiode, locally with a CCD-camera and spectrally resolved with an imaging spectrometer, we were able to measure the same temporal and energetic dependencies as in the SI experiment and confirm its results. Furthermore, we could show that the probe pulse is not only locally amplified but also globally (9.1.1), meaning that more pulse energy is transmitted than was incident to the sample. This excluded the potential redistribution of light via the propagation of the probe pulse in the highly excited sapphire sample as an explanation and indeed confirmed that the probe pulse is coherently amplified.

Utilizing the imaging spectrometer (section 9.3), we found, either spectral broadening when a pump fluence was used for which absorption was observed or spectral narrowing in case of amplification at higher pump fluences. This was a direct hint for nonlinear interactions (absorption and emission) of the probe pulse with the excited sapphire. This nonlinearity was confirmed by varying the incident probe pulse intensity and measuring all the different quantities (section 9.4). For very low probe intensities we observed no amplification at all, i.e. the transmission simply 
decreased with increasing pump fluence, following the expected behavior, observed in a manifold of studies, as described at the beginning of this chapter.

With increasing probe fluence, the strong absorption of the probe pulse is more and more replaced by coherent amplification, being initially visible as a less strong absorption, followed by local and global gain $(T>1)$. While often the order of nonlinear processes can be extracted from powerlaws of the dependent variable (here: probe-pulse fluence) like in e.g. in classical two-photon fluorescence experiments [83], it is not applicable in this case, as the properties of the excited material drastically change as a function of depth and the measured signals are values integrated along the path of the laser pulse through the sample, discussed in chapter 5 .

The correlation between the laser amplification and the excitation of the sapphire sample, i.e. the promotion of electrons into the conduction band, was further investigated by comparing the excitation dynamics of sapphire versus fused silica (section 9.5). As the conduction band electrons in the latter material only have a typical lifetime in the range of a few hundred femtoseconds, it was the perfect material for a direct comparison. And indeed, we found in fused silica the amplification effect as well, but only as long as the free carriers were present.

Finally, it was possible to identify the origin of the nonlinear amplification to a two-photon stimulated emission process that is discussed and described in detail in chapter 10. Furthermore, by combining an extended multiple rate equation model (sec. 5.2) for the excitation with intrabandthermalization calculations (chapter 6) and a simple propagation model of the probe pulse, it is possible to simulate the amplification process.

As a final test of the proposed amplification mechanism, we made use of temporally asymmetric shaped laser pulses, introduced in chapter 1 . It has been shown that those pulse shapes lead to an excitation in dielectrics that reaches much deeper, compared to ultrashort bandwidth limited laser pulses. Examples for this effect are given e.g. in this thesis (part II] and in [13, 84]. We could show in section (10.2) that by increasing the excitation depth, the local amplification becomes stronger. This is in perfect agreement with our model.

In summary, the time and energy dependence of laser amplification in excited sapphire and fused silica are presented. The results are reproduced by simulations in a qualitative as well as semiquantitative fashion. We showed that a time-delayed ultrashort $400 \mathrm{~nm}$ probe pulse is coherently amplified locally and globally due to the creation of a (energetically-)local population inversion and a carrier-induced band-gap shrinkage following the excitation by an $800 \mathrm{~nm}$ pump pulse.

\subsection{Contributions}

The results presented in this part were obtained in a close collaboration with the group of Prof. Peter Balling from the University of Aarhus - Department of Physics and Astronomy.

\subsubsection{Experimental contributions}

Together with Lasse Haahr-Lillevang, who was a visiting PhD student in the time of January 2015 to March 2015 in Kassel, I finished the extension of the experimental setup to measure solid samples. Lasse helped in particular adapting the measurement software (LabView) and performed, 
together with me, the spectral-interference measurements on sapphire and fused silica. Throughout the project, Cristian Sarpe was supporting the experimental part, by building the photodiodes required for the pump-probe experiments as well with general hints and support. Bastian Zielinski supported this project by reviewing and potentially improving the LabView software for the data acquisition.

\subsubsection{Theoretical contributions}

In the final development phase of the proposed amplification process, (now Dr.) Lasse HaahrLillvang performed extended multiple-rate equation model simulations of the laser excitation of the sapphire sample. This was required as an input for the modeling of the intraband thermalization and resulting propagation and amplification of the probe pulse.

A detailed description of the multiple-rate equation modeling by Lasse Haahr-Lillevang can be found in his $\mathrm{PhD}$ thesis (access via Aarhus University) and [22]. Important input regarding our model, especially the thermalization part, came from Prof. Bärbel Rethfeld, from the University of Kaiserslautern. 



\section{The experiment and experimental results}

The experiment in which the amplification of a $400 \mathrm{~nm}$ femtosecond laser pulse is measured is sketched in figure 9.1. It shows the pump-probe setup (see section 7.1) in which an $800 \mathrm{~nm}$ pump pulse is focused onto the surface of a dielectric sample, i.e. sapphire or fused silica. The subsequent, time-delayed, $400 \mathrm{~nm}$ probe pulse is then incident onto the same position and probes the changed optical properties of the excited sample by being imaged onto a CCD camera, a photodiode and an imaging spectrometer, as explained in detail in the sections 7.1 and 7.2.

In the pure pump-probe setup (see section 7.1) we are able to measure the following quantities:

- Total transmission from probe pulse energy "Global transmission"

- Spatially resolved transmission from the CCD camera "Local transmission"

- Spatially resolved signal difference from the CCD camera "Signal difference"

- Spatially resolved spectral transmission

On the other hand, in the spectral interference setup we can measure (see section 7.2):

- Radially(spatially)-resolved transmission "Local transmission"

- Radially(spatially)-resolved phase shift "Phase shift"

Those observables are highly sensitive to the excitation of the material and therefore to different parameters:

- Varying temporal delay between pump and probe pulse using fixed pump and probe fluence: "Delay scan"

- Varying pump-pulse fluence, while probe fluence and delay time are fixed:

"Pump-fluence scan"

- Varying probe-pulse fluence, while pump fluence and delay time are fixed:

"Probe-fluence scan"

Please note that regarding the delay scan, negative times $t<0$ fs are always referring to a time before the pump pulse is incident to the sample, whereas for positive times, the probe pulse sees the excited sample. Therefore, $t=0 \mathrm{fs}$ refers to the moment of time in which the probe and pump pulse peak intensity overlap in time. 


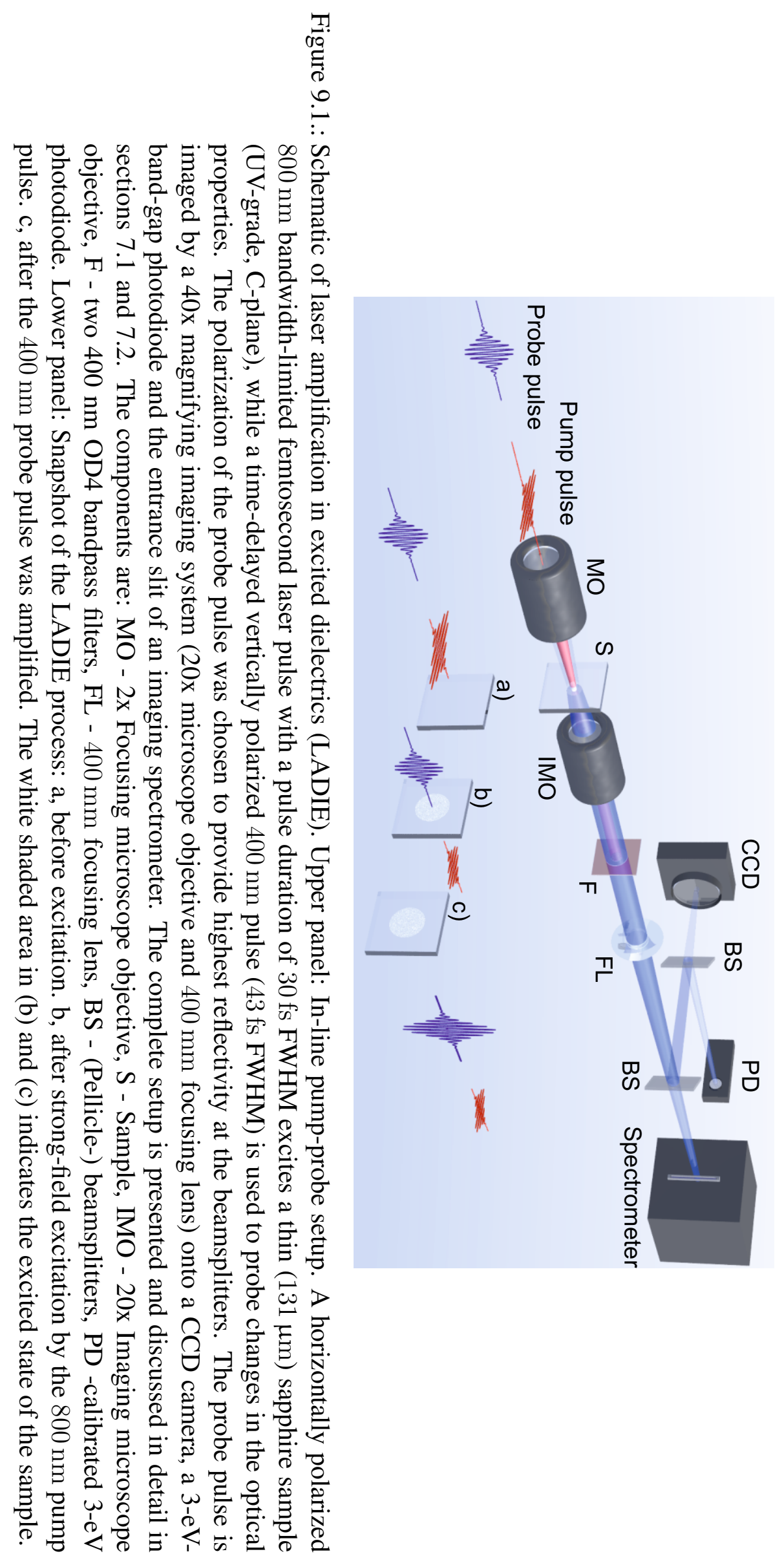




\subsection{Temporal Dynamics of laser excited sapphire and fused silica}

\subsubsection{Global and local dynamics in sapphire}

Figure 9.2 a shows the global transmission of the probe pulse (blue squares) measured with a calibrated $3 \mathrm{eV}$ band-gap photodiode as a function of the pump-probe delay time. For negative times, before the pump pulse arrived, no change in transmission is observed. As the delay time approaches zero, the global transmission of the $400 \mathrm{~nm}$ probe pulse increases to nearly two for a short time during the temporal overlap of pump and probe pulse. During the overlap, nonlinear interactions based on the optical Kerr effect (sec. 4.4) cause cross-phase modulation [3, 4] and probably parametric amplification [85]. 100 to $200 \mathrm{fs}$ after the temporal overlap, the global transmission increases again up to 1.5, providing a direct experimental demonstration of a global gain for the probe pulse as it passes through the laser-excited sapphire under the given experimental conditions.

This behavior is also reflected in the CCD images of the probe pulse in figure $9.2 \mathrm{p}$, where the images show different local contributions: In figure $9.2 \mathrm{p}$ (I) the unperturbed probe pulse after the sample is imaged, whereas in figure 9.2 b(II) probe and pump pulses overlap temporally. $100 \mathrm{fs}$ after the pump pulse (figure 9.2 $\mathrm{p}$ (III)), the central part shows absorption, while for longer delay times a bright inner ring is observed (figure $9.2 \mathrm{~b}(\mathrm{IV})$ ). Please note that the applied pump fluence of $2.7 \mathrm{Jcm}^{-2}$ is below the measured visible ablation threshold $\left(3.2 \mathrm{~J} / \mathrm{cm}^{2}\right)$ of sapphire. We define -ablation- as the permanent material modification visible in optical transmission microscopy (i.e. in a 40x magnified image of the glass sample).

The local transmission within the bright ring in figure $9.2 \mathrm{~b}$ (IV) reaches a factor of 1.4. In the temporal evolution (red circles in figure 9.2 $\mathrm{k}$ ), this local transmission experiences a strong decrease during the overlap between pump- and probe pulses, as reported in a manifold of studies [12,44, 62]. Shortly after the pump pulse, the probe exhibits very low transmission followed by a slow increase. The maximum local amplification is reached at around $300 \mathrm{fs}$.

To quantify these spatial features as a function of the pump-probe delay time, we take the difference between the excited and the unexcited sample along a horizontal line through the beam as described in section 7.3.1: Figure 9.2 shows the difference signal in dependence on the radial position $r$ and the pump-probe delay time. The spatial dimensions of the pump- (red line) and probe pulses (blue line) are indicated by the Gaussians along the left axis. At the temporal overlap ( $t=0 \mathrm{fs}$ ), a negative difference at the centre $(r=0 \mu \mathrm{m})$ is visible, which we interpreted as absorption [12]. At large radii, the difference is positive which is also seen as a bright outer ring in the CCD image (figure $9.2 \mathrm{~b}(\mathrm{II})$ ). This behavior is expected to result from nonlinear propagation during the temporal overlap of the pulses based on the Kerr-lens effect (see section 4.5).

At later times ( $>100 \mathrm{fs}$ ), the negative difference in the center persists, being attributed to freecarrier absorption by the $\mathrm{CB}$ electrons created by the pump pulse through strong-field excitation 3.1. This strong absorption is visible as a dark area in the center of the probe pulse in figure 9.2 (III). We note that in addition, a positive difference at larger radii around $12-20 \mu \mathrm{m}$ remains. These features are observed as soon as CB electrons were generated and thus we relate them to signatures of plasma scattering and defocusing of the probe pulse (see section 4.5). This effect might 


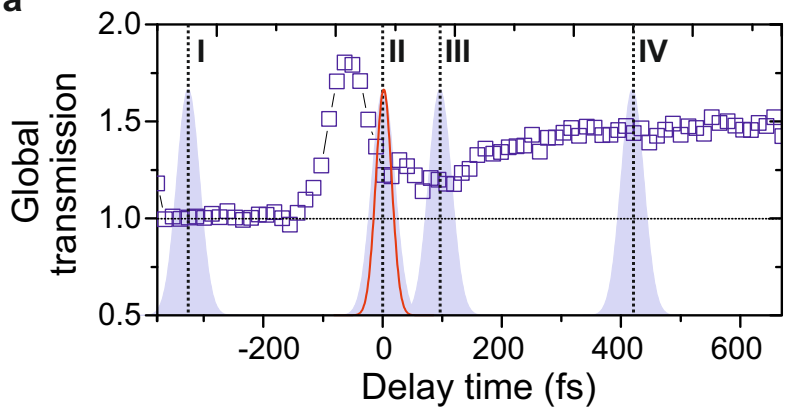

C

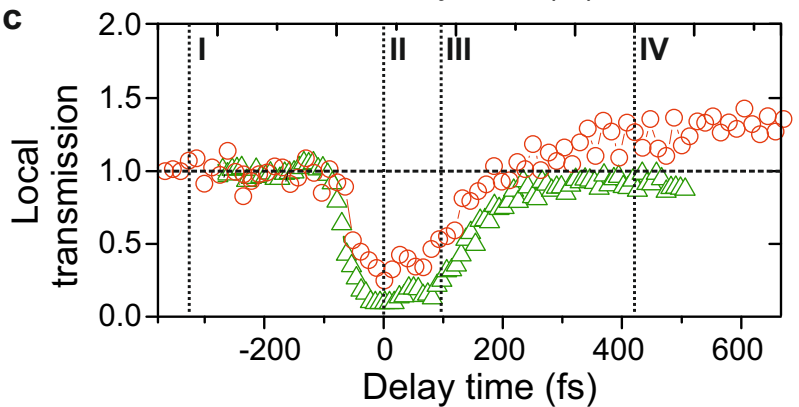

d

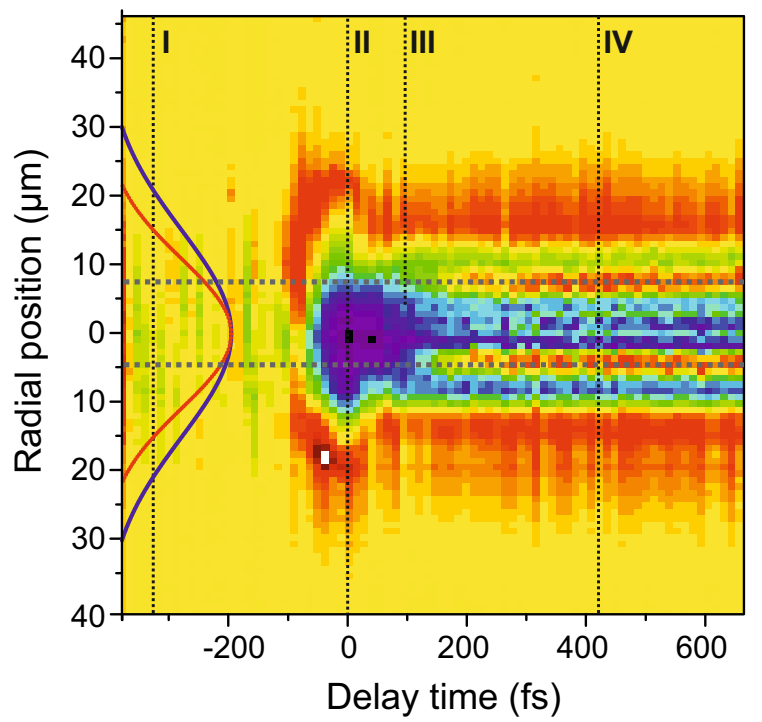

b

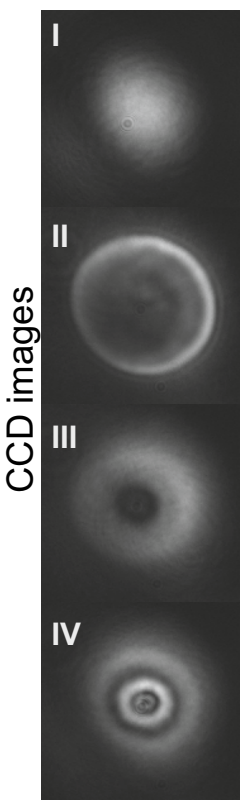

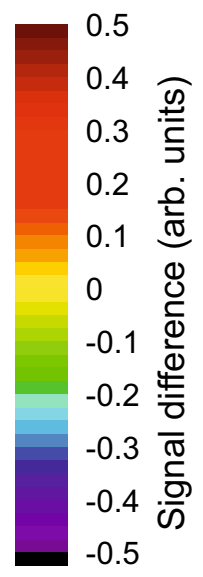

Figure 9.2.: a, Spatially integrated transmission of the probe pulse. b, CCD images of the probe pulse. Vertical dashed lines and Roman numbers in (a, c, d) represent the times at which CCD images in (b) were taken. The red-solid Gaussian in (a) centered around time zero sketches the pump pulse (30 fs FWHM). The temporal delay between pump and probe pulse at certain values (Roman numbers) is visualized by the blue-shaded Gaussian shaped areas of 43 fs FWHM. c, Pump-probe delay time dependent local transmission extracted at the positions indicated by the horizontal grey dashed lines from the CCD images (red circles) and measured by spectral interference (green triangles), extracted from figure 9.7. c, 2D difference plot of the cross section of the CCD images for a pumped and an unexcited sample as a function of delay time. Red and blue lines represent the spatially Gaussian beam profiles of pump and probe beams. The experimental parameters were: Probe-pulse peak fluence: $40 \times$ $10^{-3} \mathrm{Jcm}^{-2}$, pump-pulse peak fluence: $2.6 \mathrm{Jcm}^{-2}$. 


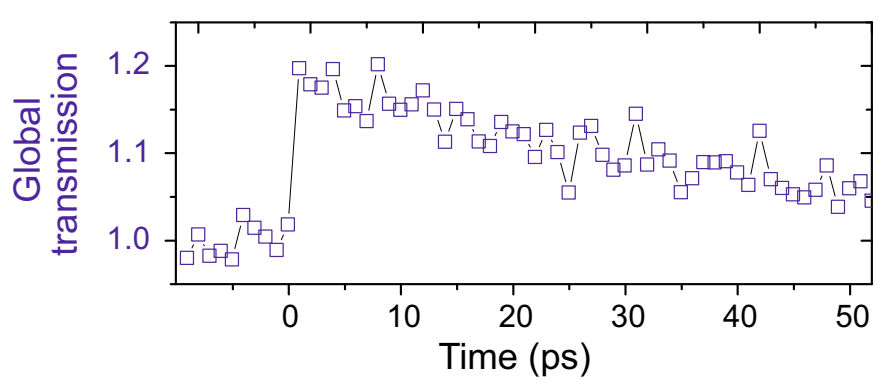

Figure 9.3.: Pump-probe delay-time dependent global transmission in sapphire for an applied pump fluence of $2 \mathrm{Jcm}^{-2}$ and a probe fluence of $40 \times 10^{-3} \mathrm{Jcm}^{-2}$.

contribute to a reduced transmission at positions of small radii, as light might get redistributed outwards.

At even longer delay times, two lines of positive difference located in the range of $r=5$ to $7 \mu \mathrm{m}$ appear, which we relate to being signatures of the local amplification seen in figure $9.2 \mathrm{~b}$ (IV) as the bright inner ring. In figure $9.2 c$ we compare the temporal evolution of this local transmission (red circles) to results of the spectral-interference measurement (green triangles), which is only sensitive to coherent emission is shown (see section 7.2). We observe identical dynamics, proving the coherence of the amplification process.

Please note that in the light appearing at larger radii a slight increases in the local transmission on the same timescale as the amplification is observed, supporting the idea that this light underwent redistribution from radii closer to the center.

\section{Long delay scan in sapphire}

While it takes the amplification around $300 \mathrm{fs}$ to reach its maximum, it is visible locally and globally for more than $50 \mathrm{ps}$ as demonstrated in figure 9.3 . It shows the pump-probe delay-time dependent measurement of the global transmission up to $50 \mathrm{ps}$. We also observe that the transmission starts to decrease after several picoseconds. We attribute this decrease to the recombination of $\mathrm{CB}$ electrons back into the $\mathrm{VB}$ (see section 3.4), which is usually occurring on this time-scale [45,75, 86].

The reason why the global transmission is slightly lower (1.2 instead of 1.5) than in the short delay scan can be found in the applied pump pulse fluence, which is in this case lower. This is further discussed in the next section. 


\subsection{Pump pulse fluences dependence in sapphire}

In the last section we saw that the probe pulse shows different spatial features after propagation through the excited sapphire sample. This can directly be related to the local fluence of the $800 \mathrm{~nm}$ pump pulse.

In figure 9.4a CCD images of the probe pulse are shown for increasing pump-pulse peak fluence. We used a fixed pump-probe delay time of $500 \mathrm{fs}$, at which pump and probe pulses are clearly separated in time. At low pump fluence, we observe no changes in the beam profile of the probe pulse (figure 9.4(i)). A higher value leads to absorption, seen as the black spot in figure 9.4 (ii). Increasing the pump fluence to $2.2 \mathrm{Jcm}^{-2}$ leads to the appearance of local amplification, seen as the bright spot in figure 9.4 (iii). Note that around the amplification area, absorption is still visible as a dark ring. Further increase of the pump fluence allows the amplification to move to larger radii, whereas in the centre the transmission decreases (figure 9.4a(iv)). As the amplification area follows the local fluence distribution of the pump pulse, we look now at the local dependencies.

In figure $9.4 \mathrm{~b}-\mathrm{c}$ the radially-resolved pump-fluence-dependent phase shift and transmission values are shown. The data is obtained via spectral-interference (SI) of the probe beam with a reference beam, as described in section 7.2. This provides the most sensitive way of measuring the coherent changes in transmission. We used a probe-beam diameter that exceeds the pump beam by more than 2.5 times [11,12], assuring that the local probe fluence is nearly constant over the excited area. The influence of the ratio of the spot sizes to the measured values is discussed in the appendix in section 16.3 .1

The phase shift represents the change in the real part of the refractive index during and after the excitation (see section 7.2). An increase in the real part of the refractive index results in a positive phase shift, similar to the optical Kerr effect (see chapter 4). A decrease of the refractive index leads to a negative phase shift as for example caused by conduction band electrons [12, 22, 57]. For a low pump fluences, no change in the phase shift is visible. However, as soon as the threshold for strong-field excitation is reached at about $0.8 \mathrm{Jcm}^{-2}$, a further increase in fluence leads to a negative phase shift, as seen in figure $9.4 \mathrm{p}$. The curve for the phase shift along the centre line (figure $9.4 \mathrm{~d}$ ) reaches its minimum of around $-6 \mathrm{rad}$ at a peak fluence of approx. $3 \mathrm{Jcm}^{-2}$ close to the measured visible ablation threshold at $3.2 \mathrm{Jcm}^{-2}$ in our experiments. Higher pump fluences lead to a slightly increased (i.e. less negative) phase shift, which has been observed for water, shown in part [II] [11, 12], and sapphire [22,66] as well.

We observe that the phase-shift values follow the spatial pump-fluence distribution of a Gaussian beam up to the maximum change of the phase shift, as seen in figure $9.4 \mathrm{p}$. For higher fluences, the radial distribution of the phase ceases to follow the local fluence. We expect self-induced propagation effects [87, 88] of the pump pulse to be negligible below this threshold [12]. This is elucidated further in the appendix (see section 16.3). Note, that the onset of amplification is observed well below this pump fluence.

The radially-resolved transmission data in figure 9.4k, obtained by SI, shows that regions of absorption and local amplification also follow the spatial pump-fluence distribution up to $3 \mathrm{Jcm}^{-2}$, indicating that the amplification effect is directly linked to a certain local fluence and thereby to a certain level of excitation (phase shift). Therefore, depending on the incident pump-pulse fluence, the local amplification is either located in the centre of the pump pulse, as shown in figure 9.4a(iii), or at higher radii, as a ring-like shape (figure 9.4a(iv)). 


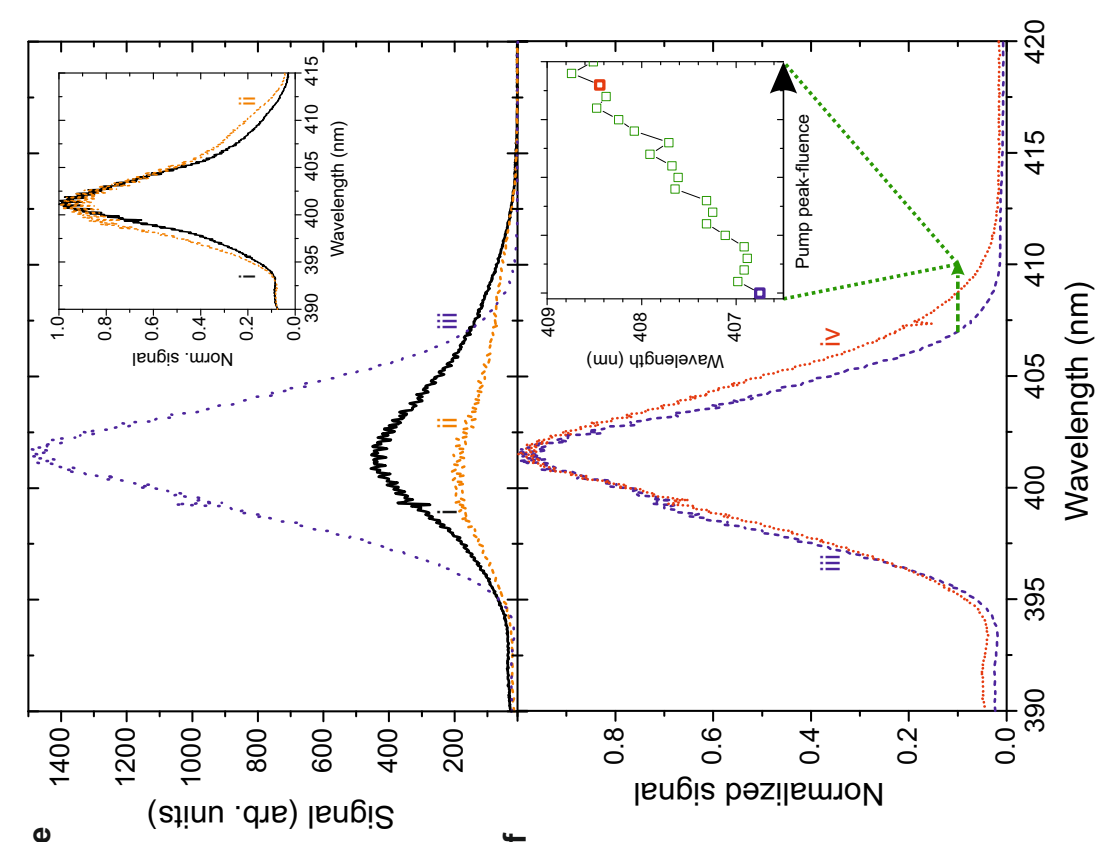

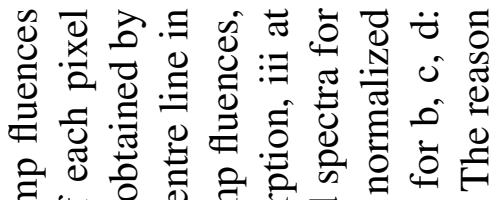

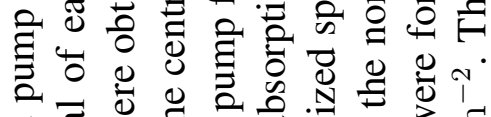
글 啳.

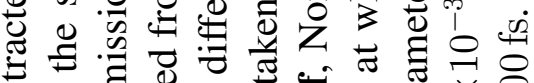
ن $\dot{0}$ 它

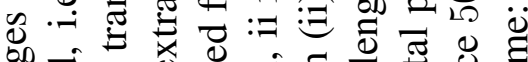

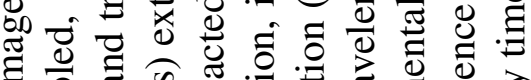

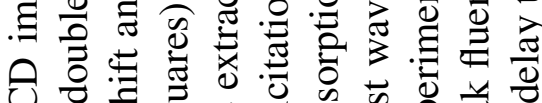

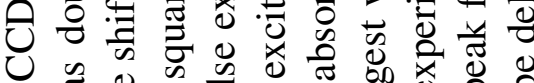

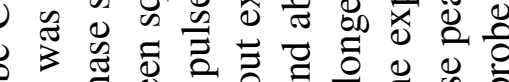

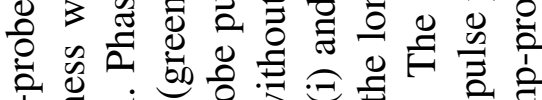

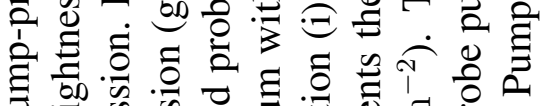

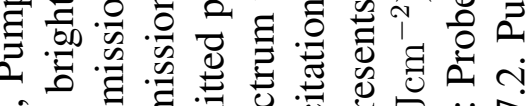

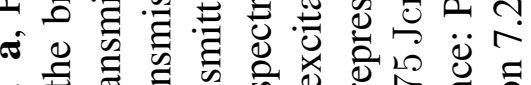

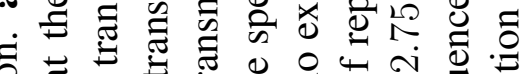

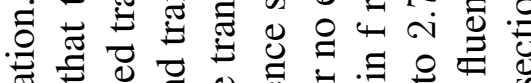
. ब त्ञ

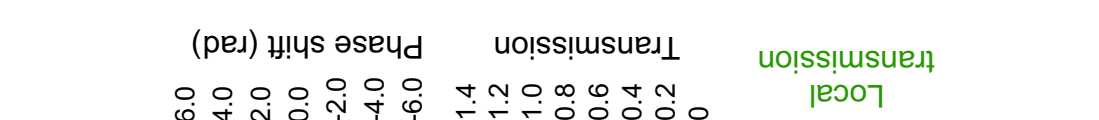

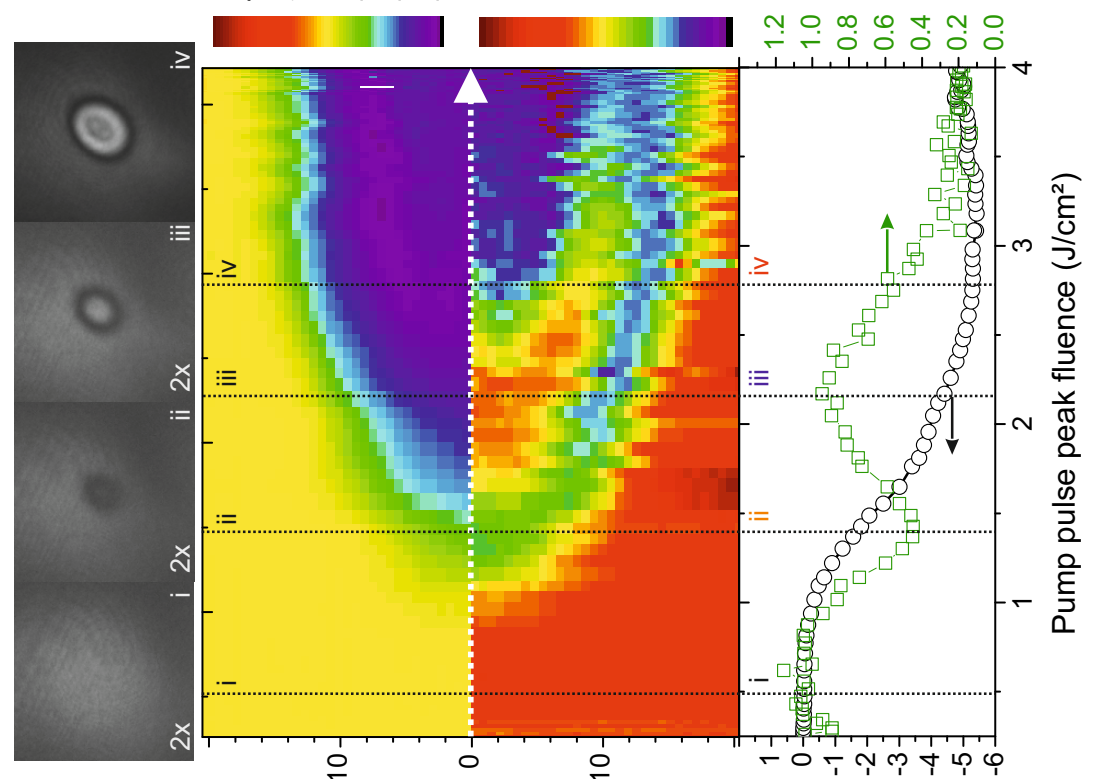

(url) uo!!!!sod je!pey

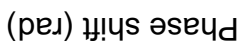

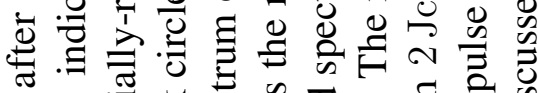

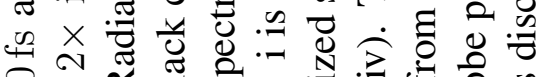

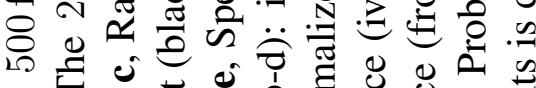

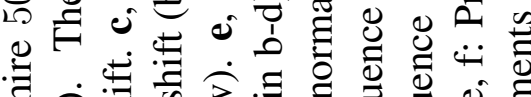

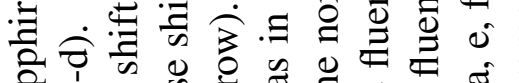
芯

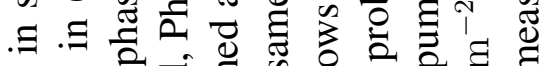

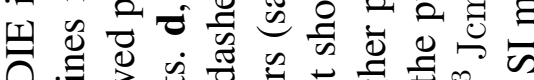

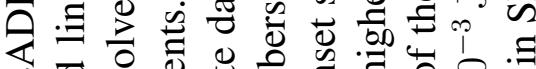

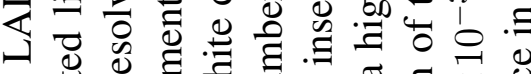

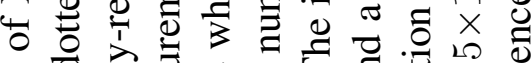
ช

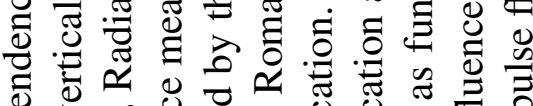
లँ

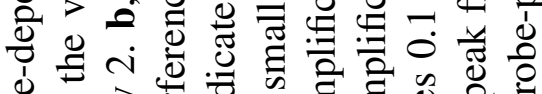

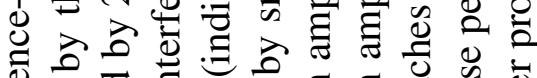

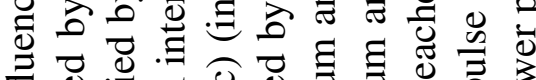

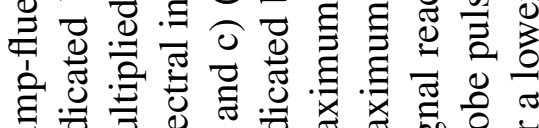

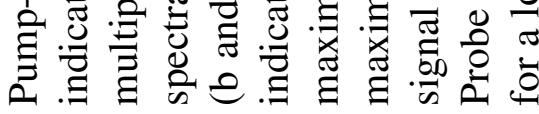
$\ddot{+}$ 苛

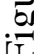


In figure $9.4 \mathrm{~d}$, the local transmission in the center of the pump pulse (green squares) is shown. It decreases with increasing pump fluence, as it was observed in a manifold of studies on laser-excited dielectrics [11, 12] and is seen as the dark area in figure 9.4 (ii). However, at around $1.5 \mathrm{Jcm}^{-2}$ peak fluence, it starts to increase to nearly one again before it finally drops.

\subsection{Spectral properties of excitation in sapphire}

The coherent nature of the measured local amplification in SI is a strong indication for stimulated emission. In such a process, we expect certain features or changes in the spectrum of the amplified probe pulse. In figure 9.4 we present the selected measured spectra of probe pulses that propagated through the sample that was either unexcited (black solid line), excited to strong absorption (orange dashed line) or to strong amplification (blue dotted line). The spectra were taken at a single radial position $r=0 \mu \mathrm{m}$ in the pump-probe configuration (see section 7.1) and at the fluences indicated in $9.4 \mathrm{~b}-\mathrm{d}$ by the lower Roman numbers (i - iv).

In case of absorption (figure 9.4e(ii)), nearly all parts of the probe spectrum are absorbed; only in the wavelength regime above $413 \mathrm{~nm}$, the absorption is slightly less strong.

In the case of amplification (figure 9.4(iii)) at higher pump fluence, we observe that the central wavelength of $402 \mathrm{~nm}$ is strongly amplified by almost a factor of 3.7 for the given experimental conditions. One might wonder why a transmission factor of 3.7 is obtained, whereas in the pure pump probe scheme it was "only" 1.4 under similar excitation and probing conditions. The reason is that $T=3.7$ is the transmission of the central wavelength of the probe pulse, whereas in pumpprobe and SI experiments, the whole spectrum is taken into account. If we integrate the spectra in 9.4 , we observe that the transmission of around 1.8 is significant lower than the value for the central wavelength, showing again that not all parts of the spectrum are amplified equally or are even absorbed.

The inset in figure 9.4 displays the normalized spectra for the no excitation (i) in comparison to strong absorption (ii). We observe a slight spectral broadening, especially in the red wing in case of absorption, which we attribute to an additional absorption process of nonlinear order. Additional evidence for this nonlinear absorption process is presented in the next section.

Figure 9.4f shows the normalized spectra in case of maximum amplification (iii) and for a higher pump power (iv). With increasing pump fluence, the narrow amplification spectrum broadens towards longer wavelengths. The inset emphasizes this by showing the longest wavelength at a 10 percent level of spectral intensity as a function of the applied pump fluence. By increasing the peak fluence of the pump pulse from 2 to $2.75 \mathrm{Jcm}^{-2}$, this long-wavelength limit increases by approximately $2 \mathrm{~nm}$. The fact that the amplified spectrum is significantly narrower than the spectrum in the case of no excitation, points to an amplification process of nonlinear order [16].

\subsection{Probe pulse fluence dependence in sapphire}

The best way to clarify hints towards a nonlinear amplification process is to investigate the dependence of the transmission on the probe-pulse fluence. In the following pump-probe measurements, a fixed delay time of $500 \mathrm{fs}$ and a pump fluence of $2 \mathrm{Jcm}^{-2}$ was used. 
a

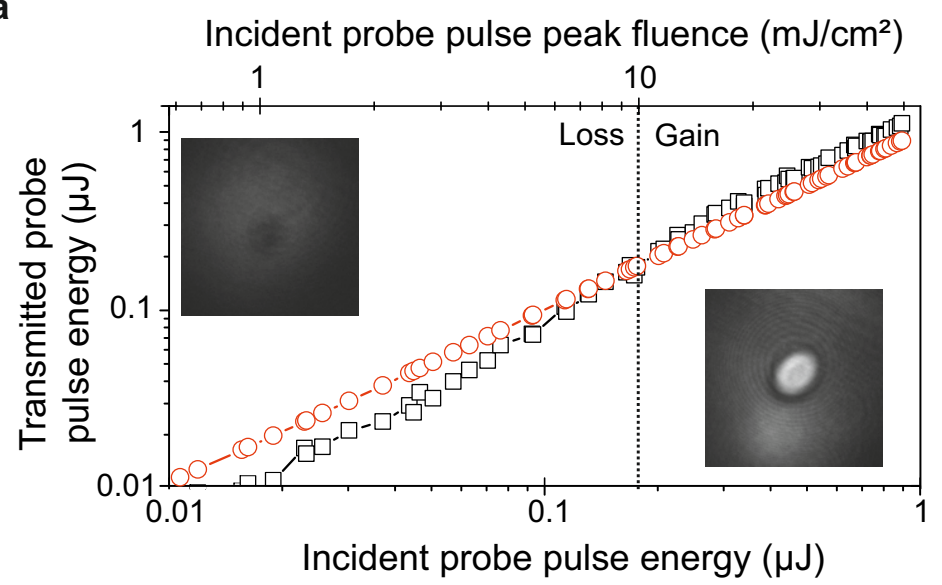

b

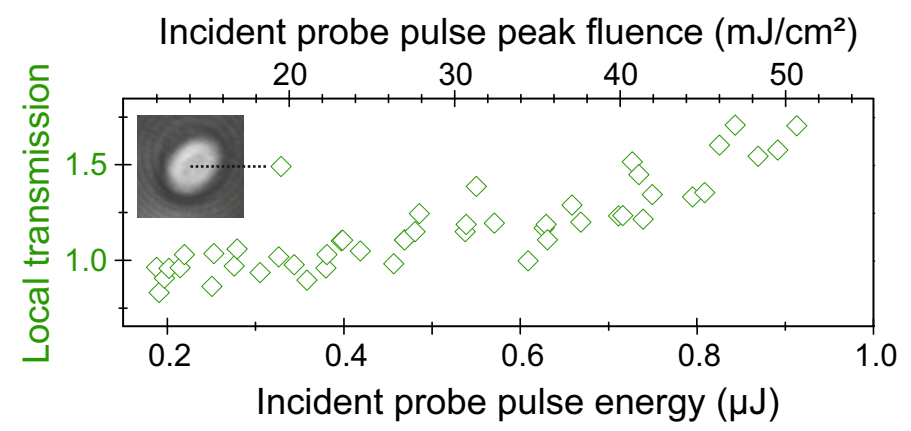

Figure 9.5.: Probe-fluence-dependence of LADIE in excited sapphire $500 \mathrm{fs}$ after excitation. a, Spatially integrated transmitted probe pulse energy in a double-logarithmic representation for an unpumped (red circles) and excited sapphire sample (black squares). Insets in a: Exemplary CCD images of a transmitted probe pulse using very low (left) and very high probe-pulse-energy after transmission. b, Green diamonds show the local transmission in dependence on the incident probe pulse energy and fluence for an amplification region, as indicated on the inset. For convenience, the scale for the probe-pulse peak fluence is provided on the top axis of the graph. The experimental parameters were: Pump-pulse peak fluence: $2 \mathrm{Jcm}^{-2}$. Pump-probe delay time: $500 \mathrm{fs}$. 


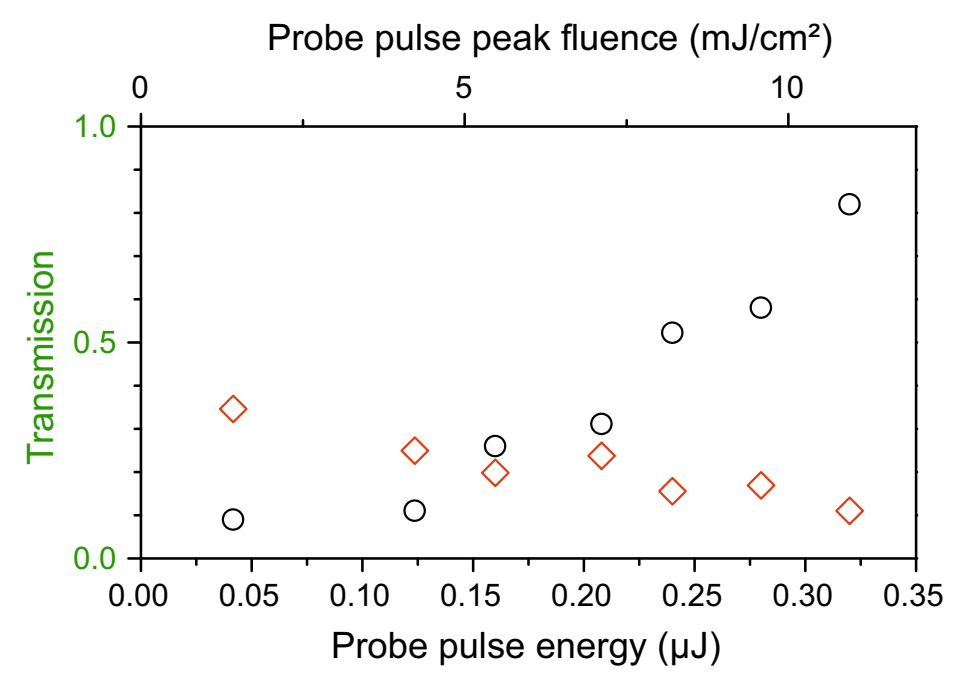

Figure 9.6.: Probe-pulse energy dependence in excited sapphire in SI measurements. Local transmission at a local pump fluence of $1.4 \mathrm{Jcm}^{-2}$ (red diamonds) and $2.2 \mathrm{Jcm}^{-2}$ (black circles) in dependence on probe-pulse energy. For convenience, the peak fluence of the probe pulse is given on top of the graph. The pump-probe delay time was $500 \mathrm{fs}$.

\subsubsection{Global behavior}

In terms of a spatially-averaged, global transmission, we observe that at low probe-pulse fluence the transmission through the excited sample (red circles in 9.5 ) is smaller than in the unexcited case (black squares), generally agreed upon by us and in the literature as being caused by free carrier/inverse-Bremsstrahlung absorption [22,57]. For a fluence above $0.16 \mathrm{Jcm}^{-2}$, global gain can be obtained under the given experimental conditions, which is a strong indication that the amplification effect is not caused by light redistribution due to plasma defocusing or diffraction. Also, we notice again a strong differences of local amplification (inner part) and local absorption (first black ring) seen in the image of the probe pulse in the right inset in $9.5 \mathrm{a}$. As the global measurement is averaging over these features, we also investigated the local dependencies.

\subsubsection{Local behavior}

In figure $9.5 \mathrm{~b}$, the local probe-energy-dependent transmission of an amplified region (green diamonds), measured with the CCD camera, is shown. The position at which the data was evaluated is indicated by the inset in 9.5 b. Please note that this data and the photodiode measurements (figure 9.5 a) result from the same scan. As the CCD camera has a limited dynamic range, only images for probe energies above $0.18 \mu \mathrm{J}$ were recorded, or at least show more than the background noise. We observe a nonsaturating, nonlinear, increase of the local transmission with the incident probe-pulse energy and peak fluence, respectively. 


\section{Local behavior in spectral-interference}

The local transmission, measured with the CCD camera is not as sensitive as the SI measurements and therefore we performed several pump-fluence dependent SI measurements (as shown in figure 9.4) for different probe pulse energies. In figure 9.6 the transmission at a local pump fluences of $1.4 \mathrm{Jcm}^{-2}$ (red diamonds) and $2.2 \mathrm{Jcm}^{-2}$ (black circles) in dependence of the probe-pulse fluence are shown. At the chosen values of the pump fluence, absorption and amplification is most pronounced for high probe fluence (figures $9.4 \mathrm{~d}$ (ii) and (iii) and 9.6), respectively. We observe that the transmission at a pump fluence of $1.4 \mathrm{Jcm}^{-2}$ actually decreases for increasing probe fluences (red diamonds in figure 9.6). This hints to a nonlinear absorption process possibly made accessible by the excitation of the sample. This is consistent with the hints of a nonlinear absorption seen in the spectrum at similar pump fluence conditions (see figure 9.4).

For the pump fluence of $2.2 \mathrm{Jcm}^{-2}$, at which maximum transmission and amplification in figure $9.4 \mathrm{~d}$ was reached, we observe that for a low probe fluence, nearly no light is transmitted through the sample (black circles in figure 9.6) due to the strong absorption the conduction band electrons. Increasing the probe-pulse fluence leads to a successive nonlinear increase of the transmission, as observed in figure 9.5. It might seem unsatisfactory that the measurement ends here before a transmission above 1 was reached. However, as discussed in section 7.2, the maximum available pulse energy for the probe pulse in the spectral-interference configuration is strongly limited by several reasons. The most significant one is that the initial $400 \mathrm{~nm}$ pulse is split up into reference and probe pulse with an intentionally stronger reference pulse, giving the probe less than 50 percent of the initial energy. An exemplary measurement in which a transmission of above one was obtained is given in the appendix in section 16.1.6.

The full pump-pulse dependent SI measurements from which data in figure 9.6 was extracted are shown in appendix in section 16.1.4.

\subsection{Comparison of global and local dynamics between sapphire and fused silica}

The radially-resolved, pump fluence dependent SI measurements in figure 9.4 showed that the local amplification correlates to a certain phase shift, i.e. a certain CB electron density distribution inside the sapphire sample. To pin down the assumed correlation, we compare time dependent SI measurements of sapphire and fused silica, as the latter provides us with different CB electron dynamics (see chapter 2). Due to the ultra fast formation of defects, CB electrons will be trapped into excitonic states (see section 3.4) located within the band gap of the fused silica, on a timescale of only 150 fs [22, 57, 89].

In figure $9.7 \mathrm{a}$ and $\mathrm{b}$ phase-shift and transmission measurements as functions of the pump-probe delay time in the SI experiment for both materials are shown. We applied a peak fluence of $1.2 \mathrm{Jcm}^{-2}$ for fused silica (figure 9.7b) and 2.2 $\mathrm{Jcm}^{-2}$ for sapphire (figure $9.7 \mathrm{a}$ ). The pump fluences were chosen to provide a high transmission/amplification in the center. The chosen pump pulse fluences are below the respective (visible) ablation thresholds of the materials (fused silica $2.5 \mathrm{Jcm}^{-2}$ and sapphire $3.2 \mathrm{Jcm}^{-2}$ ). Please note that the data shown in the graphs are extracted from a spatiallyresolved measurement, shown in the appendix in figure 16.5 . 


\subsubsection{Sapphire}

\section{Phase shift}

Approaching time zero, the phase shift (black circles in figure 9.7) starts to rise caused by the optical Kerr effect, which increases the refractive index (see 4.4). However, we observe that after the short increase, the phase shift stagnates around a value close to 0 before it rapidly drops to a value of $-3.8 \mathrm{rad}$. The reason for the stagnation is explained easily: For negative times, close to time zero, the probe pulse propagates together with the rising flank of the pump pulse (see the pulse shapes in figure 9.2 ), probing the intensity dependent optical Kerr effect. The closer time zero is approached, the higher the intensity of the pump pulse is and thus it starts to promote a high density of carriers into the conduction band counteracting the optical Kerr effect (see chapter 4). With increasing time, the falling flank of the pump pulse does not have sufficient intensity to counteract the changes caused by the $\mathrm{CB}$ electrons, marking the end of the pump pulse interaction with the sample. This is clarified when this scan is compared to one with a very low pump fluence, being insufficient for the excitation of carriers (see figures 16.3 and 16.4 in the appendix).

Although the phase shift rapidly drops to a value of $-3.8 \mathrm{rad}$, we observe that with increasing delay time, the phase shift further decreases to its lowest value of $-4.2 \mathrm{rad}$ within $200-300 \mathrm{fs}$. We relate this to be a continued avalanche excitation by highly energetic electrons.

\section{Transmission}

The transmission (green squares in figure 9.7a) strongly decreases during the temporal overlap, mostly due to redistribution of light towards larger radii, caused by the OKE. This can be seen in the CCD images in figure 9.2. We observe that after time zero was reached, the transmission starts to slightly increase before it drops again by only a few percent. This small feature could easily be overlooked, but it occurs right at the same time as the phase shift drops to $-3.8 \mathrm{rad}$. Thus, at this time step, the low transmission is dominated by the absorption by $\mathrm{CB}$ electrons and no longer by the OKE. This small feature is also visible in the local time-dependent transmission obtained from the $\mathrm{CCD}$ images in figure 9.2k. For longer delay times, the local transmission increases back to a value of one with a rise time of around $200 \mathrm{fs}$. The time in which the amplification is clearly present, is indicated by the red shaded area. Please note that in both, time- and fluence-dependent measurements (figure 16.5) the enhanced transmission is strongly connected to a phase shift of around -4 rad.

\subsubsection{Fused silica}

\section{Phase shift}

In fused silica, the phase shift (black circles), presented in figure $9.7 \mathrm{p}$, increases due to the OKE just as in the case of sapphire. As soon as the temporal overlap decreases for positive times, the contribution by the $\mathrm{CB}$ electrons is present, causing a negative phase shift of approx. $-4 \mathrm{rad}$ at the given excitation conditions. In contrast to sapphire, this phase shift does not persist on the investigated time scale. Instead, it quickly increases to positive values of $0.8 \mathrm{rad}$ within $150 \mathrm{fs}$. This is caused by the trapping process leading to a high density of excitons (see section 3.4), as 


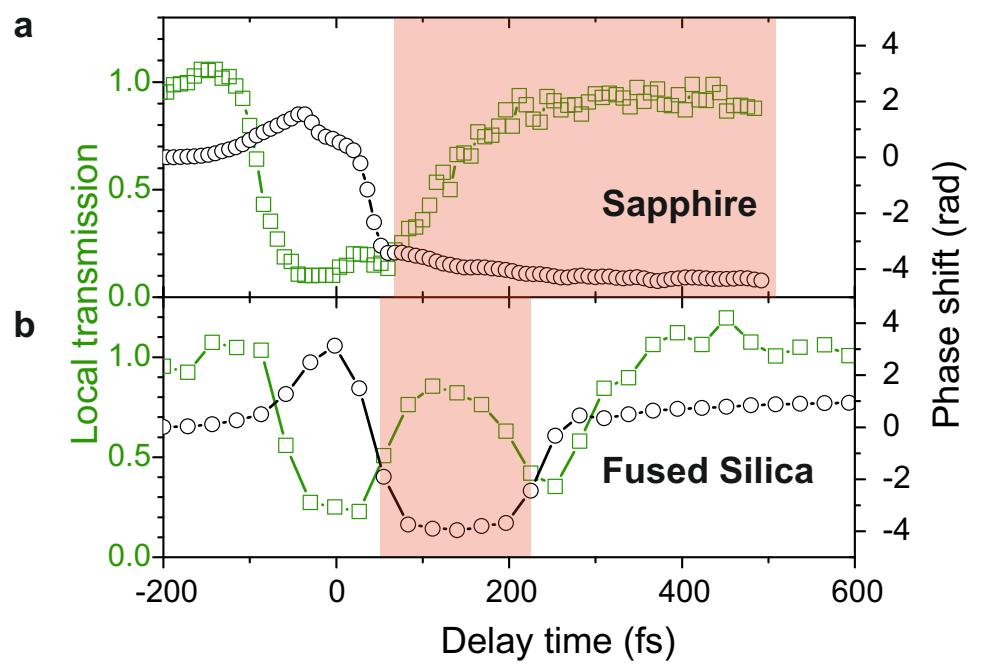

Figure 9.7.: Time dependence of LADIE in sapphire and fused silica. a, Pump-probe delay-time dependent local transmission (green squares) and phase shift (black circles) sapphire. $\mathrm{b}$, the same quantities as in (a) measured for fused silica. Extracted at $r=0 \mu \mathrm{m}$ from figure 16.5. The experimental parameters were in a: Probe pulse peak fluence: $10 \times 10^{-3} \mathrm{Jcm}^{-2}$, pump pulse peak fluence: $2.2 \mathrm{Jcm}^{-2}$. In b: Probe pulse peak fluence: $9 \times 10^{-3} \mathrm{Jcm}^{-2}$, pump pulse peak fluence: $1.2 \mathrm{Jcm}^{-2}$.

they contribute with a positive phase shift (see sec 4.3) to the measurement. Once the electrons are trapped, they will remain in this state until they decay into colour centres (defect states) or recombine back to the valence band on a time scale of a few hundreds of picoseconds [35, 57, 62, 75]. In the radially resolved phase shift data shown in the appendix (figure 16.5k), the local fluence dependence of the phase shift at different times is clearly visible.

\section{Transmission}

The transient local transmission in fused silica also exhibits similarities and differences compared to sapphire: After the initial absorption for pump-probe delay times close to the temporal overlap we see an increase in transmission attributed to the amplification effect, as the high transmission is accompanied by a negative phase shift around $-4 \mathrm{rad}$, which matches the results in sapphire. However, with the continued reduction of CB electrons due to self-trapping around $200 \mathrm{fs}$, free-carrier absorption in the $\mathrm{CB}$ again dominates over the amplification process resulting in the observed drop in the transmission signal. Shortly after the phase shift reaches its characteristic positive value for the STEs ( [22,44]), the transmission relaxes to one because of the emptying of the CB. The transient transmission of the probe pulse in fused silica in combination with the STE dynamics verifies the importance of a high number of CB electrons for LADIE and the presence of similar excitation conditions in both materials during amplification. As STEs are located within the band gap at around $5.2 \mathrm{eV}$ [22,89], they do not absorb single photons from the probe pulse and therefore for long delay times fused silica is transparent again. An interaction of the highly intense probe pulse with the STEs has to be investigated in the future. 


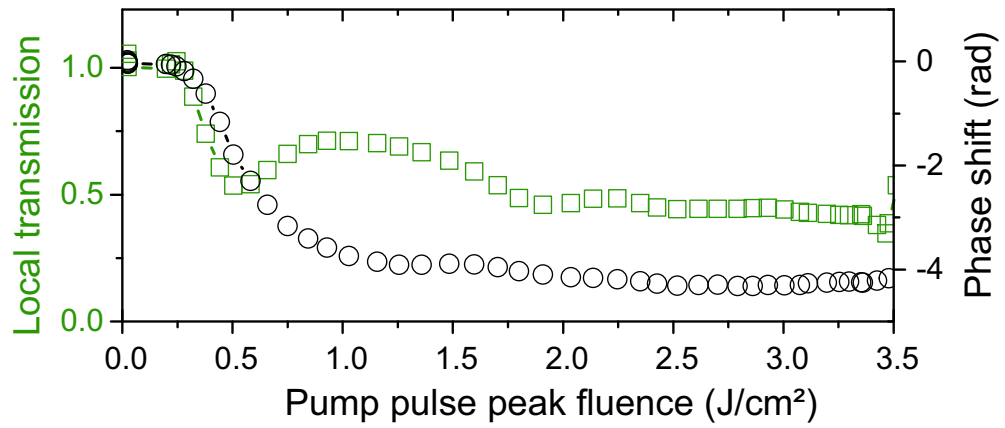

Figure 9.8.: Pump-fluence dependent SI measurement in fused silica. Local phase shift (circles) and transmission (squares) extracted at $r=0 \mu \mathrm{m}$ for fused silica. Experimental parameters were: Pump-probe delay time $180 \mathrm{fs}$. Peak fluence of the probe pulse: $8 \times 10^{-3} \mathrm{Jcm}^{-2}$

\subsubsection{Pump pulse fluence dependence in fused silica}

As seen in the last section, the time dependent measurements in fused silica indicate similar dynamics in the appearance of the amplification process. Here, we investigate the pump-fluence dependent dynamics at a time step, at which the contribution to the optical properties is dominated by the conduction band electrons.

Figure 9.8 shows the pump-pulse fluence dependence of transmission and phase shift in fused silica. This measurement was taken at a fixed delay time of approx. $180 \mathrm{fs}$ (compare figures 9.7 and $16.5 \mathrm{a}-\mathrm{d}$ ). The phase shift (black circles) decreases strongly with increasing pump fluence and reaches its minimum of $-4.2 \mathrm{rad}$ around $2.5 \mathrm{Jcm}^{-2}$, which is close to the measured ablation threshold. For higher pump fluence, the phase shift does not increase as strongly as in the case of sapphire (figure 9.4).

The local transmission (green squares) decreases to around 0.5 and then increases up to a peak fluence of around $1 \mathrm{Jcm}^{-2}$ before it drops again. For high pump fluence the transmission stagnates at a value of around 0.5 , which is higher than for sapphire $(T<0.2)$. The reason for this and a slightly lower increase in transmission $(T=0.71)$ caused by LADIE in comparison to the results obtained in the delay scan in figure $9.5 \mathrm{p}(T=0.85)$ is that the chosen delay time does not exactly coincide with the temporal maximum in figure 9.5. Therefore, complete delay scans at different pump powers are shown in the appendix in section 16.1.3. Both, phase shift and local transmission show dependencies of the amplification on the excitation condition very similar to those obtained in sapphire.

The reason that the required fluence for excitation, measured by the changes in phase shift and transmission, is lower than in the case of sapphire is expected to be a lower band gap of only $9 \mathrm{eV}$ [22], being 10 percent less than what is the expected of sapphire.

Please note the full radially-resolved data set for phase shift and transmission are shown in the appendix in figure 16.11 . 


\section{Proposed amplification mechanism of LADIE and simulations}

The observed amplification in a band-gap material like sapphire or fused silica indicates that conditions for stimulated emission are fulfilled. A simple two-level scheme would not allow those conditions to be fulfilled, as explained in chapter 6 .

Therefore we introduced a three-step-amplification process:

- The first step towards the amplification is the excitation of sapphire by the $800 \mathrm{~nm}$ pump pulse, leading to a non-equilibrium electron distribution in the $\mathrm{CB}$, as sketched in figure 10.11.

- In the second step, the electronic system thermalizes due to electron-electron and hole-hole scattering. Usually within a hundred femtoseconds, after internal thermalization, the CB electrons and holes are in Fermi-like distributions [45, 46, 75], as shown in figure 10.1p and explained in section 3.3 and chapter 6 .

- In the third and final step, the probe pulse interrogates the excited dielectric, where the conditions for amplification (by stimulated emission) depend on the difference of electron density in the $\mathrm{CB}$ and VB at the transition energy $\left(E_{T}=n \hbar \omega_{\text {Probe }}\right)$ as described in detail in section 6.1. Please note that in any excitation condition, free carrier absorption is present and thus a competing process.

As the band gap of sapphire is much larger than the photon energy of the probe pulse, the amplification process must be based on a nonlinear mechanism. The order of such a process $(n)$ is usually determined by the band gap of the material. For UV-grade sapphire typically a value of $E_{\text {Gap }}=9.9 \mathrm{eV}$ [22,70] is used. Therefore, the excitation of sapphire with $800 \mathrm{~nm}$ pulses is usually associated with a six- or seven photon process [22, 62, 70].

To verify the proposed amplification mechanism, we simulated the steps described above. The excitation of the sapphire sample is calculated via an extended multiple rate equation (MRE) model sketched in section 5.2, which provides input for the quasi Fermi-distributions describing the thermalized electrons and holes (see chapter 6). Finally, we use a very simple propagation model taking into account the free-carrier absorption, as well as nonlinear emission or absorption, described by:

$$
\frac{\partial I(t, z)}{\partial z}=-\alpha(N(t, z))+\Delta f(z) \cdot \sigma_{n} \cdot I(t, z)^{n}
$$

While $\alpha(N(t))$ is the CB electron density dependent absorption coefficient described in chapter 5. $\sigma_{n}$ is a nonlinear emission/absorption coefficient ${ }^{1}, \Delta f$ is the difference function indicating if conditions for population inversion is fulfilled, described in section 6.1. Its values range from

\footnotetext{
${ }^{1}$ This is a fitting parameter.
} 

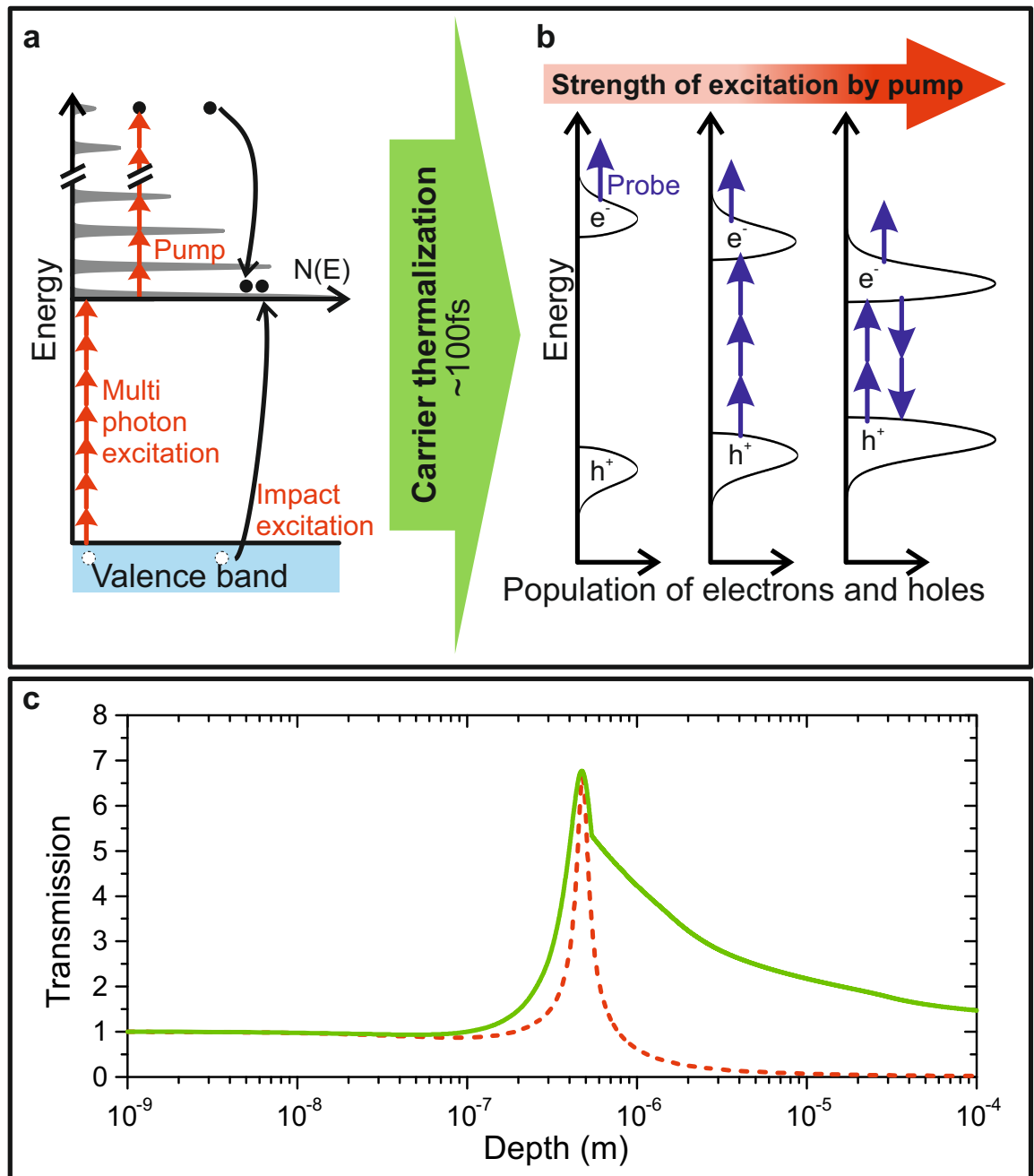

Figure 10.1.: Excitation and amplification mechanism and results of numerical simulations. a, Schematic drawing of the nonlinear excitation process by multi-photon absorption of the $800 \mathrm{~nm}$ pump pulse as well as impact excitation by high-energy electrons. The initial discrete electron distribution $(N(E))$ is sketched in the conduction band (shaded areas). b, Schematics of quasi-Fermi electron and hole distributions after carrier-thermalization in dependence on the strength of excitation, indicating the carrier-dependent band gap shrinkage as well. The blue arrows indicate free-carrieror nonlinear absorption or emission. c, Simulation of the depth-dependent transmission of the probe pulse $(402 \mathrm{~nm})$ in case of a $9 \mathrm{eV}$ band gap and three-photon absorption/emission (red dashed line) and assuming a two-photon process based on carrier-induced band-gap shrinkage (to $6 \mathrm{eV}$ ) (green line). 
-1 (nonlinear absorption) to +1 (population inversion), therefore including both processes in the simulation.

The red-dashed line in figure 10.1 represents the simulated local depth-dependent transmission of the probe pulse, assuming a band gap of sapphire at $9 \mathrm{eV}$ and therefore a nonlinear stimulated emission or absorption for the $402 \mathrm{~nm}$ probe pulse of the third order $(n=3)$. We observe that the transmission increases rapidly with increasing depth due to the self-reinforcing nature of multiphoton stimulated emission. However, at the depth where conditions for stimulated emission are no longer fulfilled (see figure $6.2 \mathrm{a}$ dashed line), nonlinear absorption takes over, causing a dramatic decrease of the transmission in addition to the free carrier absorption by the electrons in the conduction band. The initially amplified light is therefore not able to reach the end of the sample $2^{2}$,

We thus propose that the main reason for a persistent amplification is carrier-induced band-gap shrinkage, which was recently observed in bulk-silicon [76] and 2D dielectric materials [90,91]:

When the sample is unexcited (red circles in figure 9.5 $\mathrm{k}$ ), (nonlinear) absorption of the probe pulse is not observed at all. Consequently, the probe pulse has insufficient intensity to induce a fourphoton absorption processes. Assuming band gap of $9.9 \mathrm{eV}$ and a probe pulse energy of $3.08 \mathrm{eV}$ this would be the lowest order process possible. Only when the material is excited by at least $1.5 \mathrm{Jcm}^{-2}$ pump pulse peak fluence, spectral (figure 9.4) and transmission measurements (figure 9.6) show signs of minor nonlinear absorption of the probe pulse in addition to the free-carrier absorption. This indicates that the band gap is effectively reduced to nearly $9 \mathrm{eV}$ making a threephoton absorption process accessible, as sketched in figure $10.1 \mathrm{p}$. A further increase of the pump fluence will presumably decrease the band gap into the range of $6 \mathrm{eV}$ and simultaneously transfer the material into a population-inversion state, allowing it to act as a two-photon gain medium for the probe light. A further experimental hint of the carrier-dependent band gap shrinkage, besides the obvious amplification, is found in our spectral investigations, where we observe the spectral broadening of the amplified probe pulse, presented in section 9.3 .

Unfortunately, at the current time, there is neither a quantitative nor a qualitative description available for the ultra fast carrier-induced band gap shrinkage in high band gap dielectrics like sapphire ${ }^{3}$. This prevents a dynamic calculation of the pump-pulse-fluence and depth-dependent band-gap shrinkage, thermalization and population difference. As we are mostly interested in the amplification process itself, we will calculate the intraband-thermalization and propagation of the probe pulse assuming a $6 \mathrm{eV}$ band-gap:

To account for the band gap shrinkage in our simple model, we introduce a Heaviside-Theta function $\Theta\left(N^{*}-N\right)$ to the nonlinear term in equation 10.1, which is 1 when a certain conduction-bandelectron density $\left(N^{*}\right)$ and the required band-gap shrinkage is assumed to be reached. When taking this approximation into account, the gain achieved at the front of the material reaches the rear side as seen in figure $10.1 \mathrm{c}$ as the green line. As an input for the simulations, we used a combination of $\sigma_{n}$ and $I$ such that a similar maximum (local) transmission inside the sample was achieved in the 6 and $9 \mathrm{eV}$ band gap cases.

\footnotetext{
${ }^{2}$ Please note that pump and probe fluence were chosen in this simulation to obtain high local amplification.

${ }^{3}$ For silicon, approximations of a carrier-density related band gap renormalization have been made. They are discussed in [92]. However, as silicon is a low band gap semiconductor, we waived to use the approximation for sapphire.
} 


\subsection{Simulations of LADIE and comparison to the experiment}

When the carrier-induced band gap shrinkage, as described above, is taken into account in the simple propagation model, all main experimental observations can be reproduced in a very satisfactory manner. Please note that the local simulated transmission shown is always an integrated transmission of a monochromatic $(402 \mathrm{~nm})$ probe pulse with a FWHM pulse duration of $43 \mathrm{fs}$. The simulated CCD images are simply extracted from the simulated local pump pulse fluence dependence (i.e. the pump dependence was simply mapped onto a spatial Gaussian profile).

Figure $10.2 \mathrm{a}$ shows simulated CCD images of the probe pulse transmission at different time steps: before- and shortly after excitation 4 , as well as after the electronic system has thermalized ( $>100 \mathrm{fs})$. The results are in excellent agreement with the experimental observations shown in figure $9.2 \mathrm{~b}$. We used a pump fluence of $3.1 \mathrm{Jcm}^{-2}$ and a qualitative probe fluence of 0.65 arb. units to obtain the best possible agreement of simulations and experiment. The simulated pump and probe fluence dependencies are described in the following:

In figure $10.2 \mathrm{~b}$, simulated transmission CCD images at three different pump-pulse fluences are shown, whereas figure 10.2 s shows the simulated local pump-fluence dependent transmission for three different probe-pulse fluences. The CCD images as well as the local dependencies capture the main features, from the initial absorption to the amplification for a certain energy range as well as the local fluence dependency that leads to the appearance of a ring-like amplification structure, as it was observed in the experiment (figure 9.4). The initial decrease of the local transmission is slightly less steep compared to the experimental results shown in figure 9.4. We think that the missing three-photon absorption process reducing the transmission at this range of pump-fluence, shown in figure 9.5, is responsible for not capturing this feature. This assumption is supported by comparing the simulation at a low probe pulse fluence (red squares in figure 10.2 k) to an experiment also using a low probe pulse fluence, shown in figure 16.6. The slopes are nearly identical, showing that only free carrier absorption is present.

We also simulated the transmission in dependence on the applied probe-pulse fluence for a pump fluence at which we obtained maximum amplification in the simulations (i.e. $2.65 \mathrm{Jcm}^{-2}$, see figure 10.2 c). In figure 10.3 , we observe a behavior very similar to the one observed in the experiment presented in figures 9.5 and 9.6. For a low probe fluence, the transmission of the probe pulse through the sample is very low. Increasing the probe fluence will at first only slightly increase the transmission, whereas for an ever increasing fluence the transmission drastically rises attributed to the self-reinforcing nature of the stimulated two-photon emission process. However, we are also noting a difference: the unrestricted growth of the local transmission for high probe pulse fluences is not exhibited in the experiment. We think that the major reasons is the negligence of the dynamic carrier-induced band gap shrinkage. Furthermore the purely monochromatic simulation and the missing three-photon absorption process might also lead to a difference between the qualitative behavior of experiment and simulation.

\footnotetext{
${ }^{4}$ Please note that the "before" thermalization simulation was realized by only including the free-carrier absorption in the propagation simulation.
} 

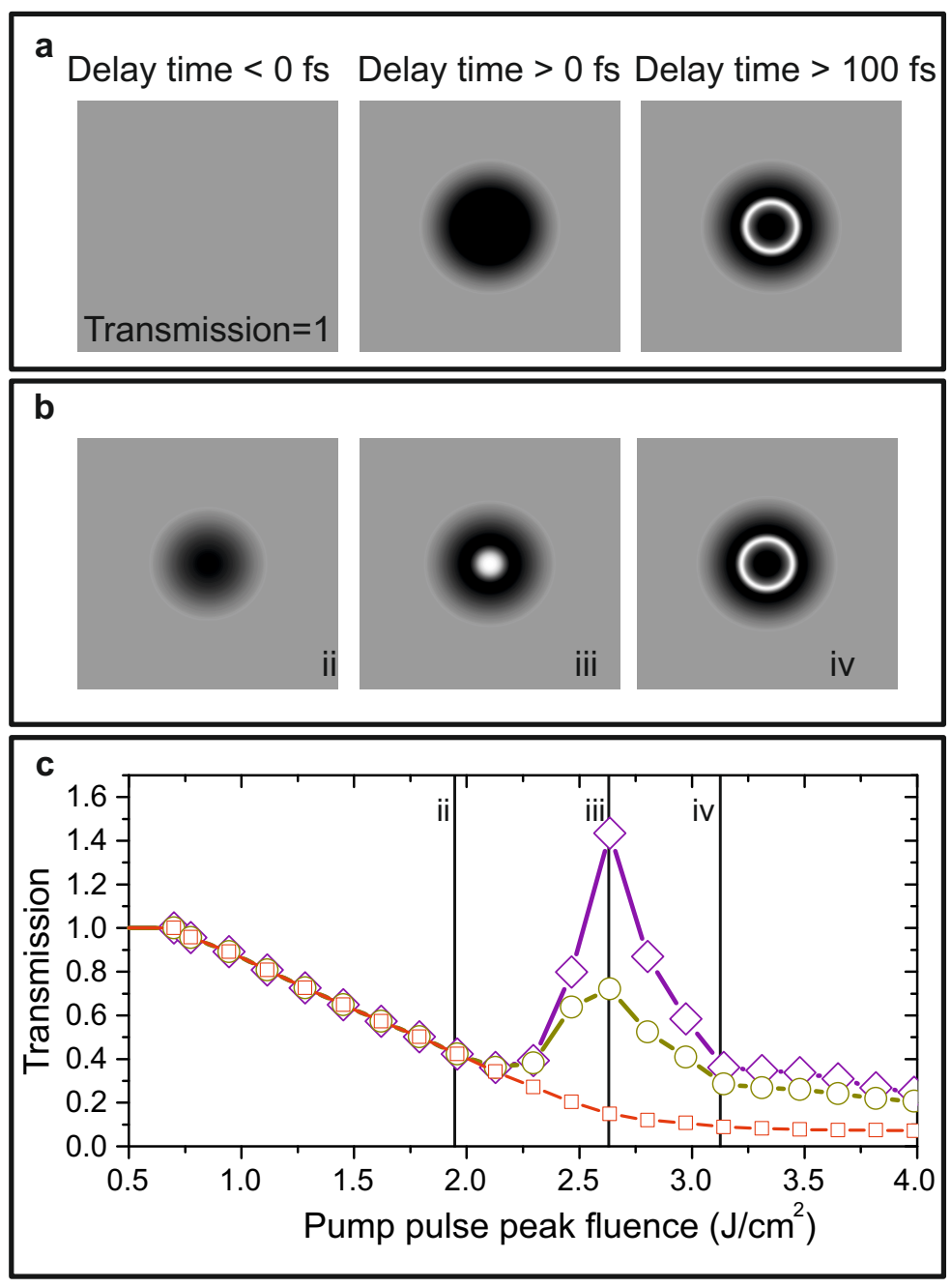

Figure 10.2.: a, Simulated CCD transmission images simulated before, shortly after excitation and after thermalization under amplification conditions (compare to figure 9.2p). b, Simulated transmission CCD images for the indicated pump-pulse fluences (ii, iii, iv) in c. c, Simulation of temporally integrated local probe-pulse transmission for three different initial probe peak intensities showing the dependency on the local pump-pulse fluence. The qualitative probe pulse fluence is indicated by the size of the symbols (red squares $\ll$ yellow circles $\leq$ purple diamonds). See corresponding experimental data in figure 9.4 . 


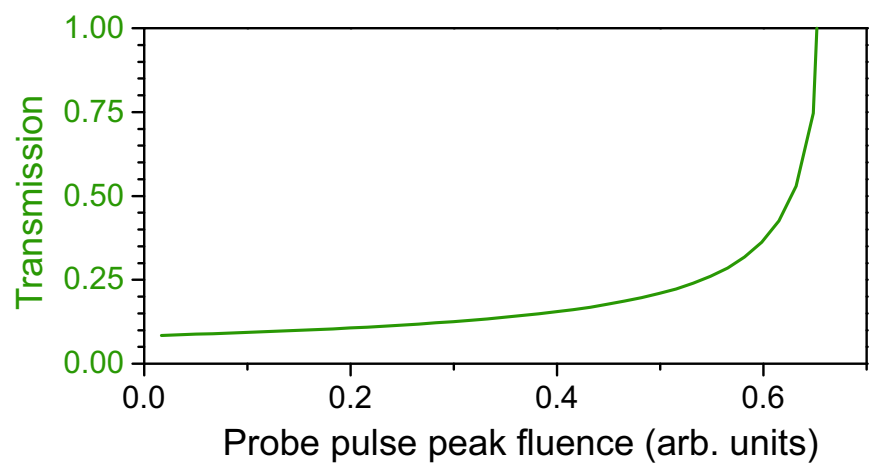

Figure 10.3.: Simulated transmission in a qualitative dependence on the incident probe-pulse peakfluence in the case of amplification (iii in figure $10.1 \mathrm{c}$ ). The transmission is the timeintegrated value for the probe pulse with a FWHM duration of $43 \mathrm{fs}$, calculated $300 \mathrm{fs}$ after excitation and thermalization.

\subsection{Optimizing the amplification by using temporally shaped pump pulses}

So far, we have demonstrated that the amplification process is presumably based on a stimulated two-photon emission process caused by an energetically localized population inversion between the bottom of the conduction- and top of the valence band in dielectric materials ${ }^{5}$. Therefore, the local amplification should be dependent on the "gain" length, i.e. the length over which populationinversion is achieved (see chapter 6). To further confirm that our proposed mechanism is dependent on the excitation depth, we make use of temporally Airy pulses (TAP) described in section 1.2 . In part III, TAP pulses are used to increase the depth of excitation while decreasing the area of excitation [12]. Furthermore in [13] we show that nano-channels with a huge aspect ratio could be produced in fused silica using TAP pulses. Similar results were obtained in sapphire as well [93].

To verify the dependence on excitation depth, we recorded CCD images of the probe pulse for different temporal shapes of the pump pulse, shown in figure $10.4 \mathrm{a}$. We utilized $30 \mathrm{fs}$ bandwidthlimited $\left(0 \mathrm{fs}^{3}\right)$ and temporal Airy pump pulses with parameters of 100, 150 and $250 \mathrm{fs}^{3}$. An increasing TAP parameter leads to a stronger temporal asymmetry, as well as lower peak intensity and longer pulse duration, discussed in chapter 1 . To allow a real comparison, the pictures were taken using adjusted pump-pulse fluences in which maximum local amplification for each pulse shape was reached. This is required as the TAP pulses, which are elongated in time, have a lower peak intensity, meaning that the excitation threshold is reached at higher fluences, shown and discussed in part III. The applied pump fluences for the pulse shapes were all chosen to be below the ablation thresholds.

At first, the CCD images for all pulse shapes look very similar to the ones in figure 9.2 $\mathrm{b}$ : In comparison to the unexcited case, shown in the inset in figure $10.4 \mathrm{p}$, areas of local absorption and local amplification are visible, as well as redistribution of light at large radii. However, for an increasing TAP parameter, two observations can be made in addition: First, the bright areas are located closer to the centre of the probe pulse, which can be explained by a reduced area of excitation caused by the interplay of the nonlinear and linear excitation mechanisms, discussed in part III. Second, and even more importantly, the transmission within the ring strongly increases

\footnotetext{
${ }^{5}$ As in semiconductor laser
} 


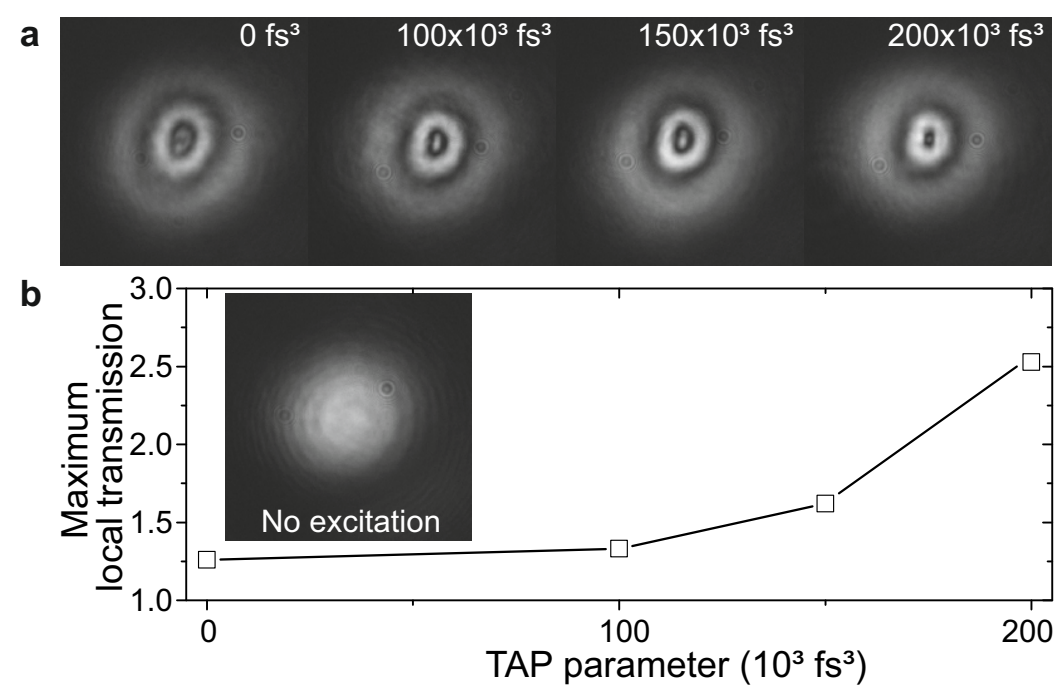

Figure 10.4.: Pulse-shape dependence of the local amplification in excited sapphire: a, Pumpprobe CCD images of the probe pulse for different temporal shapes of the pump pulse. The TAP parameters are indicated in the CCD images. A reference image of the probe pulse for no excitation is given as inset within (b). b, Maximum local transmission as a function of the TAP parameter. The experimental parameters were: Probe pulse peak fluence: $35 \times 10^{-3} \mathrm{Jcm}^{-2}$. Pump pulse fluence for $0 \mathrm{fs}^{3}$ : $2.6 \mathrm{Jcm}^{-2}$; for $100 \times 10^{3} \mathrm{fs}^{3}: 1.46 \times 2.6 \mathrm{Jcm}^{-2}$; for $150 \times 10^{3} \mathrm{fs}^{3}: 1.6 \times 2.6 \mathrm{Jcm}^{-2}$; for $200 \times 10^{3} \mathrm{fs}^{3}: 2 \times 2.6 \mathrm{Jcm}^{-2}$. Pump probe delay time was set to $1.5 \mathrm{ps}$.

with increasing TAP parameter. As this might not be very obvious from the CCD images, figure 10.4 shows the maximum local transmission within the ring as a function of the TAP parameter.

These results indicate that the amplification is strongly dependent on the depth of excitation and correlate well to the system of population inversion discussed in chapter 6 . While these indications are purely derived from our experience and former investigations of laser excitation of dielectrics using temporally shaped laser pulses [12,13], the correlation between excitation (gain-) depth must be verified by simulations in the future. 



\section{Short summary and outlook}

In summary, we performed temporally- and radially resolved (reference-)pump-probe measurements on thin sapphire and fused silica. In these measurements, we were able to observe that a time-delayed $400 \mathrm{~nm}$ probe pulse is coherently amplified inside the dielectrics that were excited by an intense $800 \mathrm{~nm}$ pump pulse.

We found that the amplification is strongly coupled to the presence of conduction band electrons and thus their different lifetime in sapphire and fused silica. Furthermore, a certain strength of excitation, i.e. a certain pump pulse fluence, is necessary for amplification to take place. Amplification was found to be of nonlinear order. If a low probe fluence was used, the expected and well known metallic character of the excited dielectrics was measured, whereas with increasing probe fluence the transmission increased more and more (for a certain pump fluence range). In addition, we found hints of nonlinear absorption and amplification in the spectrum of the probe pulses, depending again on the strength of excitation. For increasing excitation, we found that the amplification extends towards higher wavelengths.

In terms of temporal dynamics, we found that the amplification effects takes around 100-200 fs to reach its maximum while the phase shift, on the other hand, reaches its maximum decrease directly after the interaction with the pump pulse. The amplification lasts over $50 \mathrm{ps}$ in sapphire and only $100 \mathrm{fs}$ in fused silica, due to the respective lifetimes of the conduction band electrons.

When we used temporally shaped laser pulses, i.e. temporal Airy pulses, which are known to increase the depth of excitation inside dielectric materials, we observe a significant increase in the amplification factor.

Thus, the amplification process is not only controllable in space, due to the nonlinearity of the excitation and amplification process and the possible utilization of temporal pulse shapes, it is also controllable in time, given by the natural lifetime of the conduction band electrons and thus the lifetime in which conditions for stimulated emission are given.

At the end, we developed a three-step process of the amplification process that is based on ultra fast band gap shrinkage, intraband-thermalization leading to quasi-Fermi distributed electrons and holes and a subsequent two-photon transition of the probe pulse which can be absorption or stimulated emission depending on the difference of electron densities in the CB and VB at the transition energies, equalling two probe pulse photons. However, at the excitation strength at which the band gap shrinkage is strong enough to allow the two-photon transition, the conditions for population inversion are usually already reached.

We simulated the excitation, intraband-thermalization, and propagation through the excited dielectric including the two-photon process and obtained a very good qualitative and even quantitative agreement with the experimental results, supporting our model. 
While the discovery of an amplification process in dielectrics is in itself a paradigm change and provides a new insight into the light-matter interaction, it also offers a great variety of possible applications:

The most obvious and thrilling one is the possibility of using pure dielectric materials as active materials for UV lasers. In the presented case of sapphire, a $6 \mathrm{eV}$ laser could be realized, by e.g. using perpendicular excitation condition and a line focus geometry. When the focus line matches the length of the dielectric it can be excited homogeneously along the full length and thus be brought into LADIE conditions. This would have the advantage that light would not need to pass regions where the excitation conditions for amplification are not fulfilled and strong absorption would take place. When the carrier then starts to recombine under the spontaneous emission of UV-light, amplification will take place for the ones emitted perpendicular to the excitation direction, leading to stimulated emission in a single pass. At the ends of the sapphire, part of the amplified light will be reflected at the sapphire/air interface and be further amplified traveling back. Depending on the lifetime of the conduction band electrons and the size of the sapphire sample the light could be reflected several times and thus lead to a lasing state, as conditions of resonance are always fulfilled for samples that are larger than $400 \mathrm{~nm}$. The amplified light could then be detected at both edges of the sample.

Another important point is that this study represents the first experimental observation of stimulated two-photon emission/gain in a dielectric material. The process itself was proposed shortly after the invention of the laser [94] stating high promises for possible two-photon amplifiers, due to the inherent nonlinearity of the process itself, leading to unique features such as bi-stability, giant pulse generation, pulse compression and quantum properties such as squeezing [95] or special photon statistics [96]. While it only took four years until singly stimulated two-photon emission was measured [97], more than a decade had to pass until fully stimulated two-photon emission was observed [98]. Those and other experiments [99-101] were performed under very special experimental conditions, involving discrete-level atomic systems, often heavily driven by lasers. Those experiments had different drawbacks: Due to the heavy optical pumping, many competing nonlinear processes appeared, such as self-focusing, stimulated anti-stokes Raman scattering and parametric wave mixing [95]. Furthermore, due to the low density of material present, the obtained gain was very low. Thus, two-photon stimulated amplification was proposed to be much more efficient in semiconductors due to their much higher density [102]. While two-photon absorption was an effect studied in great detail in many experiments, the contrary process was only measured in a few occasions: In complex nanostructured semiconductor materials, based on GaAs, two-photon gain can be obtained in different ways. In the most simple experiment yet, AlGaAs is excited by current based carrier injection and probed by an $800 \mathrm{~nm}$ pulse [103]. Depending on the carrier injection current, the seed pulse either experienced two-photon absorption, two-photon transparency ${ }_{1}^{1}$ or degenerate two-photon stimulated emission. "Degenerate" indicates that two photons of equal wavelength stimulated the two-photon emission, such that at the end four photons are transmitted. Thus, the LADIE effect discovered in this study is thus also presumably based on degenerate two-photon stimulated emission. There also exist nondegenerate two-photon stimulated emission in which two photons of different wavelengths are used to seed and to be amplified, recently observed for the first time in a semiconductor [104]. Thus, the observation of coherent nonlinear amplification an excited dielectrics, as shown in this study, is -to our knowledge- the only straightforward pump-probe experiment applied to a very simple sample system, only consisting of a thin glass slide, allowing the study of this thrilling and mostly hidden process. Therefore it would

\footnotetext{
${ }^{1}$ In this condition, the population of electrons at the transition energy is identical.
} 
be an interesting task to study different aspects of multi-photon stimulated emission by investigating different sample systems providing different band gaps and varying the incident probe/seed wavelength. 



\section{Part III.}

\section{Spatial properties of electronic excitation in water after interaction with temporally shaped femtosecond laser pulses}





\section{Introduction and outline}

$T^{\mathrm{N}}$ our previous study [11], we investigated the pulse-energy and pump-probe delay time depen1 dent dynamics of laser excitation in the central spot of the laser excitation, similarly to what is presented in part II Those studies helped to understand why different temporal pulse shapes lead to different thresholds of laser-induced excitation and optical breakdown in water.

Furthermore, we observed that the measured change in the real part of the refractive index, i.e. the phase shift (see chapter 7), being proportional to the carrier density was higher for temporally shaped (see chapter 1) than for ultrashort, bandwidth limited laser pulses. In our opinion, a first indication that the shaped pulses lead to a higher excitation inside the water jet.

Here, we extend those investigations by focusing on the spatial characteristics of the final ${ }^{1}$ excitation distribution using the same ultrashort and temporal Airy pulses. The experimental setup as well as how the optical properties, phase shift and transmission, are extracted from the measurements are described in section 7.2. In contrast to our previous experiment [11] which was performed in a continuous $1000 \mathrm{~Hz}$ mode, averaging the data at a single delay time and energy setting, the current study is performed in a single-shot mode. Just as in part II for each setting of the pump fluence, one shot is made. Furthermore, in the original work [11], we collected the probe pulse using an optical fiber leading it into the imaging spectrometer. By making use of a pinhole in front of the entrance of the fiber, we selected the central part of the beam profile, thus probing only the changes in the peak intensity part of the pump pulse. In this study, the changes in the optical properties of the excited water sample, i.e. phase shift and transmission, are measured along a line transversely through the beam profile in an imaging spectrometer, the radial dependence is extracted, as described in part III.

To clarify the hints towards different spatial properties of excitation by shaped and unshaped laser pulses, we first measure the pump-fluence dependence of the laser excitation for bandwidth limited (section 13.1) and temporal Airy pulses (section 13.2) at respective pump-probe delay times. This is $300 \mathrm{fs}$ for the BWL laser pulse and $3000 \mathrm{fs}$ for the TAP pulse with a $\phi_{3}$ of $600000 \mathrm{fs}^{3}$ as indicated in figure 12.1 (see chapter 1] and reference [11] for more details). The temporal dynamics following the excitation by the pump pulse are identical to those observed in sapphire, shown in part III. Thus, we will only show and discuss the pump-fluence dependence. Nevertheless prior to the measurements, it was made sure that correct delay times were selected.

Before turning to a direct comparison between the pulse shapes, we compare the fluence dependence of the phase shift in the center $(r=0 \mu \mathrm{m})$ with the one taken from a radial (fluence) line. The observations we made are discussed in the context of propagation effects, which are also discussed for the solid dielectrics in section 16.3 .

In order to determine the spatial extent of the phase shift, we first evaluate the measured radial distribution at fluences where the phase shift in the central part is equal for BWL and TAP laser

${ }^{1}$ All excitation processes, multi-photon and avalanche excitation, are assumed to be finished. 


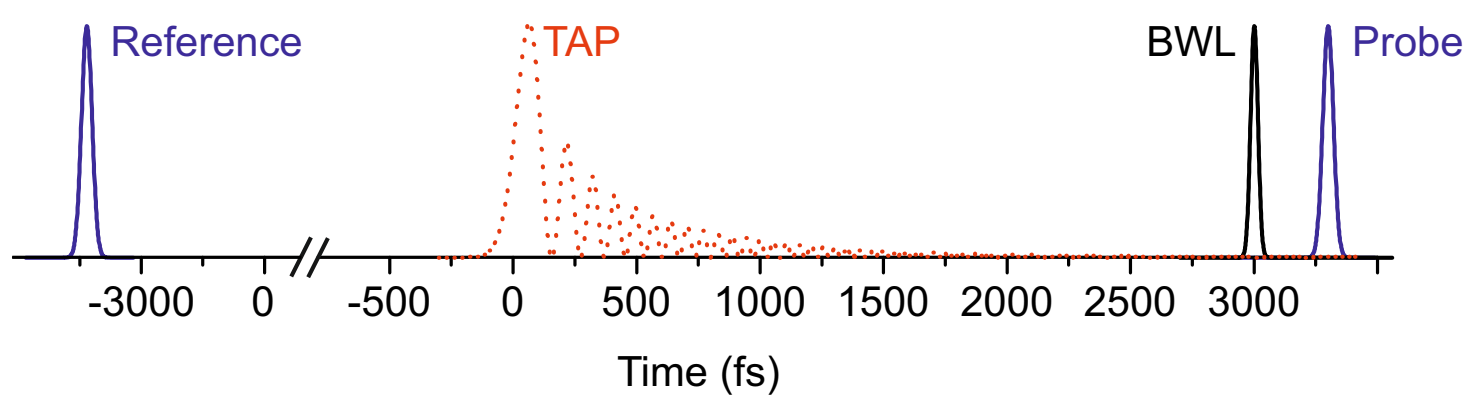

Figure 12.1.: Sketch temporal timing of the laser pulses in the spectral-interference measurements on water as described in the text.

pulses. We then used these excitation conditions as an input for a simple rate equation simulations to compare simulated and measured phase-shifts (14.1). We find a much lower electron density at the surface for TAP pulses compared to the BWL under the given experimental conditions. Differences in the distribution of the surface electron density and the accumulated phase shift are revealed by discussing the depth-dependent excitation at these excitation conditions.

To relate the measurements and simulations to the aim of laser material processing, we simulate the depth and radially-dependent electron density for the situation where the TAP has the same excitation at the surface as the BWL laser pulse (14.1). Finally, the measured and simulated radiallydependent phase shift accumulated over the sample thickness and simulated radially-dependent surface-electron densities are shown in the case of equal excitation at the surface. 


\section{Experimental results}

When the water jet is hit by our ultrashort and highly intense pump-pulse ( $800 \mathrm{~nm}, 35 \mathrm{fs}$ FWHM), conduction band electrons are created via strong-field excitation (see chapter 3 ) and the optical properties change instantaneously, as described in chapter 4 . When those, i.e. phase shift and transmission, are probed with our ultrashort $400 \mathrm{~nm}$ probe pulse as a function of the pump fluence we made different observations:

\subsection{Bandwidth limited laser pulses}

Figures 13.1 and 13.2 show the pump fluence dependence of the central part of the phase shift (black circles) for bandwidth limited laser pulses. The threshold fluence for excitation is reached around $0.2 \mathrm{Jcm}^{-} 2$, where the phase shift starts to decrease to its minimum value of around $-3 \mathrm{rad}$ (figure 13.1(a)). Reaching the maximum change of the phase shift is usually related to an excitation level for optical breakdown [11,39], or the ablation threshold of the material (see III). The observed power law scaling, extracted only from the central part of the pump pulse, in a regime of the phase shift between 0.1 and $1 \mathrm{rad}$ is $6.2 \pm 0.2$, indicated by the black dotted lines in figure 13.2. This directly correlates to the estimated band gap of $8.3 \mathrm{eV}$ (see chapter 2). However, close to the detection limit of the experiment, between 0 and $0.01 \mathrm{rad}$, we measured an excitation of the sample with a lower order process than the main contribution (figure 13.2). This is in accordance with lower excitation mechanisms in water [105, 106]. As we only have a limited amount of data points in this regime, we are not able to provide a power law scaling fit that could reveal a possible band gap energy of this excitation process. Remarkable is that the excitation by this lower order process seems to saturate until the main excitation process sets in at around $0.3 \mathrm{Jcm}^{-2}$, which could indicate that this excitation process is the one provided by the solvated electrons, which are said to only be able to provide a limited number of electrons, as introduced in chapter 2. Thus, we were able to observe a pump pulse fluence dependent transition from two distinct photo-excitation processes. The phase shift at which the first excitation saturates is around $0.05 \mathrm{rad}$, which correlates to an electron density of just $1.4 \times 10^{18} \mathrm{~cm}^{-3}$ assuming a constant electron density (equation 7.3 with a constant scattering time of $1.6 \mathrm{fs}$ ) along the propagation direction of the laser pulse, which is valid in this regime [11]. It is noteworthy that the electron density inside the sample is not high enough to provide a detectable change in transmission.

With increasing fluence, the phase shift starts to increase again, visible as a bend of the slope in figure 13.1 $\mathrm{k}$. The reason for this behavior is still unclear and was so far, not observed, to that extent, in any spectral- or spatial interference experiments on solid dielectrics [57,66, 70]. Even in our measurements on sapphire (part III), the increase in phase shift is significantly lower. It might be related to the influence of the decreasing valence band electron contribution to the dielectric function, described via the Clausius-Mossotti relation (see section 4.2), due to an increasing reflectivity at the surface drastically reducing the fluence of the pump pulse entering the sample or 


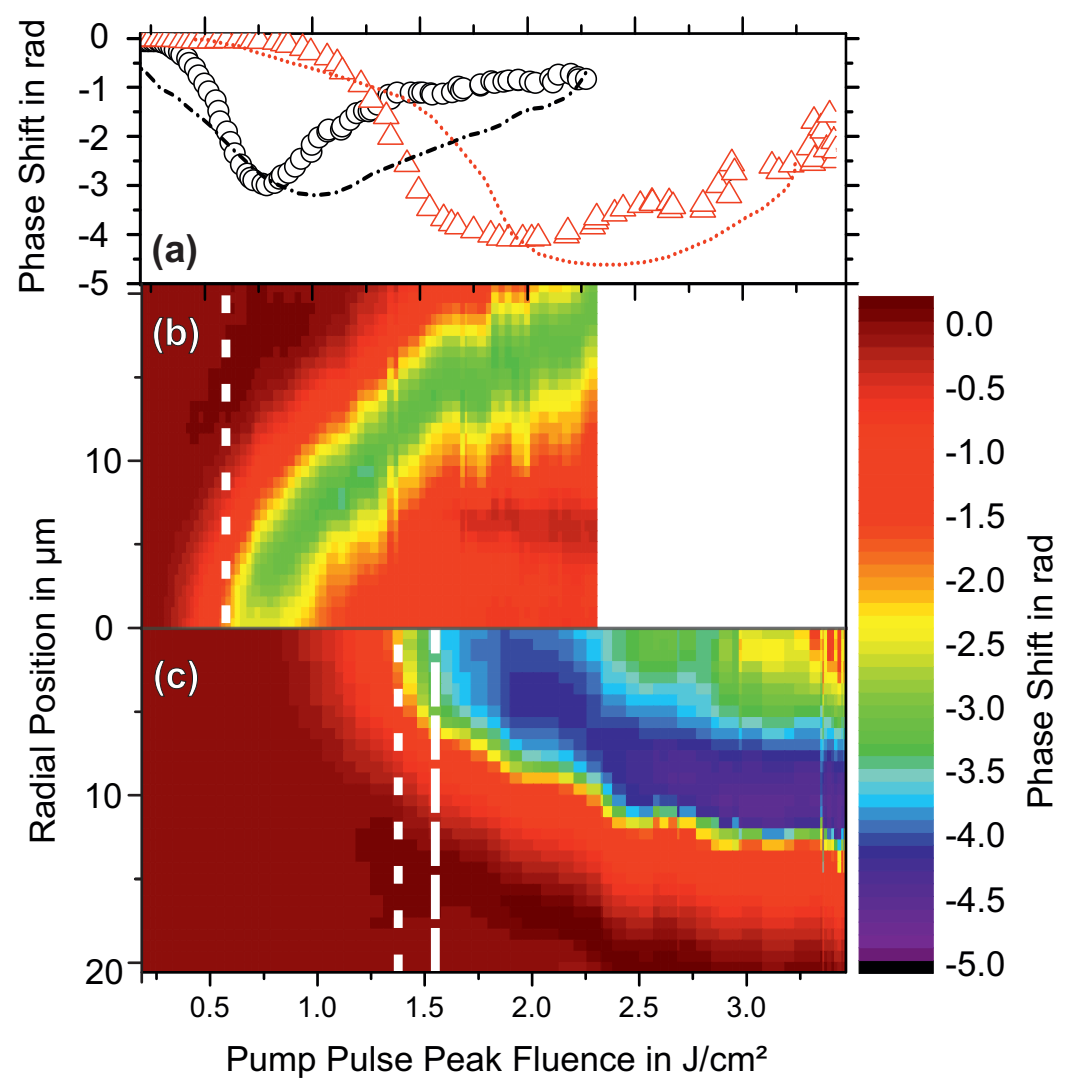

Figure 13.1.: a, Pump pulse fluence dependence of the phase shift in the central part of the pump pulses (BWL black circles, TAP red triangles). The red dotted line represents the fluence dependence of the phase shift for the TAP pulse extracted from the radial distribution taken at a peak fluence of $3.25 \mathrm{Jcm}^{-2}$, the black dash-dotted line the corresponding distribution for the BWL laser pulse extracted at a peak fluence of $2.25 \mathrm{Jcm}^{-2}$. b, Radially-resolved phase shift measurements as a function of pumppulse-fluence for the BWL laser pulse. c, same as (b), using the TAP pulses. Dashed vertical lines in (b) and (c) indicate positions where radially-dependent phase shift data for figure 13.2 were extracted. The measurements in (a) are averaged values of the phase shift over $\pm 3.25 \mu \mathrm{m}$ in the center of the pump pulse (b) and (c). 


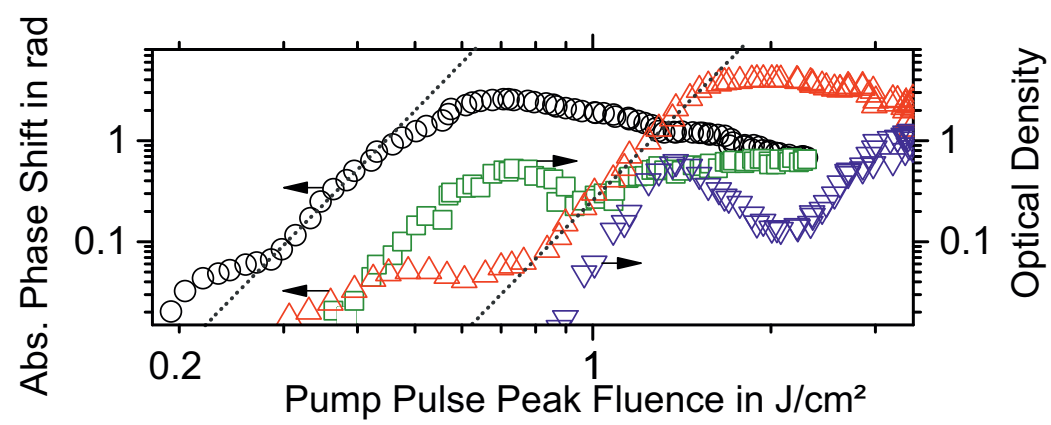

Figure 13.2.: Double-logarithmic representation of the measured absolute phase shift and optical density for BWL (circles and squares) and TAP pulses (up and down pointed triangles) extracted from the central part in figure 3. The black dotted lines indicate a power law scaling of 6 . Please note that the measured phase shift is negative.

due to propagation effects of the pump or probe pulse 4.5). At this point, a more complex and detailed theoretical approach is needed to understand these processes in the highly excited material, as our simple single rate equation model is only valid in the excitation regime below the optical breakdown as will be shown within the next sections.

With respect to the spatial extent of the laser excitation, we observe in figure $13.1 \mathrm{p}$, that with an increasing pump fluence, the change in phase shift moves towards greater radii following the spatial intensity profile (Gaussian) of the pump pulse.

In addition to the phase shift, the optical density (OD), which is the logarithm of the transmission (see 4), was measured and the results for the central part is shown in figure 13.2 (green squares). As observed previously [11], the onset of the OD is shifted towards higher pump fluence and follows then the same power law scaling as the phase shift. With increasing pump pulse fluence, the optical density undergoes a minimum, which was also reported in our previous investigations. Until now, this behavior was not observed elsewhere and its origin is linked to the recently discovered "laser amplification in excited dielectrics" effect, discussed in part III. At the time when these measurements were performed, we were not aware of this phenomena and neither knew the dependency of the enhanced transmission on the incident probe pulse intensity.

From the optical density and phase shift, the scattering time was extracted as presented in [11] to be $1.6 \mathrm{fs}$ from the region in which both quantities are following the power law $(0.1$ to $0.9 \mathrm{rad}$ of the phase shift). We assume, that the contribution to the scattering time by the carrier density dependent term of eq. 5.8, to be neglectable (see section 5.1). The result is consistent with our previously reported value of $1.6 \mathrm{fs}$ [11]. 


\subsection{Temporal Airy pulses}

Pump-pulse-fluence dependent measurements are performed using positive TAP pulses, as described in chapter 1 . In figure $13.1 \mathrm{c}$ the radially-resolved and pump-pulse-fluence dependent phase shift is shown.

The onset of the changing phase shift is shifted by a factor of around 2.2 towards higher fluences compared to the BWL laser pulse (figure $13.1 \mathrm{p}$ ). This is consistent with our previous investigation [11] and the reason is found in the drastically decreased peak intensity of the shaped pulse (by a factor of around 6), as seen in chapter 1 .

As in the case of the BWL pulse, in the center of the beam, shown in figure 13.1 (red triangles), the phase shift starts to decrease until a maximum change of around $-4 \mathrm{rad}$, being significantly higher compared to the results obtained when using the BWL pulse $(-3 \mathrm{rad})$. With increasing fluence, the phase shift starts to increase again. However, the increase is smaller as compared to the bandwidth-limited pulse. In the phase shift range of 0.1 to $1 \mathrm{rad}$, the slope follows the same power law of $6.2 \pm 0.2$ as the BWL laser pulse, indicated by the black dotted line in figure 13.2 . This might not be very intuitive, since the TAP pulse has a much higher pulse duration, however, close to the excitation threshold multi-photon excitation is the driving process, leading to an equal power law.

Similar to the case of the BWL, we observe an excitation process of a lower order. The onset of this process is only shifted by 1.5 times towards higher fluences and shows a saturation that persists for over $0.5 \mathrm{Jcm}^{-2}$ before the main excitation process, following the power law of $6.2 \pm 0.2$, starts.

The measurement of the optical density for the TAP pulse shows the same behavior as the corresponding one for the BWL. In comparison, the optical density shows a much more pronounced minimum, which occurs over a larger fluence regime for the shaped pulse. The reason for the different strength of the reduced absorption, i.e. increased transmission, is again related to the LADIE effect. By using the shaped pulse the conditions in which amplification of a probe pulse is obtained area fulfilled for a larger depth compared to BWL laser pulses, as discussed in part II.

\subsection{Fluence dependence of the phase shift: Center vs Radial dependency}

Before we directly compare the spatial distributions of phase shift by the two pulse shapes, we compare the fluence dependence of the phase shift measured in the center with the phase shift obtained from the radial measurement, just as we did for the measurements on the solid samples (see 16.3).

As in a cut along the radial distribution of the phase shift all pump pulse fluences are contained (as a function of radius) due to the Gaussian pulse shape, it is valid to compare it directly to a measurement taken from the center (see figure 13.2 a). Thus, in a fist approximation, the two measurements should give identical results when shown on the same local fluence scale.

In figure 13.1 a the fluence dependent phase shift for the center line and for the radial cut at $2.25 \mathrm{Jcm}^{-2}$ for the BWL (black dotted line) and $3.25 \mathrm{Jcm}^{-2}$ for the TAP pulse (red dash-dotted 
line) are shown. The qualitative shape of the two data sets is similar, however, the radial distribution is less steep. This discrepancy may be attributed to nonlinear propagation effects that occur at higher fluences, as plasma-defocusing of the probe pulse and Kerr-induced self focusing of the pump pulse can change the beam waist of the laser pulse in that regime (see section 4.5).

Even though in the present experiment the power for self-focusing is exceeded by more than one order of magnitude, the self-focusing length i.e. the length over which the beam collapses, is larger than the sample thickness [53]: We consider the highest fluence used for the BWL laser pulse which is around $2.5 \mathrm{Jcm}^{-2}$. Here, the applied power of $142 \mathrm{MW}$ exceeds the critical power for self-focusing of water at $785 \mathrm{~nm}$, with a nonlinear refractive index of $1.9 \times 10^{-16} \mathrm{~cm}^{2} \mathrm{~W}^{-1}$ [107], by nearly a factor of 40 . Nevertheless the resulting self-focusing length [53] of $723 \mu \mathrm{m}$ is more than seven times larger than the sample thickness, being only around $100 \mu \mathrm{m}$. For the TAP laser pulses the applied power is even lower due to the increased pulse duration [13].

Regarding the changes of the phase shift, we also observe that the maximum change is higher for the radial cut with 10 percent more for BWL pulses and 20 percent more for TAP pulses. This may indicate that linear- and nonlinear propagation of the pump beam can lead to higher electron density inside the material. Besides the pump, also the probe pulse can be affected by plasma defocusing of a highly excited sample, causing a longer propagation through the sample, possibly accumulating a higher phase shift. This comparison shows the importance and the need for spatially resolved measurements of laser excitation of dielectric materials as the general behavior cannot simply be extracted from a single scan at high fluences. To fully resolve this issue a propagation calculation based on Maxwell's equations [108] is required in order to understand the experimental results. At low and moderate fluences, the radially dependence shows the same behavior as a central line scan. Simulation and experiment agree in this regime very well (see figure $14.1 \mathrm{a}$ and $\mathrm{b}$ ) as will be discussed in the next section. 



\section{Comparison of measurement to simulations}

For a direct comparison of the radially properties of the laser excitation at a peak fluence of $0.61 \mathrm{Jcm}^{-2}$ for the BWL pulse and $1.34 \mathrm{Jcm}^{-2}$ for the TAP pulse is chosen. Under these excitation conditions, an equal accumulated phase shift of around $-2.3 \mathrm{rad}$ in the center of the pump pulse $(r=0 \mu \mathrm{m})$ is measured. In figure 14.1 a the radial distribution of the phase shift is shown. The white dashed lines in figure $13.1 \mathrm{p}$ and $13.1 \mathrm{c}$ indicate where the radial distribution was extracted. Both distributions reach a value of around $-2.3 \mathrm{rad}$ in the center, and both strongly decrease with increasing radius as expected from the Gaussian beam profile providing the local fluence distribution. Despite all the similarities, the change in phase shift decreases much stronger in the case of the TAP pulse as compared to the BWL laser pulse. It appears that the excitation takes place over a smaller area for TAP pulses. As discussed in part I. the measured phase shift is a value integrated over the whole sample thickness and does not represents the excitation at the surface of the water jet (see section 7.2). Thus we performed single rate equation simulations (see chapter 5) for a better understanding of the spatial excitation properties.

\subsection{Radial dependent phase shift}

As a first step, we simulated the radial dependent phase shift after passing the water jet for both, BWL and positive TAP pulses using identical fluences as in the experiment. In figure 14.1 the resulting simulated and measured phase shifts are shown. Simulated and measured values agree well. To reach an equal phase shift in the center part for the TAP pulse, the required fluence is 2.2 times higher than for the BWL laser pulses, in the simulation and the experiment, respectively. The simulation reproduces the phase shift for all radial positions and thus all fluences below this value. This validates our model up to this maximum fluence, that takes multi-photon and avalanche excitation into account (see chapter 5 ).

As the measured phase shift is only an integrated value over the whole sample thickness, we now want to correlate the measured values with the actual excitation of the sample. Thus, we provide the radial distribution of the electron density at the surface $(L=0 \mu \mathrm{m})$ of the water jet using the same fluences that led to the $-2.3 \mathrm{rad}$ phase shift in the center part as shown in figure 13.2 $\mathrm{a}$. In figure 14.2a, the simulated radially-dependent electron densities for BWL (black solid line) and TAP (red dotted line) pulses at the surface of the water jet are shown:

Surprisingly, the spatial differences between the two pulse shapes are much higher than in the phase shift measurement: the maximum conduction band electron density obtained by the TAP pulse is about a factor of 4 lower than the value of the bandwidth-limited laser pulse. In addition, the excitation at the surface is distributed over a smaller radius. 


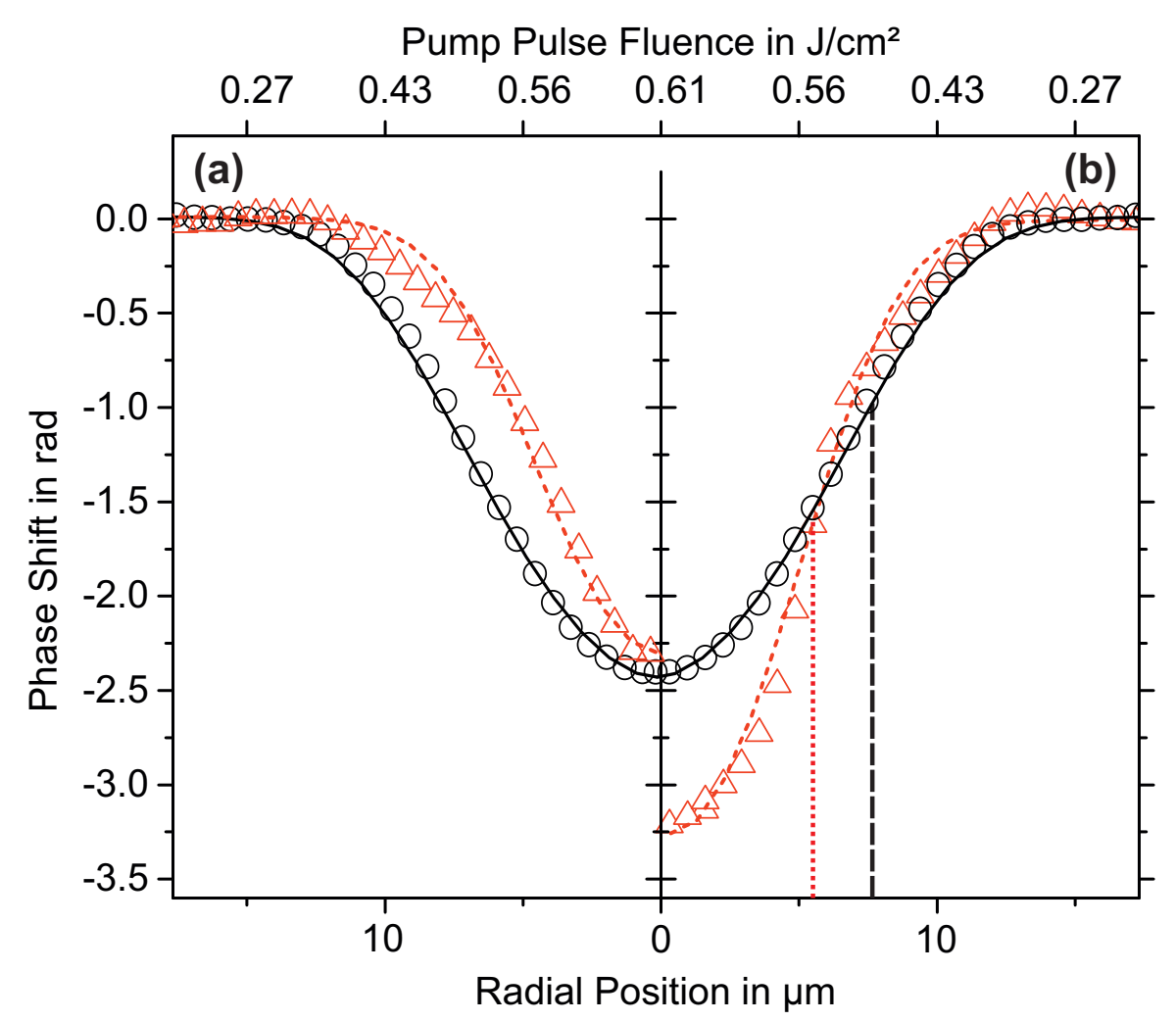

Figure 14.1.: Measured (symbols) and simulated (lines) radially-dependent phase shift for BWL(black) and TAP(red) laser pulses: a, Equal measured phase shift at $r=0 \mu \mathrm{m}$ for both pulse shapes. For the TAP pulse, the fluence is increased by a factor of 2.2. The data was extracted from the lines indicated in figure $13.1 \mathrm{~b}$ for the BWL and figure 13.1c for the TAP (short dashed lines). b, Equal simulated electron density at $r=0 \mu \mathrm{m}$ for both pulse shapes. The fluence for the TAP laser pulse is increased by a factor of 2.5 and the data was extracted from the lines indicated in figure $13.1 \mathrm{~b}$ and 13.1 (long dashed line for the TAP pulse). Black dashed and red dotted vertical lines indicate the radius at which the phase shift decreased to half of the minimum value. 
The discrepancy between the measured phase shift and simulated excitation at the surface is clarified by the depth-dependent conduction-band electron density, shown as the red dashed line for the TAP and the black solid line for the BWL laser pulses in figure $14.2 \mathrm{p}$ :

The electron density decreases for both pulse shapes with increasing depth, caused by the free carrier absorption (chapter 4). However, for the TAP pulse, the decrease is much lower, such that from around $20 \mu \mathrm{m}$, the electron density is higher for the TAP pulse compared to the BWL. Therefore the probe pulse, integrates the strength of excitation over all depths and thus, the accumulated phase shift will be higher for the rest of the sample, giving identical values for TAP and BWL after the pulse passed the full length of the water jet.

For (surface-)material processing experiments, as for example ablation or sustainable refractive index changes it is usually necessary that the electron density at the surface has to reach a certain value, as it is correlated to a certain energy input into the material. This is not valid for materials which exhibit the formation of ultrashort transient defect states (e.g. self-trapped excitons), which drastically changes the interaction of longer laser pulses (like the TAP pulses) with the material. This issue is discussed in detail in our recent work on fused silica in [13].

Therefore we will have a look at the electron density in the central part at $r=0 \mu \mathrm{m}$ of the laser pulses as a function of depth:

As an input for the simulations, we use fluences where an equal conduction-band-electron density is obtained at the surface for both pulse shapes. To that end, we increased the fluence of the TAP pulse by a factor of 2.5 in comparison to the BWL, as shown in figure $14.1 \mathrm{~b}$. This factor was also observed in the processing of fused silica [13] and sapphire [93], where the threshold of ablation was shifted around $2.5 \times$ towards higher pulse energies for the same TAP laser pulse. Thus, a peak fluence of $0.61 \mathrm{Jcm}^{-2}$ for the BWL pulse and $1.52 \mathrm{Jcm}^{-2}$ for the TAP pulse were used.

In figure $14.2 \mathrm{~b}$ the electron density in dependence on the depth is shown for both pulse shapes. Starting from an equal electron density at the surface, the value drastically decreases for both pulse shapes, but for the TAP pulse (red dashed line), the decreases is much less. This leads to a higher excitation inside the material and therefore a much higher phase shift accumulated by the probe pulse.

The reason for the higher excitation inside the material by the TAP pulse can simply be related to the longer pulse duration and therefore the creation of free electrons mainly driven by avalanche excitation (see chapter 3). The strong pulse at the front of the TAP pulse train (chapter 1) creates a relatively low free electron density via multi-photon excitation, whereas the rest of the pulse-train increases slowly the electron density to a much higher value [11, 13, 109]. This also leads to a slower increase of the absorption coefficient, which is proportional to the electron density (see chapter 4), reaching its highest value when the lowest intensity of the pump pulse is present.

Finally, we now compare the radial phase shift distribution in the discussed case of an equal excitation at the surface. In figure $14.1 \mathrm{~b}$ the radially dependent simulated and measured phase shift- for $0.61 \mathrm{Jcm}^{-2}$ (BWL) and $1.52 \mathrm{Jcm}^{-2}$ (TAP) pump-pulse peak fluence is shown. Please note that the change in phase shift at $r=0 \mu \mathrm{m}$ is around 1.4 times higher for the TAP ( $-3.2 \mathrm{rad}$ ) compared to the BWL $(-2.3 \mathrm{rad})$. This factor is similar to the regime where the maximum phase shift change is reached in the center part, as seen in figure 13.1 a. At this state, the BWL pulse has a minimum at $-3 \mathrm{rad}$ and the TAP pulse at $-4 \mathrm{rad}$. This indicates that the spatial properties observed under low and medium excitation conditions are also be valid in the high excitation regime. 


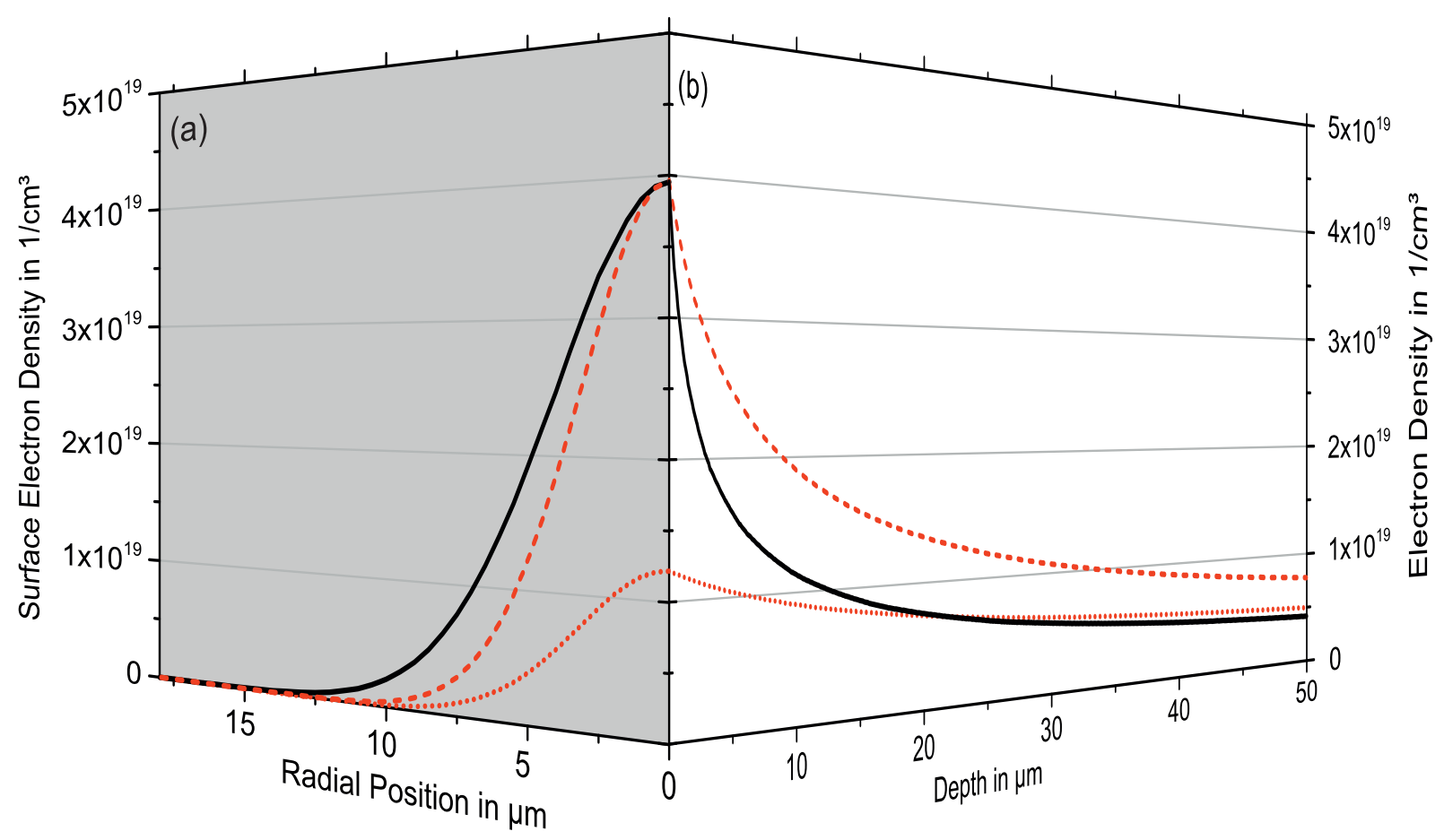

Figure 14.2.: Simulated radially- and depth-dependent phase shift for BWL (black) and TAP (red) laser pulses: (a) Radially-dependent phase shift for: Equal phase shift at $r=0 \mu \mathrm{m}$ (red dotted line), with the same fluence as in figure 14.1 a. Equal electron density at $r=0 \mu \mathrm{m}$ (red dashed line), with the same fluence as in figure 14.1p. (b) Depthdependent conduction-band-electron density at the center of the laser pulse for the same cases as in (a). 
The radial position of the phase shift, as it decreased to half of the maximum value, is smaller for the TAP $(r=5.5 \mu \mathrm{m})$ compared to the BWL $(r=7.6 \mu \mathrm{m})$ pulse, indicated by the dashed (BWL) and short-dashed lines (TAP) in figure 14.1p. Thus, in terms of area, the shaped pulse will excite about half the area compared to the BWL laser pulse. The same relation between BWL and TAP laser pulses is found in the radial distribution of the conduction-band-electron density at the surface at the material, shown in figure $14.2 \mathrm{a}$. The results obtained in this study correlate perfectly to post mortem analysis results of material processing on fused silica [13, 109] and sapphire [110], where the identical temporal pulse shapes were used. In both material systems, ablation structures created from bandwidth-limited laser pulses were relatively large and shallow, whereas the structures obtained by TAP shaped laser pulses were spatially more confined and much deeper. Although the very simple model used here does not quantitatively predict the very large differences in the observed radii of the generated structures in fused silica and sapphire, the observed trends in this investigation correlate well with the materials-processing results. Please note further, that the focusing conditions in this experiment are different to those in the mentioned studies on solids. The beam diameter in the presented study is tens of $\mu \mathrm{m}$, whereas it is only a few in the processing experiment. Thus the Rayleigh ranges drastically differ. 



\section{Short summary}

In summary, radially-resolved, fluence-dependent spectral interference measurements on laser excited water were discussed, using ultrashort BWL and positive temporal airy pulses.

We demonstrated that the measured excitation by TAP pulses is confined to a drastically reduced area compared to BWL laser pulses by almost a factor of two.

By making use of a simple rate equation model, taking into account multi-photon and avalanche excitation, absorption and reflection of the pump pulse, we are able to reproduce the results in an excitation regime below the optical breakdown.

It was further elucidated by the simulations that TAP pulses lead to a higher electron density excitation deeper inside the material as compared to BWL laser pulses. We conclude that TAP shaped laser pulses can control the area and depth of laser excitation.

Overall this investigation confirms that temporally asymmetric shaped laser pulses are a powerful tool for high precision laser material processing as was also recently demonstrated in subwavelength diameter, high aspect ablation structures in fused silica [13] and in the non-destructive optoporation of cells [30]. 
Part IV.

Summary 


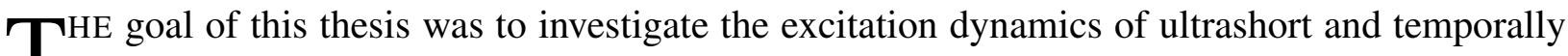
1 shaped femtosecond laser pulses in three drastically different dielectric materials: crystalline sapphire, amorphous fused silica and liquid water.

In order to reach that goal, two complementary experiments where set up and deployed: a spectralinterference and a pump-probe microscope, both using ultrashort $800 \mathrm{~nm}$ pump- and $400 \mathrm{~nm}$ probe pulses. In a purely pump-probe based experiment, measuring and evaluating the transmission of the probe pulse through an excited sample is simple and intuitive. However, this technique is limited to that one physical quantity, that represents the change in the imaginary part of the refractive index of the material. The spectral-interference technique on the other hand is more versatile but also more complex. It is based on a reference-pump-probe scheme in which the probe pulse is spectrally interfered with its replica (reference pulse), that probed the unexcited state of the sample. From this data, even small changes in the real- and imaginary part of the refractive index can be extracted with high accuracy and robustness, allowing to detect a larger variety of electronic dynamics of dielectric materials compared to the pump-probe scheme. Such dynamics are for example the optical Kerr effect or the creation of excitonic states. The most important difference regarding the detection of transmission between the two used experimental techniques is that the spectral-interference is sensitive to coherent emission of light only.

When these techniques were used to study the excitation in thin crystalline sapphire samples, something truly unexpected was observed: The $400 \mathrm{~nm}$ femtosecond probe pulse was under certain conditions amplified locally and even globally, meaning more pulse energy was transmitted through the excited sapphire sample compared to what was incident. This effect has never been observed before, as typically laser-excited dielectric materials absorb light due to the presence of conduction band electrons, representing a transient semi-metallic state. Thus, a thorough systematic study of temporal and energetic dependencies of this effect was performed. It was found that the amplification appears on a time scale of one hundred femtoseconds after excitation, which corresponds to the time of intraband-carrier-thermalization and is only present when a certain level of excitation, i.e. a minimum number of conduction band electrons was created by a sufficiently intense pump pulse. This was further confirmed by probing the excitation dynamics in amorphous fused silica. In this material, the lifetime of electrons is strongly limited due to their trapping into excitonic states on a timescale of a few hundred femtoseconds. As soon as the electrons are trapped into excitonic states, the amplification effect vanishes. But, not only the correct level of excitation is important, as it was found that the amplification process is of a nonlinear order. That means, that a sufficiently high probe intensity must be applied in order for amplification to take place. A detection of amplification in both, pump-probe and spectral-interference measurements proves its coherent nature, making it a "laser-like" process. A three-step model was developed that explains all experimental observations. By simulating each step of the model, an overwhelming qualitative and even semi-quantitative agreement between simulation and experiment was obtained, showing that the amplification process is based on stimulated two-photon emission. A rarely observed effect at all, that has never been seen before in a solid dielectric material.

The discovery of coherent amplification of femtosecond laser pulses in an ordinary piece of glass holds very high promises for future investigations and applications. It could for example be used to realize very simple deep UV laser sources, for highly nonlinear microscopy or even to study the effect of nonlinear stimulated emission in a way, much simpler than it has been used to in the past.

As a final proof of the laser-like amplification scheme, temporally asymmetric shaped laser pulses 
were used, that are known to elongate the excitation inside dielectric materials. An increase in excitation depth should directly transfer to a length over which the material is driven into amplification conditions, i.e. an increase in gain length. By doing so, the amplification effect was strongly increased by several factors, showing that the fundamental processes involved were correctly identified and interpreted.

In the third part of this thesis, the focus was set on these special spatial properties of laser excitation that go hand in hand with the utilization of temporally asymmetric shaped pulses. Here, water as a sample system was used in a spectral-interference measurement accompanied by simple rate equation simulations. It was found that by utilizing the shaped pulse, the area of excitation is reduced by more than a factor of two, whereas the depth of excitation is strongly increased. These results are in perfect agreement with many post-mortem investigations on solid dielectrics that utilized the identical pulse shape and thus show, that the initial distribution of excitation is imprinted to a certain extend onto the final ablation structure. Bandwidth limited femtosecond laser pulses quickly excite a large carrier density, leading to high absorption and reflectivity close to the surface of the material. Temporally asymmetric shaped laser pulses on the other hand, make use of a seed and heat mechanism allowing it to be less affected by absorption close to the surface resulting in a larger depth of excitation and eventually also of ablation. This temporal pulse shaping technique for deep excitation and ablation is solely based on the interplay of excitation mechanisms with the special temporal structure of the laser pulse, being in strong contrast to spatial beam shaping techniques that simply utilize non-diffracting Bessel beams or to techniques that exploit filamentation.

The high potential of temporal pulse shaping techniques for nanostructuring of solid dielectrics was recently demonstrated by the creation of high aspect ratio channels in fused silica. However, it holds also very high promises for non-technical applications such as optoporation of cells or direct nanosurgery which is part of ongoing investigations. 


\section{Part V.}

Appendix 


\section{Additional data to part II}

This part contains additional data that is related to the results discussed in part [II

\subsection{Sapphire}

\subsubsection{CCD Images of the delay scan}

In part $\Pi$ we discuss the pump-probe delay time dependent dynamics, i.e. local and global transmission (see figure 9.2). We also show four CCD images, that were taken at selected delay times. Here, we provide all CCD images taken at each delay time step. The images are split up in two parts and shown in figures 16.1 and 16.2. The CCD images are overlayed with a sketch indicating the temporal separation between pump- (red) and probe (blue) pulse.

\subsubsection{Delay scan using a low pump pulse fluence}

In figure 16.3, a radially-resolved phase-shift measurement as a function of the pump-probe delay time is shown. In this measurement, a very low pump fluence of only $0.5 \mathrm{Jcm}^{-2}$ was used. For times before $-100 \mathrm{fs}$ and after $100 \mathrm{fs}$, a phase shift of $0 \mathrm{rad}$ is observed for all radial positions. Only at times, where an overlap of pump and probe pulses is given, a positive phase shift is observed. This is, as discussed in part III, caused by the optical Kerr effect (see section 4.4), introducing a pump intensity dependent nonlinear refractive index. It can be seen that the phase shift follows a Gaussian distribution in time, as it is caused by a convolution of the temporal shapes of probe and pump pulse and such a measurement is comparable to a phase gating FROG [11]. From such a measurement the pulse duration of one pulse can be obtained, if the duration of the other one is known [11,111].

This relation is given by the FWHM pulse duration of the "Kerr-peak" [79]:

$$
\Delta t_{\text {Kerr }}=\Delta t_{\text {Probe }}^{2}+\frac{1}{2} \Delta t_{\text {Pump }}^{2}
$$

$\Delta t_{\text {Probe }}$ is the FWHM pulse duration of the probe pulse, whereas $\Delta t_{\text {Pump }}$ is the corresponding one of the pump pulse.

The pump pulse duration is usually measured in a GRENOUILLE2 device, which is based on FROG. Then, in the interaction area of the experiment we use a nonlinear photodiode and the pulse shaper to compress the pulse to its shortest duration that was measured with the GRENOUILLE.

\footnotetext{
${ }^{1}$ FROG: Frequency resolved optical gating [111].

${ }^{2}$ GRENOUILLE: Grating-eliminated no-nonsense observation of ultra fast incident laser light e-fields.
} 

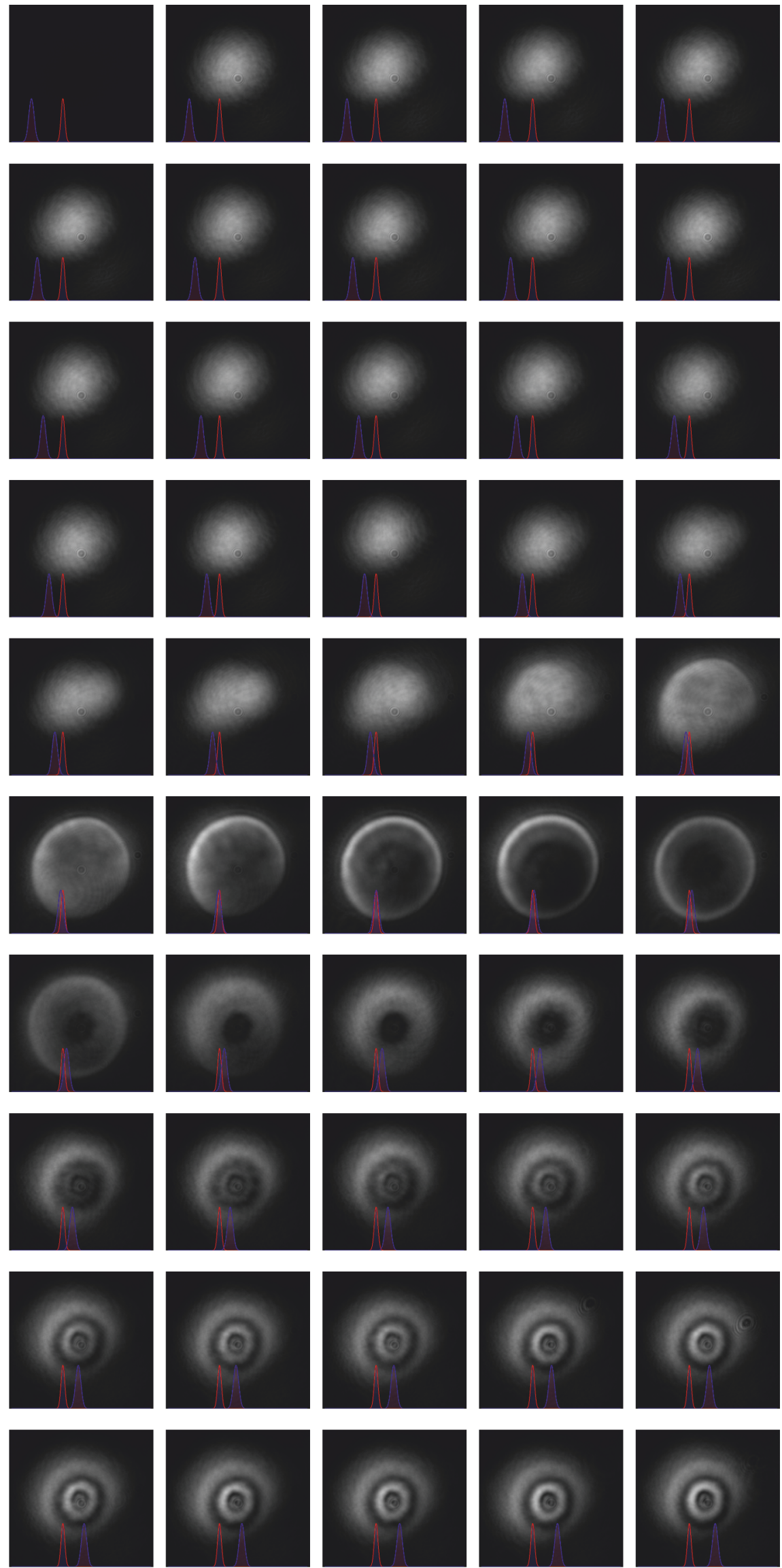

Figure 16.1.: CCD images of the delay scan shown in figure 9.2 . 

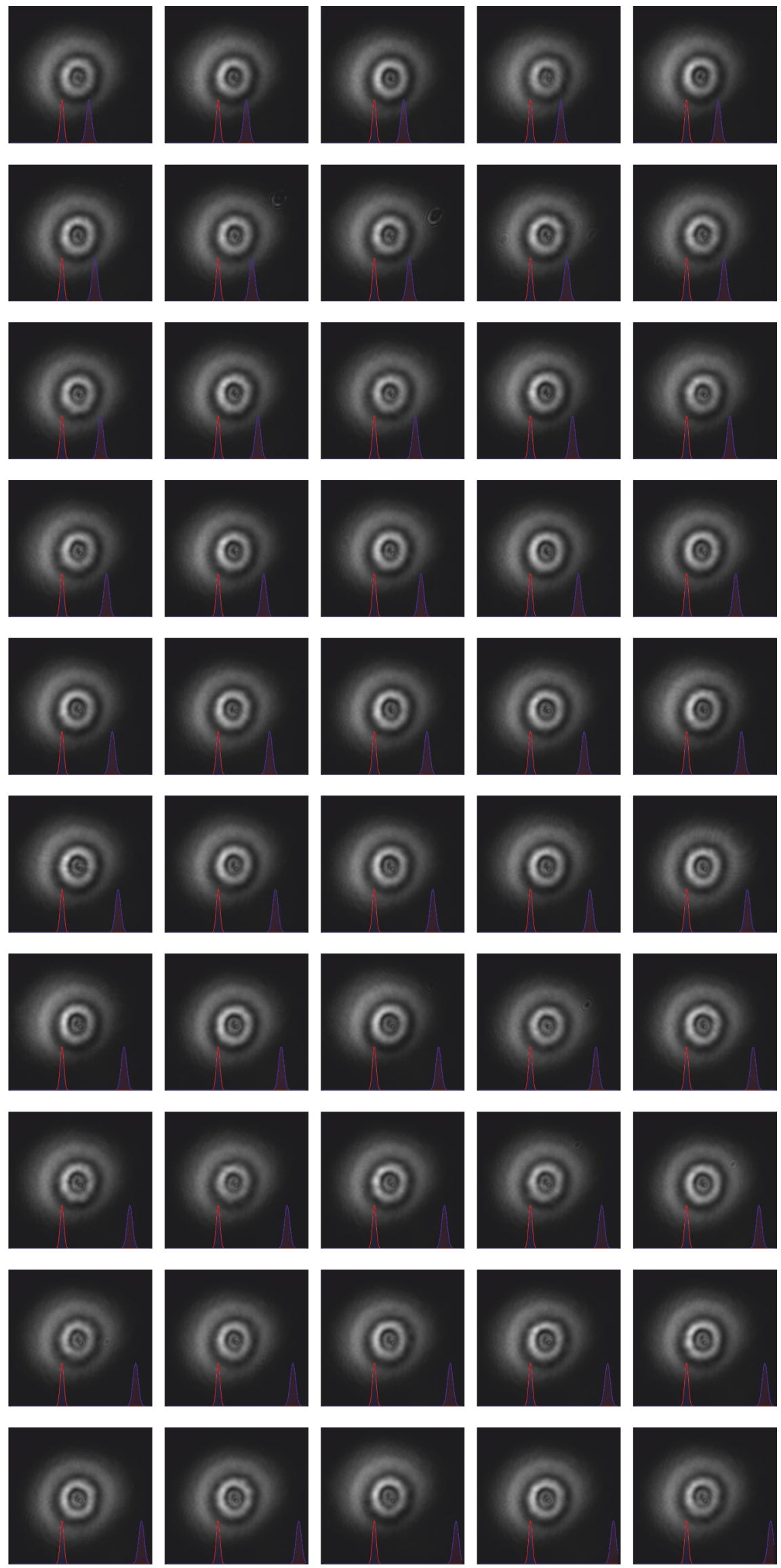

Figure 16.2.: CCD images of the delay scan shown in figure 9.2 . 


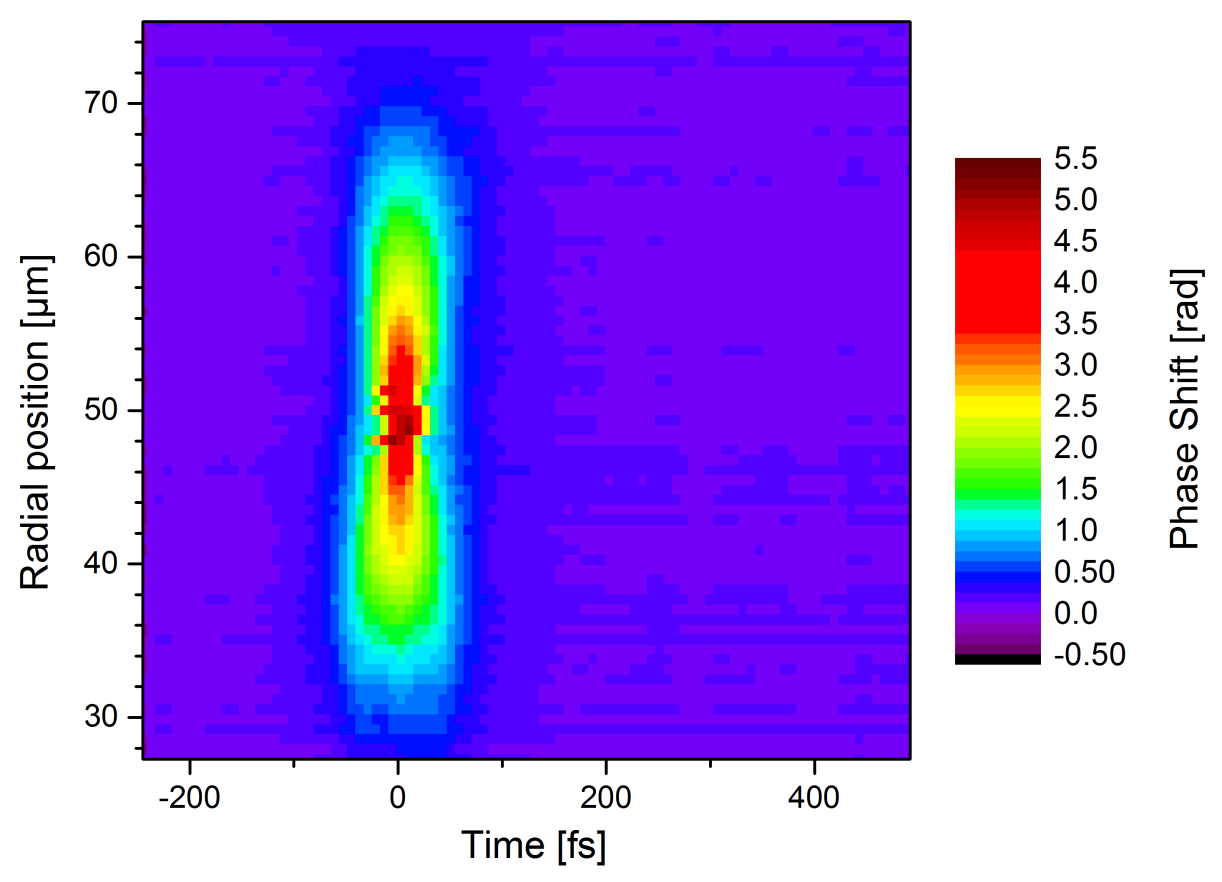

Figure 16.3.: Radially-resolved, time-dependent phase shift using a pump fluence of $0.5 \mathrm{Jcm}^{-2}$.

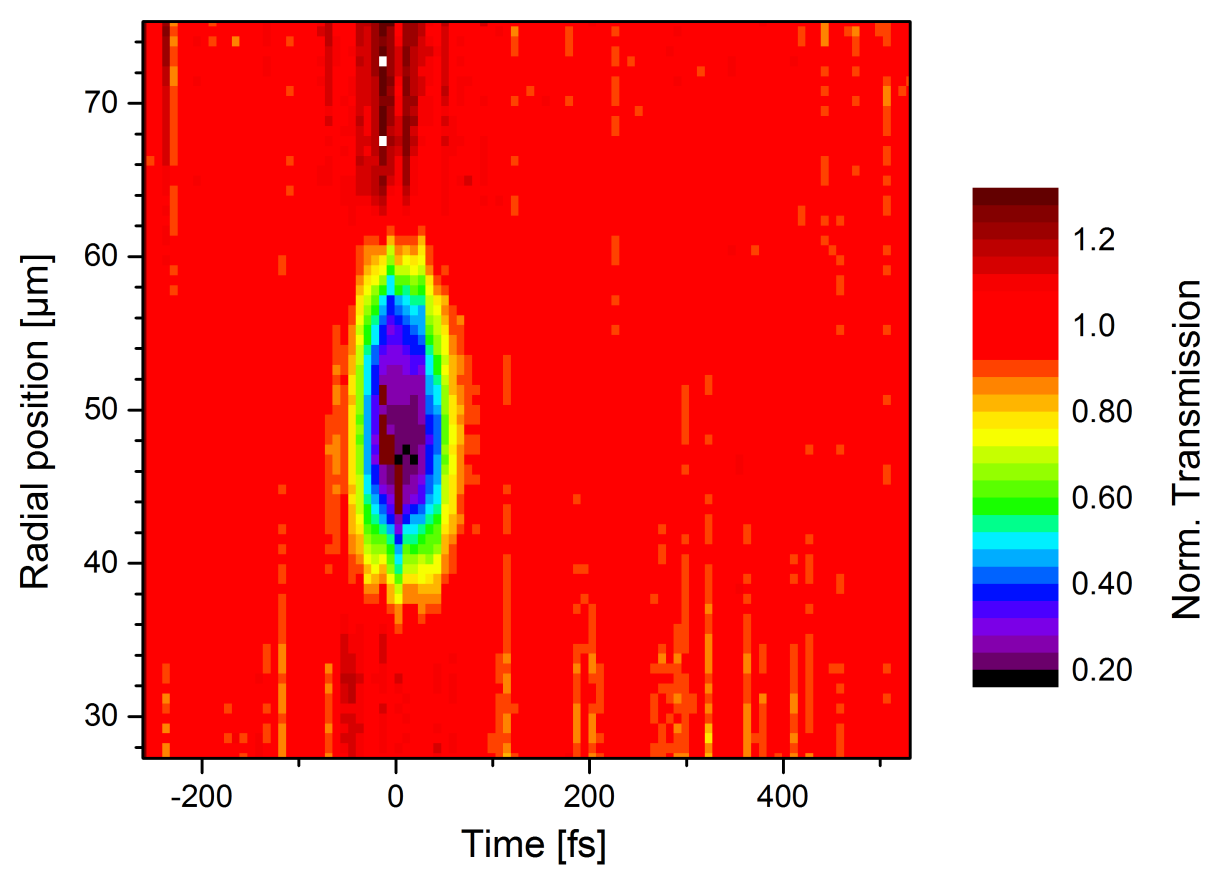

Figure 16.4.: Radially-resolved, time-dependent transmission using a pump fluence of $0.5 \mathrm{Jcm}^{-2}$. 
A further possibility is to create a double pulse utilizing the pulse shaper perform cross- and autocorrelation measurements, as done and described in detail in Götte et al. [13, 14]. Prior to each experiment we utilize the techniques described above obtain the pulse durations.

In figure 16.4, the same measurement as discussed above is presented, showing the transmission of the probe pulse. We observe that during the temporal overlap, the transmission strongly decreases to nearly 0 . Although this could be interpreted as absorption, it is more likely to be caused by the redistribution of light, as the pump pulse acts as a very strong lens causing light to be redistributed, visible in the CCD images in figure 9.2. In the SI measurements, this light redistribution is not as strong as in the pump-probe measurements, however it still can be seen that around time zero, the transmission is higher around the low transmission in the center.

\subsubsection{Delay scan at a moderate pump pulse fluence}

In figure $16.5 \mathrm{a}-\mathrm{b}$, radially-resolved pump-probe delay time dependent SI measurements at a moderate pump fluence are presented. Results obtained in the central part were discussed in part II In addition to the observations made discussed there, the full radially resolved measurement nicely visualizes the requirement of a high excitation, i.e. highly negative phase shift, for a high local transmission attributed to the LADIE effect.

\subsubsection{Pump pulse fluence dependence at different probe pulse fluences}

In part II] we discuss the possible mechanism of laser amplification in excited dielectrics. An important point is the nonlinearity of the amplification process. This assumption is based on the probe fluence dependent measurements. Both, global and local transmission in sapphire show a strong, nonlinear dependence on the incident probe pulse fluence. While the local dependence measured with the CCD camera is not sensitive to coherent emission only, we performed pump pulse fluence dependent spectral interference measurements (as shown in 9.4) using seven different probe pulse fluences. In the SI experiment, it is not simple to directly measure the pulse energy of reference- or probe pulse and therefore we measured the total power consisting of both pulses in a $1000 \mathrm{hz}$ mode prior to the respective measurement (thus the energy derived from this measurement is only an average value).

While in figure 9.6, the dependence is shown for two selected pump pulse fluences, in figure 16.6 an extended data set is presented. Each measurement consist of at least 100 different pump pulse fluence steps. The data presented is the transmission measured in the center of the excitation. At lower probe fluence the measurement range of pump fluence goes to approximately $2.2 \mathrm{Jcm}^{-2}$, being the pump fluence at which we observe the highest amplification of the probe pulse. At higher probe fluences we extend the measurement range to allow an observation of dynamics at high pump fluences.

In figure 16.6, the pump pulse peak fluence dependent transmission is shown for a selected range of probe fluences. At the lowest probe fluence, the transmission simply decreases with increasing pump fluence, eventually reaching zero. The dynamics are are very similar to the simulation shown in figure 10.3 . With increasing probe power, the dynamics change: 


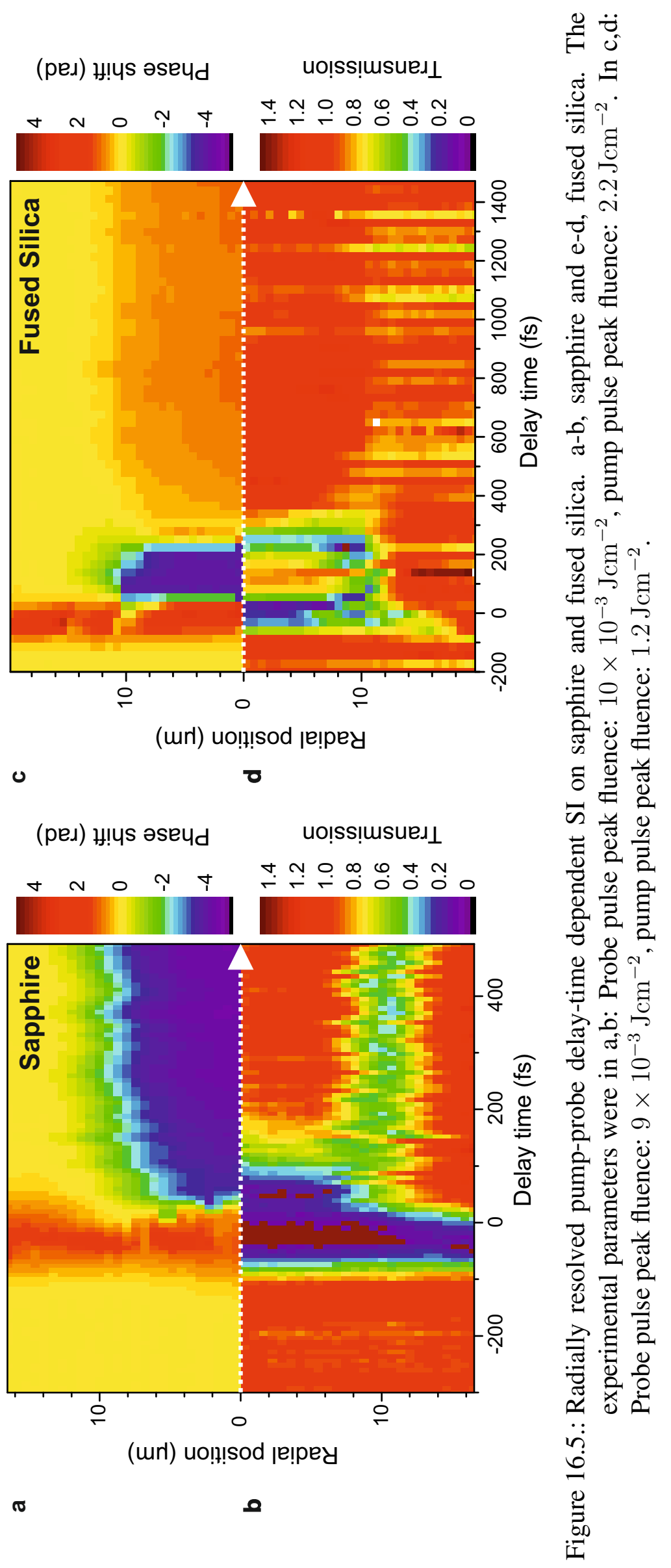




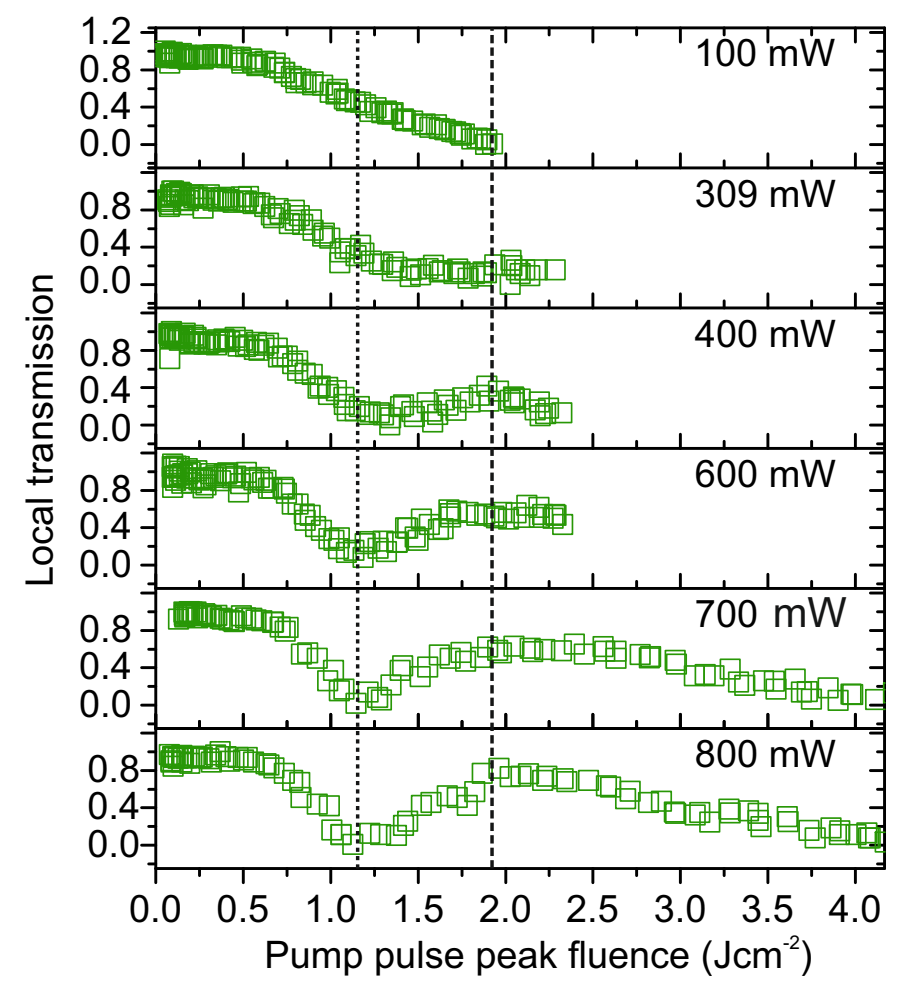

Figure 16.6.: Pump-pulse peak-fluence dependent local transmission under different probe pulse powers as indicated in the figures. Please note that the provided power is the total measured power consisting of probe and reference pulse, as this is a spectral interference measurement with initially identical reference- and probe pulse intensities. The dotted and dashed line represents the pump pulse fluence at which the probe fluence dependence shown in figure 9.6 is obtained from.

The transmission decreases much stronger for higher probe fluence, which we relate to the assumed three-photon absorption (see chapter 10). Increasing the probe pulse fluence even further, we observe that the transmission close to a pump fluence of $2 \mathrm{Jcm}^{-2}$ starts to increase due to the LADIE effect.

\subsubsection{Influence of pump pulse polarization}

In part [II we introduce a possible amplification process based on nonlinear stimulated emission. In such a process the polarization of the pump pulse should not play a role. In figure 16.7, CCD images are shown using a linear and a cicular polarization of the pump pulse under two different excitation conditions: absorption and amplification. As can be seen, both measurements provide identical results.

\subsubsection{Spectral-interference measurement under amplification conditions}

As promised in part $\Pi$, in this section a pump fluence dependent spectral interference measurement is provided that shows coherent amplification, i.e. a local transmission above 1. In figure 


\section{Absorption conditions}

\section{LADIE conditions}

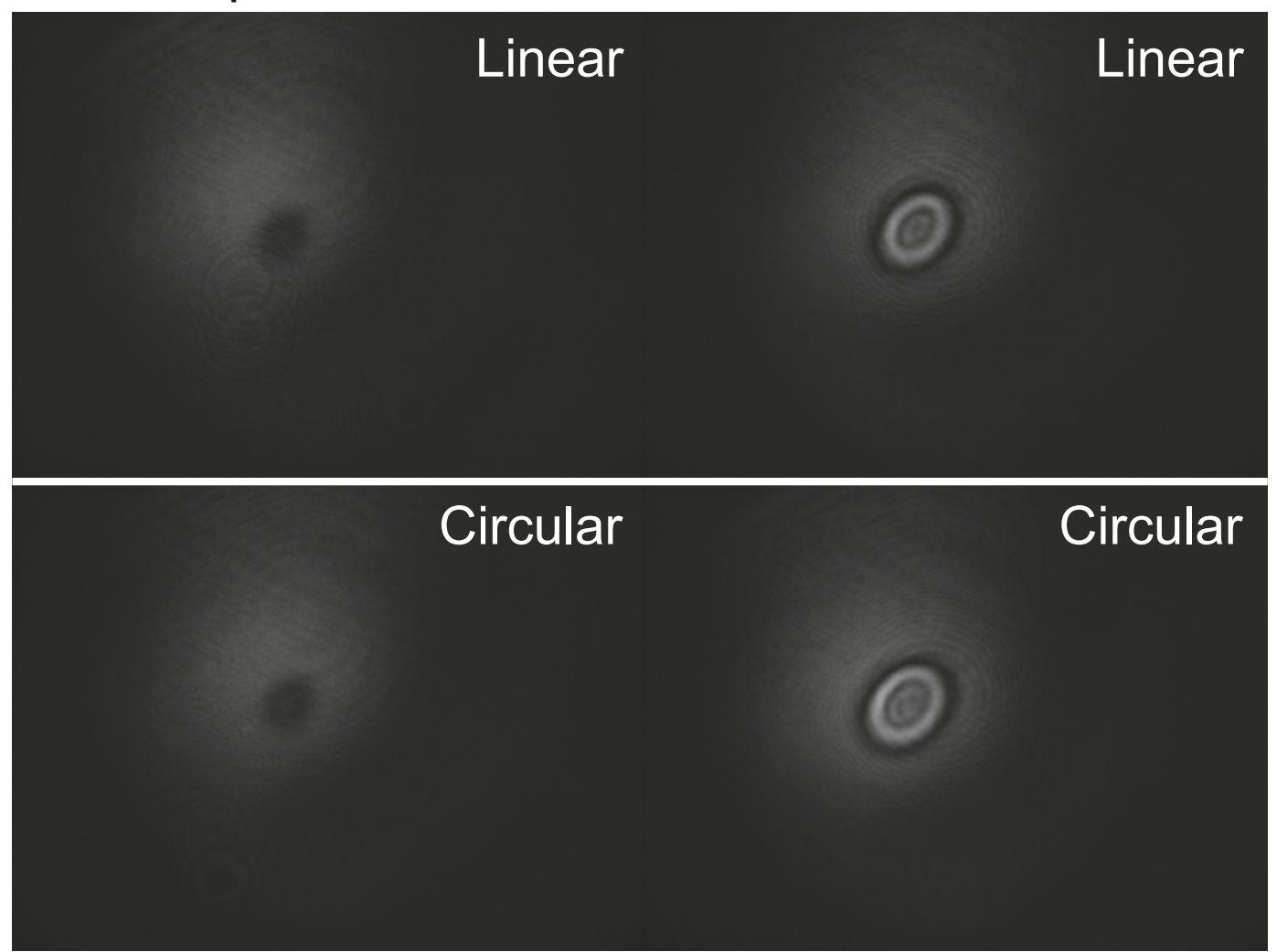

Figure 16.7.: CCD images of a $400 \mathrm{~nm}$ probe pulse, $300 \mathrm{fs}$ after excitation using different pump conditions: absorption and amplification (LADIE). The polarization state of the pump pulse is indicated within the images. 


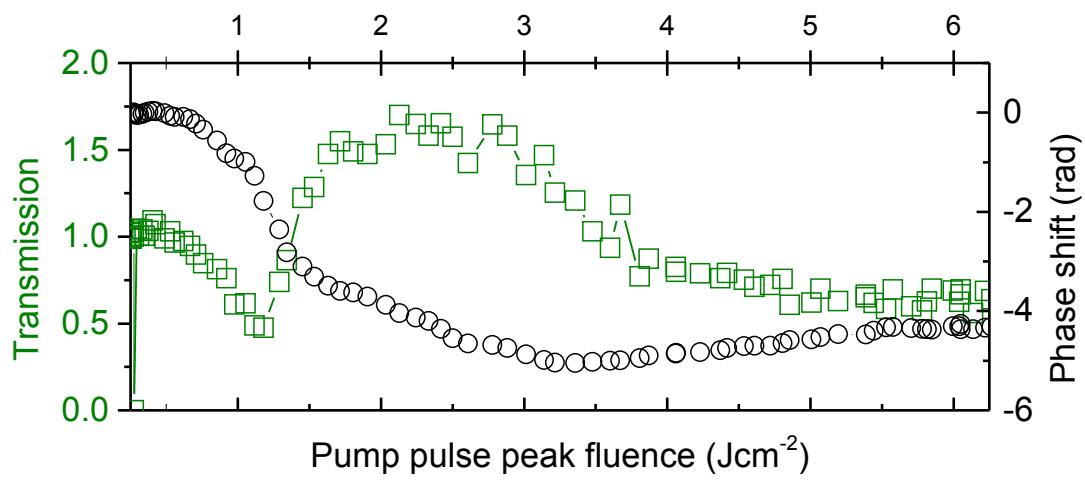

Figure 16.8.: Pump pulse peak fluence dependent local transmission (green squares) and phase shift (black circles) measured in the SI configuration in the center of excitation.

16.8, local transmission (green squares) and corresponding phase shift (black circles) is shown in dependence on the incident pump pulse fluence. While this measurement is mostly identical to the one presented in section 9.2 , it was taken when the probe pulse was intentionally made much weaker in comparison to the reference pulse. In such a case, it is possible to measure a normalized ${ }^{3}$ local transmission above one (see section 7.2). We observe, that all dynamics are nearly identical to the ones in figure 9.2. However, we observe that the initial minimum is not as pronounced compared to the other intensity scans. The total energy of probe and pump pulse was measured to be $1.2 \mathrm{~mW}$, which is higher compared to the measurements shown in section 16.1.4 explaining why we are observing a transmission above one.

\footnotetext{
${ }^{3}$ Normalized to the measured transmission when no pump pulse was incident.
} 


\subsection{Fused silica}

\subsubsection{Delay scan under LADIE conditions}

In figure 16.5 -d results of a delay scan on fused silica at a moderate pump fluence of $1.2 \mathrm{Jcm}^{-2}$ are presented. Results obtained in the central part, indicated by the white dashed arrow, were discussed in part $[\mathrm{I}$. Here we present the full radially-resolved data.

The LADIE effect can easily be identified in this spatially-resolved measurement: When the phase shift is highly negative, the LADIE effect, identified by a high transmission, is most pronounced. Thus, the increasing strength of excitation must clearly be related to a certain number of conduction band electrons, as discussed in part III.

\subsubsection{Delay scans at different pump pulse fluences}

We repeated the delay scan under different pump-pulse-peak-fluences to find out if it has an influence on the temporal and spatial distribution of phase shift and transmission. In figure 16.9, three measurements using $1.2 \mathrm{Jcm}^{-2}, 2.6 \mathrm{Jcm}^{-2}$ and $3.2 \mathrm{Jcm}^{-2}$ pump pulse peak fluence were used. While the first measurement is the one already discussed in the last subsection, the other two, obtained at much higher pump fluences, will be discussed next:

\section{Phase shift}

At first, one can notice that very close to time zero, when the OKE is strongest, the phase shift does not have a nice radial distribution as expected, like e.g. shown in figure 16.3 . The reason can be found in the evaluation of the measurement data. The problem we encounter is that at high levels of excitation, the contributions by the OKE and the conduction band electrons can overlap:

Under such conditions, the probe pulse can carry both information (negative phase shift by electrons and positive by $\mathrm{OKE}$ ) and sometimes the fringes of the interfering spectrum (see 7.2) perform phase jumps: This happens when the phase shift rapidly decreases from e.g. +4 to -4 rad within one measurement step (usually a few femtoseconds). Thus it "looks" for the measurement program as e.g. the phase decreases, whereas it actually increases. This can be accounted for manually, by multiplying the values by $\pm 2 \pi$. This is time consuming as every radial position has to be corrected by hand. As we are not interested in the Kerr dynamics at very high pump fluences, it has not been done for all measurements/radial position. We do not observe this phenomenon to that extent in sapphire. The reason is that in sapphire we have used a smaller delay time step size such that the rapid changes can be followed and evaluated more easily. In future experiments, a smaller step size should be used for fused silica as well to avoid the discussed problem.

When we look at the phase shift distribution at the three different pump-pulse-peak-fluences, we do not see a big difference: As expected, a higher pump fluence will lead to a stronger negative phase shift at early delay times and a higher positive phase shift at late delay times. This is further discussed in the next section where we investigate the pump fluence dependence at different delay times. It might sound unnecessary or redundant to perform delay scans at different pump fluences 

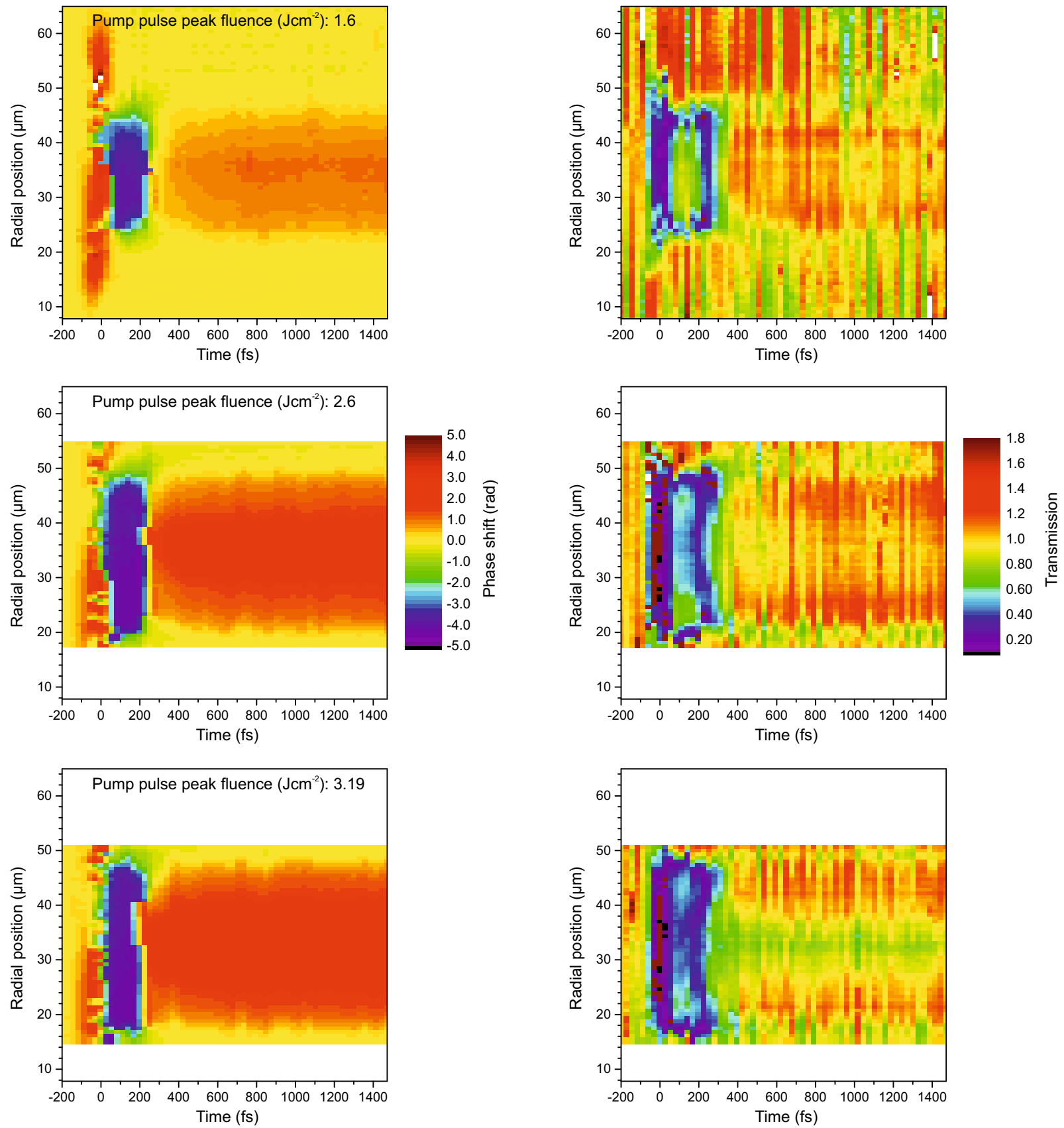

Figure 16.9.: Radially-resolved, pump-probe delay-time dependent SI measurement on fused silica under different pump-pulse-peak-fluence conditions as indicated in the figures. On the left side is the phase shift shown, on the right the corresponding transmission distribution. 
and pump-fluence scans at different delay times, however sometimes one scan can resolve not all the dynamics, as we will see later.

\section{Transmission}

At first, we notice that the transmission is relatively noisy compared to the very smooth phase shift: Although pulse-to-pulse energy fluctuations do not influence the transmission measurement, small temperature fluctuations in the birefringent crystals have a strong influence on the respective refractive indices of the ordinary and extraordinary axis. A possible upgrade could be a thermal stabilization of the BBO crystals, as done in other spectral interference measurements [112] utilizing the same technique for the creation of a double pulse (see 7). The noise is much lower in the center of the investigated area, which can be related to a higher local probe fluence.

The dynamics using the lowest pump pulse peak fluence, as discussed in the main part, show that after the increase of the phase shift by the OKE, the transmission drops when the phase shift decreases due to conduction band electrons. Within the typical low transmission during the presence of conduction band electrons, we observe the short time in which LADIE conditions are fulfilled and the transmission has a maximum. Then, due to the trapping of electrons, their density decreases leading to an initially decreasing transmission followed by an increase to unity when only self trapped excitons are present. This is seen in detail in figure 16.10, showing the dynamics in the center. From the radially resolved measurement in figure 16.9, we notice that at higher radii (24 and $44 \mu \mathrm{m}$ ), where the fluence is too low for LADIE conditions to be fulfilled, no increase in transmission is observed. However, there are some small changes in transmission even at long delay times. This might be related to beam propagation effects, as discussed later. Important to note is that in this pump fluence case, the increase in transmission at longer delay times due to the self-trapping process happen at all radial positions at the same time.

This is very different for a higher pump fluence: Here, we observe that in the center, where the fluence is highest, the trapping dynamics seem to be faster compared to lower excitation conditions at larger radii. This can be seen in more detail in figure 16.10. However, the phase shift seem to reach the final value quicker than the transmission providing a slightly different dynamic. The reason can be found in the earlier excitation of electrons and thus a trapping process that starts consequentially earlier as well.

Using the highest pump fluence of $3.2 \mathrm{Jcm}^{-2}$, the transmission, shown in figures 16.9 and 16.10 . does not reach a value of 1 at high delay times. We relate this to the survival of conduction band electrons that do not get trapped. Although their contribution to the phase shift might only have a minor impact, they can strongly affect the transmission, as the STE's usually do not change the transmission of the probe pulse (see chapter 2 and 4). What can also be observed, especially in the data taken from the center, is that when the trapping sets in and the phase shift increases, the transmission does not decrease as much for high pump fluences as compared to the case when only a pump fluence of $1.6 \mathrm{Jcm}^{-2}$ was used. Here the transmission nearly resembles a "W" like shape. The reason for the reduced drop in transmission might be related to the changed temporal dynamics and need to be investigated. 

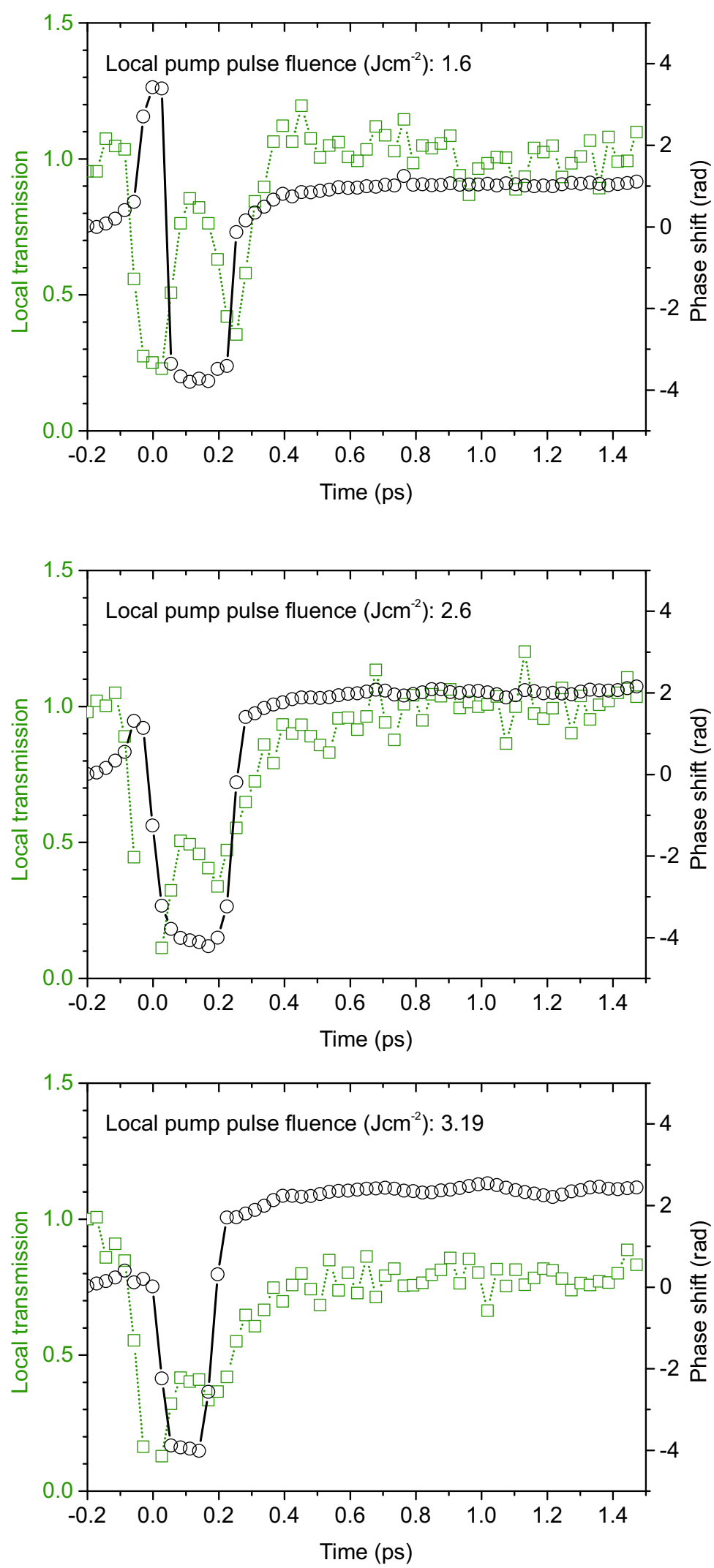

Figure 16.10.: Pump-probe delay-time dependent phase shift and transmission in fused silica under different pump pulse fluence conditions as indicated in the figures. 


\subsubsection{Intensity scan}

Similar to the delay scan in part $\Pi$, only the pump fluence depended dynamics of the excitation taken from the center of a radially-resolved measurement were discussed. The fully resolved data of the measurement is shown in figure 16.11. As this measurement was taken at the time (approx $180 \mathrm{fs}$ ) when the contribution by conduction band electrons were present, we obtain a scan similar to the one in sapphire, shown in figure 9.3. The enhanced transmission is following a specific strength of excitation and thus the local pump fluence, as expected.

However, different then sapphire, it seems that the changes in the optical properties, phase shift an transmission, seize to follow the local pump fluence already at $1.9 \mathrm{Jcm}^{-2}$, which is below the observed ablation threshold of $2.5 \mathrm{~J} / \mathrm{cm}^{2}$. This is further discussed in the next section with respect to propagation effects.

When the same scan is repeated at a much later delay time of $1500 \mathrm{fs}$, we obtain a completely different picture. In figure 16.12, the phase shift is shown. We observe that with increasing fluence the phase shift starts to increase up to a maximum value of $2.8 \mathrm{rad}$. The positive phase shift is accounted to the self-trapped excitons created by the ever increasing number of electrons that get trapped (see chapter 2).

Up to this point, the observed dynamics were just as expected. However, at high fluences, above $2 \mathrm{Jcm}^{-2}$, we observe that at large radii a negative phase shift appears. As a negative phase shift is usually associated with the creation of conduction band electrons, the only possibility to measure their signature in fused silica at such long delay times (longer than the trapping time of $150 \mathrm{fs}$ ) is that some CB electrons must have "survived" the electron tapping process. This is possible when the number of promoted electrons exceeds the number of possible trapping states available, which is usually assumed to be one for each $\mathrm{SiO} 2$ complex [22,44].

The question that directly arises here is why the negative phase shift is detected at high radial positions, where the local pump fluence is relatively low and not intense enough to create an excessive amount of electrons:

Comparing the phase shift to the corresponding transmission data, shown in figure 16.13 , we notice that when the material is excited with at least $2 \mathrm{Jcm}^{-2}$, the transmission starts to decrease in the center (similar to the delay scan discussed above). This behavior continuous with increasing fluence and therefore the onset of the decreasing transmission is directly correlated to the onset of negative phase shift at large radial position. Thus, it might be possible that the excessive conduction band electrons located in the center might lead to a plasma defocusing of the probe pulse such that the negative phase shift appears at high radial positions. This would coincide, as light that was original in the center is diverted towards larger radii leads to an additional decrease in transmission (in addition to normal free carrier absorption).

However, a real qualitative and quantitative analysis is not possible without having a proper simulation model that takes linear and nonlinear propagation effects into account. 


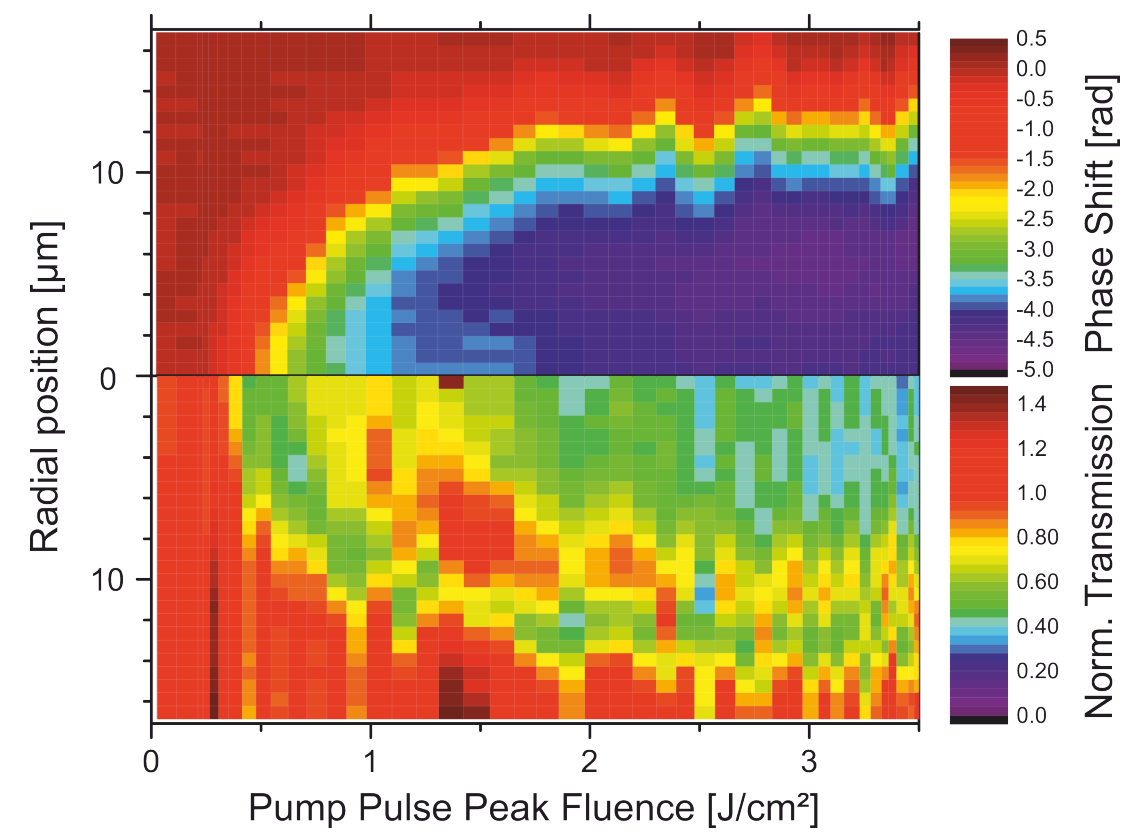

Figure 16.11.: Radially-resolved phase shift (a) and transmission (b) in dependence on the applied pump pulse peak fluences at a time step of around $180 \mathrm{fs}$ after excitation.

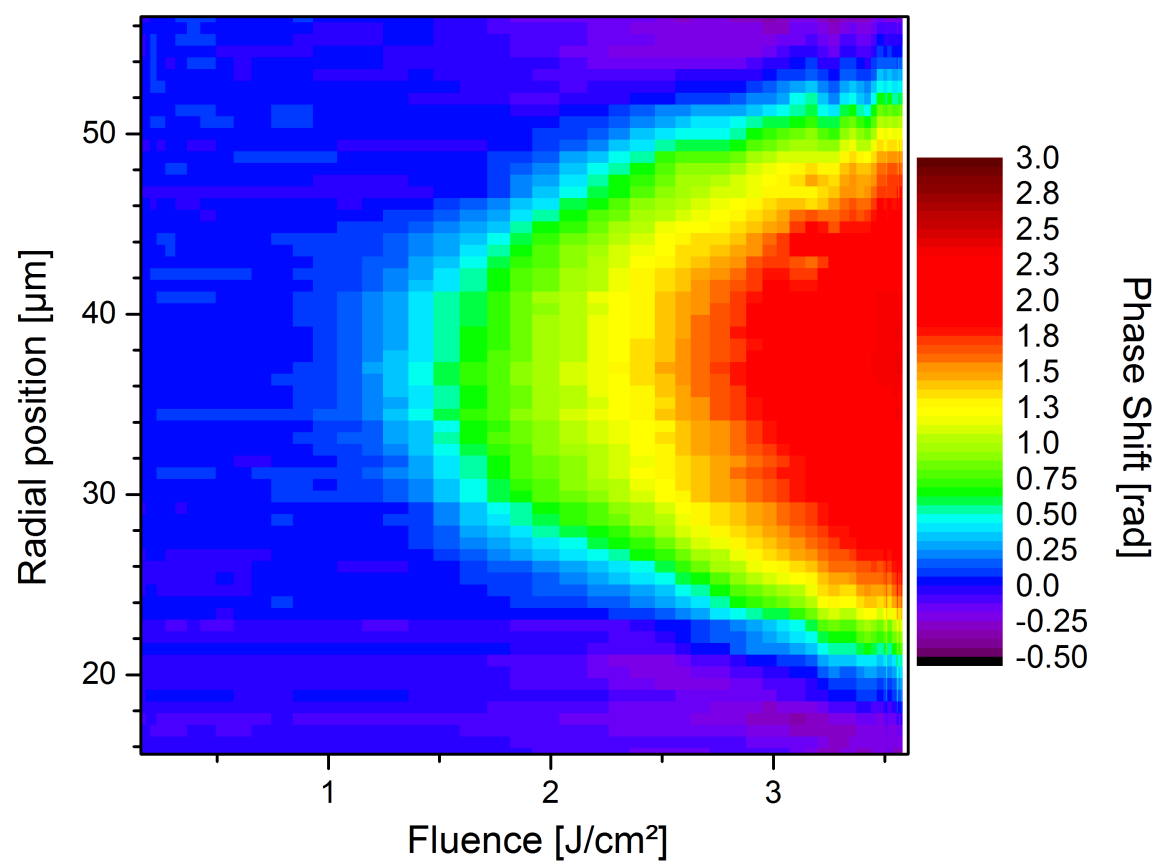

Figure 16.12.: Radially-resolved phase shift in dependence on the applied pump pulse peak fluences at a delay time of around $1500 \mathrm{fs}$ after excitation. 


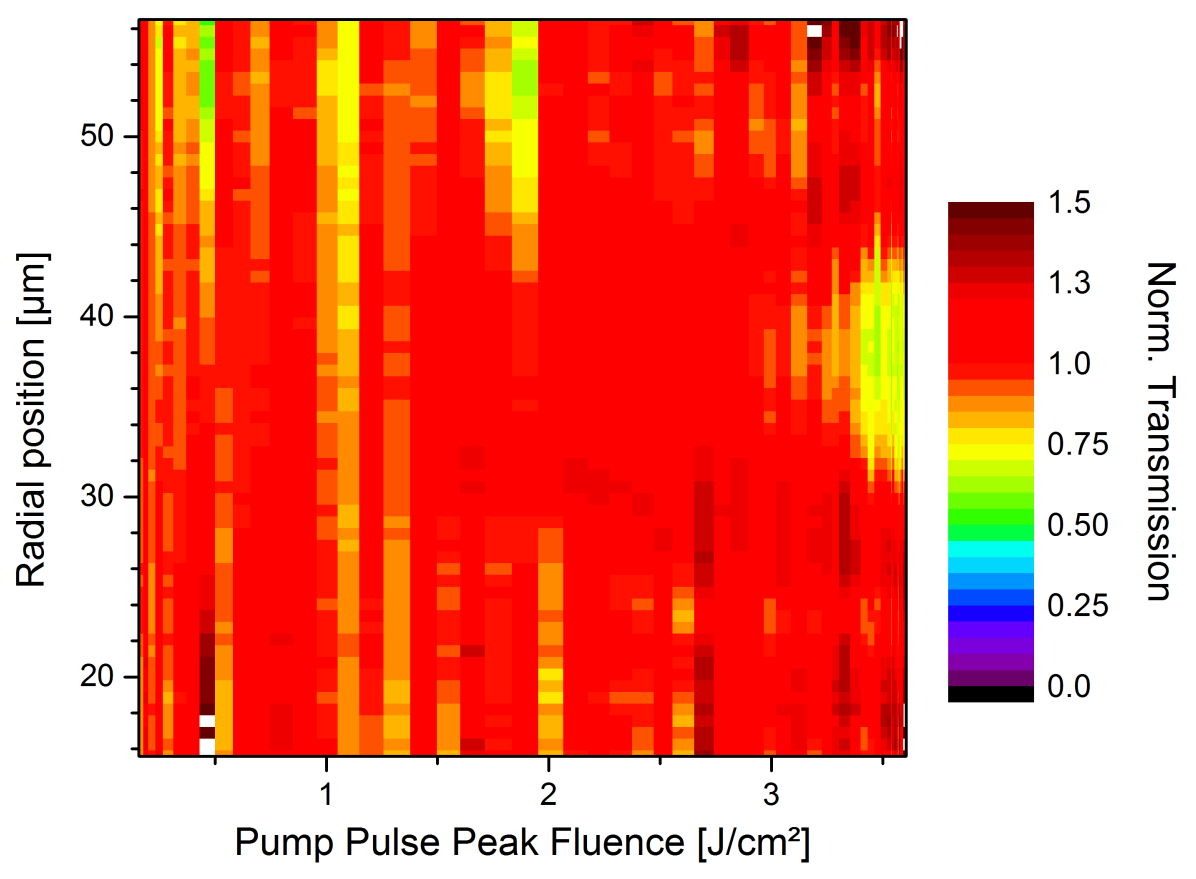

Figure 16.13.: Radially-resolved transmission in dependence on the applied pump pulse peak fluences at a delay time of around $1500 \mathrm{fs}$ after excitation.

\subsection{Propagation effects}

In the pump-fluence dependent measurements, shown in figure 9.4, we observe that the changes in phase shift and transmission follow the Gaussian beam profile and therefore the local pump fluence of the pump pulse. However, from a certain peak fluence, this is no longer true and we relate this to propagation effects. It is possible that pump, probe or both pulses might be affected by those (see section 4.5). To estimate a lower limit of the pump fluence, at which the "real" pump fluence dependence is affected, we compare the dynamics obtained from the center of the pulse while increasing the pump pulse peak-fluence step by step to the dependence obtained from the radial distribution (Gaussian), obtained at a single pump fluence setting (Similar to what as done for water (see section 13.3). It is assumed that the on axis beam (at $r=0 \mu \mathrm{m}$ ) in the center is not affected by these effects:

\section{Sapphire}

In figure 16.14, such a comparison for sapphire at a delay time of $300 \mathrm{fs}$ is shown (extracted from figure 9.4). We observe that for a pump fluence below $2.5 \mathrm{Jcm}^{-2}$ the slopes are identical. Even at a fluence that is around the ablation threshold $\left(3.75 \mathrm{Jcm}^{-2}\right)$, the slopes are still similar and an extraction of the qualitative and even quantitative pump fluence dependence is valid. However, we can see that the dynamics are shifted towards lower local pump fluences. A proper explanation and understanding can only be achieved by a more complex simulation including a full propagation aspect of both, pump and probe. 


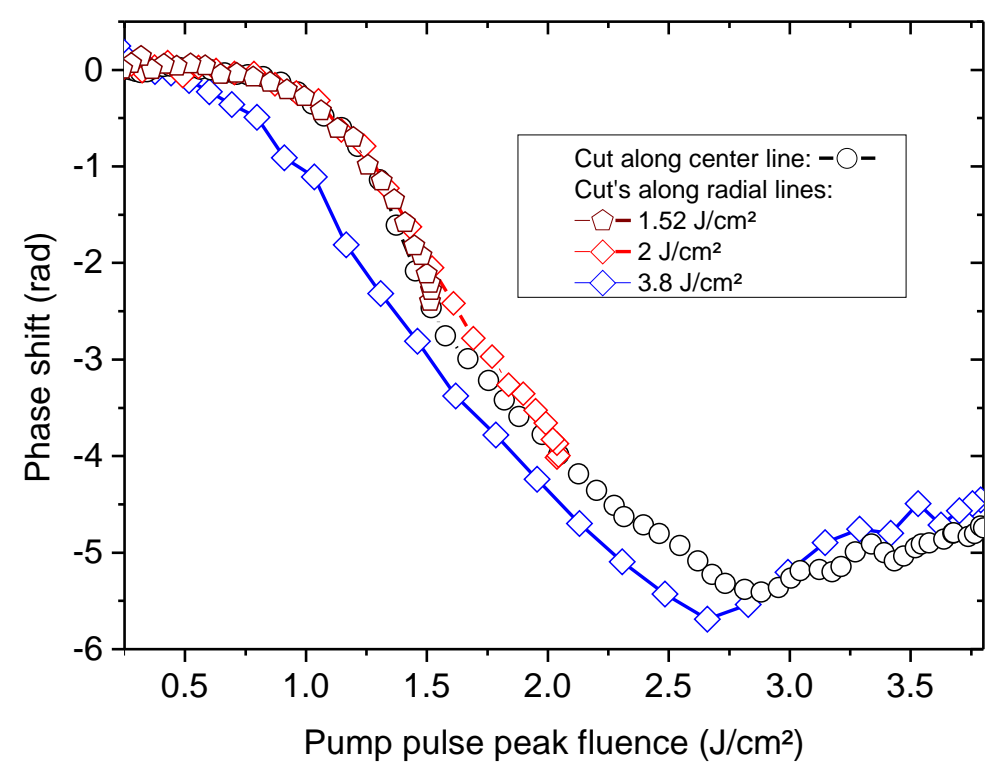

Figure 16.14.: Pump fluences dependence of the phase shift in sapphire extracted from the central part and from the radial fluence distribution at different pump fluences. Data was obtained at a delay time of $300 \mathrm{fs}$.

The most important observation is, that at a fluence of $2 \mathrm{Jcm}^{-2}$, where we observe the strongest amplification (described in part II), central and radial distribution are equal, supporting the assumption that propagation cannot be the responsible mechanism for the observed coherent amplification of the probe pulse.

\section{Fused silica}

In fused silica, we also compared the central vs radial fluences dependence. However, we did it at two different pump-probe delay times to account for the distribution by conduction band electrons and self-trapped excitons:

In figure 16.15, the comparison is shown at a delay time of approximately $180 \mathrm{fs}$, a time step at which the contribution by conduction band electrons to the optical properties is assumed to be largest. We observe that below $1 \mathrm{Jcm}^{-2}$ the slopes are nearly identical. This fluence is identical to the one at which amplification was observed in the center. This shows that the LADIE effect occurs before the onset of propagation effects.

At larger fluences, the distributions largely varies by requiring higher pump fluences to reach a certain negative phase shift. An interpretation is at this point very difficult as an increasing pump fluence can also influence the temporal dynamics, as seen in the last section: The higher the pump fluence, the earlier electrons are created by the pump pulse and the earlier the trapping process starts.

When the same comparison is done at much later delay time of $1500 \mathrm{fs}$, shown in figure 16.16, a very different behavior is measured: At this time step, as discussed earlier, the contribution by self trapped excitons is dominant. Up to $2 \mathrm{Jcm}^{-2}$ the distributions are identical. For a very high pump 


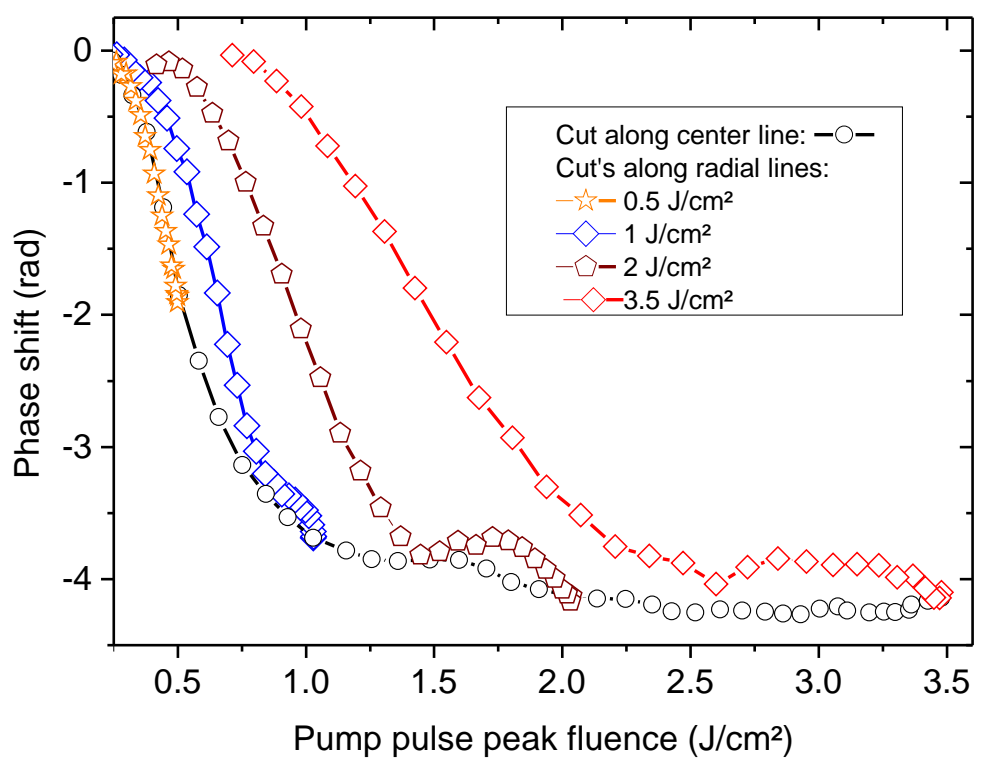

Figure 16.15.: Pump pulse peak fluence dependence of the phase shift in fused silica extracted from the central part and from the radial fluence distribution at different pump fluences. Data was obtained at a delay time of $180 \mathrm{fs}$ where the contribution by electrons is assumed to be dominant.

pulse fluence setting, the distribution is very different, qualitatively and quantitatively. We relate to the "survival" of conduction band electrons and strong propagation effects.

\subsubsection{Influence of beam profiles}

At this point, we want to discuss how the ratio of beam sizes (pump vs. probe) influence the measured transmission. To that extent we will qualitatively compare three measurements in which we used probe beam diameters that were either larger than the pump beam or comparable in size.

We found that all dynamics, temporal and fluence wise, are identical, no matter which beam sizes were used. However, we notice that when comparable beam sizes (see figure $16.17 \mathrm{p}$ and c) are used, a large amount of light is scattered or distributed towards large radii when the conduction band electron plasma was created. This effect is nearly not noticeable when a probe beam was used much larger than the pump, as seen in figure 16.17 a. Please note that in b and c, "ghost images" are visible (replica of the original image). These are produced in a thick OD filter in front of the camera. 


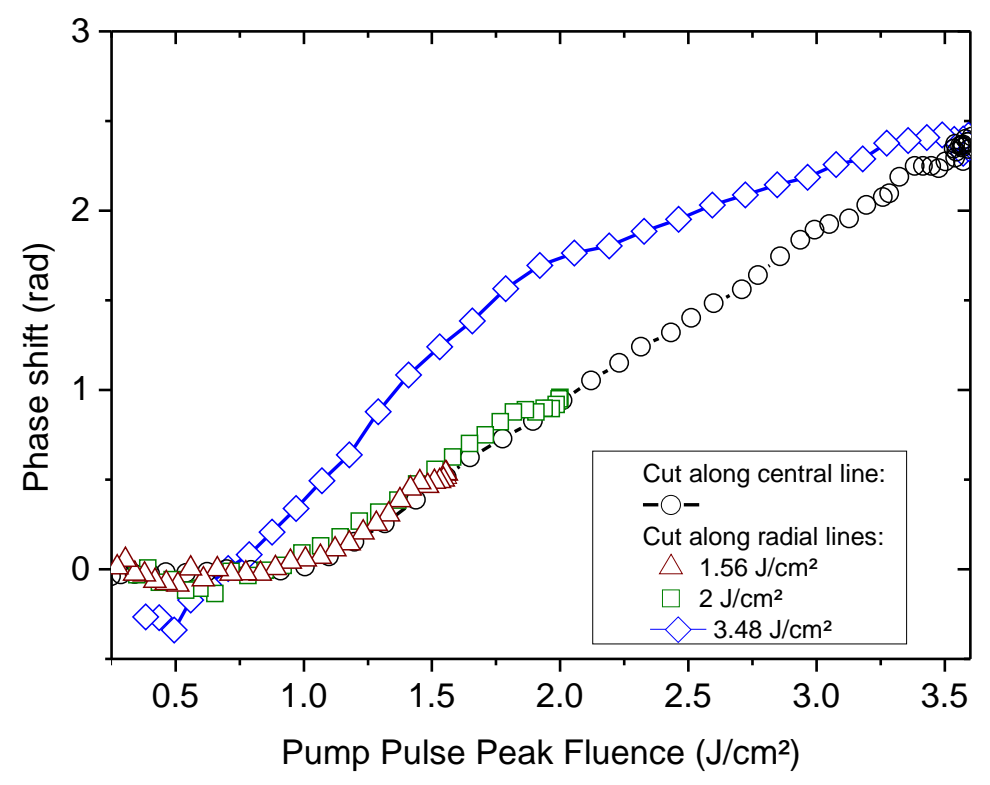

Figure 16.16.: Pump fluences dependence of the phase shift in fused silica extracted from the central part and from the radial fluence distribution at different pump fluences. Data was obtained at a delay time of $1500 \mathrm{fs}$ where the contribution by self trapped excitons is dominant.

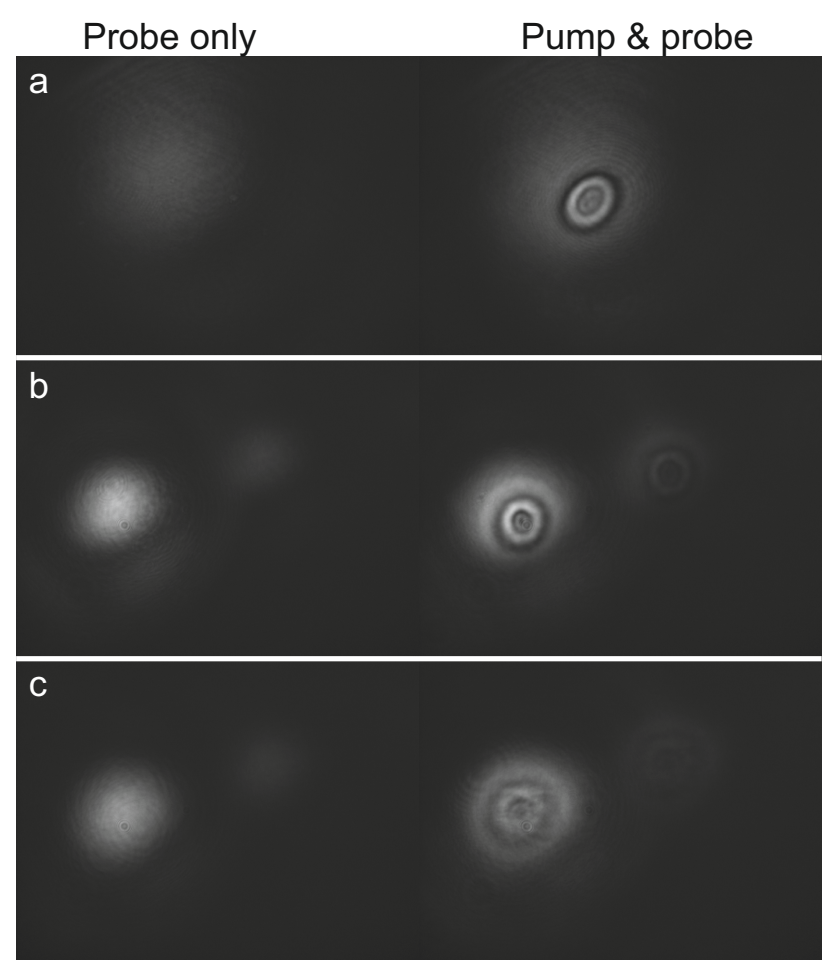

Figure 16.17.: CCD images using different sized probe and pump pulses under similar pump conditions. Beam width ( $1 / e^{2}$ beam radius) are as follows: Pump pulse: $19.3 \mu \mathrm{m}$ in a, $21.8 \mu \mathrm{m}$ in $\mathrm{b}, 22.1 \mu \mathrm{m}$ in c. Probe pulse: $51.1 \mu \mathrm{m}$ in a, $33.9 \mu \mathrm{m}$ in $\mathrm{b}, 33.9 \mu \mathrm{m}$ in c. 


\section{Location of used measurement and evaluation software and experimental data}

The measurements were done by using different LabView 8 and 12 programs that are located on the group network drive in "...\Lab-View-8 $\backslash$ Plasma Mirror $\backslash$ DataAcquisition $\backslash$ "and"... $\backslash$ Lab-View$12 \backslash$ Spectral-Interference $\backslash$ DataAcquisition $\backslash "$. The detailed description of how to perform a measurement can be found in the same folder on the network drive.

The evaluation of the raw-data was partly done in LabView 8 and in Mathematica in different programs. All can be found on the group network in the folders "... \Lab-View-8 $\backslash$ Plasma Mirror $\backslash$ Data Analysis $\backslash$ sarpe $\backslash$ " and "... $\backslash$ Lab-View-8 $\ \backslash$ Plasma Mirror $\backslash$ Data Analysis $\backslash$ Mathematica $\backslash$ ". A detailed description of how to evaluate the data can be found there or within the programs as well.

All data recorded during the $\mathrm{PhD}$ time is located in the folders of the measurement date under "...।Projects \water plasma \". The data used for the publications is listed in the following tables.

\subsection{Overview of measurements for part II}




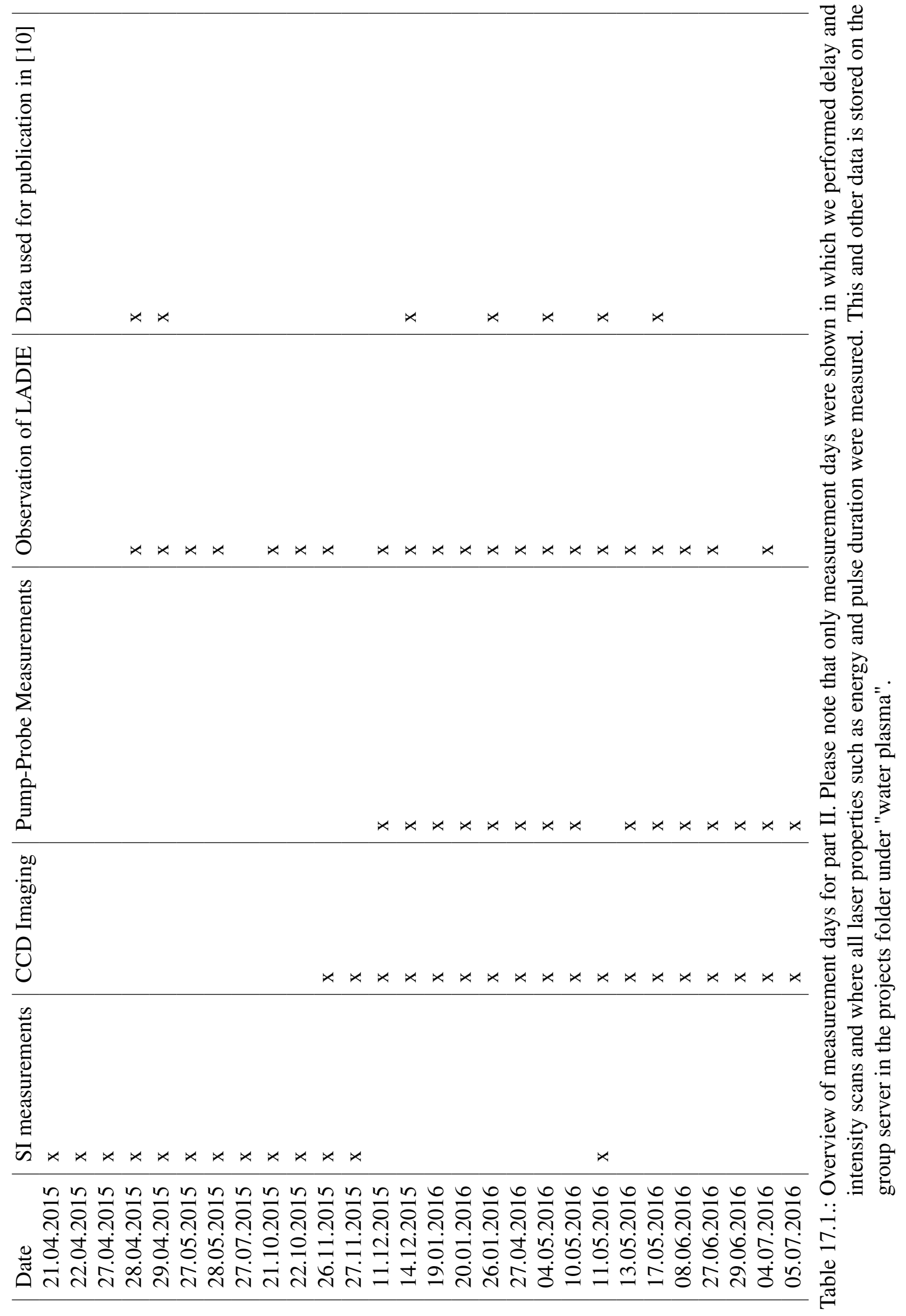




\subsection{Overview of measurements for part III}

\begin{tabular}{l|l|} 
Date & Data used for publication in [12] \\
10.02 .2015 & \\
11.02 .2015 & \\
20.02 .2015 & \\
23.02 .2015 & \\
03.03 .2015 & \\
02.04 .2015 & \\
21.04 .2015 & \\
22.04 .2015 & \\
28.04 .2015 & \\
29.04 .2015 & \\
22.05 .2015 & $\mathrm{x}$
\end{tabular}

Table 17.2.: Overview of measurement days for part III. Please note that only measurement days were shown in which we performed delay and intensity scans and where all laser properties such as energy and pulse duration were measured. 


\section{Overview of the most important symbols}

\begin{tabular}{|c|c|c|}
\hline Symbol & Physical quantity & SI-Unit \\
\hline$c$ & Vacuum speed of light & $\mathrm{m} / \mathrm{s}$ \\
\hline$\varepsilon_{0}$ & Vacuum permittivity & $\mathrm{A}^{2} \mathrm{~s}^{4} \mathrm{~kg}^{-1} \mathrm{~m}^{-3}$ \\
\hline$\varepsilon_{\mathrm{r}}$ & Relative permittivity & - \\
\hline$\varepsilon_{\mathrm{b}}$ & Permittivity of the unexcited material & $\mathrm{A}^{2} \mathrm{~s}^{4} \mathrm{~kg}^{-1} \mathrm{~m}^{-3}$ \\
\hline$e$ & Elementary charge & $\mathrm{C}$ \\
\hline$T$ & Temperature & $\mathrm{K}$ \\
\hline$m_{e}$ & Vacuum electron mass & $\mathrm{kg}$ \\
\hline$m_{\mathrm{c}}$ & Conduction band electron mass & $\mathrm{kg}$ \\
\hline$m_{\mathrm{v}}$ & Valence band electron mass & $\mathrm{kg}$ \\
\hline$E$ & Electric field amplitude & $\mathrm{V} / \mathrm{m}^{2}$ \\
\hline$I$ & Cycle-averaged intensity & $\mathrm{W} / \mathrm{m}^{2}$ \\
\hline$I_{0}$ & Cycle-averaged peak intensity & $\mathrm{W} / \mathrm{m}^{2}$ \\
\hline$w_{0}$ & $1 / e^{2}$ Beam radius & $\mathrm{m}$ \\
\hline$z_{0}$ & Rayleigh range & $\mathrm{m}$ \\
\hline$\Delta t$ & Full width half maximum pulse duration & $\mathrm{s}$ \\
\hline$\phi_{3}$ & Third order temporal phase of the laser pulse & $\mathrm{fs}^{3}$ \\
\hline$R$ & Reflection coefficient (regarding to the intensity) & - \\
\hline$\omega_{l}$ & Laser angular frequency & $1 / \mathrm{s}$ \\
\hline$\alpha$ & Absorption coefficient & \\
\hline$\omega_{\mathrm{Pr}}$ & Probe pulse angular frequency & $1 / \mathrm{s}$ \\
\hline$\omega_{\mathrm{P}}$ & Pump pulse angular frequency & $1 / \mathrm{s}$ \\
\hline$E_{\mathrm{P}}$ & Pulse energy & $\mathrm{eV}$ \\
\hline$E_{\mathrm{Ph}}$ & Photon energy & $\mathrm{eV}$ \\
\hline$E_{\mathrm{G}}$ & Band gap between valence and conduction band & $\mathrm{eV}$ \\
\hline$\mu_{e / h}$ & Chemical potential of electrons or holes & $\mathrm{eV}$ \\
\hline$E_{\text {Ponderomotive }}$ & Ponderomotive energy shift & $\mathrm{eV}$ \\
\hline$E_{\text {Crit }}$ & Critical energy for impact ionization & $\mathrm{eV}$ \\
\hline$E_{\mathrm{T}}$ & Transition/Transmission energy & $\mathrm{eV}$ \\
\hline$\tilde{E}_{\mathrm{G}}$ & Corrected band gap & $\mathrm{eV}$ \\
\hline$\gamma$ & Keldysh parameter & - \\
\hline$f_{e / h}$ & Fermi function of electrons (e) or holes (h) & - \\
\hline$\Delta f$ & Difference of Fermi function & - \\
\hline$\varepsilon$ & Complex dielectric function & $\mathrm{A}^{2} \mathrm{~s}^{4} \mathrm{~kg}^{-1} \mathrm{~m}^{-3}$ \\
\hline$\tilde{n}$ & Complex refractive index & - \\
\hline
\end{tabular}




\begin{tabular}{|l|l|l|}
$\Delta \tilde{n}$ & Change of the complex refractive index & - \\
$n_{\mathrm{r}}$ & Real part of the complex refractive index & - \\
$n_{\mathrm{i}}$ & Imaginary part of the complex refractive index & - \\
$\tilde{n}_{\mathrm{Dr}}$ & Drude part of the complex refractive index & - \\
$\beta_{\mathrm{CM}}$ & Clausius-Mossotti coefficient & - \\
$t$ & Time & $\mathrm{s}$ \\
$t_{\mathrm{ret}}$ & Retarded time & $\mathrm{s}$ \\
$\tau$ & Electron scattering time coefficient & $\mathrm{s}$ \\
$\tau^{\star}$ & Scattering time coefficient & $\mathrm{s}$ \\
$\tau_{C-C}$ & Carrier-Carrier scattering time & $\mathrm{s}$ \\
$\tau_{C-P}$ & Carrier-phonon scattering time & $\mathrm{s}$ \\
\hline & Order of multi-photon ionization process (nr. of photons) & - \\
$n$ & Multi-photon ionization coefficient & $\mathrm{s}^{-1} \mathrm{~m}^{(2 \mathrm{n}-3)} \mathrm{W}^{-n}$ \\
$\sigma_{n}$ & Avalanche ionization coefficient & $\mathrm{s}^{-1} \mathrm{~W}^{-1} \mathrm{~m}^{-5}$ \\
$\alpha_{\mathrm{AI}}$ & conduction-band-electron density & $\mathrm{m}^{-3}$ \\
$N$ & Critical electron density & $\mathrm{m}^{-3}$ \\
$N_{\mathrm{C}}$ & Maximum valence band electron density & $\mathrm{m}^{-3}$ \\
$N_{\mathrm{Max}}$ & Plasma frequency & $1 / \mathrm{s}$ \\
$\omega_{\mathrm{Pl}}$ & & \\
\hline
\end{tabular}

Table 18.1.: Used symbols and quantities. 


\section{Quantities for the calculation of laser excitation of sapphire}




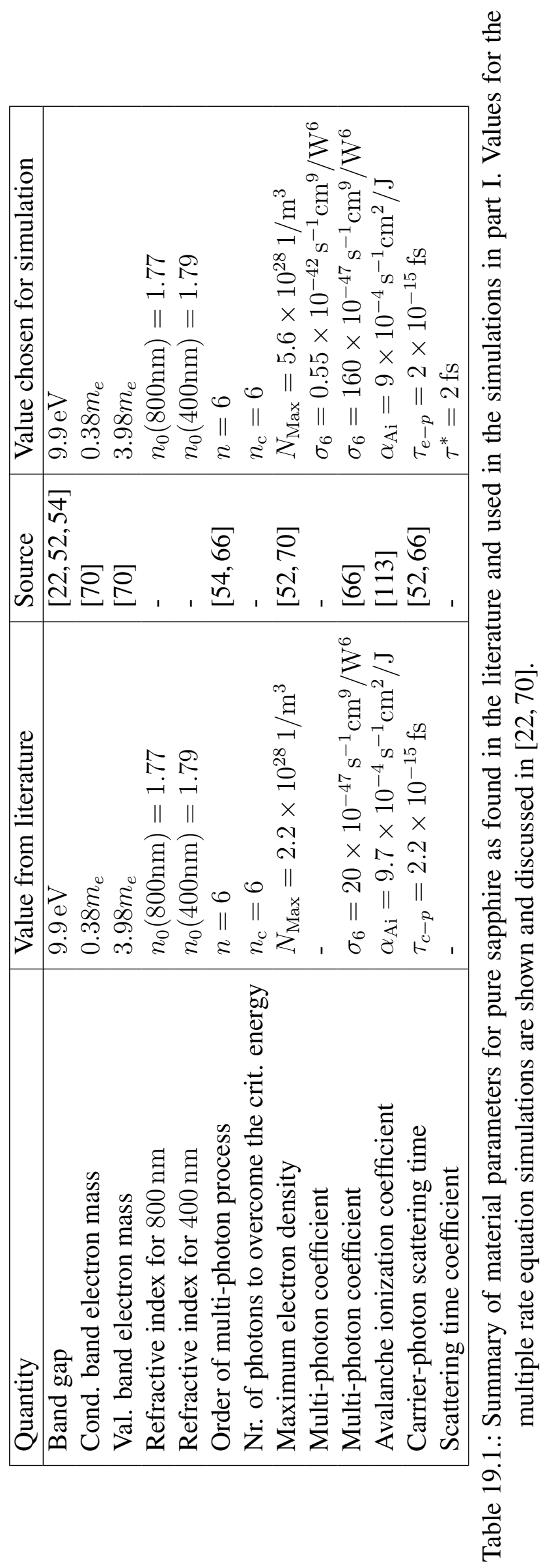




\section{Quantities for the calculation of laser excitation of water}

The maximum density of valence electrons of water has been estimated by the density of $\mathrm{H}_{2} \mathrm{O}$ molecules per cubic meter:

$$
\rho^{*}\left(\mathrm{H}_{2} \mathrm{O}\right)=N_{\mathrm{Av}} \times \frac{\rho_{\mathrm{H} 20}}{M}
$$

With the molecular mass of water $M\left(\mathrm{H}_{2} \mathrm{O}\right)=18$ and a density of $\rho=1 \mathrm{~g} / \mathrm{cm}^{3}$ we obtain $\rho^{*}=$ $3.3 \times 10^{22} \mathrm{H}_{2} \mathrm{O} / \mathrm{cm}^{3}$. With the one valence electron per hydrogen and the six from oxygen we end up with an approximated valence electron density of:

$$
N_{\text {Max }}=2.67 \times 10^{29} 1 / \mathrm{m}^{3}
$$




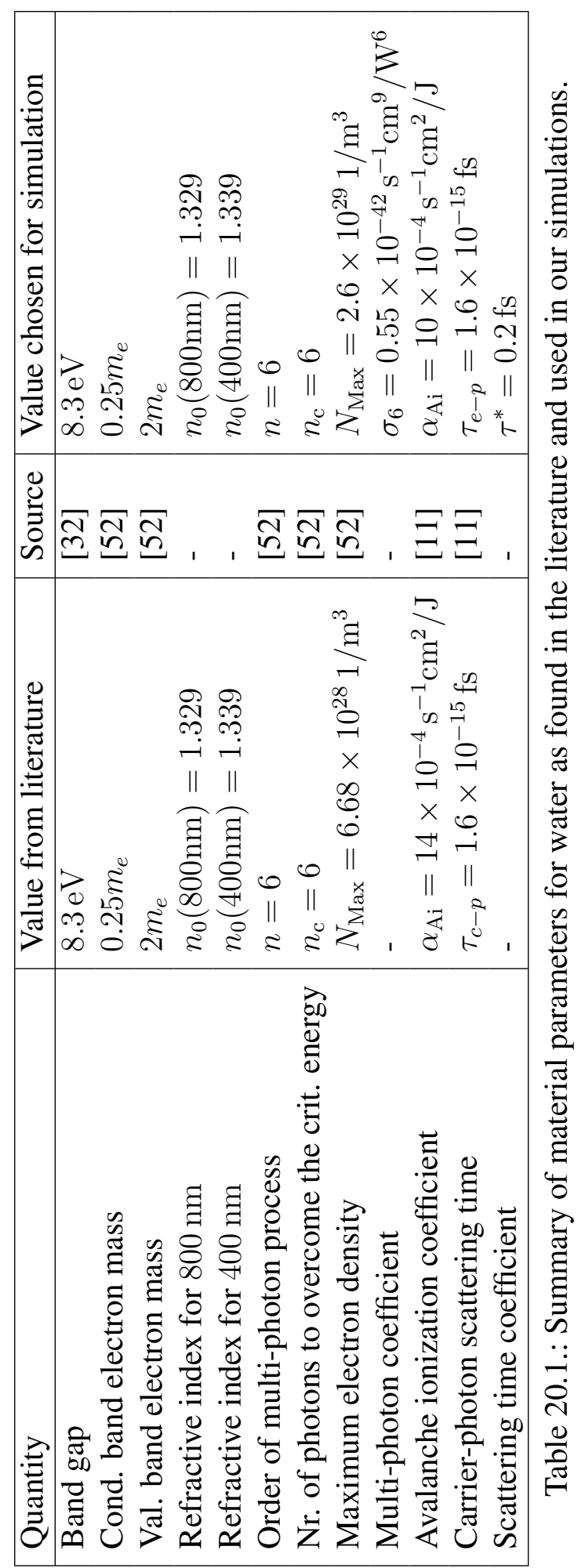




\section{Bibliography}

[1] Gattass, R. R.; MAZUR, E.: Femtosecond laser micromachining in transparent materials. In: Nature Photonics 2 (2008), S. 219-225. - ISSN 1749-4885

[2] Chung, Samuel H. ; MAzur, Eric: Surgical applications of femtosecond lasers. In: Journal of Biophotonics 2 (2009), Nr. 10, S. 557-572. http://dx.doi.org/10.1002/ jbio.200910053. - DOI 10.1002/jbio.200910053. - ISSN 1864-0648

[3] SHEN, Y. R.: Electrostriction, optical Kerr effect and self-focusing of laser beams. In: Physics Letters 20 (1966), Nr. 4, S. 378-380. http://dx.doi.org/10.1016/ 0031-9163(66) 90749-9. - DOI 10.1016/0031-9163(66)90749-9. - ISSN 00319163

[4] Islam, M. N. ; Simpson, J. R. ; Shang, H. T. ; Mollenauer, L. F. ; Stolen, R. H.: Cross-phase modulation in optical fibers. In: Optics Letters 12 (1987), Nr. 8, S. 625-627. http://dx.doi.org/10.1364/OL.12.000625, - DOI 10.1364/OL.12.000625. ISSN 0146-9592

[5] Schiffrin, Agustin ; PAasch-Colberg, Tim ; Karpowicz, Nicholas ; Apalkov, Vadym ; Gerster, Daniel ; Muhlbrandt, Sascha ; Korbman, Michael ; Reichert, Joachim ; Schultze, Martin ; Holzner, Simon ; BARTh, Johannes V. ; KienBerger, Reinhard ; ERnstorfer, Ralph ; YAKovlev, Vladislav S. ; Stockman, Mark I. ; KRAUSZ, Ferenc: Optical-field-induced current in dielectrics. In: Nature 493 (2013), Nr. 7430, S. 70-74. http://dx.doi.org/10.1038/nature11567, - DOI 10.1038/nature11567. - ISSN 0028-0836

[6] Szameit, Alexander ; Nolte, Stefan: Discrete optics in femtosecond-laser-written photonic structures. In: Journal of Physics B: Atomic, Molecular and Optical Physics 43 (2010), Nr. 16, S. 163001. http: //dx.doi.org/10.1088/0953-4075/43/16/163001. - DOI 10.1088/0953-4075/43/16/163001. - ISSN 0953-4075

[7] Mermillod-Blondin, A. ; Mauclair, C. ; Bonse, J. ; Stoian, R. ; Audouard, E. ; Rosenfeld, A. ; HeRTEl, I. V.: Time-resolved imaging of laser-induced refractive index changes in transparent media. In: Review of Scientific Instruments 82 (2011), S. 33703. ISSN 0034-6748

[8] Rapp, S. ; Heinrich, G. ; Wollgarten, M. ; Huber, H. P. ; Schmidt, M.: Physical mechanisms of SiNx layer structuring with ultrafast lasers by direct and confined laser ablation. In: Journal of Applied Physics 117 (2015), Nr. 10, S. 105304. http: //dx.doi.org/10.1063/1.4914457, - DOI 10.1063/1.4914457. - ISSN 00218979 
[9] Winkler, S. W. ; Burakov, I. M. ; Stoian, R. ; Bulgakova, N. M. ; Husakou, A. ; Mermillod-Blondin, A. ; Rosenfeld, A. ; Ashienasi, D. ; Hertel, I. V.: Transient response of dielectric materials exposed to ultrafast laser radiation. In: Applied Physics A: Materials Science and Processing 84 (2006), S. 413-422. - ISSN 0947-8396

[10] Winkler, Thomas ; Lillevang, Lasse-Haahr ; SARPe, Cristian ; Zielinski, Bastian ; Götte, Nadine ; Senftleben, Arne ; Balling, Peter ; Baumert, Thomas: Laser amplification in excited dielectrics. In: Nature Physics 14 (2018), S. 74-79. http: / / dx . doi.org/10.1038/nphys 4265. - DOI 10.1038/nphys4265

[11] SARPe, C. ; Köhler, J. ; Winkler, Thomas ; Wollenhaupt, M. ; B Aumert, Thomas: Real-time observation of transient electron density in water irradiated with tailored femtosecond laser pulses. In: New Journal of Physics 14 (2012), S. 75021. - ISSN 1367-2630

[12] Winkler, Thomas ; S ArPe, Cristian ; Jelzow, Nikolai ; Lillevang, Lasse H. ; Götte, Nadine ; Zielins Ki, Bastian ; BAlling, Peter ; SEnftleben, Arne ; BAuMert, Thomas: Probing spatial properties of electronic excitation in water after interaction with temporally shaped femtosecond laser pulses: Experiments and simulations. In: Applied Surface Science 374 (2016), S. 235-242. http://dx.doi.org/10.1016/j.apsusc.2015.11. 182. - DOI 10.1016/j.apsusc.2015.11.182. - ISSN 0169-4332

[13] Götte, Nadine ; Winkler, Thomas ; Meinl, Tamara ; Kusserow, Thomas ; ZielinSKI, Bastian ; SARPe, Cristian ; Senftleben, Arne ; Hillmer, Hartmut ; Baumert, Thomas: Temporal Airy pulses for controlled high aspect ratio nanomachining of dielectrics. In: Optica 3 (2016), Nr. 4, S. 389. http://dx.doi.org/10.1364/ OP TICA.3.000389. - DOI 10.1364/OPTICA.3.000389. - ISSN 2334-2536

[14] GöTte, Nadine: Optimierung von Nanostrukturen in Dielektrika fã $\frac{1}{4} r$ biomedizinische und technische Applikationen durch zeitlich geformte Femtosekunden-Laserpulse. Kassel, Universität Kassel, Dissertation, 2017

[15] Courvoisier, Sebastien ; Saklayen, Nabiha ; Huber, Marinus ; Chen, Jun ; Diebold, Eric D. ; Bonacina, Luigi ; Wolf, Jean-Pierre ; Mazur, Eric: Plasmonic Tipless Pyramid Arrays for Cell Poration. In: Nano Letters 15 (2015), Nr. 7, S. 4461-4466. http://dx.doi.org/10.1021/acs.nanolett.5b01697, - DOI 10.1021/acs.nanolett.5b01697. - ISSN 1530-6984

[16] TrÄGER, F. (Hrsg.): Springer Handbook of Lasers and Optics. Dordrecht Heidelberg London New York : Springer, 2012

[17] Brabec, T. ; Krausz, F.: Intense few-cycle laser fields: Frontiers of nonlinear optics. In: Reviews of Modern Physics 72 (2000), S. 545-591. - ISSN 0034-6861

[18] Köhler, J. ; Wollenhaupt, M. ; Bayer, T. ; S Arpe, C. ; Baumert, Thomas: Zeptosecond precision pulse shaping. In: Optics Express 19 (2011), S. 11638-11653. - ISSN 1094-4087

[19] Wollenhaupt, M. ; Assion, A. ; Baumert, Thomas: Femtosecond Laser Pulses: Linear Properties, Manipulation, Generation and Measurement. In: TRÄGER, F. (Hrsg.): Springer Handbook of Lasers and Optics. Springer Science and Business Media, 2007, S. 937-983 
[20] Wollenhaupt, M. ; Englert, L. ; Horn, A. ; Baumert, Thomas: Temporal femtosecond pulse tailoring for nanoscale laser processing of wide-bandgap materials. In: Proceedings of SPIE 7600 (2010), S. 76000X-76000X-11. - ISSN 0277-786X

[21] Ashcroft, Neil W. ; Mermin, N. D.: Solid state physics. College ed. Fort Worth : Saunders College Pub, 1976. - ISBN 978-0030839931

[22] Garcia-Lechuga, M. ; HaAhr-Lillevang, L. ; Siegel, J. ; B Alling, P. ; Guizard, S. ; SolIS, J.: Simultaneous time-space resolved reflectivity and interferometric measurements of dielectrics excited with femtosecond laser pulses. In: Physical Review B 95 (2017), Nr. 21, S. 214114. http://dx.doi.org/10.1103/PhysRevB.95.214114, DOI 10.1103/PhysRevB.95.214114. - ISSN 1098-0121

[23] Daguzan, Ph. ; Martin, P. ; Guizard, S. ; Petite, G.: Electron relaxation in the conduction band of wide-band-gap oxides. In: Physical Review B 52 (1995), S. 1709917105. - ISSN 1098-0121

[24] Guizard, S. ; Martin, P. ; Patite, G. ; D’Oliveira, P. ; Meynadier, P.: Timeresolved study of laser-induced colour centres in SiO2. In: Journal of Physics: Condensed Matter 8 (1996), S. 1281-1290. - ISSN 0953-8984

[25] Martin, P. ; Guizard, S. ; Daguzan, Ph. ; Petite, G. ; D’ Oliveira, P. ; Meynadier, P. ; PERDiX, M.: Subpicosecond study of carrier trapping dynamics in wide-band-gap crystals. In: Physical Review B 55 (1997), Nr. 9, S. 5799-5810. - ISSN 1098-0121

[26] Vogel, A.; Venugopalan, V.: Mechanisms of pulsed laser ablation of biological tissues. In: Chemical Reviews 103 (2003), Nr. 2, S. 577-644. - ISSN 0009-2665

[27] Vogel, A. ; Noack, J. ; Nahen, K. ; Theisen, D. ; Busch, S. ; Parlitz, U. ; Hammer, D. X. ; Noojin, G. D. ; Rockwell, B. A. ; Birngruber, R.: Energy balance of optical breakdown in water at nanosecond to femtosecond time scales. In: Applied Physics B: Lasers and Optics 68 (1999), Nr. 2, S. 271-280. - ISSN 0946-2171

[28] Vogel, A. ; Noack, J. ; Hüttman, G. ; Paltauf, G.: Mechanism of femtosecond laser nanosurgery of cells and tissues. In: Applied Physics B: Lasers and Optics 81 (2005), S. 1015-1047. - ISSN 0946-2171

[29] Vogel, A. ; Linz, N. ; Freidank, S. ; Paltauf, G.: Femtosecond-Laser-Induced Nanocavitation in Water: Implications for Optical Breakdown Threshold and Cell Surgery. In: Physical Review Letters 100 (2008), S. 038102-038102-4. - ISSN 0031-9007

[30] Courvoisier, S. ; Götte, N. ; Zielinski, B. ; Winkler, Thomas ; Sarpe, C. ; Senftleben, Arne ; Bonacina, L. ; Wolf, J. P. ; Baumert, Thomas: Temporal Airy pulses control cell poration. In: APL Photonics 1 (2016), Nr. 4, S. 46102. http://dx.doi.org/10.1063/1.4948367. - DOI 10.1063/1.4948367. - ISSN $2378-0967$

[31] LinZ, Norbert ; Freidank, Sebastian ; LiAng, Xiao-Xuan ; Vogel, Alfred: Wavelength dependence of femtosecond laser-induced breakdown in water and implications for laser surgery. In: Physical Review B 94 (2016), Nr. 2, S. 24113. http: / /dx . doi .org/10 . 1103/PhysRevB.94.024113. - DOI 10.1103/PhysRevB.94.024113. - ISSN 10980121 
[32] Elles, C. G. ; Jailaubekov, A. E. ; Crowell, R. A. ; Bradforth, S. E.: Excitationenergy dependence of the mechanism for two-photon ionization of liquid $\mathrm{H} 2 \mathrm{O}$ and D2O from 8.3 to 12.4 eV. In: The Journal of Chemical Physics 125 (2006), S. 044515-1-04451512. - ISSN 0021-9606

[33] Thomsen, C. L. ; Madsen, D. ; Keiding, S. R. ; Thøgersen, J. ; Christiansen, O.: Two-photon dissociation and ionization of liquid water studied by femtosecond transient absorption spectroscopy. In: The Journal of Chemical Physics 110 (1999), Nr. 7, S. 3453. http://dx.doi.org/10.1063/1.478212, - DOI 10.1063/1.478212. ISSN 0021-9606

[34] Keldysh, L. V.: Ionization in the field of a strong electromagnetic wave. In: Journal of Experimental and Theoretical Physics 20 (1965), S. 1307. - ISSN 1063-7761

[35] Balling, P. ; SCHOU, J.: Femtosecond-laser ablation dynamics of dielectrics: basics and applications for thin films. In: Reports on Progress in Physics 76 (2013), Nr. 3, 36502. ISI : 000315446800005 , - ISSN 0034-4885

[36] ReISs, H. R.: Complete Keldysh theory and its limiting cases. In: Physical Review A 42 (1990), Nr. 3, S. 1476-1486. - ISSN 1050-2947

[37] Born, M. ; Wolf, E.: Principles of optics -electromagnetic theory of propagation, interference and diffraction of light. Bd. 7th (exp.). Cambridge University Press, 1999. - ISBN $0-521-642221$

[38] Quéré, F. ; Guizard, S. ; Martin, P. ; Petite, G. ; Gobert, O. ; Meynadier, P. ; PERDRIX, M.: Ultrafast carrier dynamics in laser-excited materials: subpicosecond optical studies. In: Applied Physics B: Lasers and Optics 68 (1999), Nr. 3, S. 459-463. - ISSN 0946-2171

[39] Schaffer, C. B. ; Nishimura, N. ; Glezer, E. N. ; KIM, A. M.-T. ; MaZur, E.: Dynamics of femtosecond laser-induced breakdown in water from femtoseconds to microseconds. In: Optics Express 10 (2002), Nr. 3, S. 196-203. - ISSN 1094-4087

[40] Ridley, B. K.: Quantum Processes in Semiconductors. Bd. 4. Clarendon Press Oxford, 1999. - ISBN 0-19-850-580-9

[41] Bloembergen, N.: Laser-Induced Electric Breakdown in Solids. In: IEEE Journal of Quantum Electronics QE-10 (1974), Nr. 3, S. 375-386. - ISSN 0018-9197

[42] Feng, Q. ; Moloney, J. V. ; Newell, A. C. ; Wright, E. M. ; Cook, K. ; Kennedy, P. K. ; Hammer, D. X. ; Rockwell, B. A. ; Thompson, C. R.: Theory and Simulation on the Threshold of Water Breakdown Induced by Focused Ultrashort Laser Pulses. In: IEEE Journal of Quantum Electronics 33 (1997), Nr. 2, S. 127-137. - ISSN 0018-9197

[43] Gamaly, E. G. ; Rode, A. V.: Physics of ultra-short laser interaction with matter: From phonon excitation to ultimate transformations. In: Progress in Quantum Electronics 37 (2013), Nr. 5, 215-323. IS I : 000326999100001 , - ISSN 0079-6727

[44] HaAhr-Lillevang, L. ; W Ædegaard, K. ; Sandkamm, D. B. ; Mouskeftaras, A. ; Guizard, S. ; BALling, P.: Short-pulse laser excitation of quartz: Experiments and modelling of transient optical properties and ablation. In: Applied Physics A: Materials 
Science and Processing 120 (2015), Nr. 4, S. 1221-1227. http://dx.doi.org/10. 1007 /s00339-015-9307-9. - DOI 10.1007/s00339-015-9307-9. - ISSN 0947-8396

[45] Huthmacher, Klaus ; Molberg, Andreas K. ; Rethfeld, Bärbel ; Gulley, Jeremy R.: A split-step method to include electron-electron collisions via Monte Carlo in multiple rate equation simulations. In: Journal of Computational Physics 322 (2016), S. 535-546. http://dx.doi.org/10.1016/j.jcp.2016.06.043. - DOI 10.1016/j.jcp.2016.06.043. - ISSN 0021-9991

[46] RÄMER, Anika: Excitation and Relaxation Dynamics in Laser-Excited Semiconductors and Dielectrics, Technische Universität Kaiserslautern, Diss., 2017

[47] Chowdhury, I. H. ; Wu, A. Q. ; Xu, X. ; Weiner, A. M.: Ultra-fast laser absorption and ablation dynamics in wide-band-gap dielectrics. In: Applied Physics A: Materials Science and Processing 81 (2005), Nr. 8, S. 1627-1632. http://dx.doi.org/10.1007/ s00339-005-3326-x. - DOI 10.1007/s00339-005-3326-x. - ISSN 0947-8396

[48] Kharchenko, V. A. ; Rosen, M.: Auger relaxation processes in semiconductor nanocrystals and quantum wells. In: Journal of Luminescence 70 (1996), S. 158-169. - ISSN 0022-2313

[49] Miaja-Avila, L. ; Tritsch, J. R. ; Wolcott, A. ; Chan, W. L. ; Nelson, C. A. ; Zhu, X. Y.: Direct Mapping of Hot-Electron Relaxation and Multiplication Dynamics in PbSe Quantum Dots. In: Nano Letters 12 (2012), Nr. 3, 1588-1591. IS I : 000301406800081. - ISSN 1530-6984

[50] JACKSON, K.: Electric Fields in Electronic Structure Calculations: Electric Polarizabilities and IR and Raman Spectra from First Principles. In: DEÁK, P. (Hrsg.) ; FrAUENHEIM, T. (Hrsg.) ; Pederson, R. (Hrsg.): Computer Simulation of Materials at Atomic Level Bd. 1. Wiley, 2000. - ISBN 3-527-40290-X, S. 293-310

[51] Guizard, S. ; Semerok, A. ; Gaudin, J. ; Hashida, M. ; Martin, P. ; Quéré, F.: Femtosecond laser ablation of transparent dielectrics: measurement and modelisation of crater profiles. In: Applied Surface Science 186 (2002), S. 364-368. - ISSN 0169-4332

[52] Waedegaard, K. ; Frislev, M. ; Balling, P.: Femtosecond laser excitation of dielectric materials: experiments and modeling of optical properties and ablation depths. In: Applied Physics A: Materials Science and Processing 110 (2013), Nr. 3, 601-605. ISI:000321489000013, - ISSN 0947-8396

[53] Couairon, A. ; Mysyrowicz, A.: Femtosecond filamentation in transparent media. In: Physics Reports 441 (2007), S. 47-189. - ISSN 0370-1573

[54] Temnov, Vasily V.: Ultrafast Laser-induced Phenomena in Solids Studied by Timeresolved Interferometry, Universität Duisburg-Essen, Dissertation, 2004

[55] Stuart, B. C. ; Feit, M. D. ; Rubenchik, A. M. ; Shore, B. W. ; Perry, M. D.: LaserInduced Damage in Dielectrics with Nanosecond to Subpicosecond Pulses. In: Physical Review Letters 74 (1995), Nr. 12, S. 2248-2251. - ISSN 0031-9007

[56] Stuart, B. C. ; Feit, M. D. ; Herman, S. ; Rubenchik, A. M. ; Shore, B. W. ; Perry, M. D.: Nanosecond-to-femtosecond laser-induced breakdown in dielectrics. In: Physical Review B 53 (1996), Nr. 4, S. 1749-1761. - ISSN 1098-0121 
[57] MaO, S. S. ; ZenG, X. ; MAO, X. ; Russo, R. E.: Laser-induced breakdown spectroscopy: flat surface vs. cavity structures. In: Journal of Analytical Atomic Spectrometry 19 (2004), S. 495-498. - ISSN 0267-9477

[58] Wu, A. Q. ; Chowdhury, I. H. ; Xu, X. F.: Femtosecond laser absorption in fused silica: Numerical and experimental investigation. In: Physical Review B 72 (2005), Nr. 8, 085128-085128-7. IS I : 000231564600053 , - ISSN 1098-0121

[59] Mishina, E. D. ; Sherstyuk, N. E. ; Vorotilov, K. A. ; Sigov, A. S. ; Barberi, R. ; Moret, M. P. ; Manders, F. ; SAnto, M. P. d. ; Larsen, P. K. ; Rasing, T.: Nonlinear optical and electrostatic force microscopy for ferroelectric polarization imaging. In: Applied Physics B: Lasers and Optics 74 (2002), Nr. 7-8, S. 783-788. - ISSN 0946-2171

[60] Schaffer, C. B. ; Brodeur, A. ; Garcia, J. F. ; Mazur, E.: Micromachining bulk glass by use of femtosecond laser pulses with nanojoule energy. In: Optics Letters 26 (2001), Nr. 2, S. 93-95. - ISSN 0146-9592

[61] Lenzner, M. ; Krüger, J. ; Sartania, S. ; Cheng, Z. ; Spielmann, Ch. ; Mourou, G. ; KauteK, W. ; Krausz, F.: Femtosecond Optical Breakdown in Dielectrics. In: Physical Review Letters 80 (1998), Nr. 18, S. 4076-4079. - ISSN 0031-9007

[62] Temnov, V. V. ; Sokolowski-Tinten, K. ; Zhou, P. ; Linde, D. von d.: Femtosecond time-resolved interferometric microscopy. In: Applied Physics A: Materials Science and Processing 78 (2004), Nr. 4, S. 483-489. - ISSN 0947-8396

[63] Kennedy, P. K.: A First-Order Model for Computation of Laser-Induced Breakdown Thresholds in Ocular and Aqueous Media: Part I - Theory. In: IEEE Journal of Quantum Electronics 31 (1995), Nr. 12, S. 2241-2249. - ISSN 0018-9197

[64] RETHFELD, B.: Unified model for the free-electron avalanche in laser-irradiated dielectrics. In: Physical Review Letters 92 (2004), Nr. 18, S. 187401-1-187401-4. - ISSN 0031-9007

[65] Christensen, B. H. ; Balling, P.: Modeling ultrashort-pulse laser ablation of dielectric materials. In: Physical Review B 79 (2009), Nr. 15, S. 155424. http://dx. doi.org/10.1103/PhysRevB.79.155424. - DOI 10.1103/PhysRevB.79.155424. - ISSN 1098-0121

[66] Temnov, V. V. ; Sokolowski-Tinten, K. ; Zhou, P. ; El-Khamhawy, A. ; Linde, D. von d.: Multiphoton Ionization in Dielectrics: Comparison of Circular and Linear Polarization. In: Physical Review Letters 97 (2006), S. 237403. - ISSN 0031-9007

[67] Du, D. ; Liu, X. ; Korn, G. ; Squier, J. ; Mourou, G.: Laser-induced breakdown by impact ionization in $\mathrm{SiO} 2$ with pulse widths from 7 ns to 150 fs. In: Applied Physics Letters 64 (1994), Nr. 23, S. 3071-3073. - ISSN 0003-6951

[68] Jiang, L. ; TSAi, H. L.: Prediction of crater shape in femtosecond laser ablation of dielectrics. In: Journal of Physics D: Applied Physics 37 (2004), S. 1492-1496. - ISSN 0022-3727

[69] SACCHI, C. A.: Laser-Induced Electric Breakdown in Water. In: Journal of the Optical Society of America B: Optical Physics 8 (1991), Nr. 2, 337-345. IS I : A1991EW9780 0016. - ISSN 0740-3224 
[70] W GuizARD, Stéphane ; BALling, Peter: Probing ultrashort-pulse laser excitation of sapphire: From the initial carrier creation to material ablation. In: Europhysics Letters 105 (2014), Nr. 4, 47001. http://dx.doi.org/10.1209/0295-5075/105/47001. - DOI 10.1209/0295-5075/105/47001. - ISSN 0295-5075

[71] Siegel, J. ; Solis, J.: Imaging of plasma dynamics for controlled micromachining. Version:2012. http://link.springer.com/book/10.1007\% 2F978-3-642-23366-1. In: Femtosecond Laser Micromachining. Springer, 2012. - ISBN 978-3-642-23365-4, 19-41

[72] WINKLER, Thomas: Controlling Ionization Mechanisms in high Bandgap Dielectrics via Tailored Femtosecond Laser Pulses, Universität Kassel, Bachelor Thesis, 2012

[73] WAEDEGAARD, K. J.: Ultrashort-Pulse Laser Modification of Materials: Understanding and Controlling the Optical Properties, Aarhus University, PhD-Thesis, 2013

[74] Winkler, Mark T. ; Sher, Meng-Ju ; Lin, Yu-Ting ; Smith, Matthew J. ; Zhang, Haifei ; GRADEČAK, Silvija ; MAZUR, Eric: Studying femtosecond-laser hyperdoping by controlling surface morphology. In: Journal of Applied Physics 111 (2012), Nr. 9, S. 93511. http://dx.doi.org/10.1063/1.4709752. - DOI 10.1063/1.4709752. ISSN 0021-8979

[75] Rethfeld, Baerbel ; Ivanov, Dmitriy S. ; Garcia, Martin E. ; Anisimov, Sergei I.: Modelling ultrafast laser ablation. In: Journal of Physics D: Applied Physics 50 (2017), Nr. 19, 193001. http://stacks.iop.org/0022-3727/50/i=19/a=193001

[76] Schultze, M. ; Ramasesha, K. ; Pemmeraju, C. D. ; Sato, S. A. ; Whitmore, D. ; Gandman, A. ; Prell, J. S. ; Borja, L. J. ; Prendergast, D. ; Yabana, K. ; Neumark, D. M. ; Leone, S. R.: Attosecond band-gap dynamics in silicon. In: Science 346 (2014), Nr. 6215, S. 1348-1352. - ISSN 0036-8075

[77] Wollenhaupt, M. ; Assion, A. ; Baumert, Thomas: in: Springer Handbook of Lasers and Optics. Bd. 2. Springer, 2012

[78] JELzow, Nikolai: Charakterisierung und Erweiterung eines Gleicher-Pfad spektralem Interferometers zur Messung optischer Eigenschaften mittels geformter FemtosekundenLaserpulse angeregtem Wasser. Kassel, Universität Kassel, Bachelor-Arbeit, 2015

[79] Trebino, R.: Frequency-Resolved Optical Gating: The Measurement of Ultrashort Laser Pulses. Norwell,Massachusetts : Kluwer Academic Publishers, 2000

[80] KRUER, W.L.: The physics of laser plasma interactions. Addison-Wesley Pub. Co. Inc.,Reading, MA, 1988

[81] Takeda, M. ; INA, H. ; Kobayashi, S.: Fourier-transform method of fringe-pattern analysis for computer-based topography and interfermometry. In: Journal of the Optical Society of America 72 (1982), Nr. 1, S. 156-160. - ISSN 0030-3941

[82] Tokunaga, E. ; Terasaki, A. ; Kobayashi, T.: Frequency-domain interferometer for femtosecond time-resolved phase spectroscopy. In: Optics Letters 17 (1992), Nr. 16, S. 1131-1133. - ISSN 0146-9592 
[83] Giordmaine, J. A.: Nonlinear Optcal Properties of Liquids. In: Physical Review 138 (1965), S. A1599-A1606. - ISSN 0031-899X

[84] Hernandez-Rueda, J. ; Siegel, J. ; Galvan-Sosa, M. ; de la Cruz,A. R. ; Garcia-LechugA, M. ; Solis, J.: Controlling ablation mechanisms in sapphire by tuning the temporal shape of femtosecond laser pulses. In: Journal of the Optical Society of America B: Optical Physics 32 (2015), Nr. 1, 150-156. IS I : 000346868700018 , ISSN 0740-3224

[85] Armstrong, J. A. ; Bloembergen, N. ; Ducuing, J. ; Pershan, P. S.: Interactions between Light Waves in a Nonlinear Dielectric. In: Physical Review 127 (1962), Nr. 6, S. 1918-1939. http://dx.doi.org/10.1103/PhysRev.127.1918, - DOI 10.1103/PhysRev.127.1918. - ISSN 0031-899X

[86] Brouwer, N. ; RethfELD, B.: Excitation and relaxation dynamics in dielectrics irradiated by an intense ultrashort laser pulse. In: Journal of the Optical Society of America B: Optical Physics 31 (2014), Nr. 11, C28-C35. http://dx.doi.org/10.1364/JOSAB. 31. 000C28, - DOI 10.1364/JOSAB.31.000C28. - ISSN 0740-3224

[87] LANIER, Thomas E. ; Gulley, Jeremy R.: Nonlinear space-time focusing and filamentation of annular femtosecond pulses in dielectrics. In: Journal of the Optical Society of America B 33 (2016), Nr. 2, S. 292. http://dx.doi.org/10.1364/JOSAB. 33 . 000292, - DOI 10.1364/JOSAB.33.000292. - ISSN 0740-3224

[88] Winkler, S. W. ; Burakov, I. M. ; Stoian, R. ; Bulgakova, N. M. ; Husakou, A. ; Mermillod-Blondin, A. ; Rosenfeld, A. ; Ashienasi, D. ; Hertel, I. V.: Transient response of dielectric materials exposed to ultrafast laser radiation. In: Applied Physics A: Materials Science and Processing 84 (2006), S. 413-422. - ISSN 0947-8396

[89] Bulgakova, N. M. ; Stoian, R. ; Rosenfeld, A.: Laser-induced modification of transparent crystals and glasses. In: Quantum Electronics 40 (2010), Nr. 11, 966-985. IS I : 000286588700004 , - ISSN 1063-7818

[90] Johannsen, J. C. ; Ulstrup, S. ; Cilento, F. ; Crepaldi, A. ; Zacchigna, M. ; Cacho, C. ; Turcu, I. C. E. ; Springate, E. ; Fromm, F. ; Raidel, C. ; Seyller, T. ; Parmigiani, F. ; Grioni, M. ; Hofmann, P.: Direct View of Hot Carrier Dynamics in Graphene. In: Physical Review Letters 111 (2013), Nr. 2, 27403. IS I: 000321752400044 - ISSN 0031-9007

[91] Ebnonnasir, Abbas ; Narayanan, Badri ; Kodambaka, Suneel ; Ciobanu, Cristian V.: Tunable MoS2 bandgap in MoS2-graphene heterostructures. In: Applied Physics Letters 105 (2014), Nr. 3, S. 31603. http: / dx.doi.org/10.1063/1.4891430. DOI 10.1063/1.4891430. - ISSN 0003-6951

[92] Sokolowski-Tinten, K. ; Linde, D. von d.: Generation of dense electron-hole plasmas in silicon. In: Phys. Rev. B 61 (2000), Jan, 2643-2650. http://dx.doi.org/10. 1103/PhysRevB.61.2643. - DOI 10.1103/PhysRevB.61.2643

[93] Hernandez-Rueda, Javier ; Götte, Nadine ; Siegel, Jan ; Soccio, Michelina ; Zielinski, Bastian; SARPe, Cristian ; Wollenhaupt, Matthias ; EzQUerra, Tiberio A. ; BAUMERT, Thomas ; SOLIS, Javier: Nanofabrication of tailored surface structures in 
dielectrics using temporally shaped femtosecond-laser pulses. In: ACS Applied Materials \& Interfaces 7 (2015), Nr. 12, 6613-6619. http://dx.doi.org/10.1021/ am508925m, - DOI 10.1021/am508925m. - ISSN 1944-8252

[94] Sorokin, P. P. ; Braslau, N.: Some Theoretical Aspects of a Proposed Double Quantum Stimulated Emission Device. In: IBM Journal of Research and Development 8 (1964), April, Nr. 2, S. 177-181. http://dx.doi.org/10.1147/rd.82.0177, - DOI 10.1147/rd.82.0177. - ISSN 0018-8646

[95] Hayat, Alex ; Nevet, Amir ; Ginzburg, Pavel ; Orenstein, Meir: Applications of two-photon processes in semiconductor photonic devices: invited review. In: Semiconductor Science and Technology 26 (2011), Nr. 8, 083001. http://stacks.iop.org/ $0268-1242 / 26 / i=8 / a=083001$

[96] Lambropoulos, P.: Quantum Statistics of a Two-Photon Quantum Amplifier. In: Phys. Rev. 156 (1967), Apr, 286-297. http://dx.doi.org/10.1103/PhysRev.156. 286. - DOI 10.1103/PhysRev.156.286

[97] Yatsiv, Shaul ; Rokni, M. ; BARAK, S.: Enhanced Two-Proton Emission. In: Physical Review Letters 20 (1968), Nr. 23, S. 1282-1284. http://dx.doi.org/10.1103/ PhysRevLett.20.1282, - DOI 10.1103/PhysRevLett.20.1282. - ISSN 0031-9007

[98] Loy, M. M. T.: Two-Photon Adiabatic Inversion. In: Phys. Rev. Lett. 41 (1978), Aug, 473-476. http://dx.doi.org/10.1103/PhysRevLett.41.473. - DOI 10.1103/PhysRevLett.41.473

[99] Gauthier ; Wu ; Morin ; Mossberg: Realization of a continuous-wave, two-photon optical laser. In: Physical Review Letters 68 (1992), Nr. 4, S. 464-467. http://dx. doi.org/10.1103/PhysRevLett.68.464. - DOI 10.1103/PhysRevLett.68.464. ISSN 0031-9007

[100] Pfister, Olivier ; Brown, William J. ; Stenner, Michael D. ; Gauthier, Daniel J.: Two-photon stimulated emission in laser-driven alkali-metal atoms using an orthogonal pump-probe geometry. In: Physical Review A 60 (1999), Nr. 6, S. R4249-R4252. http://dx.doi.org/10.1103/PhysRevA.60.R4249. - DOI 10.1103/PhysRevA.60.R4249. - ISSN 1050-2947

[101] Belfield, Kevin D. ; Bondar, Mykhailo V. ; Yanez, Ciceron O. ; Hernandez, Florencio E. ; PRZHONSKA, Olga V.: One- and two-photon stimulated emission depletion of a sulfonyl-containing fluorene derivative. In: The journal of physical chemistry. $B 113$ (2009), Nr. 20, S. 7101-7106. http://dx.doi.org/10.1021/jp902060m. - DOI 10.1021/jp902060m. - ISSN 1520-6106

[102] IRONSIDE, C. N.: Two-photon gain semiconductor amplifier. In: IEEE Journal of Quantum Electronics 28 (1992), Nr. 4, S. 842-847. http://dx.doi.org/10.1109/3. 135201. - DOI 10.1109/3.135201. - ISSN 00189197

[103] Nevet, Amir ; Hayat, Alex ; Orenstein, Meir: Measurement of optical two-photon gain in electrically pumped AlGaAs at room temperature. In: Physical review letters 104 (2010), Nr. 20, S. 207404. http://dx.doi.org/10.1103/PhysRevLett.104.207404. - DOI 10.1103/PhysRevLett.104.207404. - ISSN 1079-7114 
[104] Reichert, Matthew ; SMirl, Arthur L. ; SAlamo, Greg ; Hagan, David J. ; van StrYLAND, Eric W.: Observation of Nondegenerate Two-Photon Gain in GaAs. In: Physical Review Letters 117 (2016), Nr. 7, S. 73602. http://dx.doi.org/10.1103/ PhysRevLett.117.073602. - DOI 10.1103/PhysRevLett.117.073602. - ISSN 00319007

[105] Elles, C. G. ; Shkrob, I. A. ; Crowell, R. A. ; Bradforth, S. E.: Excited state dynamics of liquid water: Insight from the dissociation reaction following two-photon excitation. In: The Journal of Chemical Physics 126 (2007), Nr. 16, S. 164503-164503-8. ISSN 0021-9606

[106] LinZ, Norbert ; Freidank, Sebastian ; Liang, Xiao-Xuan ; Vogelmann, Hannes ; TRICKL, Thomas ; VogEL, Alfred: Wavelength dependence of nanosecond infrared laserinduced breakdown in water: Evidence for multiphoton initiation via an intermediate state. In: Physical Review B 91 (2015), Nr. 13, S. 134114. http: / / dx.doi .org/10.1103/ PhysRevB.91.134114. - DOI 10.1103/PhysRevB.91.134114. - ISSN 1098-0121

[107] Wilkes, Z. W. ; Varma, S. ; Chen, Y.-H. ; Milchberg, H. M. ; Jones, T. G. ; Ting, A.: Direct measurements of the nonlinear index of refraction of water at 815 and $407 \mathrm{~nm}$ using single-shot supercontinuum spectral interferometry. In: Applied Physics Letters 94 (2009), Nr. 21, S. 211102. http://dx.doi.org/10.1063/1.3142384. - DOI 10.1063/1.3142384. - ISSN 0003-6951

[108] Bulgakova, N. M. ; Zhukov, V. P. ; Meshcheryakov, Y. P. ; Gemini, L. ; Brajer, J. ; Rostohar, D. ; MoceK, T.: Pulsed laser modification of transparent dielectrics: what can be foreseen and predicted by numerical simulations? In: Journal of the Optical Society of America B: Optical Physics 31 (2014), Nr. 11, C8-C14. IS I : 000344760800002 . ISSN 0740-3224

[109] Englert, L. ; Wollenhaupt, M. ; Rethfeld, B. ; HaAg, L. ; SARPe-Tudoran, C. ; BAUMERT, Thomas: Tailored Femtosecond Pulses for Nanoscale Laser Processing of Dielectrics. In: Proceeding of the Fourth International WLT-Conference on Lasers in Manufacturing 2007 (2007)

[110] Hernandez-Rueda, Javier ; Garcia-Lechuga, Mario ; Siegel, Jan ; Solis, Javier: Controlling ablation mechanisms in sapphire by irradiation with temporally shaped femtosecond laser pulses. In: Conferences on Lasers and Electro-Optics (2014), S. STh4B.3. http://dx.doi.org/10.1364/CLEO\{_\}SI.2014.STh4B.3, - DOI 10.1364/CLEO_SI.2014.STh4B.3

[111] Trebino, R. ; Delong, K. W. ; Fittinghoff, D. N. ; Sweetser, J. N. ; Krumbügel, M. A. ; Richman, B. A. ; KAne, D. J.: Measuring ultrashort laser pulses in the timefrequency domain using frequency-resolved optical gating. In: Review of Scientific Instruments 68 (1997), Nr. 9, S. 3277-3295. - ISSN 0034-6748

[112] Chandezon, J. ; Rampnoux, J-M ; Dilhaire, S. ; Audoin, B. ; Guillet, Y.: In-line femtosecond common-path interferometer in reflection mode. In: Optics Express 23 (2015), Nr. 21, S. 27011-27019. http://dx.doi.org/10.1364/OE.23.027011, - DOI 10.1364/OE.23.027011. - ISSN 1094-4087 
[113] Jasapara, J. ; RUdOlPh, W.: Characterization of sub-10-fs pulse focusing with highnumerical-aperture microscope objectives. In: Optics Letters 24 (1999), Nr. 11, S. 777-779. http://dx.doi.org/10.1364/OL.24.000777, - DOI 10.1364/OL.24.000777. ISSN 0146-9592 